\title{
Spectrum of cosmological correlation from vacuum fluctuation of stringy axion in entangled de Sitter space
}

\author{
Sayantan Choudhury ${ }^{1, a}{ }^{1 D}$, Sudhakar Panda ${ }^{2,3, b}$ \\ Am Mühlenberg 1, Golm, 14476 Potsdam, Germany \\ ${ }^{2}$ National Institute of Science Education and Research, Jatni, Bhubaneswar, Odisha 752050, India \\ ${ }^{3}$ Homi Bhabha National Institute, Training School Complex, Anushakti Nagar, Mumbai 400085, India
}

${ }^{1}$ Quantum Gravity and Unified Theory and Theoretical Cosmology Group, Max Planck Institute for Gravitational Physi (Albert Einstein Institute),

Received: 16 October 2019 / Accepted: 21 December 2019 / Published online: 28 January 2020

(C) The Author(s) 2020

\begin{abstract}
In this work, we study the impact of quantum entanglement on the two-point correlation function and the associated primordial power spectrum of mean square vacuum fluctuation in a bipartite quantum field theoretic system. The field theory that we consider is the effective theorry of axion field arising from Type IIB string theory compactified to four dimensions. We compute the expression for the powe spectrum of vacuum fluctuation in three different approach namely (1) field operator expansion (FOE) technique y/the the quantum entangled state, (2) reduced density matri (R) formalism with mixed quantum state and (3) th. me. $\mathrm{T}$ of non-entangled state (NES). For massless ax field, ih all these three formalism, we reproduce, at the eadi order, the exact scale invariant power spectrum which is well nown in the literature. We observe that due to c antum entanglement, the sub-leading terms for these thee for lism are different. Thus, such correction terms bre? analysis of the FOE, RDM and NES, alisms in the superhorizon limit. On the oth and, ior massive axion field we get a slight deviation $\mathrm{f}, \mathrm{m}$ sc le invariance and exactly quantify the spectral til of $\mathrm{t}$. nower spectrum in small scales. Apart from that massly s and massive axion field, we find distinguis ablo atures of the power spectrum for the FOE, RDY, and NES , $n$ the large scales, which is the result of quan $\ldots$ ang ement. We also find that such large-scale effects are na able to or greater than the curvature radius of the e Sitter space. Most importantly, in near future if $\mathrm{ex}_{\mathrm{H}}$ probe for early universe phenomena, one can detec ch small quantum effects. In such a scenario, it is possible to test the implications of quantum entanglement in primordial cosmology.
\end{abstract}

\footnotetext{
a e-mails: sayantan.choudhury @ aei.mpg.de; sayanphysicsisi@gmail.com

be-mail: panda@niser.ac.in
}

\section{Contents}

1 Introduction $\quad \ldots \ldots \ldots \ldots . \ldots \ldots$

2 War tion of axion in open chart ...... 4

2.1 ackground geometry . . . . . . . . . . 4

$2.2 \mathrm{~W}$ ave function for axion using Bunch Davies vacuum .............. 5

2.3 Wave function for axion using $\alpha$ vacua . . . . 7

Cosmological spectrum of quantum vacuum

fluctuation ................ 8

3.1 Quantum vacuum fluctuation using field operator expansion (FOE) (with entangled state) . . . 9

3.1.1 Wave function in field operator expansion (FOE) . . . . . . . . . . . 9

3.1.2 Two point correlation function . . . . . 10

3.2 Quantum vacuum fluctuation using reduced density matrix $(\mathrm{RDM})$ formalism (with mixed state) . . . . . . . . . . . . . . . . 14

3.2.1 Reduced density matrix (RDM) formalism 15

3.2.2 Two point correlation function . . . . . . 23

3.3 Quantum vacuum fluctuation with non entangled state (NES) . . . . . . . . . . . . 32

3.3.1 Non entangled state (NES) formalism . . 32

3.3.2 Two point correlation function . . . . . . 32

4 Summary ... . . . . . . . . . . . . 37

A Quantum correction to the power spectrum in FOE formalism . . . . . . . . . . . . . 40

A.1 For large wave number . . . . . . . . . . . . . . 41 4

A.2 For small wave number . . . . . . . . . . 43

B Quantum correction to the power spectrum in RDM formalism . . . . . . . . . . . . . 44

B.1 For large wave number . . . . . . . . . . . . 44

B.2 For small wave number . . . . . . . . . . . 46

C Quantum correction to the power spectrum in NES formalism . . . . . . . . . . . . . 48 
C.1 For large wave number . . . . . . . . . . . . . 48

C.2 For small wave number . . . . . . . . . . . . 49

References . . . . . . . . . . . . . . . . . . . 49

\section{Introduction}

The concept of quantum entanglement is one of the most interesting features that one can study in the context of quantum mechanics. Using such idea one can study the instantaneous physical implication of local measurements [1-3]. There are several applications in the framework of quantum field theory in which the quantum entanglement play a significant role. For example, particle creation (EPR Bell pair [4]) through the bubble nucleation procedure has been explained using the idea of quantum entanglement where the quantum system is strongly correlated [5-9]. Also using the concept of quantum entanglement in QFT one successfully explains many phenomena like entropy bounds, phase transitions, anomalies, confinement, thermalization and quantum critical quenches, localization in quantum gravity and description of interior of black holes. Apart from that quantum entanglement has huge application in the context of quantum information theory, quantum cryptography and interferometry.

The von- Neumann entropy and Rényi entropy are t' appropriate measures of quantum entanglement the frame work of condensed matter theory [10], in quantum iformation theory and in theoretical high energy physics. Th. doaf entanglement entropy in the context of quantu n field $t$ is the best possible computational tool to qran and stady the nature of the long range effects of av ntum ca lation. However, the computation of entangle ient entropy for a specific class of quantum field theories w not easy before the method proposed by Ryu and Takayana 1.1 . In this work, the authors have computed the rlement entropy for a strongly coupled field theory set un with a gravity dual using the techniques of holog ap and the results are remarkable as it is in agreement $\mathrm{WL}$ xpectations from the quantum field theory s'de [12-

Following th is - cess, Maldacena and Pimentel in Ref. [18] further prop sed an $\mathrm{c}$. 'cit technique to compute the entanglement ntropy in the framework of quantum field theory of do tter pace with Bunch Davies quantum initial $\ldots$ um $s$. Here, the authors have studied the gravi. on 1 of the quantum field theory of de Sitter spa "sing holographic techniques in detail. Further in Ref. [1) the authors have extended this computation in the context of $\alpha$ vacua [20-23] in the same context. In Refs. [24] and [25] the computation of quantum entanglement entropy and the formation of EPR Bell pair from stringy Axion were discussed with Bunch Davies and $\alpha$ vacua respectively.

Based on the physical set up used in our previous works [24] and [25], in this paper we have studied the cosmological implications of quantum entanglement by focussing on the long range effects of the two point correlation function computed from the mean square vacuum fluctuation of stringy Axion field with Bunch Davies and $\alpha$ quantum states as initial choice of vacua. We expect from this analysis that the signature and impact of quantum entanglement could be manifest in the correlation function even beyond the /ubble horizon scale. Our expectation is mainly due to the at do Sitter expansion of universe distinguish between a $\mathrm{P}$ of Axions [26-29], known as EPR Bell pair / hich is created within causally connected Hubble region Fo h is pose, we use three different techniques:

1. Field operator expan on $($ a) nethod with entangled state,

2. Reduced densit matrix to malism (RDM) with mixed state and

3. Non-enta $\%$ state $(, E S)$ method.

Here one can $a_{\text {a }}$ the following sets of questions regarding the imple ntation y 1 three different techniques in the present context

Q1. Why we have used three different formalisms to compute the cosmological two point correlation function? Q2. What is the correct physics they believe that happens in the setup of the space time?

- Q3. In those three formalisms, the physics is completely different. So which one is correct?

- Q4. We finally could only observe one possible observational consequence. So which one is correct?

The appropriate answers to above mentioned questions are appended below point wise:

- A1: We have used three different formalisms to compute the cosmological two point correlation function to check the explicit role of quantum mechanical entanglement in the primordial cosmology. In these three formalisms the leading order expressions become same. But the difference only can be found once we look into the small quantum corrections appearing in these formalisms. If the signature of quantum entanglement will be detected in near future in the observational probes of early universe, then one can explicitly rule out the possibility of appearing of NES method in the context of quantum field theory of primordial cosmology. On the other hand, if the signatures of quantum entanglement cannot be confirmed then one can strongly rely on the result obtained in the NES method. Additionally it is important to note that, these three frameworks provide us the quantum mechanical origin of quantum field theory of early universe cosmology. 
- A2 and A3: From the theoretical perspective these three different formalisms have their own merit on the physical ground. If the quantum mechanical origin of the quantum correction of the primordial fluctuation is coming from the non entangled state then NES formalism is the only single option which can take care of the correct physics. On the other hand, if the quantum mechanical origin of the quantum correction of the primordial fluctuation is coming from the entangled mixed state then RDM formalism applicable to the subsystem is the most promising option which supports correct physical explanation. The last option is FOE formalism which is applicable when the quantum mechanical origin of the quantum correction of the primordial fluctuation is guided by the total entangled state (not the subsystem) then FOE formalism is useful to describe the correct physics.

- A4: It is very well known fact that at late time scale all the large scale structure is formed due to long range persistent correlation originated from the primordial quantum mechanical fluctuation in the early universe. This can only be consistently theoretically established by using FOE and RDM formalisms which supports the concept of quantum entanglement in early universe cosmology. Now RDM formalism is more theoretically consistent than the FOE method as it is based on the quantum description if the reduced subsystem. Now as far as the detection in th observation is concerned, if we can detect the gaantum mechanical origin of the sub leading quantum tion in near future probes then one can explicitly the explicit role of quantum entangleme it, scisely rest FOE or RDM formalism is correct. N we cah tetect the role of quantum entanglemen then NES formalism will provide the correct physical e lanation of the quantum origin of the sub leading corre erm in the two point primordial correlation

We implem n $\mathrm{RDN}$ formalism using the previous work done by Malc na and Pimentel in Ref. [18] in the conte $\mathrm{t}$ of de Sitter cosmology. In our computation we have exph $\mathrm{V}$ inc uded the effect of Stringy Axion in the smin ld re $\mathrm{re}_{5}$ and as a result we get perturbatively cor$n$ ed cuinations in the expression for the power spectrum dern "using FOE, RDM and NES formalisms. Such correction ter ns can be interpreted as quantum effects which are appearing from the UV complete theory, such as a specific type of bipartite quantum field theory driven by axion. We note that the axion field which is being considered here, is actually originating from Type IIB string theory compactified on a Calabi-Yau three fold $\left(\mathbf{C Y}^{\mathbf{3}}\right)$, in presence of a NS5 brane sitting at the bottom of a long throat [30-33]. Most importantly, in the large wave number ${ }^{1}$ limit (small scale or small wave length approximation [34]) we have shown the results for the power spectrum derived from these three formalism perfectly match with each other if we consider only the leading order contribution. However, the results are different for these three formalisms if we we include the contributions from next and next to next leading order. In a way one can say that such additional small pe. b ive correction terms play a pivotal role to distinguish bet $n$ the FOE, RDM and NES formalisms. This is /bviously al/ important information because using the presen serv ional data on early universe cosmology [35-61] one ca a urther constrain the present model and also te the app opriateness of these formalisms. Apart from ${ }^{\prime}$ 's, fo $m r$ reteness, we have also analysed the behavio' of power spectrum in the small wave number limit rge scalo or large wave length approximation). We fina that "these three formalisms yield distinctive resul torms y the momentum (quantum number) depen nce $\varepsilon_{\text {the }}$ power spectrum in order by order. But the lack bservational data on this particular regime does n allow us o test the appropriateness and correctness of the prop a methods. We hope that in near future when the obse vational data for this regime will be available, our results can further constrain the model and rule out two of possibilities between the three formalisms discussed here. W would like to mention here that in our computation of the ower spectrum for mean square vacuum fluctuation we have not considered the quantum fluctuation of the pseudo scalar Axion field as a classical back ground field, the approach which is mostly used in the context of the cosmological correlations from early universe. Instead, we have chosen the field operator of the Axion field itself as quantum operator whose fluctuation with respect to a quantum mechanical vacuum state (Bunch Davies and $\alpha$ vacua). Thus, in this paper, we have followed:

1. A complete quantum approach to compute the primordial power spectrum of mean square vacuum fluctuation, which is not usually followed in the context of cosmology.

2. For the specific structure of the axion effective potential, we have computed the explicit form of the corrections which are due to quantum effects.

3. For our calculation, we have used three different approaches at super horizon time scale hoping that the quantum corrections, at small and large wave number limits when confronted with observations, can select the

\footnotetext{
${ }^{1}$ Here the wave number $p$ mimics the role of $\mathbf{S O}(\mathbf{3}, \mathbf{1})$ principal quantum number in the de Sitter hyperbolic open chart which is continuous and lying within the range $0<p<\infty$. The other $\mathbf{S O}(\mathbf{3}, \mathbf{1})$ quantum numbers $m$ (azimuthal) and $l$ (orbital) play no significant role in the final result as the expression for the power spectrum for mean square vacuum fluctuation only depends on the quantum number $p$.
} 




Fig. 1 Schematic diagram for the computation algorithm of long range effect of cosme ogica correlation function from quantum entanglement of axion in de Sitter open hyperbolic chart

most effective approach and the nature of quantum corrections. From the cosmological perspective we believe this is a very important step forward.

The plan of the paper is as follows: In Sect. 2, we begi our discussion with the computation of the wave furction of the Axion field in a de Sitter hyperbolic open chart. this purpose we have discussed the details of the backgrou.

Sitter geometrical set up in Sect. 2.1. Furt'ie Sects. 2.2 and 2.3, we have solved the total wave + nction. Axion for Bunch Davies vacuum and genera ised $\alpha$-vacua respectively. Using these solutions we have rived the cosmological power spectrum of mean square qua . vacuum fluctuation in Sect. 3. In Sects. 3.1.1 a 12 we have discussed the quantum vacuum fluctuation ing ield operator expansion (FOE) formalism vith ntang od state for Axion. field. We have also derivea form of the wave function in this form ism. colution is used to derive the power spectru $\mathrm{b}$. omputing the two point quantum correlation fun tion fron wean square vacuum fluctuation. In Sects. 3.2 and 3.2.2 We have discussed the quantum vacuum fluctuation ang $\mathrm{r}$-duced density matrix (RDM) formalism usi 10 ixed for Axion field and we have derived the 6. Tici a of the reduced density matrix in the de Sitter hyp olic open chart. Further, this result is used to derive the poy,er spectrum by computing the two point quantum correlation function from mean square vacuum fluctuation in large and small wave number limits for both massless and massve Axion fields. In Sects. 3.3.1 and 3.3.2 we have studied the quantum vacuum fluctuation using non entangled state (NES) formalism for Axion field and have discussed the NES formalism in detail. This result has been used to derive the pover $\rho_{\mathrm{r}}$ ctrum by computing the two point quantum correlation function from mean square vacuum fluctuation. Finally, Sect. 4 has been devoted to summery and conclusion I future prospects. In Fig. 1, we have presented a schematic di gram for the computation algorithm of long range effect of cosmological correlation function from quantum entanglement of axion in de Sitter open hyperbolic chart.

\section{Wave function of axion in open chart}

We briefly review here, for sake of completeness, the background geometry and the results for wave function of the axion field.

\subsection{Background geometry}

We consider a time preserving space-like hypersurface $\mathbf{S}^{\mathbf{2}}$ in the open hyperbolic chart of the de Sitter space. As a result $\mathbf{S}^{\mathbf{2}}$ is divided into two sub regions-interior and exterior which are identified by $\mathbf{R I}(\equiv \mathbf{L}) / \mathbf{R I I}(\equiv \mathbf{R})$. In terms of the Lorentzian signature an open chart in de Sitter space is described by three different subregions:

$$
\begin{aligned}
& \mathbf{R}(=\mathbf{R I I}) / \mathbf{L}(=\mathbf{R I}): \\
& \begin{cases}\tau_{\mathrm{E}}= \pm \frac{\pi}{2} \mp i t_{\mathbf{R} / \mathbf{L}} & t_{\mathbf{R}} \geq 0 / t_{\mathbf{L}} \geq 0 \\
\rho_{\mathrm{E}}=-i r_{\mathbf{R} / \mathbf{L}} & r_{\mathbf{R}} \geq 0 / r_{\mathbf{L}} \geq 0 \\
d s_{\mathbf{R} / \mathbf{L}}^{2}=\frac{1}{H^{2}}\left[-d t_{\mathbf{R} / \mathbf{L}}^{2}+\sinh ^{2} t_{\mathbf{R} / \mathbf{L}}\right. & (2.1) \\
\left.\times\left(d r_{\mathbf{R} / \mathbf{L}}^{2}+\sinh ^{2} r_{\mathbf{R} / \mathbf{L}} d \Omega_{2}^{2}\right)\right] & \end{cases}
\end{aligned}
$$



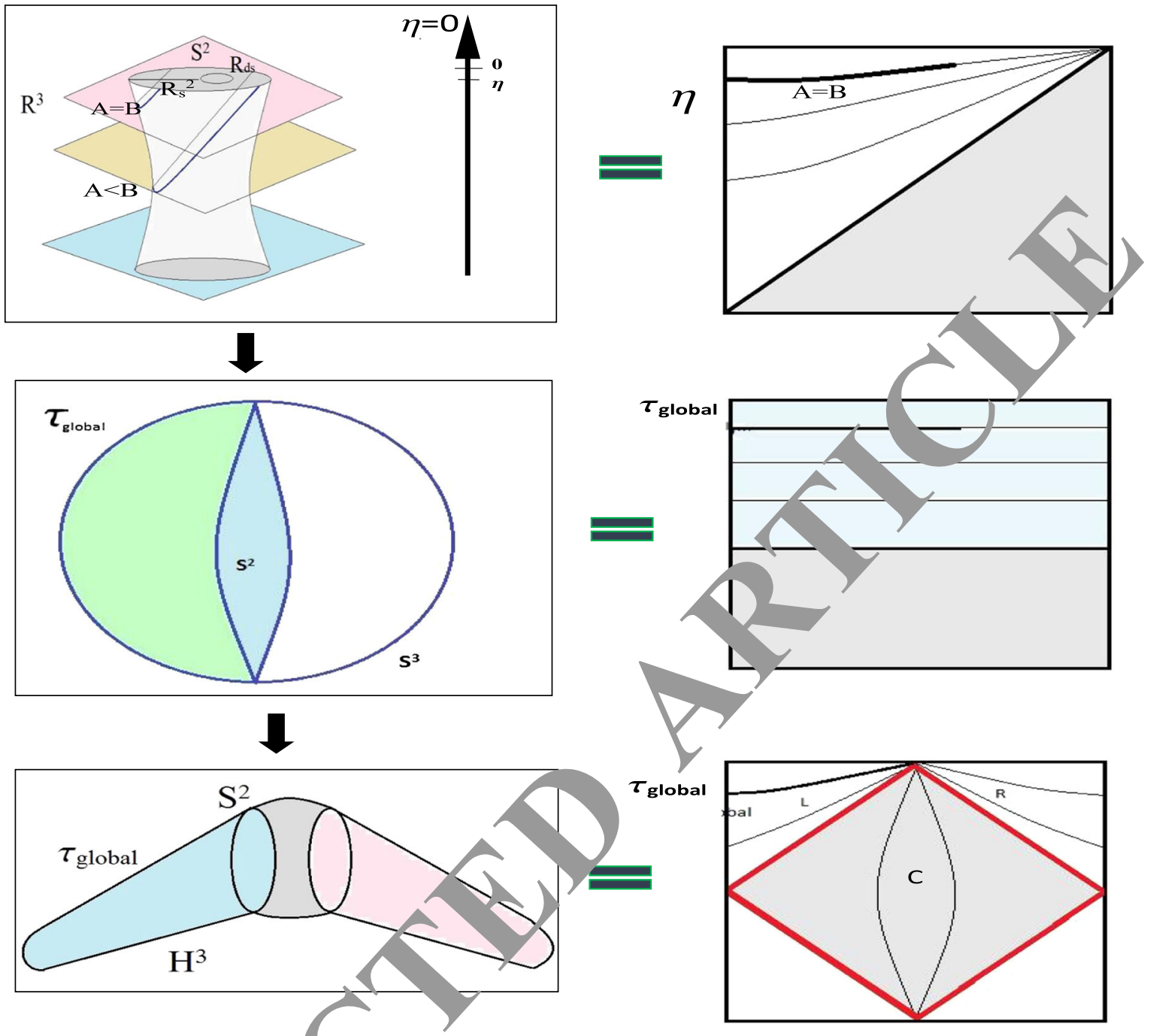

Fig. 2 Schematic diagram for the geometri constru tion and underlying symmetries of the bipartite quantum field theoretic system of de Sitter hyperbolic open chart. Corresponding Penros are also drawn for completeness



where $H=\dot{\sim} /$ is thu parameter and $d \Omega_{2}^{2}$ represents angular $\mathbf{n}$ irt of the metric on $\mathbf{S}^{2}$. Now let us assume that the total Hilbo bace ff the local quantum mechanical system is des $\rightarrow \mathrm{d}$ by, which can be written using bipartite decomitio direct product space as, $\mathcal{H}=\mathcal{H}_{\text {INT }} \otimes \mathcal{H}_{\text {EXT }}$. Her ' interior and exterior region and describe the localised modes in $\mathbf{R I} / \mathbf{R I I}$ respectively.

In Fig. 2 we have shown the schematic diagram for the geometrical construction and underlying symmetries of the bipartite quantum field theoretic system of de Sitter hyperbolic open chart. Corresponding Penrose diagrams are also drawn for completeness.

\subsection{Wave function for axion using Bunch Davies vacuum}

Though our prime objective is to compute the cosmological correlation functions for axion field in de Sitter space, we need the results for the wave function of the axion field in the just mentioned geometrical set up. Note that he axion field under consideration is coming from RR sector of Type IIB string theory compactified on $\mathbf{C Y}^{\mathbf{3}}$ in presence of NS 5 brane [30-33,69]. The effective action for the axion field is given by [30-33]:

$$
\begin{aligned}
S_{\text {axion }}= & \int d^{4} x \sqrt{-g}\left[-\frac{1}{2}(\partial \phi)^{2}\right. \\
& \left.+\mu^{3}\left\{\phi+b f_{a} \cos \left(\frac{\phi}{f_{a}}\right)\right\}\right],
\end{aligned}
$$


Fig. 3 Behaviour of the axion effective potential obtained from Type IIB String Theory with respect to the dimensionless field value $\phi / f_{a}$, where $f_{a}$ is the axion decay constant

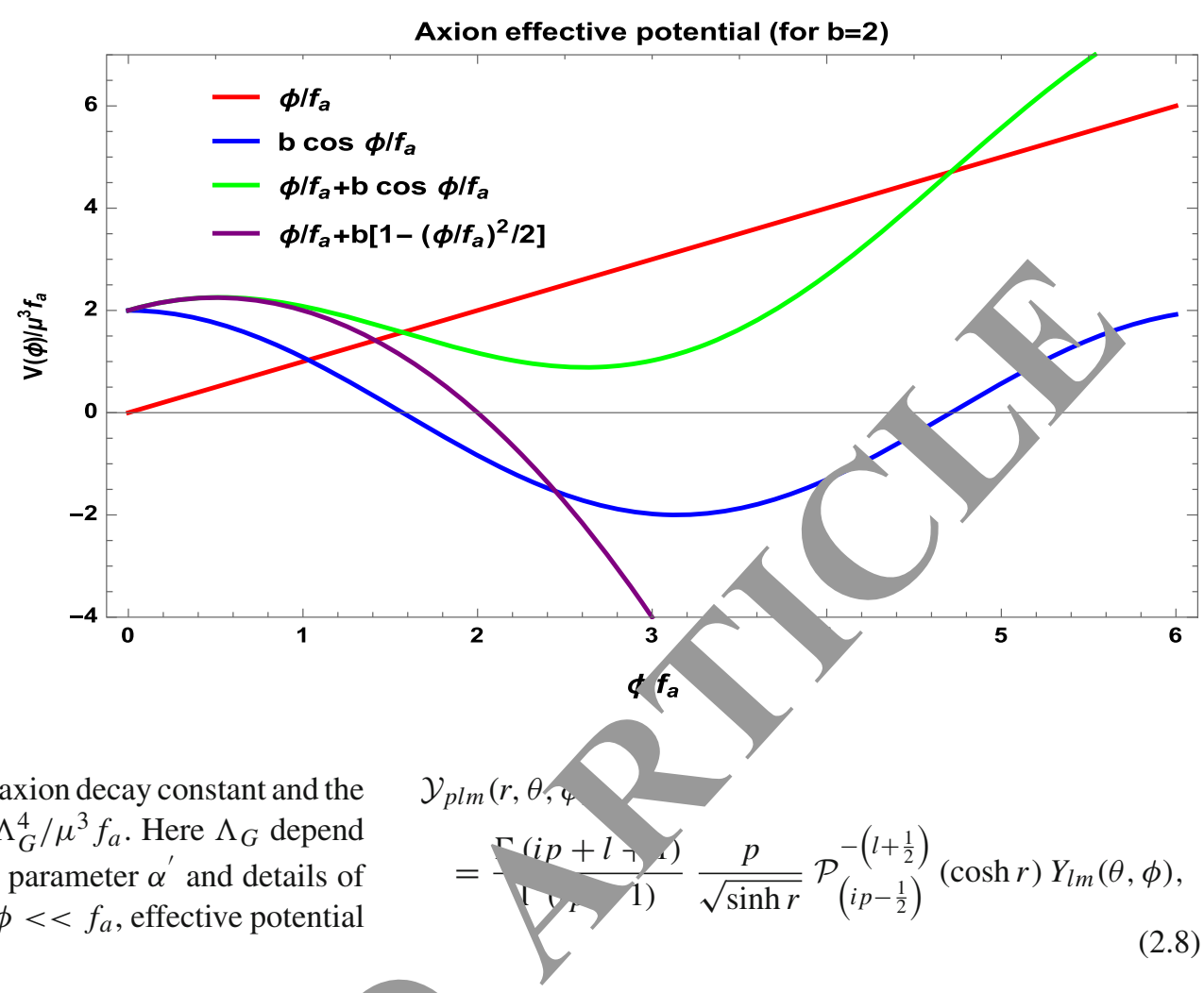

where $\mu^{3}$ is the mass scale, $f_{a}$ is axion decay constant and the parameter $b$ is defined as, $b=\Lambda_{G}^{4} / \mu^{3} f_{a}$. Here $\Lambda_{G}$ depend on the string coupling $g_{s}$, slope parameter $\alpha^{\prime}$ and details of SUSY breaking parameter. For $\phi<<f_{a}$, effective potential for axion can be expressed as:

$V(\phi) \approx \mu^{3}\left(b f_{a}+\phi\right)-\frac{m_{\text {axion }}^{2}}{2} \phi^{2}$

where we introduce the effective mass of the riol as, $m_{\text {axion }}^{2}=\mu^{3} b / f_{a}=\Lambda_{G}^{4} / f_{a}^{2}$. Here axion decay ons follow a (conformal) time dependent profile, wh is explic ay mentioned in references.

In Fig. 3 we have explicitly presenter. the behavic ar of the above axion potential with respect to e dimensionless field value $\phi / f_{a}$.

Further using Eq. (2.3) the fic ${ }^{1 / d}$ equation of motion for the axion can be written as:

$\left[\frac{1}{a^{3}(t)} \partial_{t}\left(a^{3}(t) \partial_{t}\right)-\widetilde{v^{2}}-\widetilde{\mathbf{L}^{2}} \mathbf{A}^{3}+m_{\text {axion }}^{2}\right] \phi=\mu^{3}$,

where the scalc fact $(t)$ in de Sitter open chart is given by, $a(t)=\operatorname{sir} n t y H$. Her the Laplacian operator $\widetilde{\mathbf{L}^{2}} \mathbf{H}^{3}$ in $\mathbf{H}^{3}$ can be $m$ tel as:

$\widetilde{\mathbf{L}^{2}}{ }^{3}=\frac{1}{{ }^{2}}\left[\partial_{r}\left(\sinh ^{2} r \partial_{r}\right)+\frac{1}{\sin \theta} \partial_{\theta}\left(\sin \theta \partial_{\theta}\right)+\frac{1}{\sin ^{2} \theta} \partial_{\phi}^{2}\right]$,

which satisfy the following eigenvalue equation:

$\widetilde{\mathbf{L}^{2}}{ }_{\mathbf{H}^{3}} \mathcal{Y}_{p l m}(r, \theta, \phi)=-\left(1+p^{2}\right) \mathcal{Y}_{p l m}(r, \theta, \phi)$.

Here $\mathcal{Y}_{\text {plm }}(r, \theta, \phi)$ represents orthonormal eigenfunctions which can be written in terms of a radial and angular part as: ere $Y_{l m}(\theta, \phi)$ is the spherical harmonics. Consequently, th total solution of the equations of motion can be written

$$
\begin{aligned}
\Phi(t, r, \theta, \phi)= & \sum_{\sigma= \pm 1} \sum_{Q=p, l, m} \\
& \times\left[a_{Q} \mathcal{V}_{Q}(t, r, \theta, \phi)+a_{Q}^{\dagger} \mathcal{V}_{Q}^{*}(t, r, \theta, \phi)\right]
\end{aligned}
$$

Here the total solution $\mathcal{V}_{Q}(t, r, \theta, \phi)$ for Bunch Davies vacuum can be expressed as:

$$
\begin{aligned}
\mathcal{V}_{Q}(t, r, \theta, \phi) & =\frac{1}{a(t)} \chi_{p, \sigma}(t) \mathcal{Y}_{p l m}(r, \theta, \phi) \\
& =\frac{H}{\sinh t} \chi_{p, \sigma}(t) \mathcal{Y}_{p l m}(r, \theta, \phi),
\end{aligned}
$$

where $\chi_{p, \sigma}(t)$ forms a complete set of positive frequency function. Also this can be written as a sum of complementary $\left(\chi_{p, \sigma}^{(c)}(t)\right)$ and particular integral $\left(\chi_{p, \sigma}^{(p)}(t)\right)$ part, as given by:

$\chi_{p, \sigma}(t)=\chi_{p, \sigma}^{(c)}(t)+\chi_{p, \sigma}^{(p)}(t)$.

Explicitly the solution for the complementary part and the particular integral part can be expressed as: 


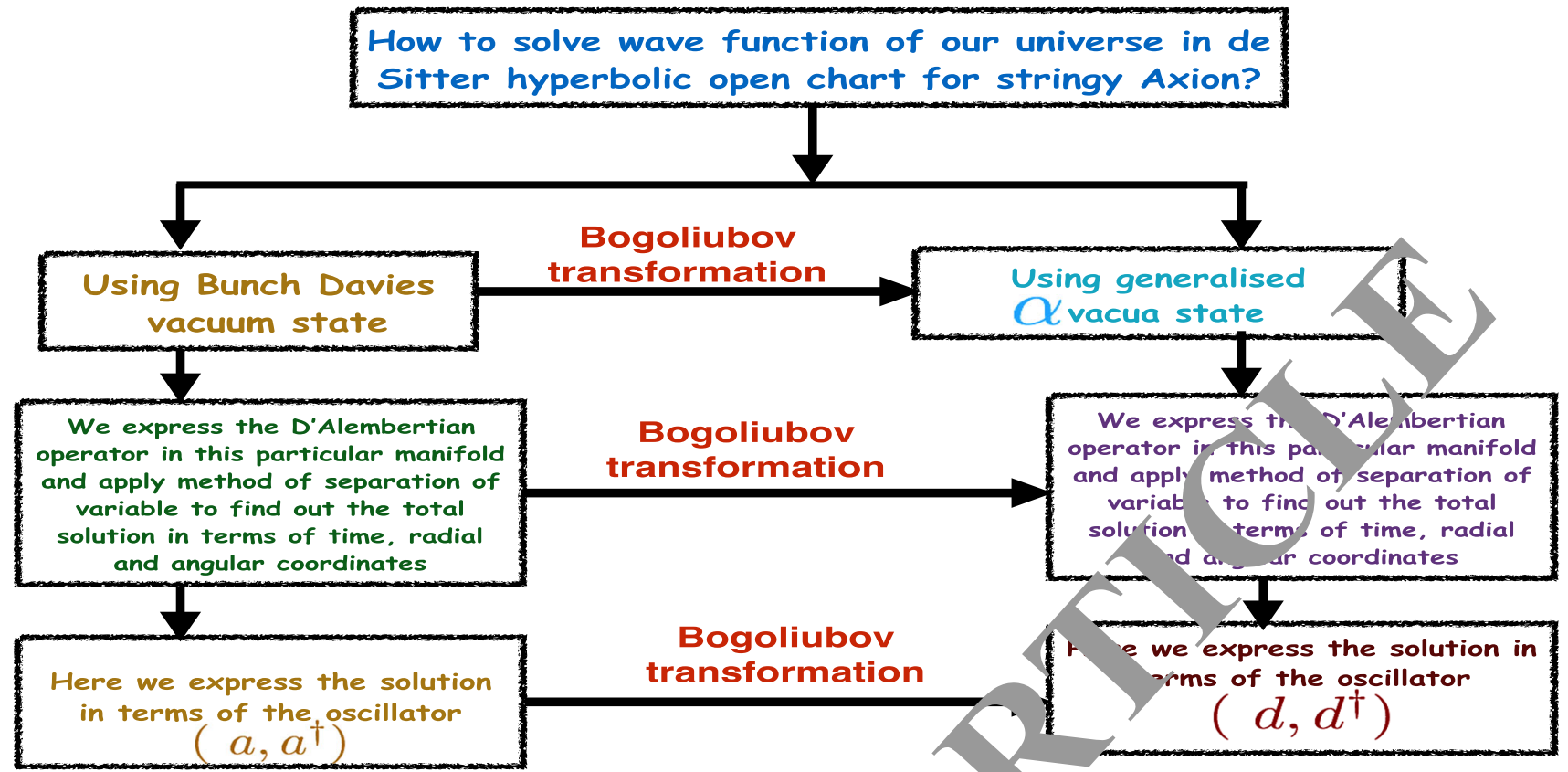

Fig. 4 Schematic diagram for the computation algorithm of solving the wave func of our un verse in de Sitter hyperbolic open chart for stringy axion

$\chi_{p, \sigma}^{(c)}(t)=\left\{\begin{array}{c}\frac{1}{2 \sinh \pi p}\left[\frac{\left(e^{\pi p}-i \sigma e^{-i \pi v}\right)}{\Gamma\left(v+\frac{1}{2}+i p\right)} \mathcal{P}_{\left(v-\frac{1}{2}\right)}^{i p}\left(\cosh t_{\mathbf{R}} \frac{(p-i \sigma)}{\Gamma(-i \pi v)} \mathcal{P}_{\left(v-\frac{1}{2}\right)}^{-i p}\left(\cosh t_{\mathbf{R}}\right)\right] \text { for } \mathbf{R}\right. \\ \frac{\sigma}{2 \sinh \pi p}\left[\frac{\left(e^{\pi p}-i \sigma e^{-i \pi v}\right)}{\Gamma\left(v+\frac{1}{2}+i p\right)} \mathcal{P}_{\left(v-\frac{1}{2}\right)}^{i p}\left(\cosh t_{\mathbf{L}}\right.\right. \\ \left.\frac{\left(e^{-\nu}-i \sigma e^{-i \pi v}\right)}{\Gamma\left(v+\frac{1}{2}-i p\right)} \mathcal{P}_{\left(v-\frac{1}{2}\right)}^{-i p}\left(\cosh t_{\mathbf{L}}\right)\right] \text { for } \mathbf{L},\end{array}\right.$

$\chi_{p, \sigma}^{(p)}(t)=\sinh ^{2} t \sum_{n=0}^{\infty} \frac{1}{\left(p^{2}-p_{n}^{2}\right)} \chi_{p_{n}, \sigma}^{(c)}(t) \jmath^{\prime} d t^{\prime} \chi_{p_{n}, \sigma}\left(t^{\prime}\right) \mu^{3}$.

where the parameter $v$ is defined as:

$v=\sqrt{\frac{9}{4}-\frac{m_{\text {axion }}^{2}}{H^{2}}}=\frac{9}{\frac{\mu^{3} b}{J_{a} H^{2}}}=\sqrt{\frac{9}{4}-\frac{\Lambda_{G}^{4}}{f_{a}^{2} H^{2}}}$.

In Fig. 4 ve have given a schematic diagram for the computation algu mof solving the wave function of our universe in $\mathrm{d}$ ter hy ibolic open chart for stringy axion.

\subsection{Wa ve function for axion using $\alpha$ vacua}

Here we use two subspaces in CPT invariant $\mathbf{S O}(\mathbf{1}, \mathbf{4})$ isometric de Sitter space, which is identified as RI and RII respectively. Use the result obtained for Bunch Davies vacuum, and performing a Bogoliubov transformation the mode functions for the $\alpha$-vacua can be expressed as:

$$
\begin{aligned}
& \Phi(r, t, \theta, \phi) \\
& =\int_{0}^{\infty} d p \sum_{\sigma= \pm 1} \sum_{l=0}^{\infty} \sum_{m=-l}^{+l} \\
& \quad \times\left[d_{\sigma p l m} \mathcal{F}_{\sigma p l m}^{(\alpha)}(r, t, \theta, \phi)+d_{\sigma p l m}^{\dagger}\left(\mathcal{F}_{\sigma p l m}^{(\alpha)}\right)^{*}(r, t, \theta, \phi)\right]
\end{aligned}
$$

where the $\alpha$-vacua state are defined as:

$$
\begin{aligned}
& d_{\sigma p l m}|\alpha\rangle=0 \quad \forall \sigma=(+1,-1) \\
& 0<p<\infty ; l=0, \ldots, \infty, m=-l, \ldots,+l .
\end{aligned}
$$

In this context, the $\alpha$-vacua mode function $\mathcal{F}_{\sigma p l m}^{(\alpha)}$ can be expressed in terms of Bunch Davies mode function $\mathcal{V}_{\sigma p l m}(r, t, \theta, \phi)$ using Bogoliubov transformation as:

$$
\begin{aligned}
& \mathcal{F}_{\sigma p l m}^{(\alpha)}=\left[\cosh \alpha \mathcal{V}_{\sigma p l m}(r, t, \theta, \phi)\right. \\
& \left.+\sinh \alpha \mathcal{V}_{\sigma p l m}^{*}(r, t, \theta, \phi)\right] .
\end{aligned}
$$


Here $\mathcal{V}_{\sigma p l m}(r, t, \theta, \phi)$ is the Bunch Davies vacuum states, which is defined as:

$\mathcal{V}_{\sigma p l m}(r, t, \theta, \phi)=\frac{H}{\sinh t} \chi_{p, \sigma}(t) \mathcal{Y}_{p l m}(r, \theta, \phi)$.

After substituting Eqs. (2.17) and (2.18) in Eq. (2.15) we get the following expression for the wave function:

$$
\begin{aligned}
\Phi(r, t, \theta, \phi)= & \frac{H}{\sinh t} \int_{0}^{\infty} d p \sum_{\sigma= \pm 1} \sum_{l=0}^{p-1} \sum_{m=-l}^{+l} \\
& \times\left[d_{\sigma p l m} \cosh \alpha \chi_{p, \sigma}(t)\right. \\
& \left.+d_{\sigma p l m}^{\dagger} \sinh \alpha \chi_{p, \sigma}^{*}(t)\right] \mathcal{Y}_{p l m}(r, \theta, \phi),
\end{aligned}
$$

Finally, the solution of the time dependent part of the wave function can be recast as:

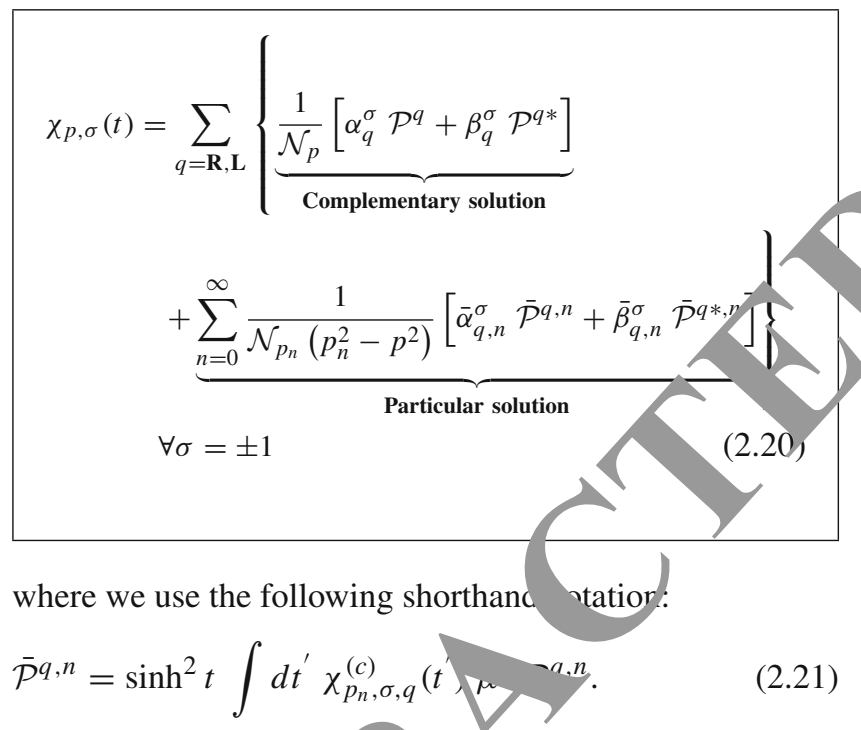

Here we also use the sho hand rotations $\mathcal{P}^{q}, \mathcal{P}^{q, n}$, for the Legendre polynor 1 the coefficient functions $\left(\alpha_{q}^{\sigma}, \beta_{q}^{\sigma}\right)$ and $\left(\alpha^{\sigma}, \beta_{q, n}^{\sigma}\right)$, malization constants $\mathcal{N}_{p}, \mathcal{N}_{p_{n}}$ for the compl mo ry and particular part of the solution which are d ined as:

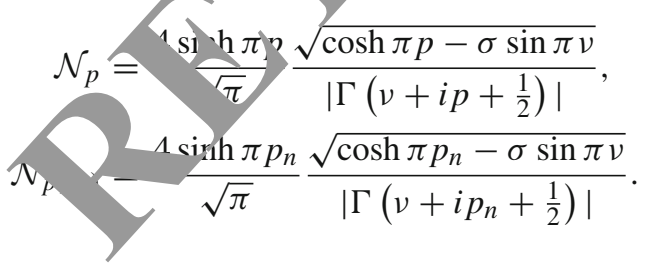

\section{Cosmological spectrum of quantum vacuum fluctuation}

In this section, we present our computation of the spectrum of Bunch Davies vacuum and $\alpha$ vacua fluctuation from two point correlation function. We will be discussing the computation of two point correlation function and their associated cosmological spectra from three completely different formalisms:-

\section{Field operator expansion (FOE) method:}

This method is useful for entangled quarcum states with the wave function of the de Sitter un rs for Bunch Davies and most generalised $\alpha$ vacua. To vically this formalism is based on the wave unction $\chi \frac{1}{}$ which we will explicitly derive. The rosh gica spectrum is characterised by the two r oint correr. on function and their associated power sp trum. Using such entangled state in this formalisp ne sonst the usual density matrix for Bunch Favic nd most generalised $\alpha$ vacua.

\section{Reduced densi matrix (OM) formalism:}

This formalis $n$ is "nful for mixed quantum states and is useful he cons uction of reduced density matrix in a di nal ropresentation of Bunch Davies and $\alpha$ vacua by $L_{\text {. }}$ ing over the all possible degrees of freedom fro the regiy $n \mathbf{R}$. Technically the formalism is based on the way nction $\psi^{\mathcal{I}}$ which we explicitly derive.

\section{Non entangled state (NES) formalism:}

This rormalism in presence of non entangled quantum state which deals with the construction of wave function in the region $\mathbf{L}$ in which the total universe is described. Here we also use Bunch Davies and most generalised $\alpha$ vacua in the region $\mathbf{L}$. Technically this formalism is based on the wave function $\phi^{\mathcal{I}}$ which we explicitly derive in this paper.

We will now derive the expression for the mean square fluctuation considering both Bunch Davies vacuum and $\alpha$ vacua using the results presented in the previous section. For this computation we will follow the steps which are outlined below:

1. First of all, we trace out all contributions which belong to the $\mathbf{R}$ region. As a result the required field operator is only defined in the $\mathbf{L}$ region. This method we use in FOE formalism where the quantum states for $\mathbf{L}$ and $\mathbf{R}$ region are entangled with each other. On the other hand, doing a partial trace over region $\mathbf{R}$ one can construct reduced density matrix which leads to RDM formalism. Instead, if we use the non entangled quantum state and compute the wave function solely in $\mathbf{L}$ region we will be lead to the NES formalism. Note that all of these three methods are used to compute mean square vacuum fluctuation or more precisely the quantum mechanical computation of two point correlation function for axion and the associated power spectrum.

2. Instead of doing the computation in $|\mathbf{L}\rangle$ basis we use a new basis $\left|\mathbf{L}^{\prime}\right\rangle$, obtained by applying Bogoliubov trans- 
formation in $|\mathbf{L}\rangle$. Consequently the field operators will act on $\left|\mathbf{L}^{\prime}\right\rangle$ and the FOE method is developed in this transformed basis. On the other hand, as mentioned earlier it will appear in the expression for the reduced density matrix to be used in the RDM formalism. But in the NES formalism this transformation is not very useful since in this case the total wave function is solely described by the quantum mechanical state appearing in the $\mathbf{L}$ region and the corresponding Hilbert space is spanned by only |L) which forms a complete basis.

3. Further, we will compute the expressions for the mean square quantum vacuum fluctuation and the corresponding cosmological power spectrum after horizon exit using all the three formalisms i.e. FOE, RDM and NES. We will finally consider two limiting situations: long wave length and short wave length approximation for the computation of the power spectrum.

\subsection{Quantum vacuum fluctuation using field operator} expansion (FOE) (with entangled state)

\subsubsection{Wave function in field operator expansion (FOE)}

Let us first compute the spectrum of vacuum fluctuation usin? field operator expansion (FOE). In Fig. 5 we have preser. a schematic diagram for the computation algorithm os field operator expansion method for entangled state of a on in de Sitter hyperbolic open chart. To compute the y cuu. fuctuation using FOE, we focus only with the region as it is completely symmetric to the right regin $\mathbf{k}$. We use the time dependent mode function for the lf it region $\mathbf{L} y$ nich we have presented in Sect. 2. Thus inste 1 of getting a $(4 \times 4)$ square matrix (when both sectors are nsidesed) we have a $(4 \times 2)$ matrix which appear in the sorution of the field equation as:

$\widetilde{\chi^{I}}=\frac{1}{\mathcal{N}_{p}} \widetilde{\mathcal{M}}_{\mathcal{J}}^{I} \widetilde{\mathcal{P}}^{\mathcal{J}}+\underset{{ }_{n=0}}{\infty}{\widetilde{\Upsilon_{n,(n)}}}_{\left.\mathcal{M}_{(n)}\right)^{I}} \widetilde{\mathcal{P}}_{(n)}^{\mathcal{J}}$,

where the index $\mathcal{J}, 1,2$ is appearing for the contribution from region $\mathbf{L}$. To write down the total solution in region $\mathbf{L}$ we defin e selld ying matrices:

$$
\begin{aligned}
& \widetilde{\mathcal{N}}^{(}\left(\begin{array}{ll}
\alpha_{\mathbf{L}}^{\sigma} & \beta_{\mathbf{L}^{\sigma}}^{\sigma} \\
\beta_{\mathbf{L}}^{\sigma^{*}} & \alpha_{\mathbf{L}}^{\sigma^{*}}
\end{array}\right), \\
& \left(\widetilde{\left.\mathcal{M}_{(n)}\right)_{\mathcal{J}}^{I}}=\left(\begin{array}{cc}
\bar{\alpha}_{\mathbf{L}, n}^{\sigma} & \bar{\beta}_{\mathbf{L}, n}^{\sigma} \\
\bar{\beta}_{\mathbf{L}, n}^{\sigma^{*}} & \bar{\alpha}_{\mathbf{L}, n}^{\sigma^{*}}
\end{array}\right)\right. \text {, } \\
& \widetilde{\chi^{I}}=\left(\begin{array}{c}
\chi^{\sigma}(t) \\
\chi^{\sigma *}(t),
\end{array}\right), \quad \widetilde{\mathcal{P}}^{\mathcal{J}}=\left(\begin{array}{c}
\widetilde{\mathcal{P}}^{\mathbf{L}} \\
\widetilde{\mathcal{P}}^{\mathbf{L}^{*}},
\end{array}\right), \\
& \widetilde{\mathcal{P}}_{(n)}^{\mathcal{J}}=\left(\begin{array}{c}
\widetilde{\mathcal{P}}^{\mathbf{L}, n} \\
\widetilde{\mathcal{P}}^{\mathbf{L}^{*}, n}
\end{array}\right),
\end{aligned}
$$

where $\sigma= \pm 1, I=1,2,3,4$ and $\mathcal{J}=1,2$. The Fourier mode of the field operator, which is also the total solution of the field equation for axion (in presence of source contribution) can be expressed as:

$$
\begin{aligned}
\widetilde{\Phi\left(t_{\mathbf{L}}\right)}= & \frac{H}{\sinh t_{\mathbf{L}}} Q_{I}{\widetilde{\chi^{I}}}^{I}=\frac{H}{\sinh t_{\mathbf{L}}} Q_{I} \\
& \times\left[\frac{1}{\mathcal{N}_{p}} \widetilde{\mathcal{M}}_{\mathcal{J}}^{I} \widetilde{\mathcal{P}}^{J}+\sum_{n=0}^{\infty} \frac{1}{\mathcal{N}_{p,(n)}}\left(\mathcal{M}_{(n)} \tilde{\mathcal{P}}_{(n)}\right)\right.
\end{aligned}
$$

where the operator $Q_{I}$ repres nt a set of creation and annihilation operators which re c ned (n Sect. 2) for Bunch Davies vacuum $(\alpha=0)$ an. vacua $(\alpha \neq 0)$ as:

$Q_{I} \equiv\left\{\begin{array}{c}a_{I}=\left(a_{\sigma}, a_{\sigma}^{\dagger}\right)=r_{y}^{(c)}+\sum_{n=0}^{\infty} a_{I(n)}^{(p)} \\ \text { for unch Davies vacuum } \\ \left.d_{I}=\quad \begin{array}{l}\left.d_{\sigma}^{\dagger}\right)=\left[d_{I}^{(c)}+\sum_{n=0}^{\infty} d_{I(n)}^{(p)}\right.\end{array}\right] \\ \text { for vacua. }\end{array}\right.$

Here we ave labeled the time coordinate $t$ by $t_{\mathbf{L}}$ since we e considering the left region $\mathbf{L}$ only.

To explicitly write down the expression for the amplitude of the normalized power spectrum, we start with the column matrix representation of the time dependent part of the solution of the wave function, given by:

$$
\begin{aligned}
& \widetilde{\chi^{I}}=\left(\begin{array}{c}
\chi^{\sigma}(t) \\
\chi^{\sigma *(t)}
\end{array}\right)=\left(\begin{array}{c}
\mathcal{A}_{\mathbf{L}}^{\sigma} \widetilde{\mathcal{P}}^{\mathbf{L}}+\mathcal{B}_{\mathbf{L}}^{\sigma} \widetilde{\mathcal{P}}^{\mathbf{L} *} \\
\mathcal{B}_{\mathbf{L}}^{\sigma *} \widetilde{\mathcal{P}}^{\mathbf{L}}+\mathcal{A}_{\mathbf{L}}^{\sigma *} \widetilde{\mathcal{P}}^{\mathbf{L} *}
\end{array}\right) \\
& +\sum_{n=0}^{\infty}\left(\begin{array}{lll}
\mathcal{A}_{\mathbf{L},(n)}^{\sigma} & \widetilde{\mathcal{P}}_{(n)}^{\mathbf{L}}+\mathcal{B}_{\mathbf{L},(n)}^{\sigma} & \widetilde{\mathcal{P}}_{(n)}^{\mathbf{L} *} \\
\mathcal{B}_{\mathbf{L},(n)}^{\sigma *} & \widetilde{\mathcal{P}}_{(n)}^{\mathbf{L}}+\mathcal{A}_{\mathbf{L},(n)}^{\sigma *} & \widetilde{\mathcal{P}}_{(n)}^{\mathbf{L} *}
\end{array}\right),
\end{aligned}
$$

where the entries of the column matrix for the complementary and particular integral part of the solution are given by the following expressions:

$$
\begin{aligned}
\mathcal{A}_{\mathbf{L}}^{\sigma} & =\frac{\alpha_{\mathbf{L}}^{\sigma}}{\mathcal{N}_{p}}=\sigma \frac{e^{\pi p}-i \sigma e^{-i \pi v}}{\mathcal{N}_{p} \Gamma\left(v+i p+\frac{1}{2}\right)}, \\
\mathcal{B}_{\mathbf{L}}^{\sigma} & =\frac{\beta_{\mathbf{L}}^{\sigma}}{\mathcal{N}_{p}}=-\sigma \frac{e^{-\pi p}-i \sigma e^{-i \pi v}}{\mathcal{N}_{p} \Gamma\left(v-i p+\frac{1}{2}\right)}, \\
\mathcal{A}_{\mathbf{L},(n)}^{\sigma} & =\frac{\alpha_{\mathbf{L},(\mathbf{n})}^{\sigma}}{\mathcal{N}_{p,(n)}}=\sigma \frac{e^{\pi p_{n}}-i \sigma e^{-i \pi v}}{\mathcal{N}_{p,(n)} \Gamma\left(v+i p_{n}+\frac{1}{2}\right)}, \\
\mathcal{B}_{\mathbf{L},(n)}^{\sigma} & =\frac{\beta_{\mathbf{L},(n)}^{\sigma}}{\mathcal{N}_{p,(n)}}=-\sigma \frac{e^{-\pi p_{n}}-i \sigma e^{-i \pi v}}{\mathcal{N}_{p,(n)} \Gamma\left(v-i p_{n}+\frac{1}{2}\right)} .
\end{aligned}
$$

$\mathcal{N}_{p}$ and $\mathcal{N}_{p,(n)}$ in the above equations are the normalization constants for the complementary part and particular integral part of the solution as defined Sect. 2. 


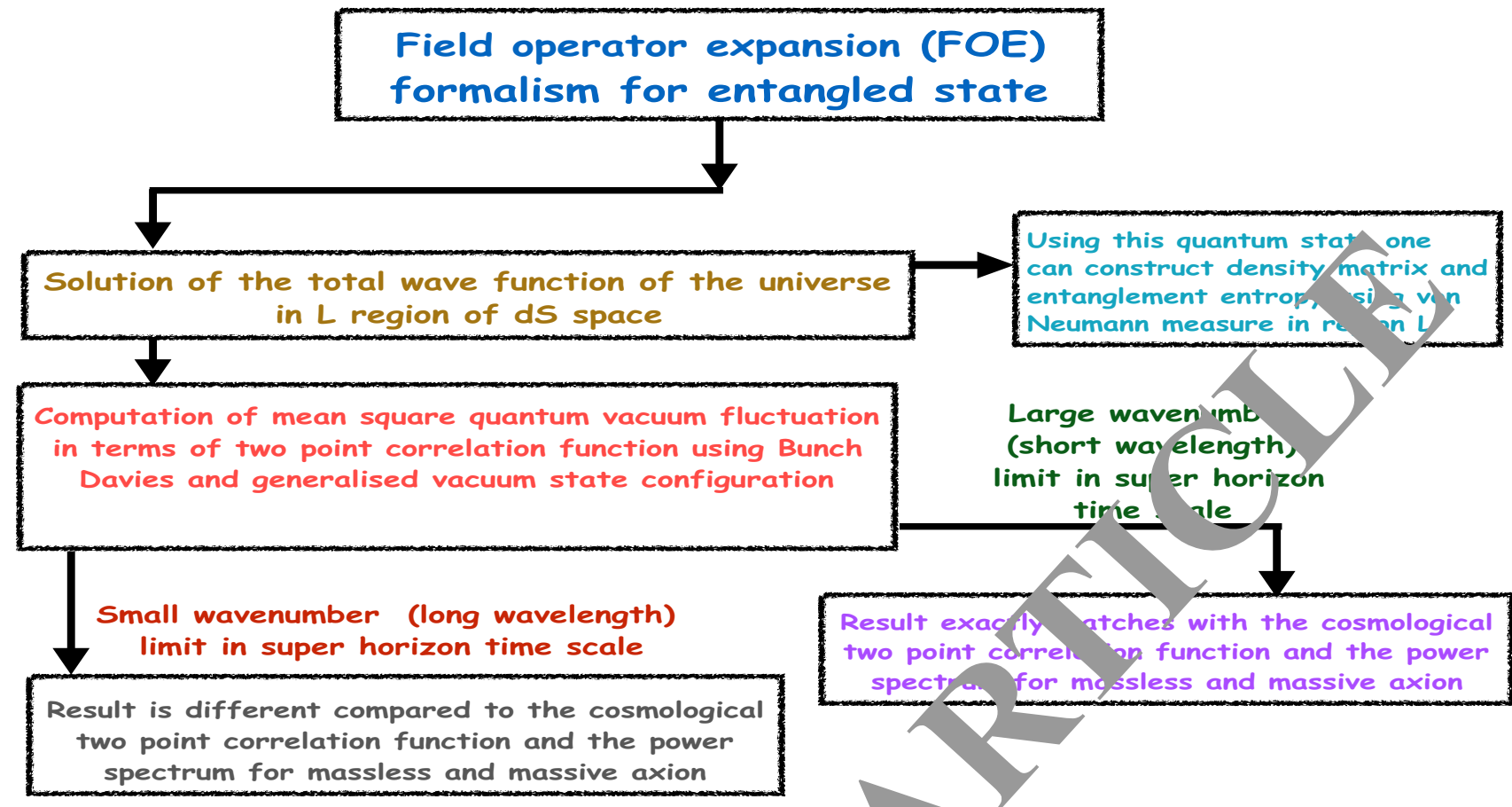

Fig. 5 Schematic diagram for the computation algorithm of field operator expansion netr od for entangled state of axion in de Sitter hyperbolic open chart

\subsubsection{Two point correlation function}

To compute the expression for the two point correla $n \mathrm{f}$ nction for the vacuum fluctuation let us now con ontra. on a single mode with fixed value of the $\mathbf{S O}(3,1)$ antum nu, nbers $p, l$ and $m$. As a result the mean square $v_{a}$.Im fluctuation of axion for any generalized a oitrary vacu $/ \mathrm{m}$ state $(|\Omega\rangle)$ can be expressed as:

$$
\begin{aligned}
& \left\langle\Omega\left|\widetilde{\Phi_{p l m}\left(t_{\mathbf{L}}\right)}\left(\widetilde{\Phi_{p^{\prime} l^{\prime} m^{\prime}}\left(t_{\mathbf{L}}\right)}\right)^{\dagger}\right|^{-}\right. \\
& =\frac{H^{2}}{\sinh ^{2} t_{\mathbf{L}}}\langle\Omega|\left[Q_{I} \widetilde{\left.\gamma_{\lrcorner^{\prime}}\right]}\left(\left[Q_{L} \widetilde{\chi^{I}}\right]_{p^{\prime} l^{\prime} m^{\prime}}\right)^{\dagger}|\Omega\rangle\right. \text {. }
\end{aligned}
$$

Further explicit $/ y$ riting thy expression for the mean square vacuum fluctuation axion for Bunch Davies vacuum we get the for owing simpified expressions:

$$
\begin{aligned}
& \langle\mathbf{B D}| \Phi_{p l m} \\
& =\frac{H^{2}}{\sinh ^{2} t_{\mathbf{L}}} \sum_{\sigma= \pm 1}\left|\widetilde{\chi^{\sigma}}\right|^{2} \delta\left(p-p^{\prime}\right) \delta_{l l^{\prime}} \delta_{m m^{\prime}} \\
& \equiv P_{\mathbf{B D}}\left(p, t_{\mathbf{L}}\right) \delta\left(p-p^{\prime}\right) \delta_{l l^{\prime}} \delta_{m m^{\prime}},
\end{aligned}
$$

$$
\begin{aligned}
\mathcal{P}_{\mathbf{B D}}\left(p, t_{\mathbf{L}}\right) & =\frac{p^{3}}{2 \pi^{2}} P_{\mathbf{B D}}\left(p, t_{\mathbf{L}}\right) \\
& =\frac{p^{3}}{2 \pi^{2}} \frac{H^{2}}{\sinh ^{2} t_{\mathbf{L}}} \sum_{\sigma= \pm 1}\left|\widetilde{\chi^{\sigma}}\right|^{2} .
\end{aligned}
$$

Further using Eq. (3.6) we compute the following expression, which is appearing in the expression for the amplitude of the normalized power spectrum:

$$
\begin{aligned}
& \sum_{\sigma= \pm 1}\left|\widetilde{\chi^{\sigma}}\right|^{2}=\sum_{\sigma= \pm 1}\left(\widetilde{\chi^{\sigma}}\right)^{\dagger} \widetilde{\chi^{\sigma}} \\
& =\left[\left(\left|\mathcal{A}_{\mathbf{L}}^{\sigma}\right|^{2}+\left|\mathcal{B}_{\mathbf{L}}^{\sigma}\right|^{2}\right) \widetilde{\mathcal{P}}^{\mathbf{L}} \widetilde{\mathcal{P}}^{\mathbf{L} *}\right. \\
& +\mathcal{A}_{\mathbf{L}}^{\sigma} \mathcal{B}_{\mathbf{L}}^{\sigma *}\left(\widetilde{\mathcal{P}}^{\mathbf{L}}\right)^{2}+\mathcal{A}_{\mathbf{L}}^{\sigma *} \mathcal{B}_{\mathbf{L}}^{\sigma}\left(\widetilde{\mathcal{P}}^{\mathbf{L} *}\right)^{2} \\
& +\sum_{n=0}^{\infty}\left\{\left(\mathcal{A}_{\mathbf{L}}^{\sigma} \mathcal{A}_{\mathbf{L},(n)}^{\sigma *}+\mathcal{B}_{\mathbf{L}}^{\sigma} \mathcal{B}_{\mathbf{L},(n)}^{\sigma *}\right) \widetilde{\mathcal{P}}^{\mathbf{L}} \widetilde{\mathcal{P}}_{(n)}^{\mathbf{L} *}\right. \\
& +\left(\mathcal{A}_{\mathbf{L}}^{\sigma} \mathcal{B}_{\mathbf{L},(n)}^{\sigma *}+\mathcal{A}_{\mathbf{L},(n)}^{\sigma} \mathcal{B}_{\mathbf{L}}^{\sigma *}\right) \widetilde{\mathcal{P}}^{\mathbf{L}} \widetilde{\mathcal{P}}_{(n)}^{\mathbf{L}} \\
& \left.+\left(\mathcal{A}_{\mathbf{L},(n)}^{\sigma *} \mathcal{B}_{\mathbf{L}}^{\sigma}+\mathcal{A}_{\mathbf{L}}^{\sigma *} \mathcal{B}_{\mathbf{L},(n)}^{\sigma}\right) \widetilde{\mathcal{P}}_{(n)}^{\mathbf{L} *} \widetilde{\mathcal{P}}^{\mathbf{L} *}\right\} \\
& +\sum_{n=0}^{\infty} \sum_{m=0}^{\infty}\left\{\left(\mathcal{A}_{\mathbf{L},(n)}^{\sigma} \mathcal{A}_{\mathbf{L},(m)}^{\sigma *}+\mathcal{B}_{\mathbf{L},(n)}^{\sigma} \mathcal{B}_{\mathbf{L},(m)}^{\sigma *}\right) \widetilde{\mathcal{P}}_{(n)}^{\mathbf{L}} \widetilde{\mathcal{P}}_{(m)}^{\mathbf{L} *}\right. \\
& \left.\left.+\mathcal{A}_{\mathbf{L},(n)}^{\sigma} \mathcal{B}_{\mathbf{L},(m)}^{\sigma *} \widetilde{\mathcal{P}}_{(n)}^{\mathbf{L}} \widetilde{\mathcal{P}}_{(m)}^{\mathbf{L}}+\mathcal{A}_{\mathbf{L},(n)}^{\sigma *} \mathcal{B}_{\mathbf{L},(m)}^{\sigma} \widetilde{\mathcal{P}}_{(n)}^{\mathbf{L} *} \widetilde{\mathcal{P}}_{(m)}^{\mathbf{L} *}\right\}\right] .
\end{aligned}
$$


Using Eq. (3.14), the amplitude of the normalized power spectrum of axion from Bunch Davies vacuum can be expressed in all time scales of region $\mathbf{L}$ as:

$$
\begin{aligned}
& \mathcal{P}_{\mathbf{B D}}\left(p, t_{\mathbf{L}}\right)=\frac{p^{3}}{2 \pi^{2}} \frac{H^{2}}{\sinh ^{2} t_{\mathbf{L}}} \sum_{\sigma= \pm 1}\left|\widetilde{\chi^{\sigma}}\right|^{2}=\frac{p^{3}}{2 \pi^{2}} \frac{H^{2}}{\sinh ^{2} t_{\mathbf{L}}} \\
& \times\left[\left(\left|\mathcal{A}_{\mathbf{L}}^{\sigma}\right|^{2}+\left|\mathcal{B}_{\mathbf{L}}^{\sigma}\right|^{2}\right) \widetilde{\mathcal{P}}^{\mathbf{L}} \widetilde{\mathcal{P}}^{\mathbf{L} *}+\mathcal{A}_{\mathbf{L}}^{\sigma} \mathcal{B}_{\mathbf{L}}^{\sigma *}\left(\widetilde{\mathcal{P}}^{\mathbf{L}}\right)^{2}\right. \\
& +\mathcal{A}_{\mathbf{L}}^{\sigma *} \mathcal{B}_{\mathbf{L}}^{\sigma}\left(\widetilde{\mathcal{P}}^{\mathbf{L} *}\right)^{2} \\
& +\sum_{n=0}^{\infty}\left\{\left(\mathcal{A}_{\mathbf{L}}^{\sigma} \mathcal{A}_{\mathbf{L},(n)}^{\sigma *}+\mathcal{B}_{\mathbf{L}}^{\sigma} \mathcal{B}_{\mathbf{L},(n)}^{\sigma *}\right) \widetilde{\mathcal{P}}^{\mathbf{L}} \widetilde{\mathcal{P}}_{(n)}^{\mathbf{L} *}\right. \\
& +\left(\mathcal{A}_{\mathbf{L}}^{\sigma} \mathcal{B}_{\mathbf{L},(n)}^{\sigma *}+\mathcal{A}_{\mathbf{L},(n)}^{\sigma} \mathcal{B}_{\mathbf{L}}^{\sigma *}\right) \widetilde{\mathcal{P}}^{\mathbf{L}} \widetilde{\mathcal{P}}_{(n)}^{\mathbf{L}} \\
& \left.+\left(\mathcal{A}_{\mathbf{L},(n)}^{\sigma *} \mathcal{B}_{\mathbf{L}}^{\sigma}+\mathcal{A}_{\mathbf{L}}^{\sigma *} \mathcal{B}_{\mathbf{L},(n)}^{\sigma}\right) \widetilde{\mathcal{P}}_{(n)}^{\mathbf{L} *} \widetilde{\mathcal{P}}^{\mathbf{L} *}\right\} \\
& +\sum_{n=0}^{\infty} \sum_{m=0}^{\infty}\left\{\left(\mathcal{A}_{\mathbf{L},(n)}^{\sigma} \mathcal{A}_{\mathbf{L},(m)}^{\sigma *}+\mathcal{B}_{\mathbf{L},(n)}^{\sigma} \mathcal{B}_{\mathbf{L},(m)}^{\sigma *}\right) \widetilde{\mathcal{P}}_{(n)}^{\mathbf{L}} \widetilde{\mathcal{P}}_{(m)}^{\mathbf{L} *}\right. \\
& \left.\left.+\mathcal{A}_{\mathbf{L},(n)}^{\sigma} \mathcal{B}_{\mathbf{L},(m)}^{\sigma *} \widetilde{\mathcal{P}}_{(n)}^{\mathbf{L}} \widetilde{\mathcal{P}}_{(m)}^{\mathbf{L}}+\mathcal{A}_{\mathbf{L},(n)}^{\sigma *} \mathcal{B}_{\mathbf{L},(m)}^{\sigma} \widetilde{\mathcal{P}}_{(n)}^{\mathbf{L} *} \widetilde{\mathcal{P}}_{(m)}^{\mathbf{L} *}\right\}\right] .
\end{aligned}
$$

$$
\begin{aligned}
& \widetilde{\mathcal{M}(p, v)}=\frac{2^{2 v-1}(\Gamma(v))^{2}}{\pi} \sum_{\sigma= \pm 1}\left[\frac{\left(\left|\mathcal{A}_{\mathbf{L}}^{\sigma}\right|^{2}+\left|\mathcal{B}_{\mathbf{L}}^{\sigma}\right|^{2}\right)}{\left|\Gamma\left(v+i p+\frac{1}{2}\right)\right|^{2}}\right. \\
& +\frac{\mathcal{A}_{\mathbf{L}}^{\sigma} \mathcal{B}_{\mathbf{L}}^{\sigma *}}{\left(\Gamma\left(v-i p+\frac{1}{2}\right)\right)^{2}}+\frac{\mathcal{A}_{\mathbf{L}}^{\sigma *} \mathcal{B}_{\mathbf{L}}^{\sigma}}{\left(\Gamma\left(v+i p+\frac{1}{2}\right)\right)^{2}} \\
& +\sum_{n=0}^{\infty}\left\{\frac{\left(\mathcal{A}_{\mathbf{L}}^{\sigma} \mathcal{A}_{\mathbf{L},(n)}^{\sigma *}+\mathcal{B}_{\mathbf{L}}^{\sigma} \mathcal{B}_{\mathbf{I}}^{\sigma *(n)}\right)}{\Gamma\left(v-i p+\frac{1}{2}\right) \Gamma(\nu}\right. \\
& +\frac{\left(\mathcal{A}_{\mathbf{L}}^{\sigma} \mathcal{B}_{\mathbf{L},(n)}^{\sigma *}+\mathcal{A}_{\mathbf{L}}^{\sigma}\left(\mathcal{B}_{\mathbf{L}}^{\sigma *}\right)\right.}{\left.\Gamma\left(v-i p+\frac{1}{2}\right) \Gamma / \iota_{\Lambda}+\frac{1}{2}\right)} \\
& \left.+\frac{\left(\mathcal{A}_{\mathbf{L},(n)}^{\sigma *} \mathcal{B}_{\mathbf{L}}^{\sigma}-\mathcal{A}_{\mathbf{L}}^{\sigma *} \mathcal{B}_{\mathbf{I}}^{\sigma},(n)\right.}{\Gamma\left(v+i_{p m}\right.} \frac{\left.\frac{1}{2}\right)}{\left.+i p+\frac{1}{2}\right)}\right\} \\
& +\sum_{j=0}^{\infty} \sum_{m=0} \frac{\left(\mathcal{A}_{\mathbf{L},(n)} \mathcal{A}_{\mathbf{L},(m)}^{\sigma *}+\mathcal{B}_{\mathbf{L},(n)}^{\sigma} \mathcal{B}_{\mathbf{L},(m)}^{\sigma *}\right)}{\left(v-i p_{n}+\frac{1}{2}\right) \Gamma\left(v+i p_{m}+\frac{1}{2}\right)} \\
& \frac{\mathcal{A}_{\mathbf{L},(n)}^{\sigma} \mathcal{B}_{\mathbf{L},(m)}^{\sigma *}}{\left.Y-i p_{n}+\frac{1}{2}\right) \Gamma\left(v-i p_{m}+\frac{1}{2}\right)} \\
& \left.\left.4 \frac{\mathcal{A}_{\mathbf{L},(n)}^{\sigma *} \mathcal{B}_{\mathbf{L},(m)}^{\sigma}}{\Gamma\left(v+i p_{n}+\frac{1}{2}\right) \Gamma\left(v+i p_{m}+\frac{1}{2}\right)}\right\}\right] \text {. }
\end{aligned}
$$

However, it is not easy to extract any informatio from Eq. (3.15) for cosmological predictions. Hence, we on ider the superhorizon time scales $\left(t_{\mathbf{L}}>>1\right)$ of regis.. $\mathbf{L}$. a case, the Legendre functions, appearing in $t^{\prime}$ 'omplem, $n$ tary part and the particular integral part of the tim spendent solution, can be approximated as:

$$
\begin{aligned}
& \left(\widetilde{\mathcal{P}}^{\mathbf{L}}, \widetilde{\mathcal{P}}^{\mathbf{L} *}\right) \equiv P_{\nu-\frac{1}{2}}^{ \pm i p}\left(\cosh t_{\mathbf{L}}\right) \\
& \stackrel{t_{L}>>1}{\longrightarrow} \frac{2^{v-\frac{1}{2}}(\cosh t 2)^{v-\frac{1}{2} \Gamma(v)}}{\sqrt{t_{2}} \Gamma\left(v \mp \ell p+\frac{1}{2}\right)},
\end{aligned}
$$

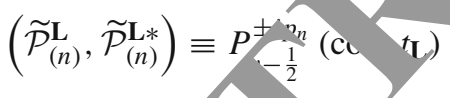

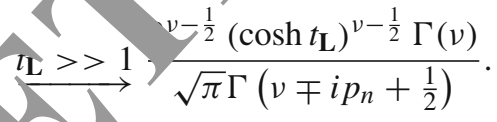

Conse ently, 1 the superhorizon time scales $\left(t_{\mathbf{L}}>>1\right)$ of 16. $n$ (3.14) can be further simplified as:

$$
\begin{aligned}
& \sum_{\sigma= \pm 1}\left|\tilde{\chi}^{\sigma}\right|^{2}=\sum_{\sigma= \pm 1}\left(\widetilde{\chi^{\sigma}}\right)^{\dagger} \widetilde{\chi^{\sigma}} \\
& \stackrel{t_{\mathbf{L}}>1}{\longrightarrow} \widetilde{\mathcal{M ( p , v})}\left(\cosh t_{\mathbf{L}}\right)^{2 v-1}
\end{aligned}
$$

where the time independent function $\widetilde{\mathcal{M}(p, v)}$ is defined as:
A. a result, in the superhorizon time scales $\left(t_{\mathbf{L}}>>1\right)$ of egion $\mathbf{L}$ the amplitude of the normalized power spectrum of axion from Bunch Davies vacuum can be expressed as:

$$
\begin{aligned}
& \mathcal{P}_{\mathbf{B D}}\left(p, t_{\mathbf{L}}\right)=\frac{p^{3}}{2 \pi^{2}} \frac{H^{2}}{\sinh ^{2} t_{\mathbf{L}}} \sum_{\sigma= \pm 1}\left|\widetilde{\chi^{\sigma}}\right|^{2} \\
&\left.\stackrel{t_{\mathbf{L}}}{ }>>1 \frac{p^{3}}{2 \pi^{2}}\left(\cosh t_{\mathbf{L}}\right)^{2 \nu-3} H^{2} \widetilde{\mathcal{M ( p , \nu}}\right)
\end{aligned}
$$

Here, it is important to note that in the superhorizon time scales $\left(t_{\mathbf{L}}>>1\right)$ of region $\mathbf{L}$ if we consider the massless case where we fix the mass parameter to be $v=3 / 2$, then the time dependent contribution can be approximated as:

$$
\left(\frac{\left(\cosh t_{\mathbf{L}}\right)^{2 v-1}}{\sinh ^{2} t_{\mathbf{L}}}\right)_{\nu=3 / 2} \stackrel{t_{\mathbf{L}}>>1}{\longrightarrow} 1 .
$$

Consequently, in the superhorizon time scales of region $\mathbf{L}$ and for the massless axion case, the amplitude of the normalized power spectrum of axion from Bunch Davies vacuum can be expressed as:

$$
\begin{aligned}
\mathcal{P}_{\mathbf{B D}}\left(p, t_{\mathbf{L}}\right)= & \frac{p^{3}}{2 \pi^{2}} \frac{H^{2}}{\sinh ^{2} t_{\mathbf{L}}} \sum_{\sigma= \pm 1}\left|\widetilde{\chi^{\sigma}}\right|^{2} \\
& \stackrel{t_{\mathbf{L}}>>1, v=3 / 2}{\longrightarrow} \frac{p^{3}}{2 \pi^{2}} H^{2} \mathcal{M}(\widetilde{p, v=3} / 2) .
\end{aligned}
$$


This implies that in the massless case, the amplitude of the vacuum fluctuation gets frozen with respect to the time scale when the associated modes exit the horizon.

Further to infer the exact wave number dependence of the amplitude of the normalized power spectrum from Bunch Davies vacuum we need to know the behaviour of the power spectrum at very short wavelengths $\left(p, p_{n}>>1\right)$. In this limit it is expected that the power spectrum should match the result obtained for spatially flat universe. Note that in the short wave length approximation the time independent function $\mathcal{M}(\overrightarrow{p>>} 1, v)$ for any arbitrary mass parameter $v$ can be expressed as:

$\mathcal{M}(\widetilde{p>>} 1, v)=\frac{2^{2(v-1)}(\Gamma(v))^{2}}{p^{3} \pi} \mathcal{G}(\widetilde{p>>} 1)$,

where we have defined a new function $\mathcal{G}(\widetilde{p>>} 1)$ in the short wave length limit as:

$$
\begin{aligned}
\widetilde{\mathcal{G}(p)}= & \frac{1}{\left(1+\frac{1}{82944 p^{4}}\right)} \\
& \times\left[\left(1+e^{-2 \pi p}\right)^{2}+\sum_{n=0}^{\infty}\left(\frac{p}{p_{n}}\right)^{\frac{3}{2}} \frac{\sqrt{1+\frac{1}{82944 p^{4}}}}{\sqrt{1+\frac{1}{82944 p_{n}^{4}}}}\right. \\
& \times\left(1+2\left(e^{-2 \pi p}+e^{-2 \pi p_{n}}\right)+e^{-2 \pi\left(p+p_{n}\right)}\right) \\
& \left.+\sum_{n=0}^{\infty} \sum_{m=0}^{\infty} \frac{p^{3}}{\left(p_{n} p_{m}\right)^{3 / 2}} \times \frac{\left(1+\frac{1}{8294 p^{4}}\right.}{\sqrt{1+\frac{1}{82944}}}\right) \\
& \left.\times\left(1+e^{-\pi\left(p_{m}+p_{n}\right)}\right)^{2}\right] .
\end{aligned}
$$

The above equation implies thatros large $p, p_{n}>>1$ one can rewrite this as, $\widetilde{\mathcal{G}} \sim 1 \neg \cdots$, and all the $\cdots$ terms can be considered as sr $11 \mathrm{cc}$ rection terms. Also for the mass less case $(v=3 / 2)$ nd . he snort wave length approximation, the time in. endent anction $\mathcal{M}(\widetilde{p, v=3 / 2})$ can be further simplifred a

$\mathcal{M}\left(p>>>(\widetilde{\mathcal{p}>>})=\frac{\mathcal{G})}{2 p^{3}}\right.$.

$\Gamma_{1} \quad{ }_{1}, \quad$ superhorizon time scales $\left(t_{\mathbf{L}}>>1\right)$ of region $\mathbf{L}$, th nplitude of the normalized power spectrum of axion from Bunch Davies vacuum in the short wave length limit can be expressed as:

$$
\begin{aligned}
& \mathcal{P}_{\mathbf{B D}}\left(p>>1, t_{\mathbf{L}}>>1\right) \\
& =\frac{p^{3}}{2 \pi^{2}}\left(\cosh t_{\mathbf{L}}\right)^{2 v-3} H^{2} \widetilde{\mathcal{M}(p, v)}
\end{aligned}
$$

$$
=\left(2 \cosh t_{\mathbf{L}}\right)^{2 \nu-3}\left(\frac{H}{2 \pi}\right)^{2}\left(\frac{\Gamma(\nu)}{\Gamma\left(\frac{3}{2}\right)}\right)^{2} \mathcal{G}(\widetilde{p>>} 1) .
$$

Also for the massless case $(v=3 / 2)$ in the superhorizon time scales $\left(t_{\mathbf{L}}>>1\right)$ of region $\mathbf{L}$ the amplitude of $t^{\operatorname{th}}$ normalized power spectrum of axion from Bunch Davi s vecuum in the short wave length limit can be simplified as.

$$
\begin{aligned}
\mathcal{P}_{\mathbf{B D}}\left(p>>1, t_{\mathbf{L}}>>1\right) & =\frac{p^{3}}{2 \pi^{2}} H \cdot(p>>1, v=3 / 2) \\
& =\left(\frac{H}{G}\right)^{2} \mathcal{G}(p>>1) .
\end{aligned}
$$

Now, we generaline above results for the two point correlation fu tion and, $\mathcal{C}$ associated power spectrum for $\alpha$ vacua. For $x$ va the mean square vacuum fluctuation of axion e short ave length limit can be expressed as:

$$
\begin{aligned}
& \left\langle\alpha\left|\Phi_{p}\left(\Phi_{p^{\prime} m^{\prime}}\left(t_{\mathbf{L}}\right)\right)^{\dagger}\right| \alpha\right\rangle \\
& =\frac{\sinh _{t_{\mathbf{L}}}}{2}\left\langle\alpha\left|\left[d_{I} \widetilde{\chi^{I}}\right]_{p l m}\left(\left[d_{I} \widetilde{\chi^{I}}\right]_{p^{\prime} l^{\prime} m^{\prime}}\right)^{\dagger}\right| \alpha\right\rangle \\
& =\frac{H^{2}}{\sinh ^{2} t_{\mathbf{L}}} \sum_{\sigma= \pm 1}\left|\widetilde{\chi^{\sigma}}\right|^{2} \delta\left(p-p^{\prime}\right) \delta_{l l^{\prime}} \delta_{m m^{\prime}} \\
& \equiv P\left(p>>1, \alpha, t_{\mathbf{L}}\right) \delta\left(p-p^{\prime}\right) \delta_{l l^{\prime}} \delta_{m m^{\prime}} .
\end{aligned}
$$

where we have defined the amplitude of the normalized power spectrum of axion in the short wave length limit as:

$$
\begin{aligned}
\mathcal{P} & \left(p>>1, \alpha, t_{\mathbf{L}}\right) \\
& =\frac{p^{3}}{2 \pi^{2}} P\left(p>>1, \alpha, t_{\mathbf{L}}\right) \\
& =P_{\mathbf{B D}}\left(p>>1, t_{\mathbf{L}}\right)(\cosh 2 \alpha-\sinh 2 \alpha) \\
& =\exp (-2 \alpha) P_{\mathbf{B D}}\left(p>>1, t_{\mathbf{L}}\right) .
\end{aligned}
$$

In the above equation, $P_{\mathbf{B D}}\left(p, t_{\mathbf{L}}\right)$ is defined as:

$$
P_{\mathbf{B D}}\left(p>>1, t_{\mathbf{L}}\right)=\frac{p^{3}}{2 \pi^{2}} \frac{H^{2}}{\sinh ^{2} t_{\mathbf{L}}} \sum_{\sigma= \pm 1}\left|\widetilde{\chi^{\sigma}}\right|^{2} .
$$

We carry out the same approximations as earlier and we note that in the superhorizon time scales $\left(t_{\mathbf{L}}>>1\right)$ of region $\mathbf{L}$ the amplitude of the normalized power spectrum of axion in the short wave length limit from $\alpha$ vacua can be expressed as:

$$
\begin{aligned}
& \mathcal{P}\left(p>>1, \alpha, t_{\mathbf{L}}>>1\right) \\
& \quad=\mathcal{P}_{\mathbf{B D}}\left(p>>1, t_{\mathbf{L}}>>1\right)(\cosh 2 \alpha-\sinh 2 \alpha) \\
& \quad=\exp (-2 \alpha) \mathcal{P}_{\mathbf{B D}}\left(p, t_{\mathbf{L}}>>1\right),
\end{aligned}
$$




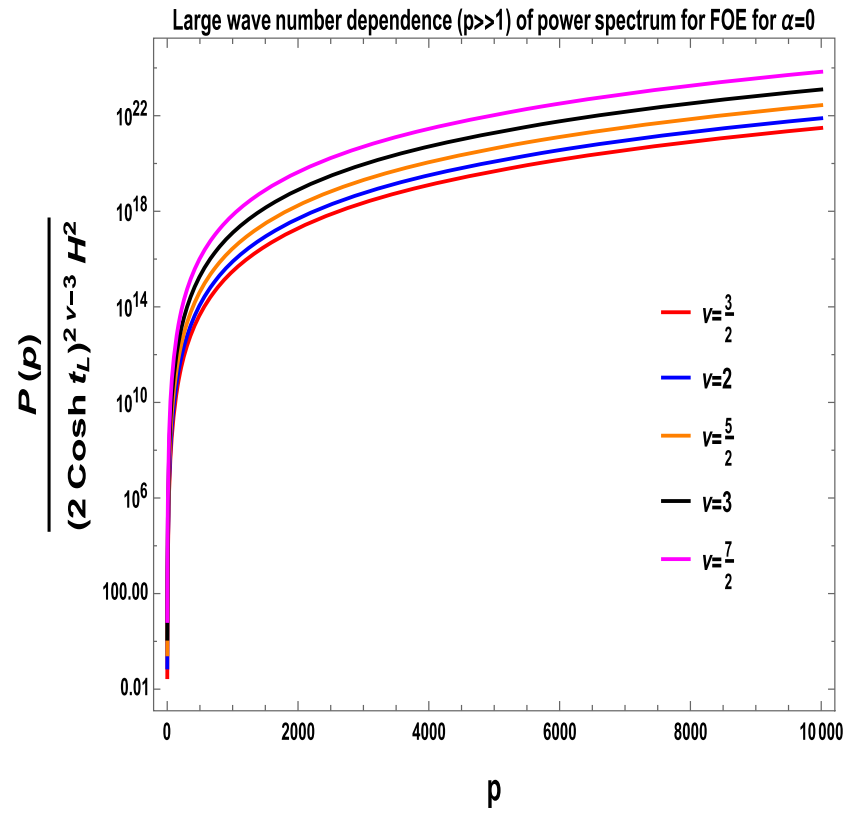

(a) Large wave number dependence of FOE power spectrum for $\alpha=0$.

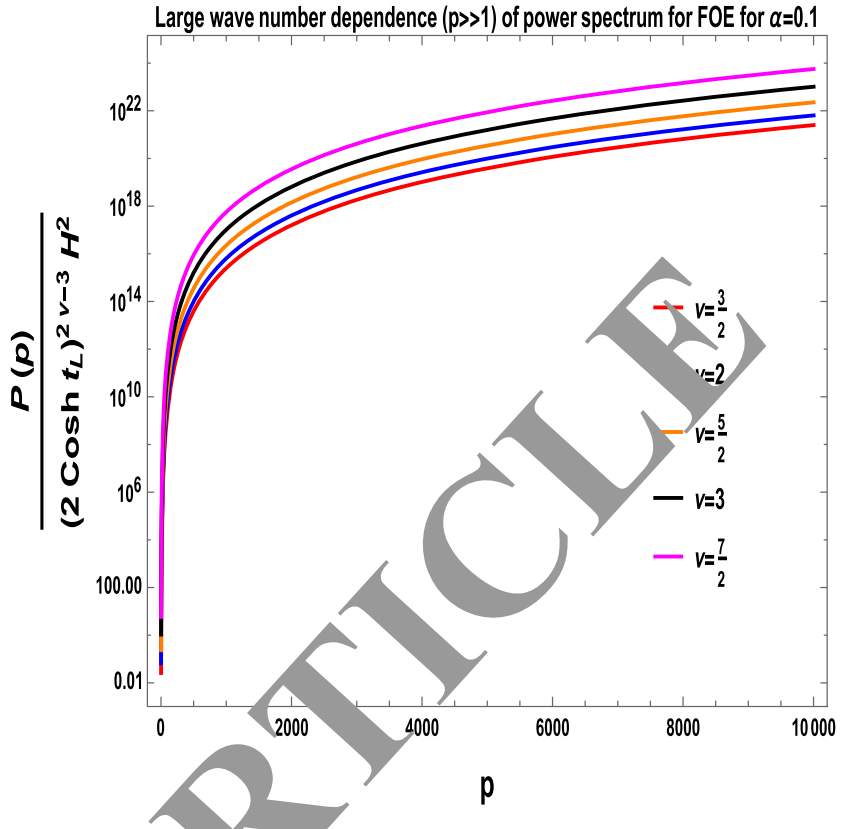

(b) La wave number dependence of FOE power spectrum for $=0.1$

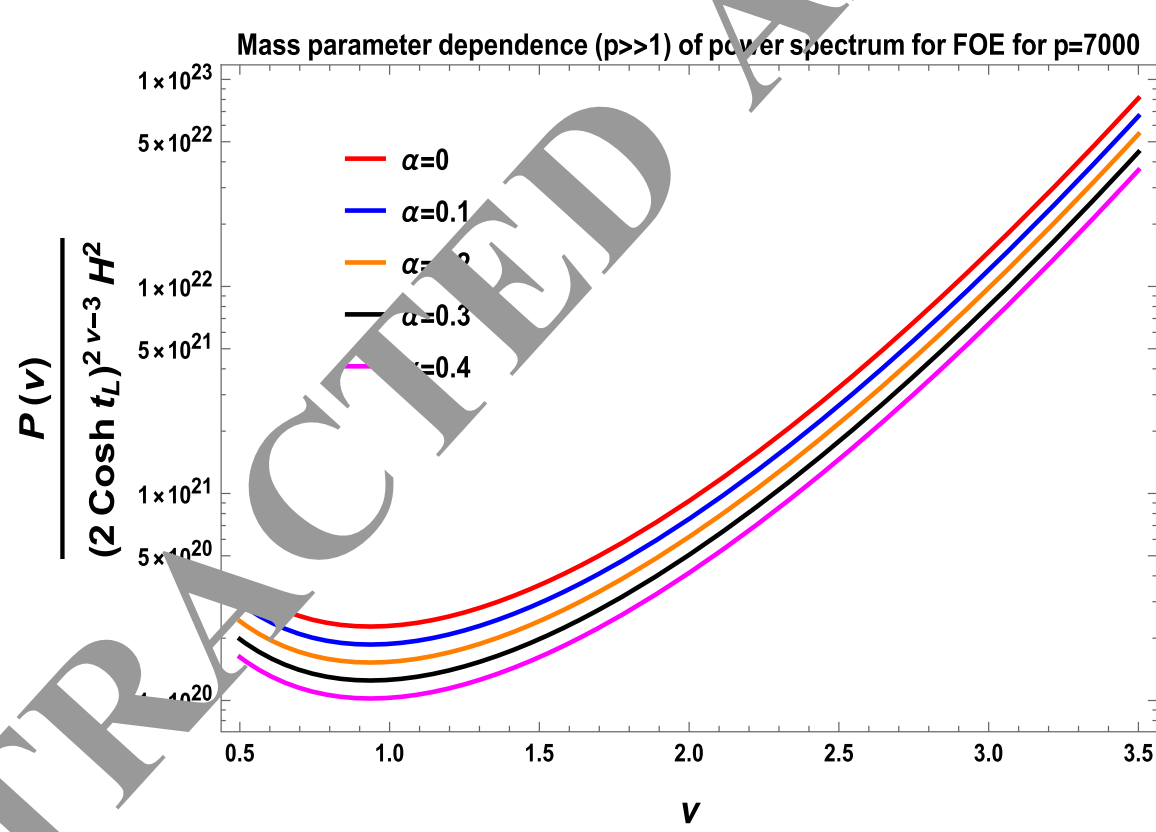

(c) Mass parameter dependence of FOE power spectrum for $p>>1$.

Fig. 6 Feat of $\mathrm{F} J \mathrm{E}$ power spectrum in large wave number region

wh the normalized power spectrum in superhorizon scale for But. n Davies vacuum $\mathcal{P}_{\mathbf{B D}}\left(p>>1, t_{\mathbf{L}}>>1\right)$ is defined in Eq. (3.35). Here it is important to note that, with $\alpha=0$ then we can reproduce the results obtained for Bunch Davies vacuum.

In Fig. 6a, b we have shown the behaviour of the power spectrum of the mean square vacuum fluctuation computed from FOE formalism in the short wave length regime for $\alpha=0$ and $\alpha=0.1$ and for fixed values of the mass parameter $v(=3 / 2,2,5 / 2,3,7 / 2)$ respectively. In both the cases we have found almost similar behaviour. Additionally, in Fig. 6c we have depicted the behaviour of the power spectrum with respect to the mass parameter $v$ with fixed values of the parameter $\alpha(=0,0.1,0.2,0.3,0.4)$. It is clear from this figure that the power spectrum shows two distinct behaviour in $1 / 2<v<1$ and $v>1$ region. For $1 / 2<v<1$ region, 
the amplitude of the normalized power spectrum decreases to a certain value but just after $v=1$ it increases.

On the other hand, to know the exact wavenumber dependence of the amplitude of the normalised power spectrum from Bunch Davies vacuum in the long wavelength limit we need to know the behaviour of the power spectrum at $p, p_{n}<<1$. In this limit it is expected that the power spectrum of axion match with the result obtained for spatially flat universe. Here the time independent function $\mathcal{M}(\widetilde{p<1}, v)$ for any arbitrary mass parameter $v$ can be expressed as:

$\mathcal{M}(\widetilde{p<<1}, v)=\frac{2^{2(\nu-1)}(\Gamma(v))^{2}}{\pi} \mathcal{G}(\widetilde{p<<1})$,

where we have defined a new function $\mathcal{G}(\widetilde{p<<})$ in the long wave length limit as:

$$
\begin{aligned}
& \mathcal{G}(\widetilde{p<} 1)=\frac{\pi}{\left|\Gamma\left(v+\frac{1}{2}\right)\right|^{2}}\left[1+\frac{\left|\Gamma\left(v+\frac{1}{2}\right)\right|^{2}}{\left(\Gamma\left(v+\frac{1}{2}\right)\right)^{2}}\right. \\
& \left.\quad \times\left\{1+3 e^{-\pi p} \sum_{n=0}^{\infty} e^{-\pi p_{n}}+2 \sum_{n=0}^{\infty} \sum_{m=0}^{\infty} e^{-\pi\left(p_{n}+p_{m}\right)}\right\}\right] .
\end{aligned}
$$

This implies that for very small wave numbers $p, p_{n}<<1$ one can write, $\mathcal{G}(\widetilde{p<<}) \sim \frac{\pi}{\left|\Gamma\left(v+\frac{1}{2}\right)\right|^{2}}[1+\cdots]$, where the $\cdots$ terms are small correction terms.

Also for the massless case $(v=3 / 2)$ and in wave length approximation, the time indepe dent fun o $\mathcal{M}(p<<1, v=3 / 2)$ can further be simp fiea

$\mathcal{M}(p<\widetilde{<1, v}=3 / 2)=\frac{\mathcal{G}(\widetilde{p<<} 1}{2}$

Finally, in the super horizon time scales $\left(v_{L}>>1\right)$ of region $\mathbf{L}$ the amplitude of the normaliz u from Bunch Davies vacuum, in th long wave length limit, can be expressed as:

$$
\begin{aligned}
& \mathcal{P}_{\mathbf{B D}}\left(p<<1, t_{\mathrm{I}}>>1\right) \\
& =\frac{p^{3}}{2 \pi^{2}}\left(\sin _{\mathbf{L}}\right)^{2} H^{2} \mathcal{M}(\widetilde{p<<}, v) \\
& =2 \cosh 2^{2 v}-3\left(\frac{H}{2 \pi}\right)^{2} p^{3}\left(\frac{\Gamma(v)}{\Gamma\left(\frac{3}{2}\right)}\right)^{2} \mathcal{G}(\widetilde{p<<} 1),
\end{aligned}
$$

and for the massless case $(v=3 / 2)$ this simplifies to:

$$
\begin{aligned}
& \mathcal{P}_{\mathbf{B D}}\left(p<<1, t_{\mathbf{L}}>>1\right) \\
& =\frac{p^{3}}{2 \pi^{2}} H^{2} \mathcal{M}(p \widetilde{<<1, v}=3 / 2) \\
& =\left(\frac{H}{2 \pi}\right)^{2} p^{3} \mathcal{G}(\widetilde{p<<}) .
\end{aligned}
$$

Here it is important to note that both of Eqs. (3.35) and (3.36) are valid after horizon exit.

Next, we generalize the result for the two point correlation function and the associated power spectrum for $\alpha$ vacua. For $\alpha$ vacua the mean square vacuum fluctuation of axion in the long wave length limit can be expressed as:

$$
\begin{aligned}
& \left\langle\alpha\left|\widetilde{\Phi_{p l m}\left(t_{\mathbf{L}}\right)}\left(\widetilde{\Phi_{p^{\prime} l^{\prime} m^{\prime}}\left(t_{\mathbf{L}}\right)}\right)^{\dagger}\right| \alpha\right\rangle \\
& =\frac{H^{2}}{\sinh ^{2} t_{\mathbf{L}}}\langle\alpha|\left[d_{I} \widetilde{\chi^{I}}\right]_{p l m}\left(\left[d_{I / 2} \widetilde{\tau}{ }_{l^{\prime} m^{\prime}}\right){ }^{\prime}|\alpha\rangle\right. \\
& =\frac{H^{2}}{\sinh ^{2} t_{\mathbf{L}}} \sum_{\sigma= \pm 1}\left|\widetilde{\chi^{\sigma}}\right|^{2} \delta\left(-p^{\prime}\right) \delta_{l^{\prime}} \delta_{m m^{\prime}} \\
& \left.\equiv P(p<<1, \alpha, t) \delta_{-}-p\right) \delta_{l l^{\prime}} \delta_{m m^{\prime}},
\end{aligned}
$$

where the amplit a the normalized power spectrum of axion at long wave leng imit is defined as:

$$
\begin{aligned}
\mathcal{P}(p<<1, \mathrm{v} & =\frac{p^{3}}{2 \pi^{2}} P\left(p<<1, \alpha, t_{\mathbf{L}}\right) \\
= & P_{\mathbf{B D}}\left(p, t_{\mathbf{L}}\right)(\cosh 2 \alpha-\sinh 2 \alpha) \\
= & \exp (-2 \alpha) P_{\mathbf{B D}}\left(p<<1, t_{\mathbf{L}}\right),
\end{aligned}
$$

with $P_{\mathbf{B D}}\left(p<<1, t_{\mathbf{L}}\right)$ as defined earlier.

In the super horizon time scales $\left(t_{\mathbf{L}}>>1\right)$ of region $\mathbf{L}$ th amplitude of the normalized power spectrum of axion a the long wave length approximation from $\alpha$ vacua can be expressed as:

$$
\begin{aligned}
& \mathcal{P}\left(p<<1, \alpha, t_{\mathbf{L}}>>1\right) \\
& \quad=\mathcal{P}_{\mathbf{B D}}\left(p<<1, t_{\mathbf{L}}>>1\right)(\cosh 2 \alpha-\sinh 2 \alpha) \\
& \quad=\exp (-2 \alpha) \mathcal{P}_{\mathbf{B D}}\left(p<<1, t_{\mathbf{L}}>>1\right),
\end{aligned}
$$

where $\mathcal{P}_{\mathbf{B D}}\left(p<<1, t_{\mathbf{L}}>>1\right)$ is defined in Eq. (3.35). It may be noted that, for $\alpha=0$ we get back the results obtained for Bunch Davies vacuum.

In Fig. 7a-c we have shown the behaviour of the power spectrum of the mean square vacuum fluctuation computed from FOE formalism in the small wave number regime. The values of $\alpha$ and the values of the mass parameter $v$ used here are same as those taken for large wave number regime. As expected, the behaviour for the the two limiting cases are distinct. However, the characteristics observed for $\alpha$ and $v$ dependences for both the cases are almost similar.

\subsection{Quantum vacuum fluctuation using reduced density matrix (RDM) formalism (with mixed state)}

In this section, we study the features of the two point correlation function of the quantum vacuum fluctuations and the associated primordial power spectrum using the reduced density matrix formalism. In Fig. 8 we have presented a schematic diagram for the computation algorithm of reduced 


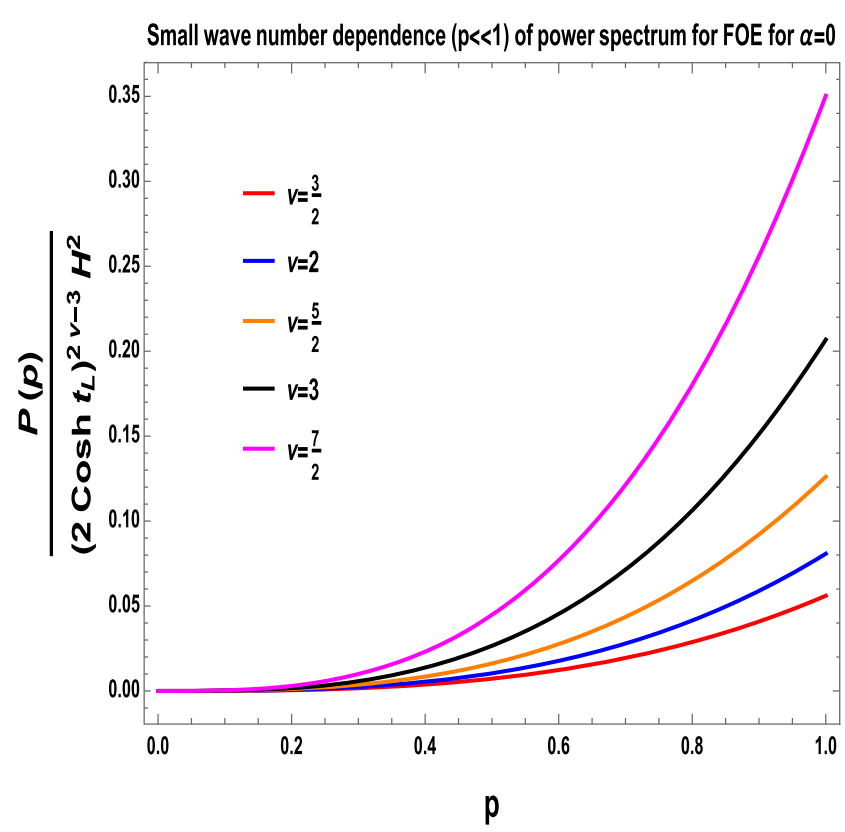

(a) Small wave number dependence of FOE power spectrum for $\alpha=0$

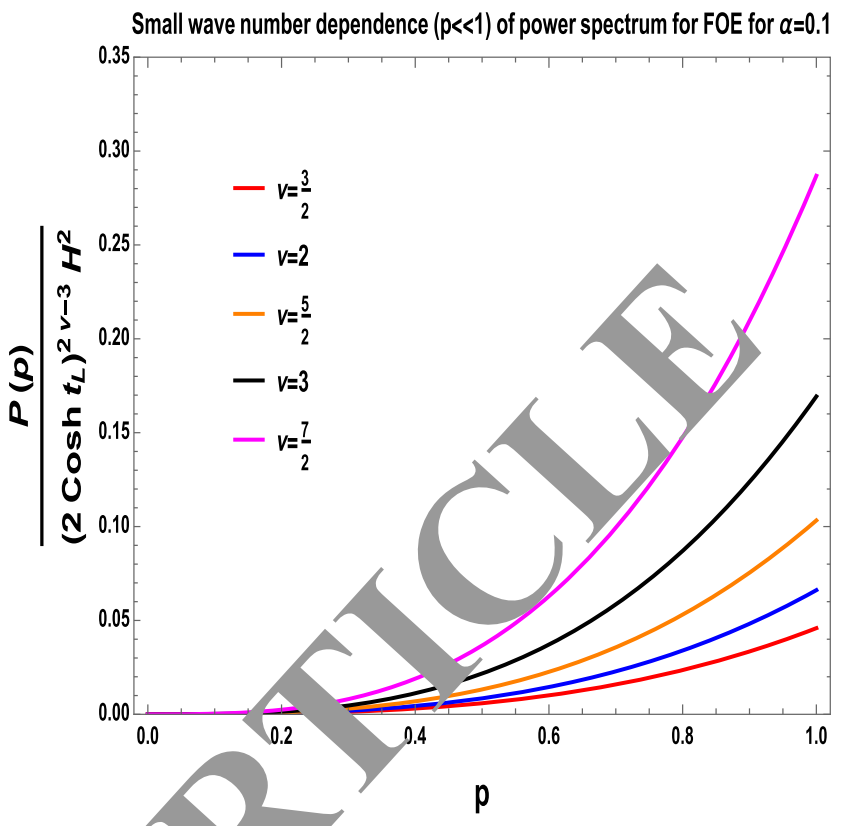

(b) Sn. wave number dependence of FOE power spectrum for $y=0.1$.

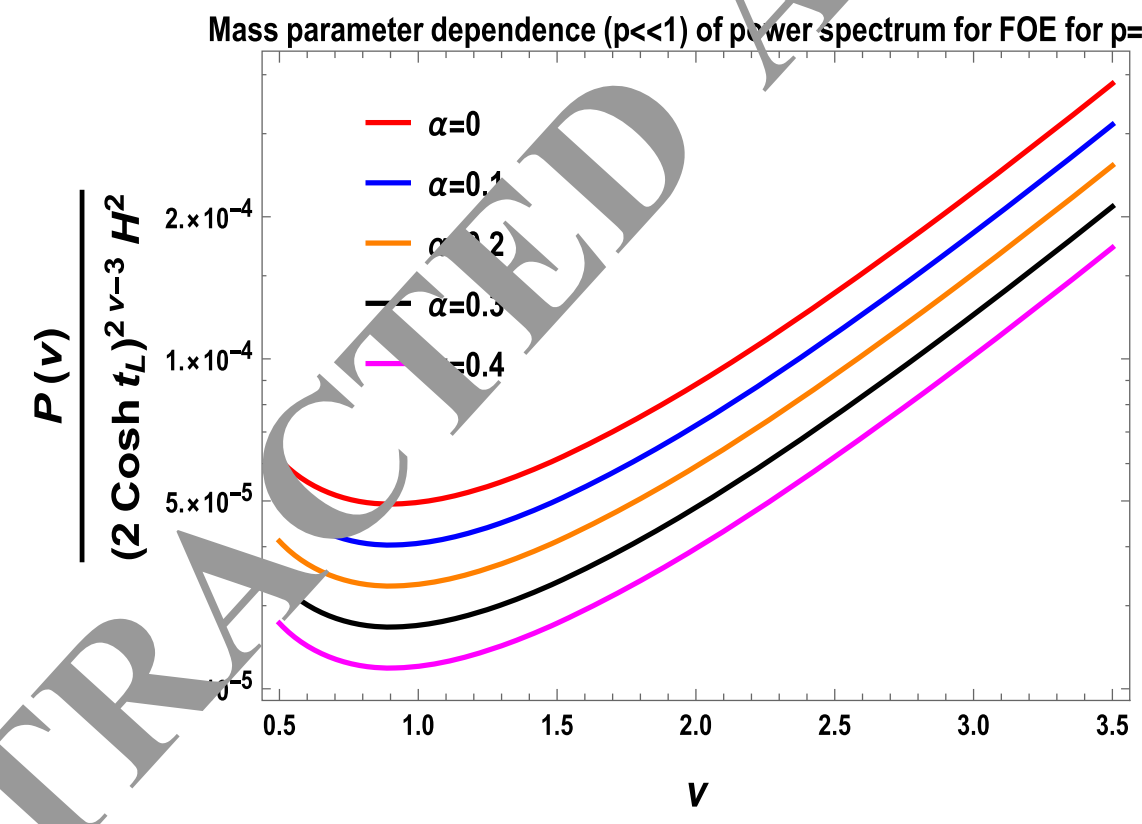

(c) Mass parameter dependence of FOE power spectrum for $p<<1$.

Fig. 7 Featu. of $F$ E E power spectrum in small wave number region

dens matrix formalism for mixed quantum state of axion in de S, ter hyperbolic open chart.

\subsubsection{Reduced density matrix (RDM) formalism}

We first write down the Fourier mode of the field operator, which is also the total solution of the field equation for axion in presence of source contribution. We start directly from the solution obtained in Eq. (2.20) and rewrite it in terms of the following matrix equation:

$\chi^{I}=\frac{1}{\mathcal{N}_{p}} \mathcal{M}_{J}^{I} \mathcal{P}^{J}+\sum_{n=0}^{\infty} \frac{1}{\mathcal{N}_{p,(n)}}\left(\mathcal{M}_{(n)}\right)_{J}^{I} \mathcal{P}_{(n)}^{J}$ 


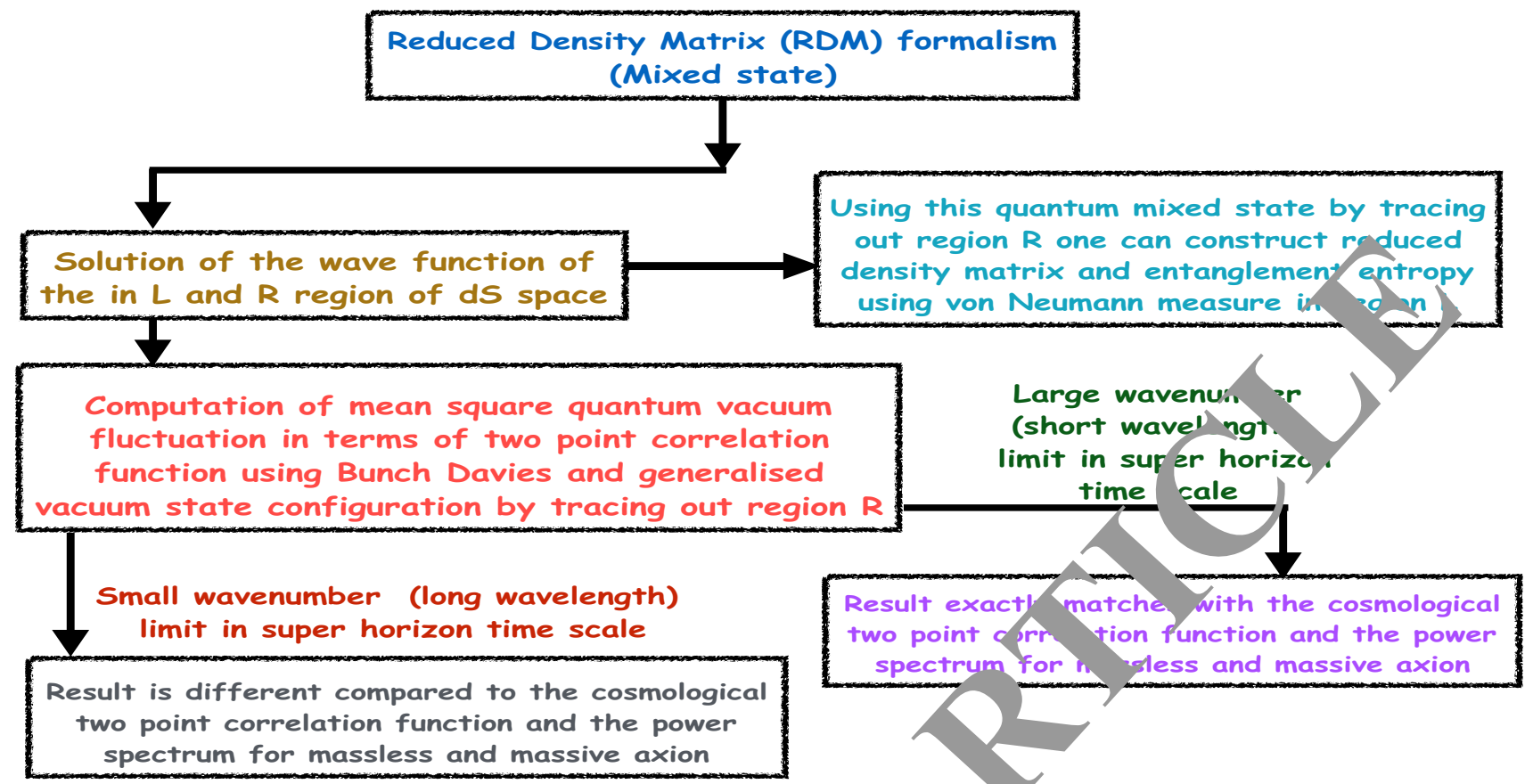

Fig. 8 Schematic diagram for the computation algorithm of reduced density ma for mixed quantum state of axion in de Sitter hyperbolic open chart

where for the complementary part of the solution we ha defined the following matrices:

$$
\begin{aligned}
\mathcal{M}_{J}^{I} & =\left(\begin{array}{cc}
\alpha_{q}^{\sigma} & \beta_{q^{\sigma}}^{\sigma} \\
\beta_{q}^{\sigma^{*}} & \alpha_{q}^{\sigma^{*}}
\end{array}\right), \quad \chi^{I}=\left(\begin{array}{c}
\chi_{\sigma}(t) \\
\chi_{\sigma}^{*}(t),
\end{array}\right), \\
\mathcal{P}^{J} & =\left(\begin{array}{c}
\mathcal{P}^{q} \\
\mathcal{P}^{q^{*}},
\end{array}\right) .
\end{aligned}
$$

Similarly for the particular solution, $v$ e define the following matrices:

$$
\left(\mathcal{M}_{(n)}\right)_{J}^{I}=\left(\begin{array}{cc}
\bar{\alpha}_{q, n}^{\sigma} & \bar{\beta}_{q, n}^{\sigma} \\
\bar{\beta}_{q, n}^{\sigma *} & \bar{\alpha}_{q, n}^{\sigma^{*}}
\end{array}\right), \quad\left(\begin{array}{c}
\mathcal{P}^{q, n} \\
\mathcal{P}^{q^{*}, n}
\end{array}\right),
$$

where $\sigma= \pm 1, q=\mathbf{R} \mathbf{L}$ a $\mathrm{d} I, J \leqslant 1,2,3,4$.

The redefined norn 7 an constant for the particular part of the soluti on $\mathcal{N}_{p,(n,}$ n be expressed as, $\mathcal{N}_{p,(n)}=$ $2 \sinh \pi p_{n} \sqrt{\mathcal{N} p_{n} \mathrm{O}}\left(n^{2}-p_{n}^{2}\right)$. Further using Eq. (3.40) the Bunch-Dav's mode ction can be written as:

$$
\frac{H}{\sin b} \widehat{a_{I} \chi}{ }^{I_{I}}\left[\frac{1}{\mathcal{N}_{p}} \mathcal{M}_{J}^{I} \mathcal{P}^{J}+\sum_{n=0}^{\infty} \frac{1}{\mathcal{N}_{p,(n)}}\left(\mathcal{M}_{(n)}\right)_{J}^{I} \mathcal{P}_{(n)}^{J}\right] \text {, }
$$

where $a_{I}=\left(a_{\sigma}, a_{\sigma}^{\dagger}\right)$ represents a set of creation and annihilation operators.

We also define the following operators:

$$
b_{J}=a_{I}^{(c)} \mathcal{M}_{J}^{I}, \quad b_{J(n)}=a_{I(n)}^{(p)}\left(\mathcal{M}_{(n)}\right)_{J}^{I},
$$

ere $a_{I}^{(c)}=\left(a_{\sigma}^{(c)}, a_{\sigma}^{(c) \dagger}\right)$ and $a_{I(n)}^{(p)}=\left(a_{\sigma, n}^{(p)}, a_{\sigma, n}^{(p) \dagger}\right)$ are the se of creation and annihilation operators which act on the omplementary and particular part respectively. Thus, the operator contribution for the total solution is:

$a_{I}=\left[a_{I}^{(c)}+\sum_{n=0}^{\infty} a_{I(n)}^{(p)}\right]$,

where by inverting Eq. (3.44) we have expressed:

$a_{I}^{(c)}=b_{J}\left(\mathcal{M}^{-1}\right)_{J}^{I}, \quad a_{I(n)}^{(p)}=b_{J(n)}\left(\mathcal{M}_{(n)}^{-1}\right)_{J}^{I}$.

The inverse matrices are defined as:

$\left(\mathcal{M}^{-1}\right)_{J}^{I}=\left(\begin{array}{ll}\gamma_{\sigma q} & \delta_{\sigma q} \\ \delta_{\sigma q}^{*} & \gamma_{\sigma q}^{*}\end{array}\right),\left(\mathcal{M}_{(n)}^{-1}\right)_{J}^{I}=\left(\begin{array}{ll}\bar{\gamma}_{\sigma q, n} & \bar{\delta}_{\sigma q, n} \\ \bar{\delta}_{\sigma q, n}^{*} & \bar{\gamma}_{\sigma q, n}^{*}\end{array}\right)$,

where $\sigma= \pm 1, q=\mathbf{R}, \mathbf{L}$ and $I, J=1,2,3,4$.

For further computation, $\alpha$-vacua are defined in terms of Bunch Davies vacuum state as:

$|\alpha\rangle=\exp \left(\frac{1}{2} \tanh \alpha \sum_{\sigma= \pm 1} a_{\sigma}^{\dagger} a_{\sigma}\right)|\mathbf{B D}\rangle$.

It is to be noted that for $\alpha=0$ we get, $|\alpha=0\rangle=|0\rangle=|\mathbf{B D}\rangle$. Moreover, we can also write the $\mathbf{R}$ and $\mathbf{L}$ vacua as:

$$
|\mathbf{R}\rangle=|\mathbf{R}\rangle_{(c)}+\sum_{n=0}^{\infty}|\mathbf{R}\rangle_{(p), n},
$$




$$
|\mathbf{L}\rangle=|\mathbf{L}\rangle_{(c)}+\sum_{n=0}^{\infty}|\mathbf{L}\rangle_{(p), n},
$$

with subscripts $(c)$ and $(p)$ representing the complementary and particular part respectively.

Further assuming the bipartite Hilbert space $\left(\mathcal{H}_{\alpha}:=\mathcal{H}_{\mathbf{R}} \otimes\right.$ $\mathcal{H}_{\mathbf{L}}$ ) one can also write the $\alpha$-vacua in terms of the $\mathbf{R}$ and $\mathbf{L}$ vacuum as:

$$
\begin{aligned}
|\alpha\rangle= & \exp \left(\frac{1}{2} \tanh \alpha \sum_{\sigma= \pm 1} a_{\sigma}^{\dagger} a_{\sigma}\right) \\
& \underbrace{\exp \left(\frac{1}{2} \sum_{i, j=\mathbf{R}, \mathbf{L}} m_{i j} b_{i}^{\dagger} b_{j}^{\dagger}+\frac{1}{2} \sum_{i, j=\mathbf{R}, \mathbf{L} n=0} \sum_{i}^{\infty} \bar{m}_{i j, n} \bar{b}_{i, n}^{\dagger} \bar{b}_{j, n}^{\dagger}\right)(|\mathbf{R}\rangle \otimes|\mathbf{L}\rangle)}_{\text {Bunch-Davies contribution }},
\end{aligned}
$$

where the matrices $m_{i j}$ and $\bar{m}_{i j, n}$ are defined for the complementary and particular part of the solution obtained for Bunch Davies vacuum state. In other words by setting $\alpha=0$ we get the following expression for the Bunch Davies quantum state:

$$
\begin{aligned}
|\mathbf{B D}\rangle= & \exp \left(\frac{1}{2} \sum_{i, j=\mathbf{R}, \mathbf{L}} m_{i j} b_{i}^{\dagger} b_{j}^{\dagger}+\frac{1}{2} \sum_{i, j=\mathbf{R}, \mathbf{L}} \sum_{n=0}^{\infty} \bar{m}_{i j, n} \bar{b}_{i, n}^{\dagger} \bar{b}_{j, n}^{\dagger}\right) \\
& \times(|\mathbf{R}\rangle \otimes|\mathbf{L}\rangle) .
\end{aligned}
$$

Also the creation and annihilation operators for $\tau_{\mathrm{L}} \mathrm{P}$ and $\mathbf{L}$ vacuum are defined in terms of new $b$ type of oscit using Bogoliubov transformation as:

$$
\begin{aligned}
a_{\sigma}= & \sum_{q=\mathbf{R}, \mathbf{L}}\left\{\left[\gamma_{q \sigma} b_{q}+\delta_{q \sigma}^{*} b_{q}^{\dagger}\right]+\sum_{n=0}^{\infty}\left[\left(\sigma_{\sigma, n} \bar{b}_{q, n}+\bar{\delta}_{q \sigma, n}^{*} \bar{b}_{q, n}^{\dagger}\right]\right\}\right. \\
& \forall \sigma= \pm 1, \\
a_{\sigma}^{\dagger}= & \sum_{q=\mathbf{R}, \mathbf{L}}\left\{\left[\gamma_{q \sigma}^{*} b_{q}^{\dagger}+\delta\right]+\sum_{n=0}^{\infty}\left[\bar{\gamma}_{q \sigma, n}^{*} \bar{b}_{q, n}^{\dagger}+\bar{\delta}_{q \sigma, n} \bar{b}_{q, n}\right]\right\} \\
& \forall \sigma= \pm 1 .
\end{aligned}
$$

Here $\gamma_{q \sigma}, \delta_{q \sigma}, \bar{\gamma}_{q \sigma,}$, and $\bar{\delta}_{q \sigma, n}$ are the coefficient matrices. For ur further computation we use the definition of $\alpha$-vacuur a (a) d Bunch Davies vacuum state), which is very ful anpute long range cosmological correlation f. ctio s in de Sitter space. In the context of $\alpha$-vacua the cre. $n$ and annihilation operators are defined in terms of the cor atuents of $\mathbf{R}$ or $\mathbf{L}$ vacuum state as:

$$
\begin{aligned}
d_{\sigma}= & \sum_{q=\mathbf{R}, \mathbf{L}}\left\{\left[\left(\cosh \alpha \gamma_{q \sigma}-\sinh \alpha \delta_{q \sigma}\right) b_{q}\right.\right. \\
& \left.+\left(\cosh \alpha \delta_{q \sigma}^{*}-\sinh \alpha \gamma_{q \sigma}^{*}\right) b_{q}^{\dagger}\right]
\end{aligned}
$$

$$
\begin{aligned}
+ & {\left[\left(\cosh \alpha \sum_{n=0}^{\infty} \bar{\gamma}_{q \sigma, n} \bar{b}_{q, n}-\sinh \alpha \sum_{n=0}^{\infty} \bar{\delta}_{q \sigma, n} \bar{b}_{q, n}\right)\right.} \\
+ & \left.\left.\left(\cosh \alpha \sum_{n=0}^{\infty} \bar{\delta}_{q \sigma, n}^{*} \bar{b}_{q, n}^{\dagger}-\sinh \alpha \sum_{n=0}^{\infty} \bar{\gamma}_{q \sigma, n}^{*} \bar{b}_{q, n}^{\dagger}\right)\right]\right\} \\
& \forall \sigma= \pm 1, \\
d_{\sigma}^{\dagger}= & \sum_{q=\mathbf{R}, \mathbf{L}}\left\{\left[\left(\cosh \alpha \gamma_{q \sigma}^{*}-\sinh \alpha \delta_{q \sigma}^{*}\right)\right.\right. \\
+ & \left(\cosh \alpha \delta_{q \sigma}-\sinh \alpha \gamma_{q \sigma}\right) b_{q} \\
+ & {\left[\left(\cosh \alpha \sum_{n=0}^{\infty} \bar{\gamma}_{q \sigma, n}^{*} \bar{b}\right.\right.} \\
+ & \left.\left.\left.\left(\cosh \alpha \sum_{i=0}^{\infty} \delta_{q \sigma, n} \bar{b}_{q, n}\right) \sinh \alpha \sum_{n=0}^{\infty} \bar{\gamma}_{q \sigma, n} \bar{b}_{q, n}\right)\right]\right\} \\
& \forall \sigma=+1
\end{aligned}
$$

where we use definition of creation and annihilation operators Runch b,avies vacuum as mentioned in Eqs. (3.53) and $(3.52 y$ his computation it is important to note that, under B goliubov transformation the original matrix $\gamma_{q \sigma}$, $\delta_{q \sigma}, \bar{\gamma}_{q \sigma, h}$ and $\bar{\delta}_{q \sigma, n}$ used for Bunch Davies vacuum trans$\mathrm{m}$ ( for $\alpha$-vacua) as:

$$
\begin{aligned}
& \gamma_{q \sigma} \longrightarrow\left(\cosh \alpha \gamma_{q \sigma}-\sinh \alpha \delta_{q \sigma}\right), \\
& \delta_{q \sigma} \longrightarrow\left(\cosh \alpha \delta_{q \sigma}-\sinh \alpha \gamma_{q \sigma}\right) \\
& \bar{\gamma}_{q \sigma, n} \longrightarrow\left(\cosh \alpha \bar{\gamma}_{q \sigma, n}-\sinh \alpha \bar{\delta}_{q \sigma, n}\right) \\
& \bar{\delta}_{q \sigma, n} \longrightarrow\left(\cosh \alpha \bar{\delta}_{q \sigma, n}-\sinh \alpha \bar{\gamma}_{q \sigma, n}\right)
\end{aligned}
$$

Thus, after the Bogoliubov transformation, $\alpha$-vacua state can be written in terms of $\mathbf{R}$ and $\mathbf{L}$ vacua as:

$$
\begin{aligned}
|\alpha\rangle= & \exp \left(\frac{1}{2} \sum_{i, j=\mathbf{R}, \mathbf{L}} \tilde{m}_{i j} b_{i}^{\dagger} b_{j}^{\dagger}+\frac{1}{2} \sum_{i, j=\mathbf{R}, \mathbf{L}} \sum_{n=0}^{\infty} \tilde{\tilde{m}}_{i j, n} \bar{b}_{i, n}^{\dagger} \bar{b}_{j, n}^{\dagger}\right) \\
& \times(|\mathbf{R}\rangle \otimes|\mathbf{L}\rangle),
\end{aligned}
$$

Here $\tilde{m}_{i j}$ and $\overline{\tilde{m}}_{i j, n}$ represent the entries of the matrices corresponding to the complementary and particular solution respectively and we will compute them by demanding $d_{\sigma}|\alpha\rangle=0$, and keeping only linear terms of creation operators. This directly yields the following:

$$
\begin{aligned}
& {\left[\tilde{m}_{i j}\left(\cosh \alpha \gamma_{j \sigma}-\sinh \alpha \delta_{j \sigma}\right)\right.} \\
& \left.\quad+\left(\cosh \alpha \delta_{i \sigma}^{*}-\sinh \alpha \gamma_{i \sigma}^{*}\right)\right]=0, \\
& \quad\left[\left(\cosh \alpha \overline{\tilde{m}}_{i j, n} \bar{\gamma}_{j \sigma, n}-\sinh \alpha \bar{m}_{i j, n} \bar{\delta}_{j \sigma, n}\right)\right. \\
& \left.\quad+\left(\cosh \alpha \bar{\delta}_{i \sigma, n}^{*}-\sinh \alpha \bar{\gamma}_{i \sigma, n}^{*}\right)\right]=0 \forall n .
\end{aligned}
$$


From these two equations, the matrices corresponding to the complementary and particular part of the solution can be expressed as:

$$
\begin{aligned}
\tilde{m}_{i j}= & -\left(\cosh \alpha \delta_{i \sigma}^{*}-\sinh \alpha \gamma_{i \sigma}^{*}\right) \\
& \times(\cosh \alpha \gamma-\sinh \alpha \delta)_{\sigma j}^{-1}=\left(\begin{array}{cc}
\tilde{m}_{\mathbf{R R}} & \tilde{m}_{\mathbf{R L}} \\
\tilde{m}_{\mathbf{L R}} & \tilde{m}_{\mathbf{L L}}
\end{array}\right), \\
\overline{\tilde{m}}_{i j, n}= & -\left(\cosh \alpha \bar{\delta}_{i \sigma, n}^{*}-\sinh \alpha \bar{\gamma}_{i \sigma, n}^{*}\right) \\
& \times(\cosh \alpha \bar{\gamma}-\sinh \alpha \bar{\delta})_{\sigma j, n}^{-1}=\left(\begin{array}{ll}
\bar{m}_{\mathbf{R R}, n} & \bar{m}_{\mathbf{R L}, n} \\
\bar{m}_{\mathbf{L R}, n} & \bar{m}_{\mathbf{L L}, n}
\end{array}\right) .
\end{aligned}
$$

Substituting the expressions for $\gamma, \delta, \gamma_{n}$ and $\delta_{n}$ we finally obtain the entries of the mass matrices for $i, j=\mathbf{R}, \mathbf{L}$ as:

$\tilde{m}_{i j}=e^{i \theta} \frac{\sqrt{2} e^{-p \pi} \mathcal{T}_{i j}^{(\nu)}}{\sqrt{\cosh 2 \pi p+\cos 2 \pi \nu}\left(\cosh ^{2} \alpha+\sinh ^{2} \alpha e^{-2 \pi(p+i v)}\right)}$

$\overline{\tilde{m}}_{i j, n}=e^{i \theta} \frac{\sqrt{2} e^{-p_{n} \pi} \mathcal{T}_{i j}^{(v, n)}}{\sqrt{\cosh 2 \pi p_{n}+\cos 2 \pi v}\left(\cosh ^{2} \alpha+\sinh ^{2} \alpha e^{-2 \pi\left(p_{n}+i v\right)}\right)}$

where we defined the $\mathcal{T}$ matrices as:

$$
\begin{aligned}
\mathcal{T}_{i j}^{(v)} & =\left(\begin{array}{ll}
\mathcal{T}_{\mathbf{R R}}^{(v)} & \mathcal{T}_{\mathbf{R L}}^{(v)} \\
\mathcal{T}_{\mathbf{L R}}^{(v)} & \mathcal{T}_{\mathbf{L L}}^{(v)}
\end{array}\right), \\
\mathcal{T}_{i j}^{(v, n)} & =\left(\begin{array}{ll}
\mathcal{T}_{\mathbf{R R}}^{(v, n)} & \mathcal{T}_{\mathbf{R L}}^{(v, n)} \\
\mathcal{T}_{\mathbf{L R}}^{(v, n)} & \mathcal{T}_{\mathbf{L L}}^{(v, n)}
\end{array}\right) .
\end{aligned}
$$

and the corresponding entries of the 2 matrices are given by:

$$
\begin{aligned}
& \mathcal{T}_{\mathbf{R R}}^{(v)}=\mathcal{T}_{\mathbf{L} \mathbf{L}}^{(v)}=\left(\cosh ^{2} \alpha,-\sinh ^{2} \alpha e^{-2 i \pi v}\right) \\
& \left.\sinh 2 \alpha \operatorname{si}_{1} \Lambda^{2} \pi p e^{-i \pi v} \sec \pi v\right] \cos \pi v \\
& \mathcal{T}_{\mathbf{R L}}^{(v)}={ }^{\prime}=i\left[\cosh ^{2} \alpha+\sinh ^{2} \alpha e^{-2 i \pi v}\right. \\
& \left.\perp \perp \sinh 2 \alpha \cos \pi \nu e^{-i \pi \nu}\right] \sinh \pi p, \\
& \mathcal{T}_{\mathbf{R R}}^{\left(v, h_{h}\right.}-\mathcal{T}_{\mathbf{L L}}^{(v, n)}=\left[\left(\cosh ^{2} \alpha+\sinh ^{2} \alpha e^{-2 i \pi v}\right)\right. \\
& \left.-\sinh 2 \alpha \sinh ^{2} \pi p_{n} e^{-i \pi v} \sec \pi v\right] \cos \pi v, \\
& \mathcal{T}_{\mathbf{R L}}^{(v, n)}=\mathcal{T}_{\mathbf{L R}}^{(v, n)}=i\left[\cosh ^{2} \alpha+\sinh ^{2} \alpha e^{-2 i \pi v}\right. \\
& \left.+\sinh 2 \alpha \cos \pi v e^{-i \pi v}\right] \sinh \pi p_{n} .
\end{aligned}
$$

For the massless $(v=3 / 2)$ axion case, we obtain the following simplified expressions:

$\tilde{m}_{i j}=e^{i \theta} \frac{\sqrt{2} e^{-p \pi} \mathcal{T}_{i j}^{(3 / 2)}}{\sqrt{\cosh 2 \pi p-1}\left(\cosh ^{2} \alpha-\sinh ^{2} \alpha e^{-2 \pi p}\right)}$

$\overline{\tilde{m}}_{i j, n}=e^{i \theta} \frac{\sqrt{2} e^{-p_{n} \pi} \mathcal{T}_{i j}^{(3 / 2, n}}{\sqrt{\cosh 2 \pi p_{n}-1}\left(\cosh ^{2} v-\sinh ^{2} \alpha e^{\left.-2 \pi p_{n}\right)}\right.}$

where we have defined th $\mathcal{T}\left(\mathrm{mat}^{\prime}\right.$ ices as:

$$
\begin{aligned}
\mathcal{T}_{i j}^{(3 / 2)} & =\left(\begin{array}{ll}
\mathcal{T}_{\mathbf{R}}^{(3 / 2)} & -(3 / 2) \\
\mathcal{T}^{(3 / 2)} & \left.\mathcal{T}_{\mathbf{L} /} / 2\right)
\end{array}\right), \\
\mathcal{T}_{i j}^{(3 / 2, n)} & =\left(\begin{array}{ll}
\mathcal{T}_{\mathbf{R L}}^{(3 / 2, n)} \\
\mathcal{T}_{\mathbf{L} \mathbf{l}}^{\left(\mathbf{R}^{\prime}(2, n)\right.} & \mathcal{T}_{\mathbf{L} \mathbf{L}}^{(3 / 2, n)}
\end{array}\right) .
\end{aligned}
$$

and the cc responding entries of the $\mathcal{T}^{(3 / 2)}$ matrices are given

$$
\begin{aligned}
\mathcal{T}_{\mathbf{R}}^{(3 / 2)} & =\mathcal{T}_{\mathbf{L} \mathbf{L}}^{(3 / 2)}=0, \\
\mathcal{T}_{\mathbf{R L}}^{(3 / 2)} & =\mathcal{T}_{\mathbf{L R}}^{(3 / 2)}=i \sinh \pi p, \\
\mathcal{T}_{\mathbf{R R}}^{(3 / 2, n)} & =\mathcal{T}_{\mathbf{L L}}^{(3 / 2, n)}=0, \\
\mathcal{T}_{\mathbf{R} \mathbf{L}}^{(3 / 2, n)} & =\mathcal{T}_{\mathbf{L R}}^{(3 / 2, n)}=i \sinh \pi p_{n} .
\end{aligned}
$$

In the above analysis, we have considered small axion mass $\left(v^{2}>0\right)$ limiting situations with an arbitrary parameter $\alpha$, which corresponds to Bunch Davies vacuum state with the choice $\alpha=0$. For completeness, we also consider the large axion mass $\left(v^{2}<0\right.$ where $\left.v \rightarrow-i|v|\right)$ limiting situation which is very important to study the imprints of quantum entanglement in cosmological correlation functions. In this large axion mass limiting situation, we actually consider a specific window of $\mathbf{S O}(\mathbf{1}, \mathbf{3})$ principal quantum number, which is bounded within the range $0<p<|\nu|$. Consequently, the entries of the coefficient matrix $\tilde{m}$ can be approximated as:

$$
\begin{aligned}
& \tilde{m}_{\mathbf{R R}}=-\sqrt{\frac{\cosh (|\nu|-p)}{\cosh (|\nu|+p)}} \\
& \times \frac{2\left[\cosh 2 \alpha \cosh ^{2} \pi|\nu|-\sinh 2 \alpha \sinh ^{2} \pi p+\frac{1}{2} \sinh 2 \pi|\nu|\right]}{\left(e^{2 \pi p}+e^{2 \pi|\nu|}\right) \cosh ^{2} \alpha+\left(e^{2 \pi p}+e^{2 \pi|v|}\right) \sinh ^{2} \alpha},
\end{aligned}
$$




$$
\begin{aligned}
& \tilde{m}_{\mathbf{R L}}=-\sqrt{\frac{\cosh (|v|-p)}{\cosh (|\nu|+p)}} \\
& \times \frac{2 i[(\cosh 2 \alpha+\sinh 2 \alpha) \cosh \pi|\nu|+\sinh \pi|\nu|]}{\left(e^{2 \pi p}+e^{2 \pi|v|}\right) \cosh ^{2} \alpha+\left(e^{2 \pi p}+e^{2 \pi|v|}\right) \sinh ^{2} \alpha},
\end{aligned}
$$

which for $\alpha=0$ yield a simplified expression for the $\tilde{m}$ with Bunch Davies vacuum state. We note that for general value of $\alpha$ and for large axion mass $\left(v^{2}<0\right.$ where $\left.v \rightarrow-i|v|\right)$, we always get real value for $\tilde{m}_{\mathbf{R R}}$ and imaginary value for $\tilde{m}_{\mathbf{R} \mathbf{L}}$. This is an important observation for our further analysis.

From the perspective of cosmological observation in the superhorizon time scale, we again consider two further limiting situations: (a) large wave number $(p>>1)$ or small wave length limit and (b)small wave number $(p<<1)$ or large wave length limit.

Using these two limiting situations we can simplify the expression for the entries of the coefficient matrix $\tilde{m}$ considering both small and large axion mass. We start with the expressions for small axion mass limit in large wave number $(p>>1)$ approximation:

$$
\begin{aligned}
& \tilde{m}_{i j} \approx 2 e^{i \theta} e^{-2 p \pi} \widetilde{\mathcal{T}}_{i j}^{(v)} \operatorname{sech}^{2} \alpha \\
& \overline{\tilde{m}}_{i j, n} \approx 2 e^{i \theta} e^{-2 p_{n} \pi} \widetilde{\mathcal{T}}_{i j}^{(v, n)} \operatorname{sech}^{2} \alpha
\end{aligned}
$$

where we have defined the $\tilde{\mathcal{T}}$ matrices for $p>>1$ limit a

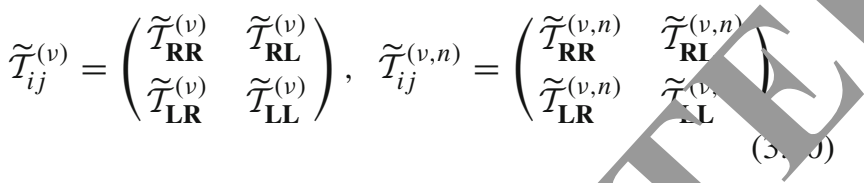

and the corresponding entries of the $\tilde{\mathcal{T}}$ natrices for $\rho>>1$ limit are given by the following simp fied expressions:

$$
\begin{aligned}
& \widetilde{\mathcal{T}}_{\mathbf{R R}}^{(v)}=\widetilde{\mathcal{T}}_{\mathbf{L L}}^{(v)}=\left[\left(\cosh ^{2} \alpha+\sinh ^{2} \alpha e-v\right)\right. \\
& \left.-\frac{1}{4} \sinh 2 \alpha e^{2 n-i \pi \nu} \sec \pi v\right] \cos \pi \nu, \\
& \tilde{\mathcal{T}}_{\mathbf{R L}}^{(v)}=\mathcal{T}_{\mathbf{L R}}^{(v)}=i_{L}^{\cosh }+\sinh ^{2} \alpha e^{-2 i \pi v} \\
& \left.+\sinh 2 a \text { s } \pi v e^{-i \pi \nu}\right] \frac{1}{2} e^{\pi p}, \\
& \tilde{\mathcal{T}}_{\mathbf{R R}}^{(v, n)}=\tau_{\mathbf{T}}^{(\hat{V} n)}=\left[\left(\cosh ^{2} \alpha+\sinh ^{2} \alpha e^{-2 i \pi v}\right)\right. \\
& \left.-\frac{1}{4} \operatorname{lnh} 2 \alpha e^{2 p_{n} \pi} e^{-i \pi v} \sec \pi \nu\right] \cos \pi \nu, \\
& \widetilde{\mathcal{T}}_{\mathbf{R L}}^{(v, n)} \widetilde{\mathcal{I}}_{\mathbf{L R}}^{(v, n)}=i\left[\cosh ^{2} \alpha+\sinh ^{2} \alpha e^{-2 i \pi v}\right. \\
& \left.+\sinh 2 \alpha \cos \pi v e^{-i \pi v}\right] \frac{1}{2} e^{\pi p_{n}} .
\end{aligned}
$$

For massless $(v=3 / 2)$ axion, we get the following simplified expressions:

$$
\tilde{m}_{i j} \approx 2 e^{i \theta} e^{-2 p \pi} \widetilde{\mathcal{T}}_{i j}^{(3 / 2)} \operatorname{sech}^{2} \alpha
$$

$$
\overline{\tilde{m}}_{i j, n} \approx 2 e^{i \theta} e^{-2 p_{n} \pi} \widetilde{\mathcal{T}}_{i j}^{(3 / 2, n)} \operatorname{sech}^{2} \alpha
$$

where the $\tilde{\mathcal{T}}^{(3 / 2)}$ matrices (for $p>>1$ ) are given by:

$$
\begin{aligned}
\tilde{\mathcal{T}}_{i j}^{(3 / 2)} & =\left(\begin{array}{ll}
\tilde{\mathcal{T}}_{\mathbf{R}}^{(3 / 2)} & \widetilde{\mathcal{T}}_{\mathbf{R}}^{(3 / 2)} \\
\widetilde{\mathcal{T}}_{\mathbf{L R}}^{(3 / 2)} & \widetilde{\mathcal{T}}_{\mathbf{L} \mathbf{L}}^{(3 / 2)}
\end{array}\right), \\
\widetilde{\mathcal{T}}_{i j}^{(3 / 2, n)} & =\left(\begin{array}{ll}
\widetilde{\mathcal{T}}_{\mathbf{R}}^{(3 / 2, n)} & \widetilde{\mathcal{T}}_{\mathbf{R}}^{(3 / 2, n)} \\
\tilde{\mathcal{T}}_{\mathbf{L R}}^{(3 / 2, n)} & \widetilde{\mathcal{T}}_{\mathbf{L} \mathbf{L}}^{(3 / 2, n)}
\end{array}\right) .
\end{aligned}
$$

and the corresponding entries of $\tilde{\mathcal{T}}^{-}, \mathrm{m}$ arices are given by:

$$
\begin{aligned}
& \widetilde{\mathcal{T}}_{\mathbf{R R}}^{(3 / 2)}=\widetilde{\mathcal{T}}_{\mathbf{L} \mathbf{L}}^{(3 / 2)}=0, \\
& \tilde{\mathcal{T}}_{\mathbf{R} \mathbf{L}}^{(3 / 2)}=\mathcal{T}_{\mathbf{L R}}^{(3 / 2)}=\frac{l}{-} e^{\pi p}, \\
& \tilde{\mathcal{T}}_{\mathbf{R R}}^{(3 / 2, n)}=\tilde{\mathcal{T}}_{\mathbf{J}}^{(3 / 2, n)}=0 \\
& \tilde{\mathcal{T}}_{\mathbf{R L}}^{(3 / 2, n)}=\left(3 / 2, \frac{i}{2} e^{\pi p_{n}}\right. \text {. }
\end{aligned}
$$

On the ther han, , for small axion mass and for large wave number $(p, 1)$ we have:

$$
\approx e^{i \theta} \frac{\sqrt{2} e^{-p \pi} \hat{\mathcal{T}}_{i j}^{(v)}}{\sqrt{\cos 2 \pi v}\left(\cosh ^{2} \alpha+\sinh ^{2} \alpha e^{-2 \pi i v}\right)}
$$

where the $\hat{\mathcal{T}}$ matrices are defined as:

$$
\hat{\mathcal{T}}_{i j}^{(v)}=\left(\begin{array}{cc}
\hat{\mathcal{T}}_{\mathbf{R R}}^{(v)} & \hat{\mathcal{T}}_{\mathbf{R L}}^{(v)} \\
\hat{\mathcal{T}}_{\mathbf{L R}}^{(v)} & \hat{\mathcal{T}}_{\mathbf{L L}}^{(v)}
\end{array}\right), \quad \hat{\mathcal{T}}_{i j}^{(v, n)}=\left(\begin{array}{cc}
\hat{\mathcal{T}}_{\mathbf{R R}}^{(v, n)} & \hat{\mathcal{T}}_{\mathbf{R L}}^{(v, n)} \\
\hat{\mathcal{T}}_{\mathbf{L R}}^{(v, n)} & \hat{\mathcal{T}}_{\mathbf{L} \mathbf{L}}^{(v, n)}
\end{array}\right)
$$

and the corresponding entries of the $\hat{\mathcal{T}}$ matrices (for $p<<1$ ) are given by:

$$
\begin{aligned}
& \hat{\mathcal{T}}_{\mathbf{R R}}^{(v)}=\hat{\mathcal{T}}_{\mathbf{L L}}^{(v)} \\
& =\left[\left(\cosh ^{2} \alpha+\sinh ^{2} \alpha e^{-2 i \pi v}\right)\right. \\
& \left.-\sinh 2 \alpha \pi^{2} p^{2} e^{-i \pi \nu} \sec \pi \nu\right] \cos \pi \nu, \\
& \hat{\mathcal{T}}_{\mathbf{R L}}^{(v)}=\hat{\mathcal{T}}_{\mathbf{L R}}^{(v)} \\
& =i\left[\cosh ^{2} \alpha+\sinh ^{2} \alpha e^{-2 i \pi v}\right. \\
& \left.+\sinh 2 \alpha \cos \pi \nu e^{-i \pi \nu}\right] \pi p, \\
& \hat{\mathcal{T}}_{\mathbf{R R}}^{(v, n)}=\hat{\mathcal{T}}_{\mathbf{L L}}^{(v, n)} \\
& =\left[\left(\cosh ^{2} \alpha+\sinh ^{2} \alpha e^{-2 i \pi v}\right)\right. \\
& \left.-\sinh 2 \alpha \pi^{2} p_{n}^{2} e^{-i \pi v} \sec \pi v\right] \cos \pi \nu,
\end{aligned}
$$




$$
\begin{aligned}
\hat{\mathcal{T}}_{\mathbf{R L}}^{(v, n)}= & \hat{\mathcal{T}}_{\mathbf{L R}}^{(v, n)} \\
= & i\left[\cosh ^{2} \alpha+\sinh ^{2} \alpha e^{-2 i \pi v}\right. \\
& \left.+\sinh 2 \alpha \cos \pi v e^{-i \pi v}\right] \pi p_{n} .
\end{aligned}
$$

For the case of massless $(v=3 / 2)$ axion, we get the following simplified expressions:

$$
\begin{gathered}
\tilde{m}_{i j} \approx e^{i \theta} \sqrt{2} e^{-p \pi} \hat{\mathcal{T}}_{i j}^{(3 / 2)} \\
\overline{\tilde{m}}_{i j, n} \approx e^{i \theta} \sqrt{2} e^{-p_{n} \pi} \hat{\mathcal{T}}_{i j}^{(3 / 2, n)}
\end{gathered}
$$

with the $\hat{\mathcal{T}}$ matrices defined as:

$$
\begin{aligned}
\hat{\mathcal{T}}_{i j}^{(3 / 2)} & =\left(\begin{array}{ll}
\hat{\mathcal{T}}_{\mathbf{R R}}^{(3 / 2)} & \hat{\mathcal{T}}_{\mathbf{R L}}^{(3 / 2)} \\
\hat{\mathcal{T}}_{\mathbf{L R}}^{(3 / 2)} & \hat{\mathcal{T}}_{\mathbf{L} \mathbf{L}}^{(3 / 2)}
\end{array}\right), \\
\hat{\mathcal{T}}_{i j}^{(3 / 2, n)} & =\left(\begin{array}{ll}
\hat{\mathcal{T}}_{\mathbf{R R}}^{(3 / 2, n)} & \hat{\mathcal{T}}_{\mathbf{R} \mathbf{L}}^{(3 / 2, n)} \\
\hat{\mathcal{T}}_{\mathbf{L R}}^{(3 / 2, n)} & \hat{\mathcal{T}}_{\mathbf{L} \mathbf{L}}^{(3 / 2, n)}
\end{array}\right)
\end{aligned}
$$

$$
\begin{aligned}
|\alpha\rangle= & {\left[1-\left(\left|\gamma_{p}^{(\alpha)}\right|^{2}+\sum_{n=0}^{\infty}\left|\Gamma_{p, n}^{(\alpha)}\right|^{2}\right)\right]^{1 / 2} } \\
& \times \exp \left(\gamma_{p}^{(\alpha)} \tilde{c}_{\mathbf{R}}^{\dagger} \tilde{c}_{\mathbf{L}}^{\dagger}+\sum_{n=0}^{\infty} \Gamma_{p, n}^{(\alpha)} \tilde{C}_{\mathbf{R}, n}^{\dagger} \tilde{C}_{\mathbf{L}, n}^{\dagger}\right) \\
& \times\left(\left|\mathbf{R}^{\prime}\right\rangle \otimes\left|\mathbf{L}^{\prime}\right\rangle\right)^{(\alpha)},
\end{aligned}
$$

where $\gamma_{p}^{(\alpha)}$ and $\Gamma_{p, n}^{(\alpha)}$ are to be determined sh $1 \mathrm{v} /$ We note that the the relationship between the ew and theold basis is given by:

$$
\begin{aligned}
& (|\mathbf{R}\rangle \otimes|\mathbf{L}\rangle) \rightarrow\left(\left|\mathbf{R}^{\prime}\right\rangle \otimes\left|\mathbf{L}^{\prime}\right\rangle\right) \\
& =\left[1-\left(\left|\gamma_{p}^{(\alpha)}\right|^{2}+\sum_{-0}^{\infty} \mid \Gamma_{p, n}\right)\right] \\
& \times \exp \left(-\tilde{C}_{\mathbf{R}}^{\dagger} \tilde{c}_{\mathbf{L}}^{\dagger}-\sum_{n=0}^{\infty} \Gamma_{p, n}^{(\alpha)} \tilde{C}_{\mathbf{R}, n}^{\dagger} \tilde{C}_{\mathbf{L}, n}^{\dagger}\right) \\
& \times \sim \operatorname{~}\left(\frac{1}{2} \sum_{=\mathbf{R}, \mathbf{L}} m_{i j} b_{i}^{\dagger} b_{j}^{\dagger}+\frac{1}{2} \sum_{i, j=\mathbf{R}, \mathbf{L}} \sum_{n=0}^{\infty} \bar{m}_{i j, n} \bar{b}_{i, n}^{\dagger} \bar{b}_{j, n}^{\dagger}\right) \\
& \times(\mid \mathbf{K}\langle\otimes \mid \mathbf{L}\rangle) \text {. }
\end{aligned}
$$

and the corresponding entries of the $\hat{\mathcal{T}}^{(3 / 2)}$ matrices (fo commutation relations between the creation and annihi$p<<1$ ) are given by:

$$
\begin{aligned}
\hat{\mathcal{T}}_{\mathbf{R R}}^{(3 / 2)} & =\hat{\mathcal{T}}_{\mathbf{L}}^{(3 / 2)}=0, \\
\hat{\mathcal{T}}_{\mathbf{R L}}^{(3 / 2)} & =\hat{\mathcal{T}}_{\mathbf{L}}^{(3 / 2)}=i \pi p, \\
\hat{\mathcal{T}}_{\mathbf{R}}^{(3 / 2, n)} & =\hat{\mathcal{T}}_{\mathbf{L}}^{(3 / 2, n)}=0, \\
\hat{\mathcal{T}}_{\mathbf{R}}^{(3 / 2, n)} & =\hat{\mathcal{T}}_{\mathbf{L} \mathbf{R}}^{(3 / 2, n)}=i \pi p_{n} .
\end{aligned}
$$

For further analysis, it is converient to change over to a suitable basis by tracing over all po sio ntributions from $\mathbf{R}$ and $\mathbf{L}$ region. To achieve this we pe form another Bogoliubov transformation by intr fuci g new sets of operators:

$\tilde{c}_{\mathbf{R}}=\tilde{u} b_{\mathbf{R}}+\tilde{v} b_{\mathbf{K}} \tilde{c}_{\mathbf{L}}=\tilde{u} b_{\mathbf{L}}+\overline{\tilde{v}} b_{\mathbf{L}}^{\dagger}$,

$\left.\tilde{C}_{\mathbf{R}, n}=\tilde{U}_{h} b_{\mathbf{R}, n}+\tilde{V}_{n}\right\rangle_{\mathbf{R}, n}, \quad \tilde{C}_{\mathbf{L}, n}=\overline{\tilde{U}}_{n} b_{\mathbf{L}, n}+\overline{\tilde{V}}_{n} b_{\mathbf{L}, n}^{\dagger}$,

following conditions:

$$
\begin{aligned}
& |\tilde{u}|^{2}-|\tilde{v}|^{2}=1,|\overline{\tilde{u}}|^{2}-|\overline{\tilde{v}}|^{2}=1, \\
& \left|\tilde{U}_{n}\right|^{2}-\left|\tilde{V}_{n}\right|^{2}=1, \quad\left|\overline{\tilde{U}}_{n}\right|^{2}-\left|\overline{\tilde{V}}_{n}\right|^{2}=1 .
\end{aligned}
$$

Using these operators we write the $\alpha$-vacuum state in terms of new basis represented by the direct product of $\mathbf{R}^{\prime}$ and $\mathbf{L}^{\prime}$ vacuum state as: la on operators corresponding to the new sets of oscillators is taken as:

$$
\begin{aligned}
& {\left[\tilde{c}_{i}, \tilde{c}_{j}^{\dagger}\right]=\delta_{i j}, \quad\left[\tilde{c}_{i}, \tilde{c}_{j}\right]=0=\left[\tilde{c}_{i}^{\dagger}, \tilde{c}_{j}^{\dagger}\right],} \\
& {\left[\tilde{C}_{i, n}, \tilde{C}_{j, m}^{\dagger}\right]=\delta_{i j} \delta_{n m}, \quad\left[\tilde{C}_{i, n}, \tilde{C}_{j, m}\right]=0=\left[\tilde{C}_{i, m}^{\dagger} \tilde{C}_{j, m}^{\dagger}\right] .}
\end{aligned}
$$

These operations act on the $\alpha$ vacuum state in the following way:

$$
\begin{aligned}
\tilde{c}_{\mathbf{R}}|\alpha\rangle & =\gamma_{p}^{(\alpha)} \tilde{c}_{\mathbf{L}}^{\dagger}|\alpha\rangle, \quad \tilde{c}_{\mathbf{R}}|\alpha\rangle=\gamma_{p}^{(\alpha)} \tilde{c}_{\mathbf{L}}^{\dagger}|\alpha\rangle, \\
\tilde{C}_{\mathbf{R}, n}|\alpha\rangle & =\Gamma_{p, n}^{(\alpha)} \tilde{C}_{\mathbf{L}, n}^{\dagger}|\alpha\rangle, \quad \tilde{C}_{\mathbf{R}, n}|\alpha\rangle=\Gamma_{p, n}^{(\alpha)} \tilde{C}_{\mathbf{L}, n}^{\dagger}|\alpha\rangle .
\end{aligned}
$$

Further, one can express the new $c$ type annihilation operators in terms of the old $b$ type annihilation operators as:

$$
\begin{gathered}
\tilde{c}_{J}=b_{I} \tilde{\mathcal{G}}_{J}^{I}=b_{I}\left(\begin{array}{cc}
\tilde{U}_{q} & \tilde{V}_{q}^{*} \\
\tilde{V}_{q} & \tilde{U}_{q}^{*}
\end{array}\right), \\
\tilde{C}_{J(n)}=\bar{b}_{J(n)}\left(\tilde{\mathcal{G}}_{(n)}\right)_{J}^{I}=\bar{b}_{J(n)}\left(\begin{array}{cc}
\overline{\tilde{U}}_{q, n} & \overline{\tilde{V}}_{\sigma q, n}^{*} \\
\overline{\tilde{V}}_{q, n} & \overline{\tilde{U}}_{q, n}^{*}
\end{array}\right) .
\end{gathered}
$$

Note that $\tilde{U}_{q} \equiv \operatorname{diag}(\tilde{u}, \overline{\tilde{u}}), \tilde{V}_{q} \equiv \operatorname{diag}(\tilde{v}, \overline{\tilde{v}}), \overline{\tilde{U}}_{q, n} \equiv$ $\operatorname{diag}\left(\tilde{U}_{n}, \overline{\tilde{U}}_{n}\right), \overline{\tilde{V}}_{q, n} \equiv \operatorname{diag}\left(\tilde{V}_{n}, \overline{\tilde{V}}_{n}\right)$. From Eqs. (3.106) and (3.111), we obtain the following sets of homogeneous equations: 


$$
\begin{aligned}
& \gamma_{p}^{(\alpha)}=\frac{1}{\sqrt{2}\left|\tilde{m}_{\mathbf{R} \mathbf{L}}\right|}\left[\left(1+\left|\tilde{m}_{\mathbf{R} \mathbf{L}}\right|^{4}+\left|\tilde{m}_{\mathbf{R} \mathbf{R}}\right|^{4}-2\left|\tilde{m}_{\mathbf{R} \mathbf{R}}\right|^{2}-\tilde{m}_{\mathbf{R} \mathbf{R}}^{2}\left(\tilde{m}_{\mathbf{R} \mathbf{L}}^{*}\right)^{2}-\tilde{m}_{\mathbf{R} \mathbf{L}}^{2}\left(\tilde{m}_{\mathbf{R} \mathbf{R}}^{*}\right)^{2}\right)\right. \\
& \left. \pm\left\{\left(-1-\left|\tilde{m}_{\mathbf{R L}}\right|^{4}-\left|\tilde{m}_{\mathbf{R R}}\right|^{4}+2\left|\tilde{m}_{\mathbf{R R}}\right|^{2}+\tilde{m}_{\mathbf{R} \mathbf{R}}^{2}\left(\tilde{m}_{\mathbf{R L}}^{*}\right)^{2}+\tilde{m}_{\mathbf{R L}}^{2}\left(\tilde{m}_{\mathbf{R R}}^{*}\right)^{2}\right)^{2}-4\left|\tilde{m}_{\mathbf{R L}}\right|^{4}\right\}^{\frac{1}{2}}\right]^{\frac{1}{2}} \\
& \approx i \frac{\sqrt{2}\left[\cosh ^{2} \alpha+\sinh ^{2} \alpha e^{2 i \pi \nu}+\sinh 2 \alpha \cos \pi \nu e^{i \pi \nu}\right]}{(\sqrt{\cosh 2 \pi p+\cos 2 \pi \nu} \pm \sqrt{\cosh 2 \pi p+\cos 2 \pi \nu+2})\left(\cosh ^{2} \alpha+\sinh ^{2} \alpha e^{-2 \pi(p-i \nu)}\right)} \\
& \stackrel{\alpha=0}{\longrightarrow} \quad \gamma_{p}^{(0)}=\frac{1}{2 m_{\mathbf{R L}}}\left[\left(1+m_{\mathbf{R} \mathbf{L}}^{2}-m_{\mathbf{R} \mathbf{R}}^{2}\right) \pm \sqrt{\left(1+m_{\mathbf{R} \mathbf{L}}^{2}-m_{\mathbf{R R}}^{2}\right)^{2}-4 m_{\mathbf{R} \mathbf{L}}^{2}}\right] \\
& \approx i \frac{\sqrt{2}}{\sqrt{\cosh 2 \pi p+\cos 2 \pi v} \pm \sqrt{\cosh 2 \pi p+\cos 2 \pi v+2}}, \\
& \Gamma_{p, n}^{(\alpha)}=\frac{1}{\sqrt{2}\left|\tilde{m}_{\mathbf{R} \mathbf{L}, n}\right|}\left[\left(1+\left|\tilde{m}_{\mathbf{R L}, n}\right|^{4}+\left|\tilde{m}_{\mathbf{R R}, n}\right|^{4}\right.\right. \\
& \left.-2\left|\tilde{m}_{\mathbf{R R}, n}\right|^{2}-\tilde{m}_{\mathbf{R} \mathbf{R}, n}^{2}\left(\tilde{m}_{\mathbf{R} \mathbf{L}, n}^{*}\right)^{2}-\tilde{m}_{\mathbf{R} \mathbf{L}, n}^{2}\left(\tilde{m}_{\mathbf{R R}, n}^{*}\right)^{2}\right) \pm\left\{\left(-1-\left|\tilde{m}_{\mathbf{R L}, n}\right|{ }^{\left|m_{\mathbf{R R}, n}\right|^{4}}\right.\right. \\
& \left.\left.+2\left|\tilde{m}_{\mathbf{R R}, n}\right|^{2}+\tilde{m}_{\mathbf{R R}, n}^{2}\left(\tilde{m}_{\mathbf{R} \mathbf{L}, n}^{*}\right)^{2}+\tilde{m}_{\mathbf{R} \mathbf{L}, n}^{2}\left(\tilde{m}_{\mathbf{R}, n}^{*}\right)^{2}\right)^{2}-4\left|\tilde{m}_{\mathbf{R L}, n}\right|^{4}\right\}^{\frac{1}{2}} \\
& \approx i \frac{\sqrt{2}\left[\cosh ^{2} \alpha+\sinh ^{2} \alpha e^{2 i \pi v}+c \operatorname{\eta h} 2 \alpha\right.}{\left(\sqrt{\cosh 2 \pi p_{n}+\cos 2 \pi v} \pm \sqrt{\cosh 2 \pi p_{n}+\cos 2} \pi v\right.} \frac{\left.s \pi v e^{i \pi v}\right]}{\left(\cosh \alpha+\sinh ^{2} \alpha e^{-2 \pi\left(p_{n}-i v\right)}\right)} \\
& \stackrel{\alpha=0}{\longrightarrow} \quad \Gamma_{p, n}^{(0)}=\frac{1}{2 \bar{m}_{\mathbf{R} \mathbf{L}, n}}\left[\left(1+\bar{m}_{\mathbf{R} \mathbf{L}, n}^{2}-\bar{m}_{\mathbf{R}, n}^{2}\right)\right. \\
& \approx i \frac{\sqrt{2}}{\sqrt{\cosh 2 \pi p_{n}+\cos 2 \pi \nu} \pm \sqrt{\cos ^{2} 1}} \frac{1 \pi p_{n}+\cos , ~}{2 \pi v+2},
\end{aligned}
$$

\section{For complementary solution:}

$$
\begin{aligned}
& \tilde{m}_{\mathbf{R} \mathbf{R}} \tilde{u}+\tilde{v}-\gamma_{p}^{(\alpha)} \tilde{m}_{\mathbf{R} \mathbf{L}} \overline{\tilde{v}}^{*}=0, \\
& \tilde{m}_{\mathbf{R} \mathbf{R}} \overline{\tilde{u}}+\overline{\tilde{v}}-\gamma_{p}^{(\alpha)} \tilde{m} \tilde{v}^{*}-0 \\
& \tilde{m}_{\mathbf{R} \mathbf{L}} \tilde{u}-\gamma_{p}^{(\alpha)} \overline{\tilde{u}}^{*}-\gamma_{p}^{(\alpha)}, \overline{\tilde{v}}^{*}=0, \\
& \tilde{m}_{\mathbf{R} \mathbf{L}} \overline{\tilde{u}}-\gamma_{p}^{(\alpha)} \tilde{u}^{*}{ }^{(\alpha)} \tilde{m}_{\mathbf{R} \mathbf{R}} \tilde{v}^{*}=0,
\end{aligned}
$$

\section{For preticular solution:}

$$
\begin{aligned}
& \tilde{U}_{n} \tilde{\mathbf{v}}_{\tilde{V}_{n}-\Gamma_{p, n}^{(\alpha)} \tilde{m}_{\mathbf{R} \mathbf{L}, n} \overline{\tilde{V}}_{n}^{*}=0,}^{(\alpha)} \tilde{m}_{\mathbf{R} \mathbf{L}, n} \tilde{V}_{n}^{*}=0, \\
& \tilde{m}_{\mathbf{R} \mathbf{L}, \eta} \tilde{U}_{n}-\Gamma_{p, n}^{(\alpha)} \overline{\tilde{U}}_{n}^{*}-\Gamma_{p, n}^{(\alpha)} \tilde{m}_{\mathbf{R} \mathbf{R}, n} \overline{\tilde{V}}_{n}^{*}=0, \\
& \tilde{m}_{\mathbf{R} \mathbf{L}, n} \overline{\tilde{U}}_{n}-\Gamma_{p, n}^{(\alpha)} \tilde{U}_{n}^{*}-\Gamma_{p, n}^{(\alpha)} \tilde{m}_{\mathbf{R} \mathbf{R}, n} \tilde{V}_{n}^{*}=0,
\end{aligned}
$$

Using the relations $\tilde{v}^{*}=\overline{\tilde{v}}, \tilde{u}^{*}=\overline{\tilde{u}}, \tilde{V}_{n}^{*}=\overline{\tilde{V}}_{n}, \tilde{U}_{n}^{*}=\overline{\tilde{U}}_{n}$, $|\tilde{u}|^{2}-|\tilde{v}|^{2}=1$ and $\left|\tilde{U}_{n}\right|^{2}-\left|\tilde{V}_{n}\right|^{2}=1$ the solutions of these equations can be written as: where the components $\tilde{m}_{\mathbf{R R}}=\tilde{m}_{\mathbf{L L}}, \tilde{m}_{\mathbf{R} \mathbf{L}}=\tilde{m}_{\mathbf{L R}}$ and $\tilde{m}_{\mathbf{R R}, n}=\tilde{m}_{\mathbf{L L}, n}, \tilde{m}_{\mathbf{R L}, n}=\tilde{m}_{\mathbf{L R}, n}$ are defined in Eqs. $(3.62-$ 68) for general $\alpha$ vacua. Also the components without tilde symbol represent the contribution from $\alpha=0$, which is the Bunch Davies vacuum state.

Further, for the massless $(v=3 / 2)$ axion field we get the following simplified expressions:

$$
\begin{aligned}
& \gamma_{p}^{(\alpha, 3 / 2)} \approx i \frac{\sqrt{2}}{(\sqrt{\cosh 2 \pi p-1} \pm \sqrt{\cosh 2 \pi p+1})\left(\cosh ^{2} \alpha-\sinh ^{2} \alpha e^{-2 \pi p}\right)} \\
& \stackrel{\alpha=0}{\longrightarrow} \quad \gamma_{p}^{(0,3 / 2)} \approx i \frac{\sqrt{2}}{\sqrt{\cosh 2 \pi p-1} \pm \sqrt{\cosh 2 \pi p+1}}, \\
& \Gamma_{p, n}^{(\alpha)} \approx i \frac{\sqrt{2}}{\left(\sqrt{\cosh 2 \pi p_{n}-1} \pm \sqrt{\cosh 2 \pi p_{n}+1}\right)\left(\cosh ^{2} \alpha-\sinh ^{2} \alpha e^{-2 \pi p_{n}}\right)} \\
& \alpha=0 \quad \Gamma_{p, n}^{(0)} \approx i \frac{\sqrt{2}}{\sqrt{\cosh 2 \pi p_{n}-1} \pm \sqrt{\cosh 2 \pi p_{n}+1}},
\end{aligned}
$$

In the large axion mass $\left(v^{2}<0\right.$ where $\left.v \rightarrow-i|v|\right)$ limit the two solutions for the $\gamma_{p}^{(\alpha)}$ and $\Gamma_{p, n}^{(\alpha)}$ for $\alpha$ vacuum are 
given by:

$$
\begin{aligned}
\gamma_{p}^{(\alpha)} \approx & \frac{1}{2\left|\tilde{m}_{\mathbf{R L}}\right|}\left[\left(1+\left|\tilde{m}_{\mathbf{R L}}\right|^{2}-\tilde{m}_{\mathbf{R R}}^{2}\right)\right. \\
& \pm \sqrt{\left.\left(1+\left|\tilde{m}_{\mathbf{R L}}\right|^{2}-\tilde{m}_{\mathbf{R R}}^{2}\right)^{2}-4\left|\tilde{m}_{\mathbf{R L}}\right|^{2}\right]} . \\
\Gamma_{p, n}^{(\alpha)} \approx & \frac{1}{2\left|\tilde{m}_{\mathbf{R L}, n}\right|}\left[\left(1+\left|\tilde{m}_{\mathbf{R L}, n}\right|^{2}-\tilde{m}_{\mathbf{R R}, n}^{2}\right)\right. \\
& \left. \pm \sqrt{\left(1+\left|\tilde{m}_{\mathbf{R L}}\right|^{2}-\tilde{m}_{\mathbf{R R}}^{2}\right)^{2}-4\left|\tilde{m}_{\mathbf{R L}, n}\right|^{2}}\right]
\end{aligned}
$$

In this limit, we divide the total window of $p$ into two regions, given by $0<p<|v|$ and $|v|<p<\Lambda_{\mathbf{C}}$. In these regions of interest, the two solutions for $\gamma_{p}^{(\alpha)}$ in presence of $\alpha$ vacuum can be approximately written as:

$$
\left|\gamma_{p}^{(\alpha)}\right| \approx \begin{cases}e^{\mp \pi|\nu|}(1+\tan \alpha) & \text { for } 0<p<|\nu| \\ \frac{e^{\mp \pi p}(1+\tan \alpha)\left(1+\tan \alpha e^{2 \pi|\nu|}\right)}{\left(1+\tan ^{2} \alpha e^{-2 \pi p}\right)} & \text { for }|\nu|<p<\Lambda_{\mathbf{C}} / 2 \pi .\end{cases}
$$

and

$\left|\Gamma_{p, n}^{(\alpha)}\right|= \begin{cases}e^{\mp \pi|v|}(1+\tan \alpha) & \text { for } 0<p<|\nu| \\ \frac{e^{\mp \pi p_{n}}(1+\tan \alpha)\left(1+\tan \alpha e^{2 \pi|v|}\right)}{\left(1+\tan ^{2} \alpha e^{-2 \pi p_{n}}\right)} & \text { for }|\nu|<p<\end{cases}$

$(3 ., j)$

$$
\begin{aligned}
& \Gamma_{p, n}^{(\alpha, 3 / 2)} \approx i \frac{2 \operatorname{sech}^{2} \alpha}{\left(\sqrt{\left|\cosh 2 \pi p_{n}\right|} \pm \sqrt{\left|\cosh 2 \pi p_{n}\right|+4}\right)} \\
& \stackrel{\alpha=0}{\longrightarrow} \quad \Gamma_{p, n}^{(0,3 / 2)} \approx i \frac{2}{\sqrt{\left|\cosh 2 \pi p_{n}\right|} \pm \sqrt{\left|\cosh 2 \pi p_{n}\right|+4}},
\end{aligned}
$$

On the other hand, in the limit $p<<1$ we results:

$\gamma_{p}^{(\alpha)} \approx i \frac{\sqrt{2}\left[\cosh ^{2} \alpha+\sinh ^{2} \alpha\right.}{(\sqrt{\cos 2 \pi v+1} \pm \sqrt{\cos 2}} \frac{e^{2 i \pi v}+\sinh }{\overline{++3})\left(\cosh 2 \cos ^{2} \alpha+\sinh ^{2} \alpha e^{2 \pi i v}\right)}$

$\stackrel{\alpha=0}{\longrightarrow} \quad \gamma_{p}^{(0)} \approx i \frac{\sqrt{\cos 2 \pi v+}}{2} \frac{\sqrt{2}}{\sqrt{\cos 2 \pi v+3}}$,

$\Gamma_{p, n}^{(\alpha)} \approx i \frac{\sqrt{2}[\cos \Lambda}{(\sqrt{\cos 2 \pi \nu}} \frac{\left.\sinh ^{2} \alpha e^{2 i \pi \nu}+\sinh 2 \alpha \cos \pi v e^{i \pi \nu}\right]}{\sqrt{\mathrm{n} 2 \pi \nu+3})\left(\cosh ^{2} \alpha+\sinh ^{2} \alpha e^{2 \pi i v}\right)}$

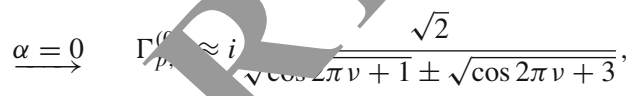

which, io mossless $(v=3 / 2)$ axion field, simplifies to:

Further, in the limit $p>>1$ we get th following samplified

$$
\begin{aligned}
& \gamma_{H}^{, 3 / 2)} \approx \pm i \frac{1}{\sqrt{2}} \stackrel{\alpha=0}{\stackrel{\alpha}{\longrightarrow}} \gamma_{p}^{(0,3 / 2)} \approx \pm i \frac{1}{\sqrt{2}}, \\
& \Gamma_{p, n}^{(\alpha, 3 / 2)} \approx \pm i \frac{1}{\sqrt{2}} \stackrel{\alpha=0}{\stackrel{\Gamma_{p, n}}{\longrightarrow}} \quad \Gamma_{p \pm i \frac{1}{\sqrt{2}}}^{(0,3 / 2)}
\end{aligned}
$$

results:

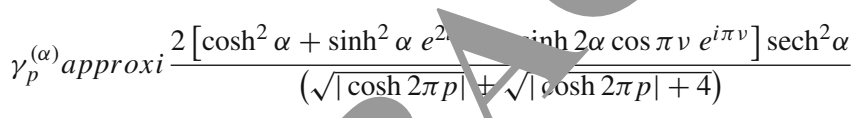

$\stackrel{\alpha=0}{\longrightarrow} \quad \gamma_{p}^{(0)} \approx i \frac{2}{\sqrt{\mid c \sigma s} \mathrm{Cosh} 2 \pi p \mid+4}$,

$\Gamma_{p, n}^{(\alpha)} \approx i \frac{2\left[\cosh ^{2} \alpha\right.}{\left(\sim \sinh ^{2} \alpha\right.} \frac{\left.\pi \nu /+\sinh 2 \alpha \cos \pi v e^{i \pi \nu}\right] \operatorname{sech}^{2} \alpha}{\sin 2 \pi p_{n}} \gamma_{\left. \pm \sqrt{\left|\cosh 2 \pi p_{n}\right|+4}\right)}$

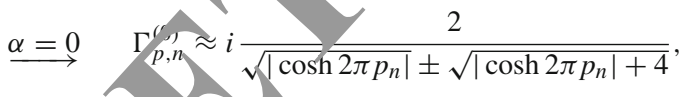

Fo $\mathrm{ma}_{\mathrm{a}}$ sless $(=3 / 2)$ axion field this simplifies to:

$\gamma_{p}^{(\alpha, 3 / 2)} \approx i \frac{2 \operatorname{sech}^{2} \alpha}{(\sqrt{|\cosh 2 \pi p|} \pm \sqrt{|\cosh 2 \pi p|+4})}$

$\stackrel{\alpha=0}{\longrightarrow} \quad \gamma_{p}^{(0,3 / 2)} \approx i \frac{2}{\sqrt{|\cosh 2 \pi p|} \pm \sqrt{|\cosh 2 \pi p|+4}}$, and are very useful information for the computation of spectrum of vacuum fluctuation.

Further, the Fourier mode of the total compact solution in the region $\mathbf{L}$ in case of $\alpha$ vacua can be re-expressed in terms of the oscillators defined in the new basis $(\tilde{c}, \tilde{C})$ as well as the $\mathbf{S O}(\mathbf{1 , 3})$ quantum numbers $(p, l, m)$ as:

$$
\begin{aligned}
& \phi_{\mathbf{L}, p l m}\left(t_{\mathbf{L}}\right)=\frac{H}{\sinh t_{\mathbf{L}}} \tilde{c}_{\mathcal{I}}^{\mathbf{T}} \tilde{\psi}_{\mathbf{T}}^{\mathcal{I}} \\
& =\frac{H}{\sinh t_{\mathbf{L}}}\left[\frac{1}{\mathcal{N}_{p}} \widetilde{\left(G^{-1}\right)_{J}^{I}} \mathcal{P}^{J}+\sum_{n=0}^{\infty} \frac{1}{\mathcal{N}_{p,(n)}} \widetilde{\left(G_{(n)}^{-1}\right)_{J}^{I}} \mathcal{P}_{(n)}^{J}\right],
\end{aligned}
$$

where the total wave function $\tilde{\psi}_{\mathbf{T}}^{\mathcal{I}}$ is a column matrix and for the complementary and particular part of the solution the inverse matrix $\widetilde{\left(G^{-1}\right)_{J}^{I}}$ and $\widetilde{\left(G_{(n)}^{-1}\right)_{J}^{I}}$ are defined as: 


$$
\begin{aligned}
\widetilde{\left(G^{-1}\right)_{J}^{I}} & =\left(\begin{array}{cc}
\tilde{\bar{u}}^{*} & -\tilde{\bar{v}}^{*} \\
-\tilde{\bar{v}} & \tilde{\bar{u}}
\end{array}\right), \\
\widetilde{\left(G_{(n)}^{-1}\right)_{J}^{I}} & =\left(\begin{array}{cc}
\tilde{\bar{U}}_{(n)}^{*} & -\tilde{\bar{V}}_{(n)}^{*} \\
-\tilde{\bar{V}}_{(n)} & \tilde{\bar{U}}_{(n)}
\end{array}\right), \\
\psi^{\mathcal{I}, \mathbf{T}} & =\left(\begin{array}{c}
\psi^{\mathbf{L}, \mathbf{T}}\left(t_{\mathbf{L}}\right) \\
\psi^{\mathbf{L}^{*}, \mathbf{T}}\left(t_{\mathbf{L}}\right)
\end{array}\right) .
\end{aligned}
$$

When we trace out the degrees of freedom over the right part of the Hilbert space, we obtain the following reduced density matrix for the left part of the Hilbert space:

$\left(\rho_{\mathbf{L}}(\alpha)\right)_{p, l, m}=\operatorname{Tr}_{\mathbf{R}}|\alpha\rangle\langle\alpha|$,

where the $\alpha$ vacuum state is written in terms of $\tilde{c}$ type of oscillators as:

$$
\begin{aligned}
|\alpha\rangle \approx & {\left[1-\left(\left|\gamma_{p}^{(\alpha)}\right|^{2}+\sum_{n=0}^{\infty}\left|\Gamma_{p, n}^{(\alpha)}\right|^{2}\right)\right]^{1 / 2} } \\
& \times \exp \left[\gamma_{p}^{(\alpha)} \tilde{c}_{\mathbf{R}}^{\dagger} \tilde{c}_{\mathbf{L}}^{\dagger}+\sum_{n=0}^{\infty} \Gamma_{p, n}^{(\alpha)} \tilde{C}_{\mathbf{R}, n}^{\dagger} \tilde{C}_{\mathbf{L}, n}^{\dagger}\right] \\
& \times\left(\left|\mathbf{R}^{\prime}\right\rangle \otimes\left|\mathbf{L}^{\prime}\right\rangle\right)^{(\alpha)},
\end{aligned}
$$

Substituting Eq. (3.138) in Eq. (3.137), we get the expressio. for the reduced density matrix for the left part of the Hilbert space:

$$
\begin{aligned}
& \left(\rho_{\mathbf{L}}(\alpha)\right)_{p, l, m} \\
& =\underbrace{\frac{\left(1-\left|\gamma_{p}^{(\alpha)}\right|^{2}\right)}{1+f_{p}^{(\alpha)}} \sum_{k=0}^{\infty}\left|\gamma_{p}^{(\alpha)}\right|^{2 k}|k ; \widetilde{p}, m\rangle\langle k ; \tilde{p, l, m}|}_{\text {Complement }}
\end{aligned}
$$

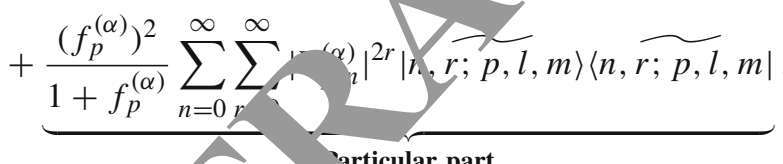

$$
\begin{aligned}
& \text { articular part }
\end{aligned}
$$

where $f^{(\alpha)}$ is oiven by

$f\left(h=\frac{1}{\sum_{n=0}^{\infty}}{ }_{1-\left|\Gamma_{p, n}^{(\alpha)}\right|^{2}}^{1}\right.$,

and the states $|k ; \widetilde{p, l, m}\rangle$ and $|n, \widetilde{r ; p, l}, m\rangle$ are expressed in terms of the new quantum state $\left|\mathbf{L}^{\prime}\right\rangle$ as:

$$
\begin{aligned}
& \left.\left|k \widetilde{p, l, m\rangle}=\frac{1}{\sqrt{k !}}\left(\tilde{c}_{\mathbf{L}}^{\dagger}\right)^{k}\right| \mathbf{L}^{\prime}\right\rangle, \\
& |n, \widetilde{r ; p, l}, m\rangle=\frac{1}{\sqrt{r !}}\left(\tilde{C}_{\mathbf{L}, n}^{\dagger}\right)^{r}\left|\mathbf{L}^{\prime}\right\rangle .
\end{aligned}
$$

Note that for $\alpha=0$, we get back the result obtained for Bunch Davies vacuum.

\subsubsection{Two point correlation function}

In this subsection we explicitly compute the two point correlation function and its significant role to o'stain long range effect in the cosmological correlation usin gen alised $\alpha$ and Bunch Davies vacuum. For this purpose. 1 sing the expression for the reduced density $n$ ix, derlved in the previous subsection, we first comput the $n$ square quantum vacuum fluctuation, which is xpressed fo, $\alpha$ vacua as:

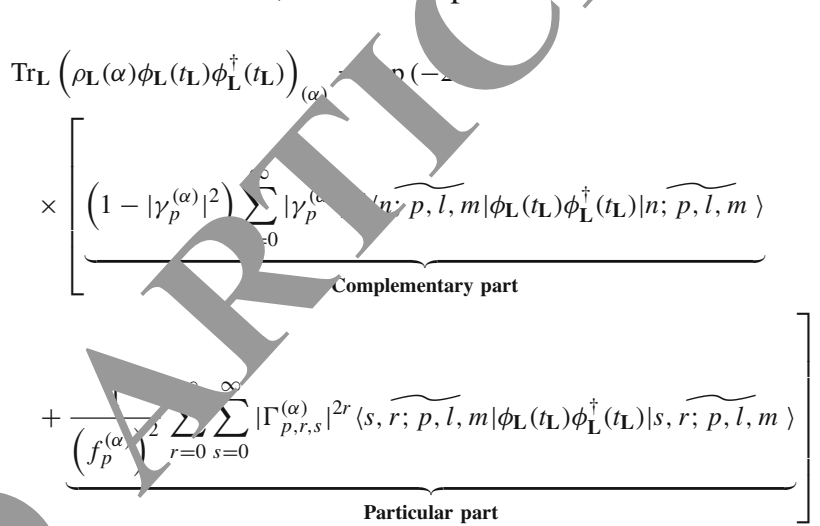

in the above, we have used the shorthand notation $\phi_{\mathbf{L}}\left(t_{\mathbf{L}}\right)=$ $\phi_{\mathbf{L} p l m}(t)$ for the field. Note that, setting $\alpha=0$ in Eq. (3.142) we get the result for the Bunch Davies vacuum which is given by:

$$
\begin{aligned}
\operatorname{Tr}_{\mathbf{L}}\left(\rho_{\mathbf{L}}(\alpha) \phi_{\mathbf{L}}\left(t_{\mathbf{L}}\right) \phi_{\mathbf{L}}^{\dagger}\left(t_{\mathbf{L}}\right)\right)_{(\mathbf{B D})} \\
=\underbrace{\left(1-\left|\gamma_{p}^{(0)}\right|^{2}\right) \sum_{n=0}^{\infty}\left|\gamma_{p}^{(0)}\right|^{2 n}\left\langle n ; p, l, m\left|\phi_{\mathbf{L}}\left(t_{\mathbf{L}}\right) \phi_{\mathbf{L}}^{\dagger}\left(t_{\mathbf{L}}\right)\right| n ; p, l, m\right\rangle}_{\text {Complementary part }} \\
+\underbrace{\frac{1}{\left(f_{p}^{(0)}\right)^{2}} \sum_{r=0}^{\infty} \sum_{s=0}^{\infty}\left|\Gamma_{p, r, s}^{(0)}\right|^{2 r}\left\langle s, r ; p, l, m\left|\phi_{\mathbf{L}}\left(t_{\mathbf{L}}\right) \phi_{\mathbf{L}}^{\dagger}\left(t_{\mathbf{L}}\right)\right| s, r ; p, l, m\right\rangle}_{\text {Particular part }} .
\end{aligned}
$$

Here $|s, r ; p, l, m\rangle$ is the Bunch Davies counterpart of the quantum state in the newly Bogoliubov transformed basis and is obtained by simply setting $\alpha=0$ in the definition of the quantum state introduced in terms of the new oscillators.

The contributions from the complementary and the particular part, as appearing in the right hand side of Eq. (3.142) for each $n$-particle state are found to be:

$$
\begin{aligned}
& \left\langle n ; \widetilde{p, l, m}\left|\phi_{\mathbf{L}}\left(t_{\mathbf{L}}\right) \phi_{\mathbf{L}}^{\dagger}\left(t_{\mathbf{L}}\right)\right| n ; \widetilde{p, l, m}\right\rangle \\
& \quad=\frac{H^{2}}{\sinh ^{2} t_{\mathbf{L}}} \frac{1}{n !}\left\langle\mathbf{L}^{\prime}\left|\left(\tilde{c}_{\mathbf{L}}\right)^{n}\left(\tilde{c}_{\mathcal{I}}^{\mathbf{T}} \tilde{\psi}_{\mathbf{T}}^{\dagger \mathcal{I}}\right)\left(\tilde{c}_{\mathcal{J}}^{\mathbf{T}} \tilde{\psi}_{\mathbf{T}}^{\dagger \mathcal{J}}\right)^{\dagger}\left(\tilde{c}_{\mathbf{L}}^{\dagger}\right)^{n}\right| \mathbf{L}^{\prime}\right\rangle
\end{aligned}
$$




$$
\begin{aligned}
& =\frac{H^{2}}{\sinh ^{2} t_{\mathbf{L}}}(2 n+1)\left|\tilde{\psi}_{\mathbf{T}}^{\mathbf{L}}\right|^{2}, \\
\left\langle s, r ; p, l, m\left|\phi_{\mathbf{L}}\left(t_{\mathbf{L}}\right) \phi_{\mathbf{L}}^{\dagger}\left(t_{\mathbf{L}}\right)\right| s, \widetilde{r ; p, l, m\rangle}\right. & =\frac{H^{2}}{\sinh ^{2} t_{\mathbf{L}}} \frac{1}{r !}\left\langle\mathbf{L}^{\prime}\left|\left(\tilde{C}_{\mathbf{L}}^{(s)}\right)^{r}\left(\tilde{c}_{\mathcal{I}}^{\mathbf{T}} \tilde{\psi}_{\mathbf{T}}^{\dagger \mathcal{I}}\right)\left(\tilde{c}_{\mathcal{J}}^{\mathbf{T}} \tilde{\psi}_{\mathbf{T}}^{\dagger \mathcal{J}}\right)^{\dagger}\left(\tilde{C}_{\mathbf{L}}^{(s) \dagger}\right)^{r}\right| \mathbf{L}^{\prime}\right\rangle \\
= & \frac{H^{2}}{\sinh ^{2} t_{\mathbf{L}}}(2 r+1)\left|\tilde{\psi}_{\mathbf{T}}^{\mathbf{L}}\right|^{2},
\end{aligned}
$$

where $\tilde{\psi}_{\mathbf{T}}^{\mathbf{L}}$ is given by:

$$
\begin{aligned}
\tilde{\psi}_{\mathbf{T}}^{\mathbf{L}}= & \left(\begin{array}{c}
\tilde{\psi}_{\mathbf{T}}^{\mathbf{L}}(t) \\
\tilde{\psi}_{\mathbf{T}}^{\mathbf{L}}(t)
\end{array}\right)=\left(\begin{array}{c}
\mathcal{E}_{\mathbf{L}} \widetilde{\mathcal{P}}^{\mathbf{L}}+\mathcal{F}_{\mathbf{L}} \widetilde{\mathcal{P}}^{\mathbf{L} *} \\
\mathcal{F}_{\mathbf{L}}^{*} \widetilde{\mathcal{P}}^{\mathbf{L}}+\mathcal{E}_{\mathbf{L}}^{*} \widetilde{\mathcal{P}}^{\mathbf{L} *}
\end{array}\right) \\
& +\sum_{n=0}^{\infty}\left(\begin{array}{c}
\mathcal{E}_{\mathbf{L},(n)} \\
\widetilde{\mathcal{P}}_{(n)}^{\mathbf{L}}+\mathcal{F}_{\mathbf{L},(n)} \underset{\widetilde{\mathcal{P}}_{(n)}^{\mathbf{L} *}}{\mathcal{F}_{\mathbf{L},(n)}^{*}} \widetilde{\mathcal{P}}_{(n)}^{\mathbf{L}}+\mathcal{E}_{\mathbf{L},(n)}^{*} \widetilde{\mathcal{P}}_{(n)}^{\mathbf{L} *}
\end{array}\right),
\end{aligned}
$$

with the entries of the column matrix for the complementary and particular integral part of the solution being:

$$
\begin{aligned}
\mathcal{E}_{\mathbf{L}} & =\frac{\overline{\tilde{u}}}{\mathcal{N}_{c}}, \\
\mathcal{F}_{\mathbf{L}} & =-\frac{\overline{\tilde{v}}}{\mathcal{N}_{c}}, \\
\mathcal{E}_{\mathbf{L},(n)} & =\frac{\overline{\tilde{U}}_{n}}{\mathcal{N}_{c,(n)}}, \\
\mathcal{F}_{\mathbf{L},(n)} & =-\frac{\overline{\tilde{V}}}{\mathcal{N}_{c,(n)}} .
\end{aligned}
$$

The normalization constants $\mathcal{N}_{c}$ and $\mathcal{N}_{\text {, }}$ for th ormplementary part and particular integral art of the solution is defined as:



$$
\begin{aligned}
& \mathcal{N}_{c,(n)}=\sqrt{\frac{2}{\pi}} e^{-\frac{\pi p_{n}}{2}}, \cos 2 \pi p_{n}+\cos 2 \pi v .
\end{aligned}
$$

The expressio for $(\overline{\tilde{u}}, \hat{v}$ ) or complementary solution and $\left(\overline{\tilde{U}}_{n}, \overline{\tilde{V}}_{n}\right)$ for particu solution are given by the following expressiors:

For comp. ents part:

$$
\begin{aligned}
& \hat{\tilde{u}}=\frac{1-\gamma_{p}^{(\alpha)} \tilde{m}_{\mathbf{L R}}}{\sqrt{\lambda_{1}-\left.\gamma_{p}^{(\alpha)} \tilde{m}_{\mathbf{L R}}\right|^{2}-\left|\tilde{m}_{\mathbf{R} \mathbf{R}}\right|^{2}}} \stackrel{\alpha=0}{\longrightarrow} \\
& \bar{u}=\frac{1-\gamma_{p}^{(0)} m_{\mathbf{L R}}}{\sqrt{\left|1-\gamma_{p}^{(0)} m_{\mathbf{L R}}\right|^{2}-\left|m_{\mathbf{R R}}\right|^{2}}}, \\
& \overline{\tilde{v}}=\frac{\tilde{m}_{\mathbf{R R}}}{\sqrt{\left|1-\gamma_{p}^{(\alpha)} \tilde{m}_{\mathbf{L R}}\right|^{2}-\left|\tilde{m}_{\mathbf{R R}}\right|^{2}}} \stackrel{\alpha=0}{\longrightarrow}
\end{aligned}
$$

$$
\overline{\tilde{v}}=\frac{m_{\mathbf{R R}}}{\sqrt{\left|1-\gamma_{p}^{(0)} m_{\mathbf{L R}}\right|^{2}-\left|m_{\mathbf{R R}}\right|^{2}}}
$$

\section{For particular part:}

$$
\begin{aligned}
& \overline{\tilde{U}}_{n}=\frac{1-\Gamma_{p, n}^{(\alpha)} \tilde{m}_{\mathbf{L R}}}{\sqrt{\left|1-\Gamma_{p, n}^{(\alpha)} \tilde{m}_{\mathbf{L R}}\right|^{2}-\left|\tilde{m}_{\mathbf{R} \mathbf{R}}\right|^{2}}} \quad \alpha=0 \\
& \bar{U}_{n}=\frac{1-\Gamma_{p, n}^{(0)} m_{\mathbf{L R}}}{\sqrt{\left|1-\Gamma_{p, n}^{(0)} m_{\mathbf{L R}}\right|^{2}-\mid m_{\mathbf{R}} \mathbf{p}^{\prime 2}}} \\
& \left.\overline{\tilde{V}}_{n}=\frac{\tilde{m}_{\mathbf{L R}}}{\sqrt{\left|1-\Gamma_{p, n}^{(\alpha)} \tilde{m}_{\mathbf{L R}}\right|^{2} \mid \hat{m}_{\mathbf{L}}}} \quad \alpha\right)=0
\end{aligned}
$$

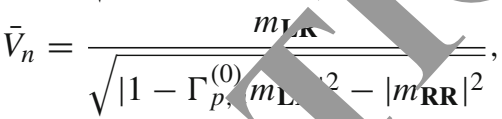

$$
\text { Resu1 } \text { gener.dised } \alpha \text { vacua }
$$$$
\text { Results Bunch Davies vacuum. }
$$

where the expression for $\left(\tilde{m}_{\mathbf{L R}}, \tilde{m}_{\mathbf{R} \mathbf{R}}\right)$ and $\left(\gamma_{p}^{(\alpha)}, \Gamma_{p, n}^{(\alpha)}\right)$ for the complementary and particular part of the solution are 'efined earlier in Eqs. (3.62-68) and Eqs. (3.119-3.120) r. bectively. We have used Eqs. (3.113), (3.114), (3.115) and (3.116) and also have imposed the normalization conditions, $|\tilde{u}|^{2}-\left.\overline{\tilde{v}}\right|^{2}=1$ and $|\overline{\tilde{u}}|^{2}-\left.\overline{\tilde{v}}\right|^{2}=1$. Note that the structural form of the equations for $\alpha=0$ corresponding to Bunch Davies vacuum is exactly same as that of $\alpha$ vacua. Only the significant changes appear when we explicitly consider the entries of $\left(m_{\mathbf{L R}}, m_{\mathbf{R R}}\right)$ and $\left(\gamma_{p}, \Gamma_{p, n}\right)$ for the complementary and particular part of the solution.

Now, substituting Eqs. (3.144) and (3.145) in Eq. (3.142) we get the following simplified expression for the mean square quantum vacuum fluctuation for $\alpha$ vacua as:

$$
\begin{aligned}
& \operatorname{Tr}_{\mathbf{L}}\left(\rho_{\mathbf{L}}(\alpha) \phi_{\mathbf{L}}\left(t_{\mathbf{L}}\right) \phi_{\mathbf{L}}^{\dagger}\left(t_{\mathbf{L}}\right)\right)_{(\alpha)} \\
& =\exp (-2 \alpha)[\underbrace{\frac{H^{2}}{\sinh ^{2} t_{\mathbf{L}}}\left|\tilde{\psi}_{\mathbf{T}}^{\mathbf{L}}\right|^{2}\left(1-\left|\gamma_{p}^{(\alpha)}\right|^{2}\right) \sum_{n=0}^{\infty}\left|\gamma_{p}^{(\alpha)}\right|^{2 n}(2 n+1)}_{\text {Complementary part }} \\
& +\underbrace{\frac{H^{2}}{\sinh ^{2} t_{\mathbf{L}}}\left|\tilde{\psi}_{\mathbf{T}}^{\mathbf{L}}\right|^{2} \frac{1}{\left(f_{p}^{(\alpha)}\right)^{2}} \sum_{r=0}^{\infty} \sum_{s=0}^{\infty}\left|\Gamma_{p, r, s}^{(\alpha)}\right|^{2 r}(2 r+1)}_{\text {Particular part }}] . \\
& =\frac{H^{2}}{\sinh ^{2} t_{\mathbf{L}}}\left|\tilde{\psi}_{\mathbf{T}}^{\mathbf{L}}\right|^{2} \exp (-2 \alpha) \\
& \times\left[\frac{1+\left|\gamma_{p}^{(\alpha)}\right|^{2}}{1-\left|\gamma_{p}^{(\alpha)}\right|^{2}}+\frac{1}{\left(f_{p}^{(\alpha)}\right)^{2}} \sum_{s=0}^{\infty} \frac{1+\left|\Gamma_{p, s}^{(\alpha)}\right|^{2}}{\left(1-\left|\Gamma_{p, s}^{(\alpha)}\right|^{2}\right)^{2}}\right] .
\end{aligned}
$$


Setting $\alpha=0$ we get the expression for the Bunch Davies vacuum as:

$$
\begin{aligned}
\operatorname{Tr}_{\mathbf{L}} & \left(\rho_{\mathbf{L}}(\alpha) \phi_{\mathbf{L}}\left(t_{\mathbf{L}}\right) \phi_{\mathbf{L}}^{\dagger}\left(t_{\mathbf{L}}\right)\right)_{(\mathbf{B D})} \\
= & \underbrace{\frac{H^{2}}{\sinh ^{2} t_{\mathbf{L}}}\left|\psi_{\mathbf{T}}^{\mathbf{L}}\right|^{2}\left(1-\left|\gamma_{p}^{(0)}\right|^{2}\right) \sum_{n=0}^{\infty}\left|\gamma_{p}^{(0)}\right|^{2 n}(2 n+1)}_{\text {Complementary part }} \\
& +\underbrace{\frac{H^{2}}{\sinh ^{2} t_{\mathbf{L}}}\left|\psi_{\mathbf{T}}^{\mathbf{L}}\right|^{2} \frac{1}{\left(f_{p}^{(0)}\right)^{2}} \sum_{r=0}^{\infty} \sum_{s=0}^{\infty}\left|\Gamma_{p, r, s}^{(0)}\right|^{2 r}(2 r+1) .}_{\text {Particular part }} \\
= & \frac{H^{2}}{\sinh ^{2} t_{\mathbf{L}}}\left|\psi_{\mathbf{T}}^{\mathbf{L}}\right|^{2}\left[\frac{1+\left|\gamma_{p}^{(0)}\right|^{2}}{1-\left|\gamma_{p}^{(0)}\right|^{2}}+\frac{1}{\left(f_{p}^{(0)}\right)^{2}} \sum_{s=0}^{\infty} \frac{1+\left|\Gamma_{p, s}^{(0)}\right|^{2}}{\left(1-\left|\Gamma_{p, s}^{(0)}\right|^{2}\right)^{2}}\right] .
\end{aligned}
$$

We note that, to derive this expression we have used the following identities:

$$
\begin{aligned}
& \sum_{n=0}^{\infty}(2 n+1)\left|\gamma_{p}^{(\alpha)}\right|^{2 n} \\
& =\frac{1+\left|\gamma_{p}^{(\alpha)}\right|^{2}}{\left(1-\left.\gamma_{p}^{(\alpha)}\right|^{2}\right)^{2}} \stackrel{\alpha=0}{\longrightarrow} \sum_{n=0}^{\infty}(2 n+1)\left|\gamma_{p}^{(0)}\right|^{2 n} \\
& =\frac{1+\left|\gamma_{p}^{(0)}\right|^{2}}{\left(1-\left.\gamma_{p}^{(0)}\right|^{2}\right)^{2}} \\
& \sum_{s=0}^{\infty} \sum_{r=0}^{\infty}(2 r+1)\left|\Gamma_{p, r, s}^{(\alpha)}\right|^{2 r} \\
& =\sum_{s=0}^{\infty} \frac{1+\left|\Gamma_{p, s}^{(\alpha)}\right|^{2}}{\left(1-\left.\Gamma_{p, s}^{(\alpha)}\right|^{2}\right)^{2}} \stackrel{\alpha=0}{\longrightarrow} \sum_{s=0}^{\infty} \underset{r=0}{\infty}(2 r+\mid 1)\left|\Gamma_{p, r, s}^{(0)}\right|^{2 r} \\
& =\sum_{s=0}^{\infty} \frac{1+\left|\Gamma_{p, s}^{(0)}\right|^{2}}{\left(1-\left.\Gamma_{p, s}^{(0)}\right|^{2}\right)^{2}} \\
& \left|\tilde{\psi}_{\mathbf{T}}^{\mathbf{L}}\right|^{2}=\left.{\tilde{\tau_{s}} \mathbf{T}}^{\mathbf{L}}\right|^{\dagger} \tilde{\psi}_{\mathbf{T}}^{\mathbf{L}} \\
& -\left[\left.{ }_{\mathbf{L}}\right|^{2}+\left.\mathcal{F}_{\mathbf{L}}\right|^{2}\right) \widetilde{\mathcal{P}}^{\mathbf{L}} \widetilde{\mathcal{P}}^{\mathbf{L} *}+\mathcal{E}_{\mathbf{L}} \mathcal{F}_{\mathbf{L}}^{*}\left(\widetilde{\mathcal{P}}^{\mathbf{L}}\right)^{2}+\mathcal{E}_{\mathbf{L}}^{*} \mathcal{F}_{\mathbf{L}}\left(\widetilde{\mathcal{P}}^{\mathbf{L} *}\right)^{2} \\
& +\underset{n=0}{\infty}\left\{\left(\mathcal{E}_{\mathbf{L}} \mathcal{E}_{\mathbf{L},(n)}^{*}+\mathcal{F}_{\mathbf{L}} \mathcal{F}_{\mathbf{L},(n)}^{*}\right) \widetilde{\mathcal{P}}^{\mathbf{L}} \widetilde{\mathcal{P}}_{(n)}^{\mathbf{L} *}\right. \\
& +\left(\mathcal{E}_{\mathbf{L}} \mathcal{F}_{\mathbf{L},(n)}^{*}+\mathcal{E}_{\mathbf{L},(n)} \mathcal{F}_{\mathbf{L}}^{*}\right) \widetilde{\mathcal{P}}^{\mathbf{L}} \widetilde{\mathcal{P}}_{(n)}^{\mathbf{L}} \\
& \left.+\left(\mathcal{E}_{\mathbf{L},(n)}^{*} \mathcal{F}_{\mathbf{L}}+\mathcal{E}_{\mathbf{L}}^{*} \mathcal{F}_{\mathbf{L},(n)}\right) \widetilde{\mathcal{P}}_{(n)}^{\mathbf{L} *} \widetilde{\mathcal{P}}^{\mathbf{L} *}\right\} \\
& +\sum_{n=0}^{\infty} \sum_{m=0}^{\infty}\left\{\left(\mathcal{E}_{\mathbf{L},(n)} \mathcal{E}_{\mathbf{L},(m)}^{*}+\mathcal{F}_{\mathbf{L},(n)} \mathcal{F}_{\mathbf{L},(m)}^{*}\right) \widetilde{\mathcal{P}}_{(n)}^{\mathbf{L}} \widetilde{\mathcal{P}}_{(m)}^{\mathbf{L} *}\right.
\end{aligned}
$$

$$
\left.\left.+\mathcal{E}_{\mathbf{L},(n)} \mathcal{F}_{\mathbf{L},(m)}^{*} \widetilde{\mathcal{P}}_{(n)}^{\mathbf{L}} \widetilde{\mathcal{P}}_{(m)}^{\mathbf{L}}+\mathcal{E}_{\mathbf{L},(n)}^{*} \mathcal{F}_{\mathbf{L},(m)} \widetilde{\mathcal{P}}_{(n)}^{\mathbf{L} *} \widetilde{\mathcal{P}}_{(m)}^{\mathbf{L} *}\right\}\right]
$$

Here also by fixing the parameter $\alpha=0$ one can get the expression for the square of the magnitude of the wave function for Bunch Davies vacuum in the newly $r$ fined Bogliubov transformed basis.

Using Eq. (3.161), the amplitude of the no liser power spectrum of axion from the generalised $\alpha$ va a can be expressed in all time scales of region as:

$$
\begin{aligned}
& \mathcal{P}\left(p, \alpha, t_{\mathbf{L}}\right)=\frac{p^{3}}{2 \pi^{2}} \operatorname{Tr}_{\mathbf{L}}\left(\rho_{\mathbf{L}}(\alpha)\left(t_{\mathbf{L}}\right) \phi_{\mathbf{L}}^{\dagger}\left(t_{L}\right)\right)_{(\alpha)} \\
& =\frac{p^{3}}{2 \pi^{2}} \frac{H^{2}}{\sinh ^{2} t_{\mathbf{L}}} \mid \tilde{\psi}_{\mathbf{T}}^{\mathbf{L}}{ }^{2} \exp \left({ }^{2}\right) \\
& \times\left[\frac{1+\left|\gamma_{p}^{(\alpha)}\right|^{\alpha}}{\left.1-\gamma_{p}^{(\alpha)}\right)}+\frac{Y}{\left(f_{p}^{(\alpha)}\right)^{2}} \sum_{s=0}^{\infty} \frac{1+\left|\Gamma_{p, s}^{(\alpha)}\right|^{2}}{\left(1-\left|\Gamma_{p, s}^{(\alpha)}\right|^{2}\right)^{2}}\right] \\
& =\frac{p^{3}}{2} \frac{H}{\operatorname{inh}^{2} t_{\mathrm{L}}} \times \mathrm{p}(-2 \alpha) \\
& \times\left[\frac{+\left|\gamma_{p}^{(\alpha)}\right|^{2}}{1-\left|\gamma_{p}^{(\alpha)}\right|^{2}}+\frac{1}{\left(f_{p}^{(\alpha)}\right)^{2}} \sum_{s=0}^{\infty} \frac{1+\left|\Gamma_{p, s}^{(\alpha)}\right|^{2}}{\left(1-\left|\Gamma_{p, s}^{(\alpha)}\right|^{2}\right)^{2}}\right] \\
& \times\left[\left(\left|\mathcal{E}_{\mathbf{L}}\right|^{2}+\left|\mathcal{F}_{\mathbf{L}}\right|^{2}\right) \widetilde{\mathcal{P}}^{\mathbf{L}} \widetilde{\mathcal{P}}^{\mathbf{L} *}+\mathcal{E}_{\mathbf{L}} \mathcal{F}_{\mathbf{L}}^{*}\left(\widetilde{\mathcal{P}}^{\mathbf{L}}\right)^{2}+\mathcal{E}_{\mathbf{L}}^{*} \mathcal{F}_{\mathbf{L}}\left(\widetilde{\mathcal{P}}^{\mathbf{L} *}\right)^{2}\right. \\
& +\sum_{n=0}^{\infty}\left\{\left(\mathcal{E}_{\mathbf{L}} \mathcal{E}_{\mathbf{L},(n)}^{*}+\mathcal{F}_{\mathbf{L}} \mathcal{F}_{\mathbf{L},(n)}^{*}\right) \widetilde{\mathcal{P}}^{\mathbf{L}} \widetilde{\mathcal{P}}_{(n)}^{\mathbf{L} *}\right. \\
& +\left(\mathcal{E}_{\mathbf{L}} \mathcal{F}_{\mathbf{L},(n)}^{*}+\mathcal{E}_{\mathbf{L},(n)} \mathcal{F}_{\mathbf{L}}^{*}\right) \widetilde{\mathcal{P}}^{\mathbf{L}} \widetilde{\mathcal{P}}_{(n)}^{\mathbf{L}}
\end{aligned}
$$

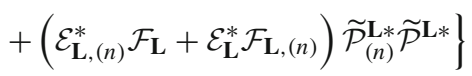

$$
\begin{aligned}
& +\sum_{n=0}^{\infty} \sum_{m=0}^{\infty}\left\{\left(\mathcal{E}_{\mathbf{L},(n)} \mathcal{E}_{\mathbf{L},(m)}^{*}+\mathcal{F}_{\mathbf{L},(n)} \mathcal{F}_{\mathbf{L},(m)}^{*}\right) \widetilde{\mathcal{P}}_{(n)}^{\mathbf{L}} \widetilde{\mathcal{P}}_{(m)}^{\mathbf{L} *}\right. \\
& \left.\left.+\mathcal{E}_{\mathbf{L},(n)} \mathcal{F}_{\mathbf{L},(m)}^{*} \widetilde{\mathcal{P}}_{(n)}^{\mathbf{L}} \widetilde{\mathcal{P}}_{(m)}^{\mathbf{L}}+\mathcal{E}_{\mathbf{L},(n)}^{*} \mathcal{F}_{\mathbf{L},(m)} \widetilde{\mathcal{P}}_{(n)}^{\mathbf{L} *} \widetilde{\mathcal{P}}_{(m)}^{\mathbf{L} *}\right\}\right] .
\end{aligned}
$$

However, the above equation is very complicated to extract any physical information for further cosmological predictions. For this reason we consider the superhorizon time scales $\left(t_{\mathbf{L}}>>1\right)$ of region $\mathbf{L}$, in which the Legendre functions appearing in the complementary part and the particular integral part of the time dependent solution can be approximated as the following simplified form:

$$
\begin{aligned}
& \left(\widetilde{\mathcal{P}}^{\mathbf{L}}, \widetilde{\mathcal{P}}^{\mathbf{L} *}\right) \equiv P_{\nu-\frac{1}{2}}^{ \pm i p}\left(\cosh t_{\mathbf{L}}\right) \\
& \stackrel{t_{\mathbf{L}}>>1}{\longrightarrow} \frac{2^{v-\frac{1}{2}}\left(\cosh t_{\mathbf{L}}\right)^{v-\frac{1}{2}} \Gamma(v)}{\sqrt{\pi} \Gamma\left(v \mp i p+\frac{1}{2}\right)},
\end{aligned}
$$




$$
\begin{aligned}
&\left(\widetilde{\mathcal{P}}_{(n)}^{\mathbf{L}}, \widetilde{\mathcal{P}}_{(n)}^{\mathbf{L} *}\right) \equiv P_{\nu-\frac{1}{2}}^{ \pm i p_{n}}\left(\cosh t_{\mathbf{L}}\right) \\
& \stackrel{t_{\mathbf{L}}>>1}{\longrightarrow} \frac{2^{\nu-\frac{1}{2}}\left(\cosh t_{\mathbf{L}}\right)^{\nu-\frac{1}{2}} \Gamma(v)}{\sqrt{\pi} \Gamma\left(v \mp i p_{n}+\frac{1}{2}\right)} .
\end{aligned}
$$

Consequently, in the superhorizon time scales $\left(t_{\mathbf{L}}>>1\right)$ of region $\mathbf{L}$ Eq. (3.162) can be simplified for as:

$$
\left|\tilde{\psi}_{\mathbf{T}}^{\mathbf{L}}\right|^{2}=\left(\tilde{\psi}_{\mathbf{T}}^{\mathbf{L}}\right)^{\dagger} \tilde{\psi}_{\mathbf{T}}^{\mathbf{L}} \stackrel{t_{\mathbf{L}}>>1}{\mathcal{Q}(p, \alpha, v)}\left(\cosh t_{\mathbf{L}}\right)^{2 v-1}
$$

where the time independent function $\widetilde{\mathcal{Q}(p, \alpha, v)}$ for generalised $\alpha$ vacua is defined as:

$$
\begin{aligned}
& \widetilde{\mathcal{Q}(p, \alpha, v)}=\frac{2^{2 v-1}(\Gamma(v))^{2}}{\pi}\left[\frac{\left(\left|\mathcal{E}_{\mathbf{L}}\right|^{2}+\left|\mathcal{F}_{\mathbf{L}}\right|^{2}\right)}{\left|\Gamma\left(v+i p+\frac{1}{2}\right)\right|^{2}}\right. \\
& +\frac{\mathcal{E}_{\mathbf{L}} \mathcal{F}_{\mathbf{L}}^{*}}{\left(\Gamma\left(v-i p+\frac{1}{2}\right)\right)^{2}}+\frac{\mathcal{E}_{\mathbf{L}}^{*} \mathcal{F}_{\mathbf{L}}}{\left(\Gamma\left(v+i p+\frac{1}{2}\right)\right)^{2}} \\
& +\sum_{n=0}^{\infty}\left\{\frac{\left(\mathcal{E}_{\mathbf{L}} \mathcal{E}_{\mathbf{L},(n)}^{*}+\mathcal{F}_{\mathbf{L}} \mathcal{F}_{\mathbf{L},(n)}^{*}\right)}{\Gamma\left(v-i p+\frac{1}{2}\right) \Gamma\left(v+i p_{n}+\frac{1}{2}\right)}\right. \\
& +\frac{\left(\mathcal{E}_{\mathbf{L}} \mathcal{F}_{\mathbf{L},(n)}^{*}+\mathcal{E}_{\mathbf{L},(n)} \mathcal{F}_{\mathbf{L}}^{*}\right)}{\Gamma\left(v-i p+\frac{1}{2}\right) \Gamma\left(v-i p_{n}+\frac{1}{2}\right)} \\
& +\frac{\left(\mathcal{E}_{\mathbf{L},(n)}^{*} \mathcal{F}_{\mathbf{L}}+\mathcal{E}_{\mathbf{L}}^{*} \mathcal{F}_{\mathbf{L},(n)}\right)}{\Gamma\left(v+i p+\frac{1}{2}\right) \Gamma\left(v+i p_{n}\right.} \\
& +\sum_{n=0}^{\infty} \sum_{m=0}^{\infty}\left\{\frac{\left(\mathcal{E}_{\mathbf{L},(n)} \mathcal{E}_{\mathbf{L}}^{*}(m)+\mathcal{F}_{\mathbf{L},(n)}{ }_{\mathbf{L},(m)}^{*}\right)}{\Gamma\left(v-i p_{n}\right.} \frac{1}{2) \Gamma\left(j+i p_{m}+\frac{1}{2}\right)}\right.
\end{aligned}
$$

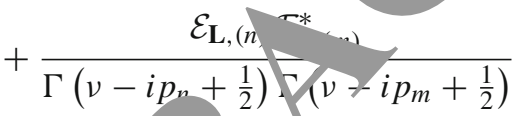

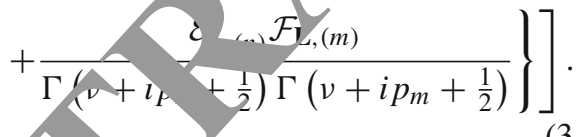

As a resy 4 , in the superhorizon time scales $\left(t_{\mathbf{L}}>>1\right)$ of region $\mathbf{L}$ impli ade of the normalised power spectrum of axi om g. alised $\alpha$ vacua can be expressed as:

$$
\begin{aligned}
& \mathcal{P}(p, \imath, \mathbf{L})=\frac{p^{3}}{2 \pi^{2}} \frac{H^{2}}{\sinh ^{2} t_{\mathbf{L}}}\left|\tilde{\psi}_{\mathbf{T}}^{\mathbf{L}}\right|^{2} \exp (-2 \alpha) \\
& \times\left[\frac{1+\left|\gamma_{p}^{(\alpha)}\right|^{2}}{1-\left|\gamma_{p}^{(\alpha)}\right|^{2}}+\frac{1}{\left(f_{p}^{(\alpha)}\right)^{2}} \sum_{s=0}^{\infty} \frac{1+\left|\Gamma_{p, s}^{(\alpha)}\right|^{2}}{\left(1-\left|\Gamma_{p, s}^{(\alpha)}\right|^{2}\right)^{2}}\right] \\
& \stackrel{t_{\mathbf{L}}>>1}{\longrightarrow} 2 \frac{p^{3}}{2 \pi^{2}} \frac{\left(\cosh t_{\mathbf{L}}\right)^{2 v-1}}{\sinh ^{2} t_{\mathbf{L}}} H^{2} \widetilde{\mathcal{Q}(p, v)} \exp (-2 \alpha)
\end{aligned}
$$

$$
\times\left[\frac{1+\left|\gamma_{p}^{(\alpha)}\right|^{2}}{1-\left|\gamma_{p}^{(\alpha)}\right|^{2}}+\frac{1}{\left(f_{p}^{(\alpha)}\right)^{2}} \sum_{s=0}^{\infty} \frac{1+\left|\Gamma_{p, s}^{(\alpha)}\right|^{2}}{\left(1-\left|\Gamma_{p, s}^{(\alpha)}\right|^{2}\right)^{2}}\right]
$$

We note that in the superhorizon time scales $>>1$ ) of region $\mathbf{L}$ if we consider the massless case $b$ fix ing the mass parameter $v=3 / 2$, then the time dependent $\Delta$ ribu ion can be approximated as:

$$
\left(\frac{\left(\cosh t_{\mathbf{L}}\right)^{2 \nu-1}}{\sinh ^{2} t_{\mathbf{L}}}\right)_{\nu=3 / 2} \stackrel{t_{\mathbf{L}}>1}{\rightarrow} 1
$$

From this we infer that or arbitrary value of the parameter $v$ we can write:

$$
\left(\frac{\left(\cosh t_{\mathbf{L}}\right)^{2 v-1}}{\sinh ^{2}} \stackrel{t_{\mathbf{L}}>1}{\longrightarrow}\left(\cosh t_{\mathbf{L}}\right)^{2 v-3} .\right.
$$

Consequently, h che super horizon time scales $\left(t_{\mathbf{L}}>>1\right)$ of regi ${ }_{1}$ asidering the massless case $(v=3 / 2)$ the amplituc of the normalised power spectrum of axion from generalis $\alpha \alpha$ vacua can be expressed as:

$$
\begin{gathered}
\mathcal{P}\left(p, \alpha, t_{\mathbf{L}}\right)=\frac{p^{3}}{2 \pi^{2}} \frac{H^{2}}{\sinh ^{2} t_{\mathbf{L}}}\left|\tilde{\psi}_{\mathbf{T}}^{\mathbf{L}}\right|^{2} \exp (-2 \alpha) \\
\times\left[\frac{1+\left|\gamma_{p}^{(\alpha)}\right|^{2}}{1-\left|\gamma_{p}^{(\alpha)}\right|^{2}}+\frac{1}{\left(f_{p}^{(\alpha)}\right)^{2}} \sum_{s=0}^{\infty} \frac{1+\left|\Gamma_{p, s}^{(\alpha)}\right|^{2}}{\left(1-\left|\Gamma_{p, s}^{(\alpha)}\right|^{2}\right)^{2}}\right] \\
\stackrel{t_{\mathbf{L}}>>1, v=3 / 2}{\longrightarrow} \frac{p^{3}}{2 \pi^{2}} H^{2} \mathcal{Q}\left(p, \frac{v}{v}=3 / 2\right) \exp (-2 \alpha) \\
\times\left[\frac{1+\left|\gamma_{p}^{(\alpha)}\right|^{2}}{1-\left|\gamma_{p}^{(\alpha)}\right|^{2}}+\frac{1}{\left(f_{p}^{(\alpha)}\right)^{2}} \sum_{s=0}^{\infty} \frac{1+\left|\Gamma_{p, s}^{(\alpha)}\right|^{2}}{\left(1-\left|\Gamma_{p, s}^{(\alpha)}\right|^{2}\right)^{2}}\right] .
\end{gathered}
$$

Like the result in the case of field operator expansion method derived in the previous section, this result also implies that in the massless case $(v=3 / 2)$ amplitude of the vacuum fluctuation gets frozen with respect to the time scale when the associated modes exit horizon.

Further to know the exact wave number dependence of the amplitude of the normalised power spectrum from generalised $\alpha$ vacua we need to know the behaviour of the power spectrum at very short wavelengths $\left(p, p_{n}>>1\right)$. In this limit it is expected that the power spectrum of axion should match with the result obtained for spatially flat universe. In the short wave length approximation the time independent function $\mathcal{Q}(p>>1, \alpha, v)$ for any arbitrary mass parameter $v$ can be expressed for generalised $\alpha$ vacua as: 


$$
\begin{aligned}
\mathcal{Q} & (p \widetilde{>>1}, \alpha, v)=\frac{2^{2(\nu-1)}(\Gamma(v))^{2}}{p^{3} \pi} \mathcal{G}(\widetilde{p>>} 1) \\
& =\widetilde{\mathcal{M}(p, v)} \quad \forall \alpha
\end{aligned}
$$

where we have already defined the function $\mathcal{G}(\widetilde{p>>}$ ) in the earlier section. Here for very large wave number $p, p_{n}>>1$ one can write, $\mathcal{G}(\widetilde{p>>} 1) \sim 1+\cdots$, where all $\cdots$ are small correction terms. This also implies to the interesting fact that for large wavenumber limit and for any values of the parameter $\alpha$, the time independent function $\mathcal{Q}(p>>1, \alpha, v)$ computed for generalised $\alpha$ vacua exactly matches with the result obtained for Bunch Davies vacua in the earlier section i.e. $\mathcal{M}(\overrightarrow{p>>} 1, v)$. This means that the final result is independent of the choice of the parameter $\alpha$.

For the massless case $(v=3 / 2)$ in the short wave length approximation, the time independent function $\widetilde{\mathcal{Q}}(p>>$ $1, \alpha, v=3 / 2$ ) can further be simplified to:

$$
\begin{gathered}
\mathcal{Q}(p>>\widetilde{1, \alpha, v}=3 / 2)=\frac{\mathcal{G}(\widetilde{p>>} 1)}{2 p^{3}} \\
=\mathcal{M}(p>>1, v=3 / 2) \quad \forall \alpha .
\end{gathered}
$$

For the massless case $(v=3 / 2)$, in the same scale and the same approximation, the above amplitude takes the form:

$$
\begin{aligned}
\mathcal{P} & \left.p>>1, \alpha, t_{\mathbf{L}}>>1\right) \\
& =\frac{p^{3}}{2 \pi^{2}} \exp (-2 \alpha) H^{2} \mathcal{Q}(p>>\widetilde{1, \alpha, v}=3 / 2) \\
& =\frac{p^{3}}{2 \pi^{2}} \exp (-2 \alpha) H^{2} \mathcal{M}(p>>1, v \\
& =\left(\frac{H}{2 \pi}\right)^{2} \exp (-2 \alpha) \mathcal{G}(\widetilde{p>>}
\end{aligned}
$$

It is important to note that bo $h$ of Eqs (3.174) and (3.175)

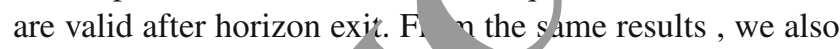
observe that the normaliso owt or cetrum from generalised $\alpha$ vacua,in the leadin order, nputed from reduced density matrix formalism is ctly sane as that obtained in the previous sub-section, comp d using field operator expansion method.

For comp we present the result for the two point correlation fun $\bar{r}$ and the associated power spectrum for Bunch vacuum by fixing the parameter $\alpha=0$ in our previou quations and they can be expressed as:

$$
\begin{aligned}
& \mathcal{B D}_{\mathbf{D}}\left(p>>1, t_{\mathbf{L}}>>1\right) \\
& =\frac{p^{3}}{2 \pi^{2}}\left(\cosh t_{\mathbf{L}}\right)^{2 v-3} H^{2} \mathcal{Q}(p>>1, \alpha=0, v) \\
& =\frac{p^{3}}{2 \pi^{2}}\left(\cosh t_{\mathbf{L}}\right)^{2 v-3} H^{2} \mathcal{M}(\widetilde{p>>} 1, v) \\
& =\left(2 \cosh t_{\mathbf{L}}\right)^{2 v-3}\left(\frac{H}{2 \pi}\right)^{2}\left(\frac{\Gamma(v)}{\Gamma\left(\frac{3}{2}\right)}\right)^{2} \mathcal{G}(\widetilde{p>>} 1) .
\end{aligned}
$$

For for the massless case $(v=3 / 2)$ this can be further simplified to:

$$
\begin{aligned}
\mathcal{P}_{\mathbf{B D}} & \left(p>>1, t_{\mathbf{L}}>>1\right) \\
& =\frac{p^{3}}{2 \pi^{2}} H^{2} \mathcal{Q}(p>>1, \widetilde{\alpha=} 0, v=3 / 2) \\
& =\frac{p^{3}}{2 \pi^{2}} H^{2} \mathcal{M}(p>>1, v=3 / 2) \\
& =\left(\frac{H}{2 \pi}\right)^{2} \mathcal{G}(\widetilde{p>c} 1) .
\end{aligned}
$$

In Fig. 9a, b we have shown the behaviour of the power spectrum of the mean square vacuum fluctuation computed from RDM formalism in the large wave number regime. We have considered $\alpha=0$ and $\alpha=0.1$ and fixed values of the mass parameter $v$ respectively. Additionally, in Fig. 9c we have depicted the behaviour of the power spectrum with respect to the mass parameter $v$ for fixed values of the parameter $\alpha=0,0.1,0.2,0.3,0.4$. From the figures, we observe 


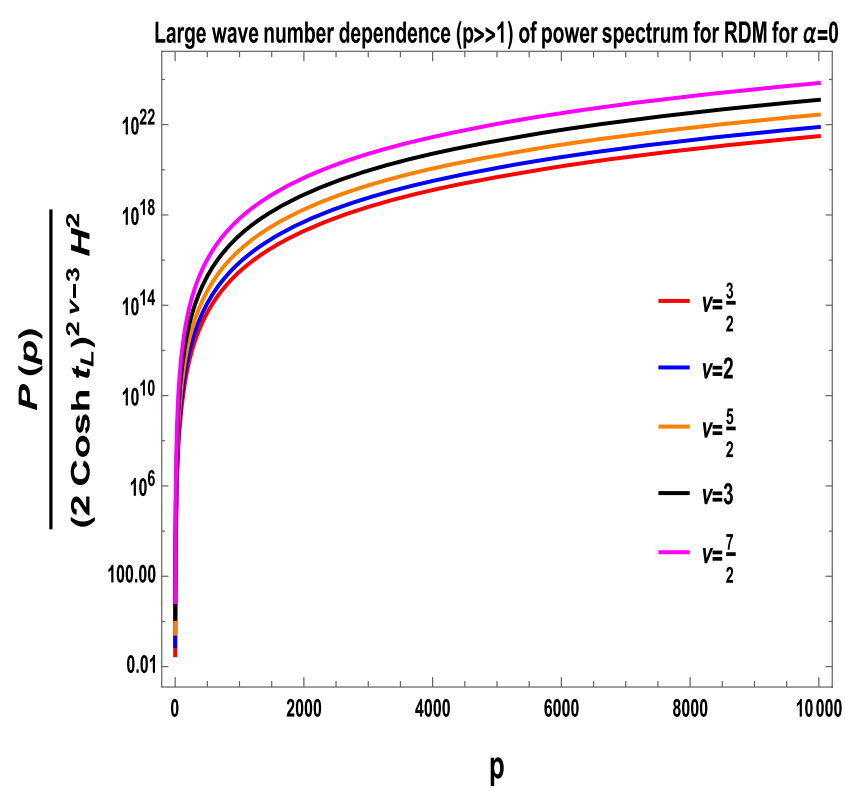

(a) Large wave number dependence of RDM power spectrum for $\alpha=0$.

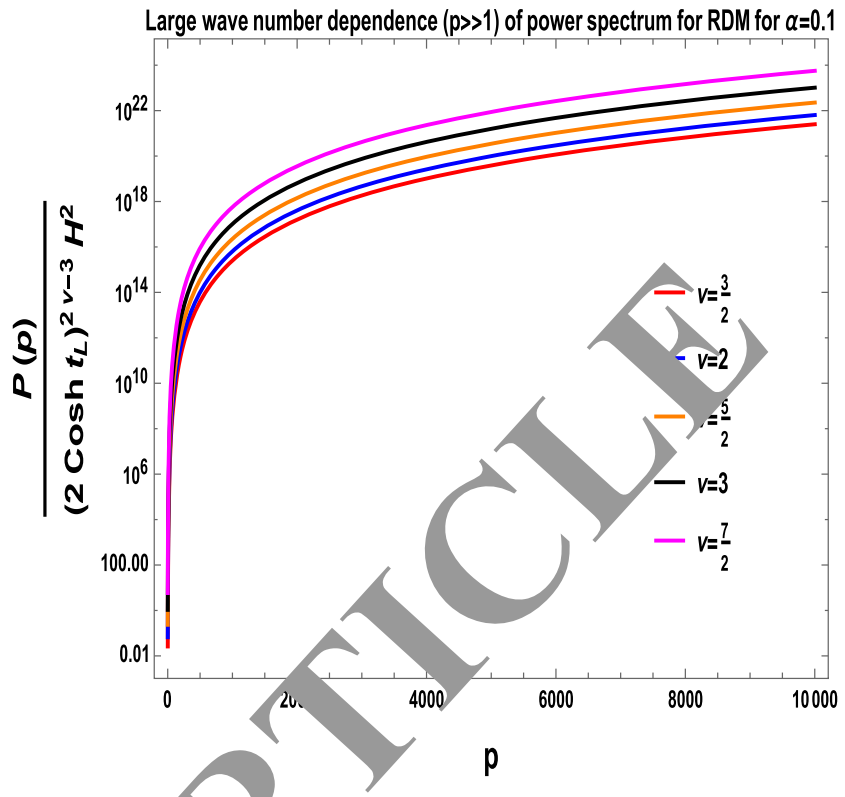

(b) number dependence of RDM power spectrum $\alpha=0.1$.

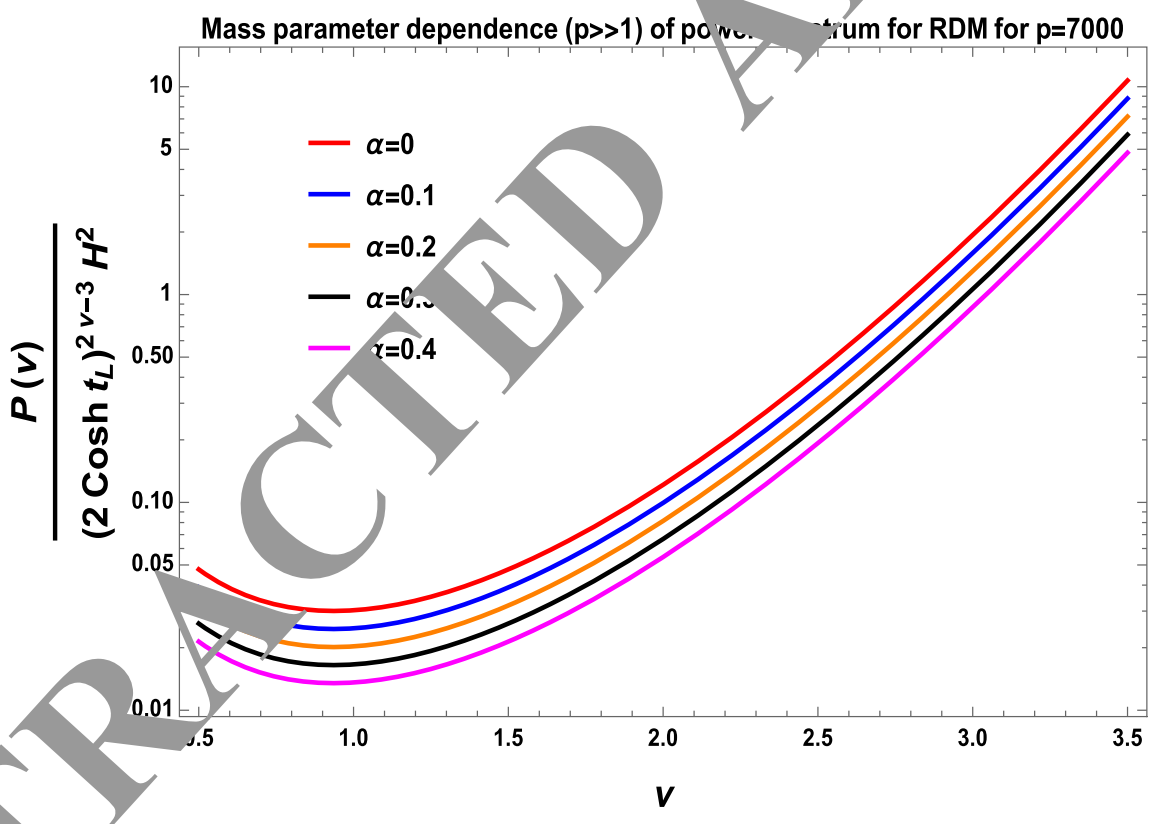

(c) Mass parameter dependence of RDM power spectrum in $p>>1$.

Fig. 9 E re of R' $\mathrm{R}$ power spectrum in large wave number region

that the power spectrum shows two distinctive behaviour in $1 / 2<v<1$ and $v>1$ region. For $1 / 2<v<1$ region the amplitude of the power spectrum decrease to a certain value and just after $v=1$ it increases. Also note that in large wave number regime, the power spectrum obtained from RDM formalism behaves in the same as way as that obtained from FOE formalism in the previous section.
On the other hand, to know the exact wave number dependence of the amplitude of the normalised power spectrum from generalised $\alpha$ vacua in the long wave length approximation, we need to know the behaviour of the power spectrum for $p, p_{n}<<1$. In this regime we expect that the power spectrum of axion should match with the result obtained for spatially flat universe. The time independent function 
$\mathcal{Q}(p \widehat{<<1}, \alpha, v)$ for the mass parameter $v \neq 3 / 2$ can be expressed for generalised $\alpha$ vacua as:

$\mathcal{Q}(p \widetilde{<<1}, \alpha, v)=\frac{2^{2(v-1)}(\Gamma(v))^{2}}{p^{3} \pi} \mathcal{G}(\widetilde{p<<}) \quad \forall \alpha$,

where the function $\mathcal{G}(\widetilde{p<<})$ is defined for $v \neq q / 2^{2}$ as:
$\mathcal{Q}(p<\widetilde{1, \alpha, v}=3 / 2)$ can be further simplified as:

$\mathcal{Q}(p<\widehat{1, \alpha, v}=3 / 2)=\frac{\mathcal{G}(p<\widetilde{<1, v}=3 / 2)}{2 p^{3}} \forall \alpha$,

where the function $\mathcal{G}(\widetilde{p<<} 1)$ is defined fo $\mathcal{\nu}=3 / 2 \mathrm{as}^{3}$ :

$$
\begin{aligned}
& \mathcal{G}(\widetilde{p<<} 1)=\frac{\pi p}{2|\cos \pi \nu|\left|\Gamma\left(v+\frac{1}{2}\right)\right|^{2}} \frac{\left|1-\gamma_{p}^{(\alpha)} \tilde{m}_{\mathbf{L R}}\right|^{2}}{\left|1-\gamma_{p}^{(\alpha)} \tilde{m}_{\mathbf{L R}}\right|^{2}-\left|\tilde{m}_{\mathbf{R R}}\right|^{2}} \\
& \times\left\{1+\frac{\left|\tilde{m}_{\mathbf{R} \mathbf{R}}\right|^{2}+\left(1-\gamma_{p}^{(\alpha)} \tilde{m}_{\mathbf{L R}}\right)^{*} \tilde{m}_{\mathbf{R R}}+\left(1-\gamma_{p}^{(\alpha)} \tilde{m}_{\mathbf{L R}}\right) \tilde{m}_{\mathbf{R} \mathbf{R}}^{*}}{\left|1-\gamma_{p}^{(\alpha)} \tilde{m}_{\mathbf{L R}}\right|^{2}}\right. \\
& +\sum_{n=0}^{\infty} \sqrt{\frac{p_{n}}{p} \frac{\left|1-\gamma_{p}^{(\alpha)} \tilde{m}_{\mathbf{L R}}\right|^{2}-\left|\tilde{m}_{\mathbf{R R}}\right|^{2}}{\left|1-\Gamma_{p, n}^{(\alpha)} \tilde{m}_{\mathbf{L R}, n}\right|^{2}-\left|\tilde{m}_{\mathbf{R R}, n}\right|^{2}}} \frac{1}{\left|1-\gamma_{p}^{(\alpha)} \tilde{m}_{\mathbf{L R}}\right|^{2}}\left[\left(1-\gamma_{p}^{(\alpha)} \tilde{m}_{\mathbf{L R}}\right)\left(1-{ }_{l}^{(\alpha)} \tilde{m}_{\mathbf{L R}, n}\right)^{*}+\tilde{m}_{\mathbf{R R}} \tilde{m}_{\mathbf{R R}, n}^{*}\right. \\
& +\left(1-\gamma_{p}^{(\alpha)} \tilde{m}_{\mathbf{L R}}\right) \tilde{m}_{\mathbf{R R}, n}^{*}+\left(1-\Gamma_{p, n}^{(\alpha)} \tilde{m}_{\mathbf{L R}, n}\right) \tilde{m}_{\mathbf{R} \mathbf{R}}^{*}+\left(1-\gamma_{p}^{(\alpha)} \tilde{m}_{\mathbf{L R}}\right) \\
& +\sum_{n=0}^{\infty} \sum_{m=0}^{\infty} \sqrt{\frac{p_{n} p_{m}}{p^{2}} \frac{\left(\left|1-\gamma_{p}^{(\alpha)} \tilde{m}_{\mathbf{L R}}\right|^{2}-\left|\tilde{m}_{\mathbf{R R}}\right|^{2}\right)}{\left(\left|1-\Gamma_{p, n}^{(\alpha)} \tilde{m}_{\mathbf{L R}, n}\right|^{2}-\left|\tilde{m}_{\mathbf{R R}, n}\right|^{2}\right)\left(\left|1-\Gamma_{p, m}^{(\alpha)} \tilde{m}_{\mathbf{L R}, m}\right|^{2}-\left|\tilde{m}_{\mathbf{R R}, m}\right|^{2}\right)}} \\
& \times \frac{1}{\left|1-\gamma_{p}^{(\alpha)} \tilde{m}_{\mathbf{L R}}\right|^{2}}\left[\left(1-\Gamma_{p, n}^{(\alpha)} \tilde{m}_{\mathbf{L R}, n}\right)\left(1-\Gamma_{p, m}^{(\alpha)} \tilde{m}_{\mathbf{L k},}\right)^{*}+\tilde{m}_{\mathbf{R R}, n} \tilde{m}_{\mathbf{R} \mathbf{R}, m}^{*}\right. \\
& +\left(1-\Gamma_{p, n}^{(\alpha)} \tilde{m}_{\mathbf{L R}, n}\right) \tilde{m}_{\mathbf{R}, m}^{*}+\left(1-\Gamma_{p, n}^{(\alpha)} \tilde{m}_{\mathbf{R}, n}{ }{ }_{\mathbf{R R}, r}\right. \\
& \left.\left.+\left(1-\Gamma_{p, n}^{(\alpha)} \tilde{m}_{\mathbf{L R}, \mathbf{n}}\right)^{*} \tilde{m}_{\mathbf{R} \mathbf{R}, m}+\left(1-\Gamma_{p, m}{ }_{\mathbf{R}, n}\right) \tilde{m}_{\mathbf{R}, m}\right]\right\}
\end{aligned}
$$

Here for very small wave number $p, p<<1$ ghe can write,

$$
\begin{aligned}
\mathcal{G}(\widehat{p<<}) \sim & \frac{\pi p}{2|\cos \pi v|\left|\Gamma\left(v+\frac{1}{2}\right)\right|^{2}} \\
& \times \frac{\left.(\alpha) \tilde{m}_{\mathbf{L} / \mathbf{R}}\right|^{2}}{\mid 1-\gamma_{p}}[1+\cdots],
\end{aligned}
$$

where all $\cdots$ are sh. correction terms. For Bunch Davies vacuum ance we fix $\alpha=0$, we find that the function $\mathcal{G}(p<<$ iny $\mathrm{d}$ pends on the mass parameter $v$ for massive in fic

On le contrary, for the case where $v=n / 2$ (which also inc es the massless situation $v=3 / 2$ ) the expression $\mathcal{G}(\vec{p}<1)$ diverges due to the overall factor $1 /|\cos \pi \nu|$. But we can avoid such unwanted divergent contributions by rewriting all the expressions for $p, p_{n}<<1$ with $v=n / 2$ that we have mentioned earlier. In such a situation for the massless case the time independent function

\footnotetext{
${ }^{2}$ Here $q$ is any positive odd integer.
}

$$
\begin{aligned}
\mathcal{G}(p & <\widetilde{<1, v}=3 / 2) \\
= & \frac{\pi}{2}\left\{1+\frac{\left(1 \pm e^{i \theta} \pi p e^{-p \pi}\right)}{\left|1 \pm e^{i \theta} \pi p e^{-p \pi}\right|} \sum_{n=0}^{\infty} \frac{\left(1 \pm e^{-i \theta} \pi p_{n} e^{-p_{n} \pi}\right)}{\left|1 \pm e^{i \theta} \pi p_{n} e^{-p_{n} \pi}\right|}\right. \\
& \left.+\sum_{n=0}^{\infty} \sum_{m=0}^{\infty} \sqrt{\frac{\left(1 \pm e^{i \theta} \pi p_{n} e^{-p_{n} \pi}\right)}{\left|1 \pm e^{i \theta} \pi p_{n} e^{-p_{n} \pi}\right|} \frac{\left(1 \pm e^{-i \theta} \pi p_{m} e^{-p_{m} \pi}\right)}{\left|1 \pm e^{i \theta} \pi p_{m} e^{-p_{m} \pi}\right|}}\right\}
\end{aligned}
$$

Here for very small wave number $p, p_{n}<<1$ with $v \neq 3 / 2$ and $v=3 / 2$ one can write,

$\mathcal{G}(\widetilde{p<<} 1) \sim \frac{\pi}{2}[1+\cdots]$,

\footnotetext{
${ }^{3}$ Here it is important to note the expression for the time dependent function $\mathcal{G}(\overrightarrow{p<<} 1)$ for $v=q / 2$ (where $q$ is any positive odd integer) in all cases are same. The only difference is appearing in the expression for the power spectrum. For $v=3 / 2$ case the power spectrum is scale invariant exactly. But for the other values of $v=1 / 2,5 / 2,7 / 2, \ldots$ the power spectrum is not scale invariant and small deviation from the scale invariant feature can be observed easily.
} 
where all $\cdots$ are small correction terms. For Bunch Davies vacuum we get the same result as the function $\mathcal{G}(p<<1)$ for massless axion field $(v=3 / 2)$ is independent of the parameter $\alpha$.

Moreover, it is important to note that the following contribution appearing in the normalised power spectrum for massive $(v \neq 3 / 2)$ and massless $(v=3 / 2)$ axion field can be simplified in the small wave number limit as:

$$
\begin{gathered}
{\left[\frac{1+\left|\gamma_{p}^{(\alpha)}\right|^{2}}{1-\left|\gamma_{p}^{(\alpha)}\right|^{2}}+\frac{1}{\left(f_{p}^{(\alpha)}\right)^{2}} \sum_{s=0}^{\infty} \frac{1+\left|\Gamma_{p, s}^{(\alpha)}\right|^{2}}{\left(1-\left|\Gamma_{p, s}^{(\alpha)}\right|^{2}\right)^{2}}\right]} \\
\underset{p<<1}{\approx}\left[\frac{\frac{(\sqrt{\cos 2 \pi v+1} \pm \sqrt{\cos 2 \pi v+3})^{2}\left(\cosh ^{2} \alpha+\sinh ^{2} \alpha e^{2 \pi i v}\right)^{2}}{\left[\cosh ^{2} \alpha+\sinh ^{2} \alpha e^{2 i \pi v}+\sinh 2 \alpha \cos \pi v e^{i \pi v}\right]^{2}}+2}{\frac{(\sqrt{\cos 2 \pi v+1} \pm \sqrt{\cos 2 \pi v+3})^{2}\left(\cosh ^{2} \alpha+\sinh ^{2} \alpha e^{2 \pi i v}\right)^{2}}{\left[\cosh ^{2} \alpha+\sinh ^{2} \alpha e^{2 i \pi v}+\sinh 2 \alpha \cos \pi v e^{i \pi v}\right]^{2}}-2}\right. \\
+\frac{1+\left|\frac{\sqrt{2}\left[\cosh ^{2} \alpha+\sinh ^{2} \alpha e^{2 i \pi v}+\sinh 2 \alpha \cos \pi v e^{i \pi v}\right]}{(\sqrt{\cos 2 \pi v+1} \pm \sqrt{\cos 2 \pi v+3})\left(\cosh 2 \alpha+\sinh ^{2} \alpha e^{2 \pi i v}\right)}\right|^{2}}{\left(1-\left|\frac{\sqrt{2}\left[\cosh ^{2} \alpha+\sinh ^{2} \alpha e^{2 i \pi v}+\sinh ^{2} \alpha \cos ^{2} v e^{i \pi v}\right]}{(\sqrt{\cos 2 \pi v+1} \pm \sqrt{\cos 2 \pi v+3})\left(\cosh ^{2} \alpha+\sinh ^{2} \alpha e^{2 \pi i v}\right)}\right|^{2}\right)^{4}}
\end{gathered}
$$$$
\underbrace{\left(\sum_{s=0}^{\infty} 1\right)^{-1}}_{=0}]
$$

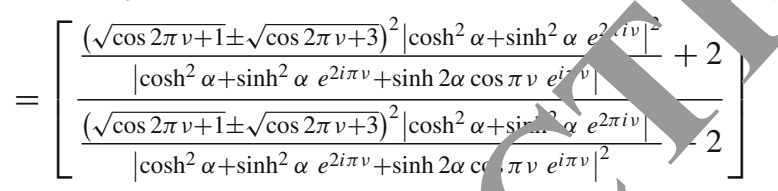$$
\forall \alpha \text { and } \nu \neq 3 / 2 \text {, }
$$$$
\left[\frac{1+\left|\gamma_{p}^{(\alpha, 3 / 2)}\right|^{2}}{1-\left|\gamma_{p}^{(\alpha, 3 / 2)}\right|^{2}}+\frac{1}{\left(f_{p}^{(\alpha, 3 / 2)}\right)^{2}} \Sigma_{s=1}^{\infty} 1+\frac{\left|\Gamma_{p, s}^{(\alpha, 3 / 2)}\right|^{2}}{\left.\left|\Gamma_{p, s}^{(\alpha, 3 / 2)}\right|^{2}\right)^{2}}\right]
$$

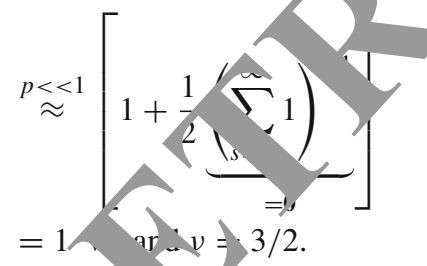

1. superhorizon time scales $\left(t_{\mathbf{L}}>>1\right)$ of region $\mathbf{L}$ the plitude of the normalised power spectrum of axion from generalised $\alpha$ vacua in the small wave number limit can be expressed as:

$$
\begin{aligned}
& \mathcal{P}\left(p<<1, \alpha, t_{\mathbf{L}}>>1\right) \\
& \quad=\frac{p^{3}}{2 \pi^{2}}\left(\cosh t_{\mathbf{L}}\right)^{2 v-3} \exp (-2 \alpha) H^{2} \mathcal{Q}(p \widetilde{<<1}, \alpha, v)
\end{aligned}
$$

$$
\begin{aligned}
& \times\left[\begin{array}{c}
\frac{(\sqrt{\cos 2 \pi \nu+1} \pm \sqrt{\cos 2 \pi \nu+3})^{2}\left|\cosh ^{2} \alpha+\sinh ^{2} \alpha e^{2 \pi i v}\right|^{2}}{\left|\cosh ^{2} \alpha+\sinh ^{2} \alpha e^{2 i \pi v}+\sinh 2 \alpha \cos \pi v e^{i \pi v}\right|^{2}}+2 \\
\frac{(\sqrt{\cos 2 \pi v+1} \pm \sqrt{\cos 2 \pi v+3})^{2}\left|\cosh ^{2} \alpha+\sinh ^{2} \alpha e^{2 \pi i v}\right|^{2}}{\left|\cosh ^{2} \alpha+\sinh ^{2} \alpha e^{2 i \pi v}+\sinh 2 \alpha \cos \pi v e^{i \pi v}\right|^{2}}-2
\end{array}\right] \\
& =\left(2 \cosh t_{\mathbf{L}}\right)^{2 v-3}\left(\frac{H}{2 \pi}\right)^{2}\left(\frac{\Gamma(v)}{\Gamma\left(\frac{3}{2}\right)}\right)^{2} \mathcal{G}(\widetilde{p<<} 1) \\
& \times\left[\frac{\frac{(\sqrt{\cos 2 \pi \nu+1} \pm \sqrt{\cos 2 \pi \nu+3})^{2} \mid \cosh ^{2} \alpha+\sinh ^{2}}{\mid \cosh ^{2} \alpha+\sinh ^{2} \alpha e^{2 i \pi \nu}+\sinh 2 \alpha \cos \pi v e^{i \pi}}+2}{\frac{(\sqrt{\cos 2 \pi v+1} \pm \sqrt{\cos 2 \pi v+3})^{2} \mid \cosh ^{2} \alpha}{\mid \cosh ^{2} \alpha+\sinh ^{2} \alpha e^{2 i \pi v}+\sinh 2 \alpha}-\frac{+\sinh ^{2} \alpha e^{2 \pi}}{v v e^{i \pi v} \mid}-2}\right] .
\end{aligned}
$$

For the massless case $(v=$ 2) in the superhorizon time scales $\left(t_{\mathbf{L}}>>1\right)$ of region the r. ritude of the normalised power spectrum of $x$ xion th generalised $\alpha$ vacua in the small wave number nit can be simplified in the present context as:

$$
\begin{aligned}
\mathcal{P} & (p<<1, \\
& =\frac{n^{3}}{2} T^{2} \operatorname{vn}(-2 \alpha) H^{2} \mathcal{Q}(p<\widetilde{1, \alpha}, v=3 / 2) \\
& =\left(\frac{1}{2 \pi}\right)^{2} \exp (-2 \alpha) \mathcal{G}(p<\widetilde{1, v}=3 / 2) .
\end{aligned}
$$

For Bunch Davies vacuum state $(\alpha=0)$, the mean square acuum fluctuation of axion can be expressed as:

$$
\begin{aligned}
\mathcal{P}_{\mathbf{B D}} & \left(p<<1, t_{\mathbf{L}}>>1\right) \\
= & \frac{p^{3}}{2 \pi^{2}}\left(\cosh t_{\mathbf{L}}\right)^{2 v-3} H^{2} \mathcal{Q}(p<\widehat{<1, \alpha}=0, v) \\
\times & {\left[\frac{(\sqrt{\cos 2 \pi v+1} \pm \sqrt{\cos 2 \pi v+3})^{2}+2}{(\sqrt{\cos 2 \pi v+1} \pm \sqrt{\cos 2 \pi v+3})^{2}-2}\right] } \\
= & \left(2 \cosh t_{\mathbf{L}}\right)^{2 v-3}\left(\frac{H}{2 \pi}\right)^{2}\left(\frac{\Gamma(v)}{\Gamma\left(\frac{3}{2}\right)}\right)^{2} \mathcal{G}(\widehat{p<<}) \\
\times & {\left[\frac{(\sqrt{\cos 2 \pi v+1} \pm \sqrt{\cos 2 \pi v+3})^{2}+2}{(\sqrt{\cos 2 \pi v+1} \pm \sqrt{\cos 2 \pi v+3})^{2}-2}\right] . }
\end{aligned}
$$

Also for the massless case $(v=3 / 2)$ in the superhorizon time scales $\left(t_{\mathbf{L}}>>1\right)$ of region $\mathbf{L}$ the amplitude of the normalised power spectrum of axion from Bunch Davies vacuum in the small wave number limit can be simplified as:

$$
\begin{aligned}
& \mathcal{P}_{\mathbf{B D}}\left(p<<1, t_{\mathbf{L}}>>1\right) \\
& =\frac{p^{3}}{2 \pi^{2}} H^{2} \mathcal{Q}(p<<1, \widetilde{\alpha=0}, v=3 / 2) \\
& =\left(\frac{H}{2 \pi}\right)^{2} \mathcal{G}(p<\widetilde{1, v}=3 / 2) .
\end{aligned}
$$






(a) Small wave number dependence of RDM power spectrum for $\alpha=0$ and $\nu=1,2,3,4,5$.

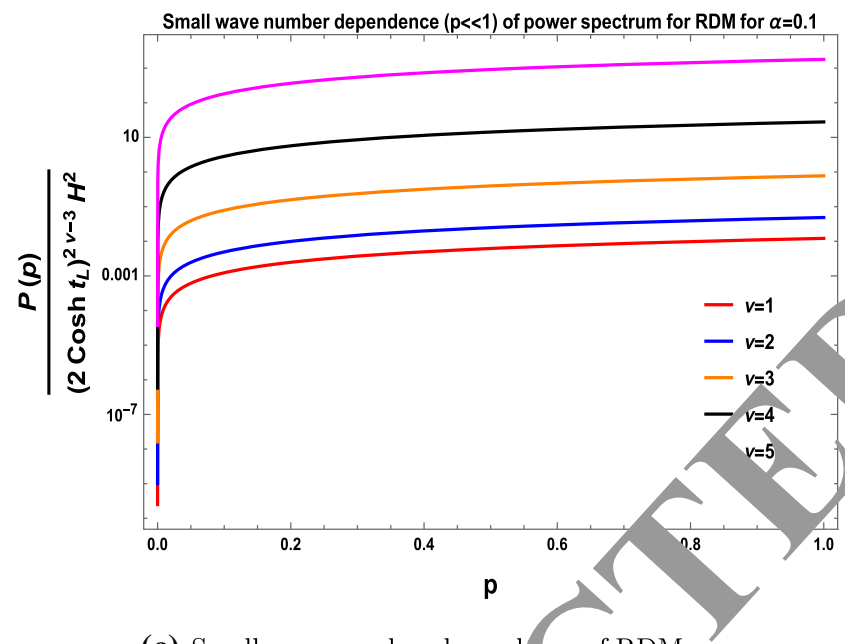

(c) Small wave number depend ace of RDM power spectrum for $\alpha=0.1$ and $\nu=1,2, \quad 4,5$.

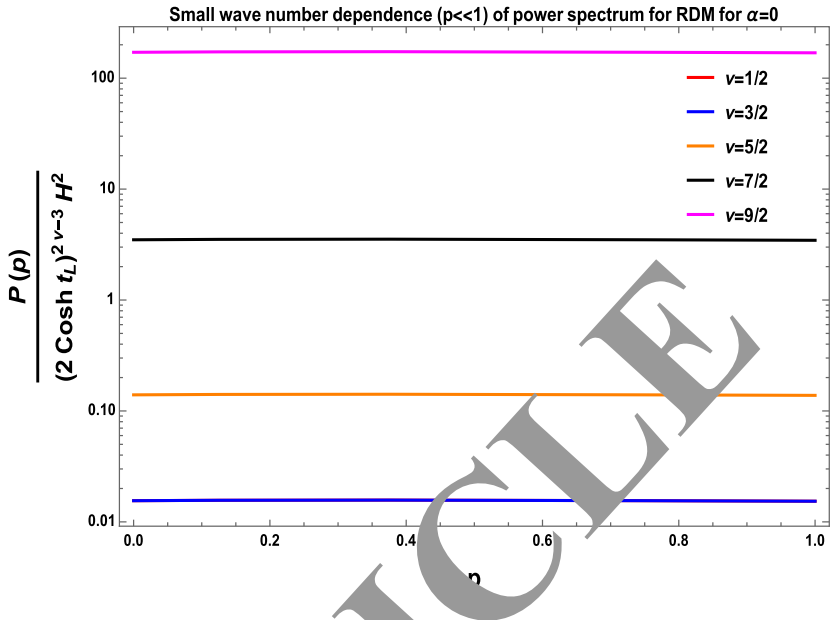

(b) Small way rumb lependence of RDM power spectrum for $\alpha$ and $\nu=/ 2,3 / 2,5 / 2,7 / 2,9 / 2$.

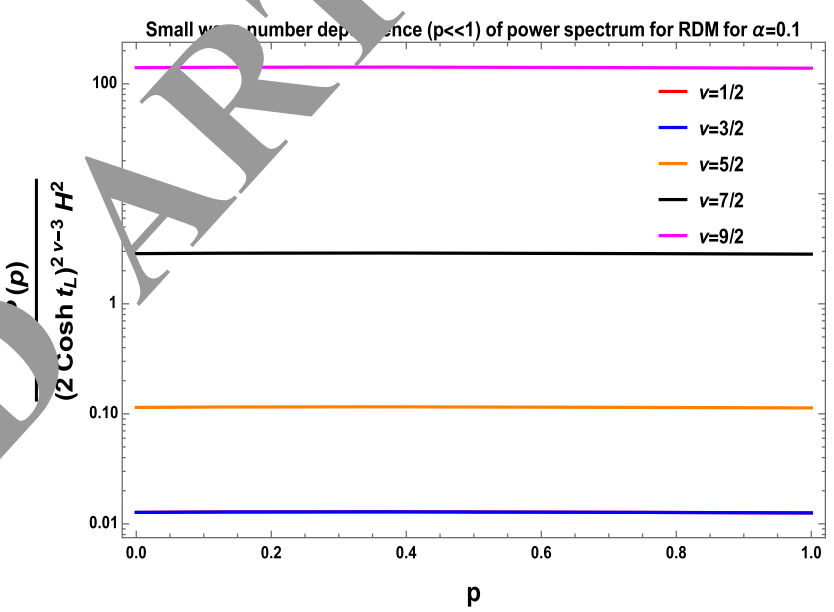

(d) Small wave number dependence of RDM power spectrum for $\alpha=0.1$ and $\nu=1 / 2,3 / 2,5 / 2,7 / 2,9 / 2$.

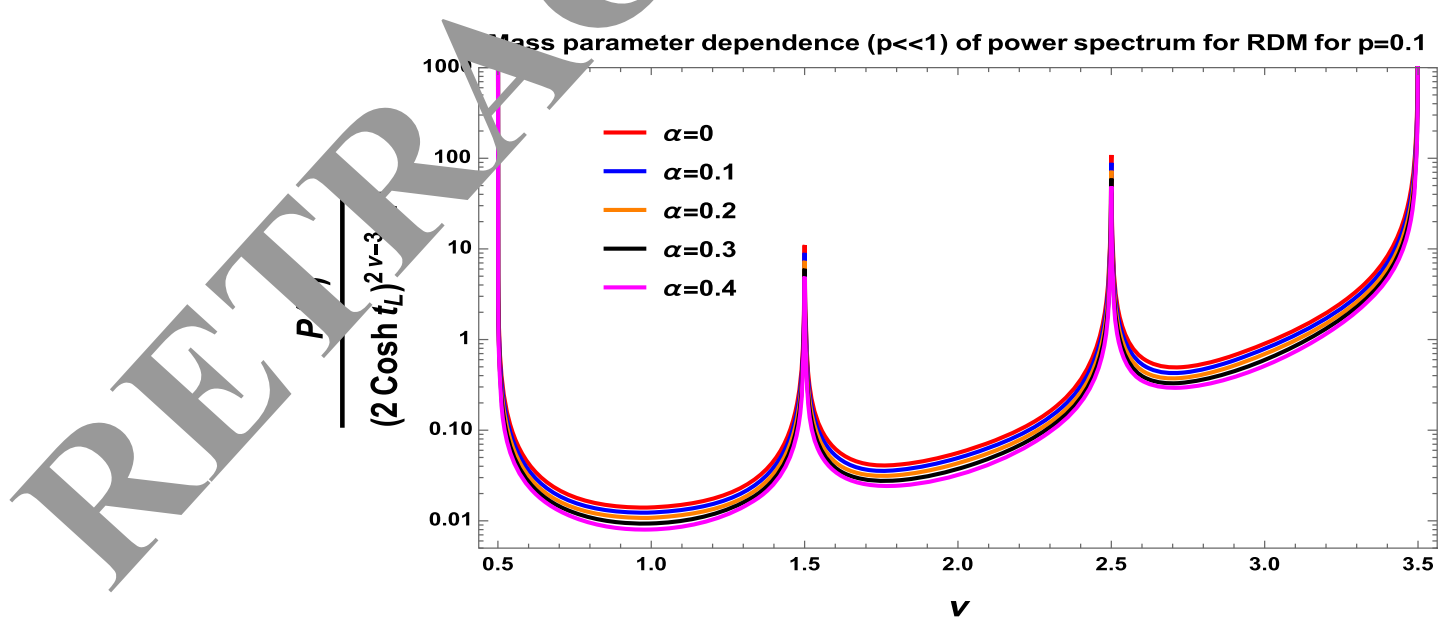

(e) Mass parameter dependence of RDM power spectrum in $p<<1$.

Fig. 10 Features of RDM power spectrum in small wave number region 
In Fig. 10a, c we have shown the behaviour of the power spectrum of the mean square vacuum fluctuation computed from RDM formalism in the small wave number regime for $\alpha=0$ and $\alpha=0.1$ and for fixed values of the mass parameter $v=1,2,3,3,4,5$ respectively. Moreover, in Fig. 10e we have presented the behaviour of the power spectrum with respect to the mass parameter $v$ with fixed values of the parameter $\alpha=0,0.1,0.2,0.3,0.4$. For the mass parameter dependence here we get distinctive feature for RDM formalism compared to FOE formalism which we discussed in the last subsection and the NES formalism which we discuss in the next subsection. From the plot, it is observed that for $v=1 / 2,3 / 2,5 / 2,7 / 2$ we get distinctive sharp peaks with constant and different magnitudes. On the other hand, in Fig. 10b, d we have shown the behaviour of the power spectrum in the small wave number regime for $\alpha=0$ and $\alpha=0.1$ with the fixed values of the mass parameter $v=1 / 2,3 / 2,5 / 2,7 / 2,9 / 2$. Here as the power spectrum is independent of the wave number, we get constant magnitude for different values of the mass parameter $v$.

3.3 Quantum vacuum fluctuation with non entangled state (NES)

In this subsection, we describe the quantum vacuum fluctua tion and its cosmological consequences using non ertangled state (NES) formalism. In this formalism we assum the wave function of the full de Sitter universe is de scribed region $\mathbf{L}$. So we do not use anyt informatior th the region R. In Fig. 11 we have presented a schema ic diag for the computation algorithm of NES forma' sm for non entangled quantum state of axion in de Sitter hy rbolic open chart.

\subsubsection{Non entangled state (NES) formo,ism}

In the region $\mathbf{L}$ the anction of the universe is described by the ion ent. led state (NES) and for generalised $\alpha$ vacua it iven by.

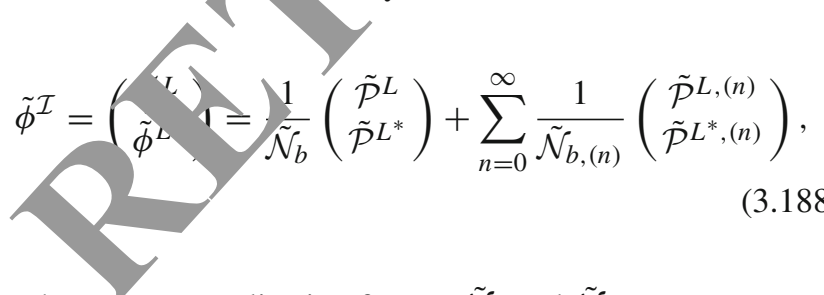

where the normalisation factors $\tilde{\mathcal{N}}_{b}$ and $\tilde{\mathcal{N}}_{b,(n)}$ are:

$$
\begin{aligned}
\tilde{\mathcal{N}}_{b} & =\frac{\sqrt{2 p}}{|\Gamma(1+i p)|}, \\
\tilde{\mathcal{N}}_{b,(n)} & =\frac{\sqrt{2 p_{n}}}{\left|\Gamma\left(1+i p_{n}\right)\right|} .
\end{aligned}
$$

We can also express the total wave function of the universe in terms of the oscillator mode expansion as given by:

$\tilde{\phi}^{L}\left(t_{\mathbf{L}}\right)=\frac{H}{\sinh t_{\mathbf{L}}}\left[b_{\mathcal{I}} \tilde{\phi}^{\mathcal{I}}\left(t_{\mathbf{L}}\right)+\sum_{n=0}^{\infty} b_{\mathcal{I},(n)} \tilde{\phi}_{(n)}^{\mathcal{I}}\left(t_{\mathbf{L}}\right)\right]$.

\subsubsection{Two point correlation function}

Using the above wave function we an further derive the mean square vacuum fluctuation hrou the ollowing two point correlation function:

$$
\begin{aligned}
& \left\langle\mathbf{L}\left|\tilde{\phi}_{p l m}^{L} \tilde{\phi}_{p^{\prime} l^{\prime} m^{\prime}}^{\dagger L}\right| \mathbf{L}\right\rangle=\left.\frac{H}{m^{2} m^{2} t_{\mathbf{L}}} \tilde{\mathrm{h}}^{L}\right|^{2} \exp (-2 \alpha) \delta\left(p-p^{\prime}\right) \delta_{l l^{\prime}} \delta_{m m^{\prime}} \\
& =P\left(p, \alpha, t_{\mathbf{L}}\right) \delta(\rho-192)
\end{aligned}
$$

where $P\left(p \alpha, t_{1}\right)$ is the power spectrum for non entangled state involvi crimudised $\alpha$ vacua. We can also define the normalised pow spectrum for non entangled state as:

$$
\begin{aligned}
\mathcal{P}\left(p, \alpha, t_{\mathbf{L}}\right) & =\frac{p^{3}}{2 \pi^{2}} P\left(p, \alpha, t_{\mathbf{L}}\right) \\
& =\frac{p^{3}}{2 \pi^{2}} \frac{H^{2}}{\sinh ^{2} t_{\mathbf{L}}}\left|\tilde{\phi}^{L}\right|^{2} \exp (-2 \alpha) .
\end{aligned}
$$

To quantify the normalised power spectrum for non entangled state, it is crcial to derive the expression for the square of the magnitude of the total wave function of the universe in the region $\mathbf{L}$, which is given by:

$$
\begin{aligned}
\left|\tilde{\phi}^{L}\right|^{2}= & \frac{1}{\left|\tilde{\mathcal{N}}_{b}\right|^{2}} \tilde{\mathcal{P}}^{L *} \tilde{\mathcal{P}}^{L}+\sum_{n=0}^{\infty} \frac{1}{\mathcal{N}_{b} \mathcal{N}_{b,(n)}^{*}}\left(\tilde{\mathcal{P}}_{(n)}^{L *} \tilde{\mathcal{P}}^{L}+\tilde{\mathcal{P}}^{L *} \tilde{\mathcal{P}}_{(n)}^{L}\right) \\
& +\sum_{n=0}^{\infty} \frac{1}{\mathcal{\mathcal { N }}_{b}^{*} \mathcal{N}_{b,(n)}}\left(\tilde{\mathcal{P}}_{(n)}^{L *} \tilde{\mathcal{P}}^{L}+\tilde{\mathcal{P}}^{L *} \tilde{\mathcal{P}}_{(n)}^{L}\right) \\
& +\sum_{n=0}^{\infty} \sum_{m=0}^{\infty} \frac{1}{\mathcal{N}_{b,(m)} \mathcal{N}_{b,(n)}^{*}}\left(\tilde{\mathcal{P}}_{(n)}^{L *} \tilde{\mathcal{P}}_{(m)}^{L}+\tilde{\mathcal{P}}_{(m)}^{L *} \tilde{\mathcal{P}}_{(n)}^{L}\right) .
\end{aligned}
$$

Further substituting the expressions for the normalisation factors, the above equation can be recast as:

$$
\begin{aligned}
\left|\tilde{\phi}^{L}\right|^{2}= & \frac{1}{2 p}|\Gamma(1+i p)|^{2} \tilde{\mathcal{P}}^{L *} \tilde{\mathcal{P}}^{L} \\
& +\sum_{n=0}^{\infty} \frac{1}{\sqrt{4 p p_{n}}}|\Gamma(1+i p)|\left|\Gamma\left(1-i p_{n}\right)\right| \\
& \times\left(\tilde{\mathcal{P}}_{(n)}^{L *} \tilde{\mathcal{P}}^{L}+\tilde{\mathcal{P}}^{L *} \tilde{\mathcal{P}}_{(n)}^{L}\right) \\
& +\sum_{n=0}^{\infty} \frac{1}{4 \sqrt{p p_{n}}}|\Gamma(1-i p)|\left|\Gamma\left(1+i p_{n}\right)\right|
\end{aligned}
$$




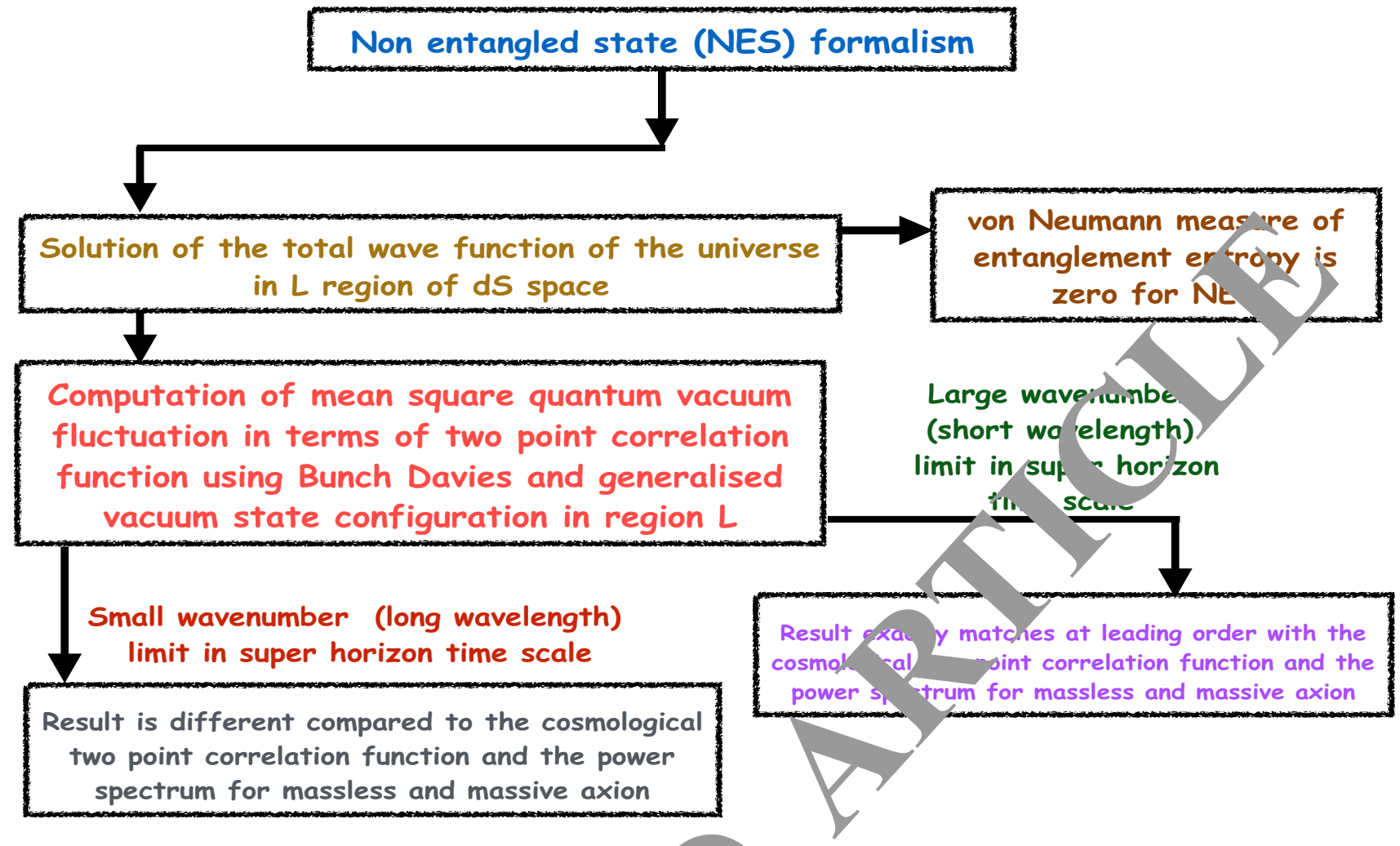

Fig. 11 Schematic diagram for the computation algorithm of NES Orm $_{\mathrm{t}}$ n for non entangled quantum state of axion in de Sitter hyperbolic open chart

$$
\begin{aligned}
& \times\left(\tilde{\mathcal{P}}_{(n)}^{L *} \tilde{\mathcal{P}}^{L}+\tilde{\mathcal{P}}^{L *} \tilde{\mathcal{P}}_{(n)}^{L}\right) \\
& +\sum_{n=0}^{\infty} \sum_{m=0}^{\infty} \frac{1}{\sqrt{4 p_{n} p_{m}}}\left|\Gamma\left(1-i p_{n}\right)\right|\left|\left(1-{ }_{m}\right)\right| \\
& \times\left(\tilde{\mathcal{P}}_{(n)}^{L *} \tilde{\mathcal{P}}_{(m)}^{L}+\tilde{\mathcal{P}}_{(m)}^{L *} \tilde{\mathcal{P}}_{(n)}^{L}\right)
\end{aligned}
$$

Consequently, the normalised po soctrum for non entangled state with generalised $\alpha$ vact? can be written as:

$$
\begin{aligned}
\mathcal{P}\left(p, \alpha, t_{\mathbf{L}}\right)= & \frac{p^{3}}{2} \\
& \left.+\sum_{n=0}^{\infty} \tilde{\mathcal{P}}_{(n)}^{L *} \tilde{\mathcal{P}}^{L}+\tilde{\mathcal{P}}^{L *} \tilde{\mathcal{P}}_{(n)}^{L}\right) \\
& +\sum_{n=0}^{\infty} \frac{1}{4 \sqrt{\left.\sinh ^{2} t_{\mathbf{L}}\right)}|\Gamma(1+i p)|\left|\Gamma\left(1-i p_{n}\right)\right|} \\
& \times\left(\tilde{\mathcal{P}}_{(n)}^{L *} \tilde{\mathcal{P}}^{L}+\tilde{\mathcal{P}}^{L *} \tilde{\mathcal{P}}_{(n)}^{L}\right) \\
& +\sum_{n=0}^{\infty} \sum_{m=0}^{\infty} \frac{1}{4 \sqrt{p_{n} p_{m}}}|\Gamma(1+i p)|\left|\Gamma\left(1+i p_{n}\right)\right| \\
& \left.\times\left(\tilde{\mathcal{P}}_{(n)}^{L *} \tilde{\mathcal{P}}_{(m)}^{L}+\tilde{\mathcal{P}}_{(m)}^{L *} \tilde{\mathcal{P}}_{(n)}^{L}\right)\right] .
\end{aligned}
$$

However, to extract further physical information from Eq. (3.162) for cosmological predictions, we consider the superhorizon time scales $\left(t_{\mathbf{L}}>>1\right)$ of region $\mathbf{L}$. In this limit, the Legendre functions as appearing in the complementary part and the particular integral part of the time dependent solution can be approximated to the following simplified form:

$$
\begin{aligned}
&\left(\widetilde{\mathcal{P}}^{\mathbf{L}}, \widetilde{\mathcal{P}}^{\mathbf{L} *}\right) \equiv P_{\nu-\frac{1}{2}}^{ \pm i p}\left(\cosh t_{\mathbf{L}}\right) \\
& \stackrel{t_{\mathbf{L}}}{ }>>1 \frac{2^{\nu-\frac{1}{2}}\left(\cosh t_{\mathbf{L}}\right)^{\nu-\frac{1}{2}} \Gamma(v)}{\sqrt{\pi} \Gamma\left(v \mp i p+\frac{1}{2}\right)}, \\
&\left(\widetilde{\mathcal{P}}_{(n)}^{\mathbf{L}}, \widetilde{\mathcal{P}}_{(n)}^{\mathbf{L} *}\right) \equiv P_{\nu-\frac{1}{2}}^{ \pm i p_{n}}\left(\cosh t_{\mathbf{L}}\right) \\
& \stackrel{t_{\mathbf{L}}>>1}{\longrightarrow} \frac{2^{\nu-\frac{1}{2}}\left(\cosh t_{\mathbf{L}}\right)^{\nu-\frac{1}{2}} \Gamma(v)}{\sqrt{\pi} \Gamma\left(v \mp i p_{n}+\frac{1}{2}\right)} .
\end{aligned}
$$

Thus, in the superhorizon time scales $\left(t_{\mathbf{L}}>>1\right.$ ) of region $\mathbf{L}$, Eq. (3.195) can be further simplified as:

$\left|\tilde{\phi}^{\mathbf{L}}\right|^{2} \underset{\mathbf{L}>>1}{\longrightarrow} \widetilde{\mathcal{K}(p, \alpha, v)}\left(\cosh t_{\mathbf{L}}\right)^{2 v-1}$

where the time independent function $\widetilde{\mathcal{K}(p, \alpha, \nu)}$ for generalised $\alpha$ vacua is defined 
as:

$$
\begin{aligned}
& \widetilde{\mathcal{K}(p, \alpha, v)}=\frac{2^{2 v-1}(\Gamma(v))^{2}}{\pi} \times\left[\frac{|\Gamma(1+i p)|^{2}}{2 p\left|\Gamma\left(v+i p+\frac{1}{2}\right)\right|^{2}}\right. \\
& \quad+\sum_{n=0}^{\infty} \frac{|\Gamma(1-i p)|\left|\Gamma\left(1+i p_{n}\right)\right|+|\Gamma(1+i p)|\left|\Gamma\left(1-i p_{n}\right)\right|}{4 \sqrt{p p_{n}} \Gamma\left(v-i p+\frac{1}{2}\right) \Gamma\left(v+i p_{n}+\frac{1}{2}\right)} \\
& \left.\quad+\sum_{n=0}^{\infty} \sum_{m=0}^{\infty} \frac{\left|\Gamma\left(1-i p_{n}\right)\right|\left|\Gamma\left(1+i p_{m}\right)\right|+\left|\Gamma\left(1+i p_{n}\right)\right|\left|\Gamma\left(1-i p_{m}\right)\right|}{4 \sqrt{p_{n} p_{m}} \Gamma\left(v-i p_{n}+\frac{1}{2}\right) \Gamma\left(v+i p_{m}+\frac{1}{2}\right)}\right] .
\end{aligned}
$$

Also in the super horizon time scale $\left(t_{\mathbf{L}}>>1\right)$ we get the following simplification in the normalised power spectrum for non entangled state:

$$
\begin{gathered}
\mathcal{P}\left(p, \alpha, t_{\mathbf{L}}\right)=\frac{p^{3}}{2 \pi^{2}} \frac{H^{2}}{\sinh ^{2} t_{\mathbf{L}}}\left|\tilde{\phi}^{L}\right|^{2} \exp (-2 \alpha) \\
\stackrel{t_{\mathbf{L}}>>1 \frac{p^{3}}{2 \pi^{2}} \frac{\left(\cosh t_{\mathbf{L}}\right)^{2 \nu-1}}{\sinh ^{2} t_{\mathbf{L}}}}{H^{2} \widehat{\mathcal{K}(p, v)} \exp (-2 \alpha) .}
\end{gathered}
$$

In this limit, for the massless case $(v=3 / 2)$, the time dependent contribution can be approximated into the following simplified form:

$$
\left(\frac{\left(\cosh t_{\mathbf{L}}\right)^{2 v-1}}{\sinh ^{2} t_{\mathbf{L}}}\right)_{\nu=3 / 2} \stackrel{t_{\mathbf{L}}>>1}{\longrightarrow} 1 .
$$

This implies that for an arbitrary value of the nar one can write:

$$
\left(\frac{\left(\cosh t_{\mathbf{L}}\right)^{2 v-1}}{\sinh ^{2} t_{\mathbf{L}}}\right) \stackrel{t_{\mathbf{L}}>1}{\longrightarrow}\left(\cosh t_{\mathbf{L}}\right)^{2 v} \mathbf{3}^{.}
$$

Consequently, in the superhorizon tin cales $\left(t_{\mathbf{L}}>>1\right)$ of region $\mathbf{L}$ and for the massless coce $\left(v=\nu_{,}\right)$, the amplitude of the normalised power spectrum yexpressed as:

$$
\begin{aligned}
& \mathcal{P}\left(p, \alpha, t_{\mathbf{L}}\right)=\frac{p^{3}}{2 \pi^{2}}-\left.\frac{}{2+} \tilde{\phi}^{L}\right|^{2} \operatorname{xp}(-2 \alpha) \\
& \stackrel{\longrightarrow}{\stackrel{\longrightarrow}{\longrightarrow}, \nu=3 / 2} \frac{p^{3}}{2 \pi^{2}} \\
& \left.H^{2} \mathcal{K}, \rho, v=3 / 2\right) \exp (-2 \alpha) \text {. }
\end{aligned}
$$

Like our in taer red in the previous section, this result also imr that in massless case $(v=3 / 2)$, the amplitude 5 he.$- m$ fluctuation gets frozen with respect to the time scal hen the associated modes exit horizon.

Furt, er, to know the exact wavenumber dependence of the amplitude of the normalised power spectrum from generalised $\alpha$ vacua, we need to know the behaviour of the power spectrum at very short wavelengths $\left(p, p_{n}>>1\right)$. In this limit, it is expected that the power spectrum of axion in the non entangled case should match with the result obtained for spatially flat universe. The time independent function
$\mathcal{K}(\widetilde{p, \alpha, v)}$ in this limit and for arbitrary mass parameter $v$ can be expressed as:

$\mathcal{K}(p>>1, \alpha, v)=\frac{2^{2(v-1)}(\Gamma(v))^{2}}{p^{3} \pi} \mathcal{U}(\widetilde{p>>} 1) \quad \forall \alpha$

where the function $\mathcal{U}(\widetilde{p>>})$ is defined as

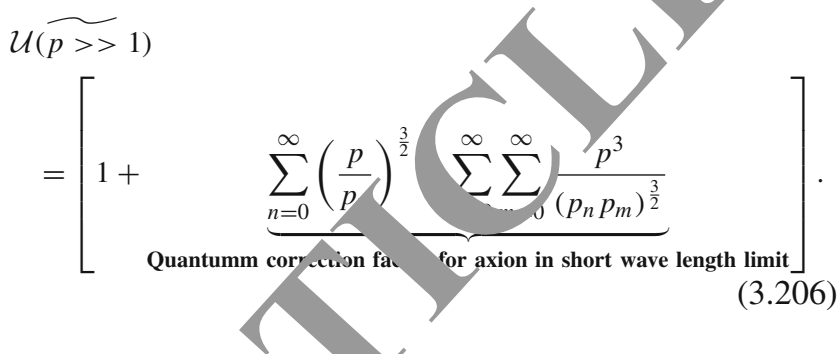

Thus, for ver rge way number $\left(p, p_{n}>>1\right)$, we can write, $\widetilde{\mathcal{U}(p} \sim 1$, , where all $\cdots$ are small correction terms. This an implies that for large wavenumber and for any va of the $m$ ass parameter $\alpha$, the time independent function $\mathcal{U}(\mathcal{P}, \varphi, \quad$, computed with generalised $\alpha$ vacua, matches with the result obtained for Bunch Davies vacua in the prerious subsection at the leading order in $\widehat{\mathcal{M}(p, v)}$. Also for . massless case $(v=3 / 2)$ the time independent function $\mathcal{K}, p, \alpha, v=3 / 2)$ in the short wave length limit can further oe simplified as:

$\mathcal{K}(p>>\widehat{1, \alpha, v}=3 / 2)=\frac{\mathcal{U}(\widetilde{p>>} 1)}{2 p^{3}} \forall \alpha$.

Finally, in the superhorizon time scales $\left(t_{\mathbf{L}}>>1\right)$ of region $\mathbf{L}$ the amplitude of the normalised power spectrum of axion from generalised $\alpha$ vacua for non entangled state in short wave length limit can be expressed as:

$$
\begin{aligned}
\mathcal{P} & \left(p>>1, \alpha, t_{\mathbf{L}}>>1\right) \\
= & \frac{p^{3}}{2 \pi^{2}}\left(\cosh t_{\mathbf{L}}\right)^{2 v-3} \exp (-2 \alpha) H^{2} \mathcal{K}(p \widetilde{>>1}, \alpha, v) \\
= & \left(2 \cosh t_{\mathbf{L}}\right)^{2 v-3}\left(\widehat{\frac{H}{2 \pi}}\right)^{2}\left(\frac{\Gamma(v)}{\Gamma\left(\frac{3}{2}\right)}\right)^{2} \\
& \times \exp (-2 \alpha) \mathcal{U}(\widetilde{p>>} 1) .
\end{aligned}
$$

For the massless case $(v=3 / 2)$ in the superhorizon time scales $\left(t_{\mathbf{L}}>>1\right)$ of region $\mathbf{L}$, the amplitude of the normalised power spectrum in short wave length limit can be simplified to:

$$
\begin{aligned}
\mathcal{P} & \left(p>>1, \alpha, t_{\mathbf{L}}>>1\right) \\
& =\frac{p^{3}}{2 \pi^{2}} \exp (-2 \alpha) H^{2} \mathcal{K}(p>>\widehat{1, \alpha, v}=3 / 2)
\end{aligned}
$$




$$
=\left(\frac{H}{2 \pi}\right)^{2} \exp (-2 \alpha) \mathcal{U}(\widetilde{p>>} 1) .
$$

Note that both the Eqs. (3.208) and (3.216) are valid after horizon exit. From these results we also observe that the power spectrum computed from non entangled state formalism is same, at the leading order approximation, as that computed from the FOE and RDM formalism, computed in earlier subsections. This is true in the large wavenumber limit of superhorizon time scale in region $\mathbf{L}$.

The result for the two point correlation function and the associated power spectrum for Bunch Davies vacuum can be obtained by setting $\alpha=0$ in the above equation and is found to be:

$$
\begin{aligned}
& \mathcal{P}_{\mathbf{B D}}\left(p>>1, t_{\mathbf{L}}>>1\right) \\
& =\frac{p^{3}}{2 \pi^{2}}\left(\cosh t_{\mathbf{L}}\right)^{2 v-3} H^{2} \mathcal{K}(p>>1, \alpha=0, v) \\
& =\left(2 \cosh t_{\mathbf{L}}\right)^{2 v-3}\left(\frac{H}{2 \pi}\right)^{2}\left(\frac{\Gamma(v)}{\Gamma\left(\frac{3}{2}\right)}\right)^{2} \mathcal{U}(\widetilde{p>>} 1) .
\end{aligned}
$$

For the massless case $(v=3 / 2)$ it reduces to:

$$
\begin{aligned}
& \mathcal{P}_{\mathbf{B D}}\left(p>>1, t_{\mathbf{L}}>>1\right) \\
& =\frac{p^{3}}{2 \pi^{2}} H^{2} \mathcal{K}(p>>1, \widetilde{\alpha=0}, v=3 / 2) \\
& =\left(\frac{H}{2 \pi}\right)^{2} \mathcal{U}(\widetilde{p>>} 1)
\end{aligned}
$$

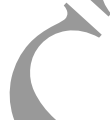

In Fig. 12a, b we have present spectrum of the mean square vacurm, uctuation computed inNES formalism for th - Ia $\mathrm{e}$ war number regime. This is shown for $\alpha=0$ and $\quad 0 \quad$ or fixed values of the mass parameter $v=3 / 2,2,5 / 2,7 / 2$ respectively. For both the values of $\alpha$, we g Imost s.milar behaviour. In Fig. 12c we have shown the behav rof the power spectrum with respect to the ma s parameter $v$ with fixed values of the parameter $\alpha=0,0 \quad 0.2,03,0.4$. Here for $1 / 2<v<1$ region and $1.0 n$ mass parameter dependence show two $d$ inc nozares. In $1 / 2<v<1$ region amplitude of the non ised power spectrum initially decrease and then just after $v)-1$ the amplitude of the power spectrum increase.

However, to examine the behaviour of the power spectrum in the long wavelength region and in the superhorizon time scale $\left(t_{\mathbf{L}}>>1\right)$, we take the limit $p<<1$. In the long wave length limit, the time independent function $\mathcal{K} \widetilde{(p, \alpha, v)}$ for any arbitrary mass parameter $v$ can be expressed (for $\alpha$ vacua) as:
$\mathcal{K}(p \widetilde{<<1}, \alpha, v)=\frac{2^{2(\nu-1)}(\Gamma(v))^{2}}{p \pi} \mathcal{U}(\widetilde{p<<1}) \quad \forall \alpha$,

where the function $\mathcal{U}(\widetilde{p<<})$ is given by:

$$
\begin{aligned}
& \mathcal{U}(\widetilde{p<<1)} \\
& =[1+\underbrace{\left(\frac{\left|\Gamma\left(v+\frac{1}{2}\right)\right|}{\Gamma\left(v+\frac{1}{2}\right)}\right)^{2}\left\{\sum_{n=0}^{\infty} \sqrt{\frac{p}{p_{n}}} \sum_{-0}^{\infty} \sum_{m=j}^{\infty} \frac{p}{\sqrt{p_{n} p_{m}}}\right\}}_{\text {Quantum correction facto for axion in lo }, \text { g wave length limit }} .
\end{aligned}
$$

For the massless case $(=-$, this can be further simplified to:

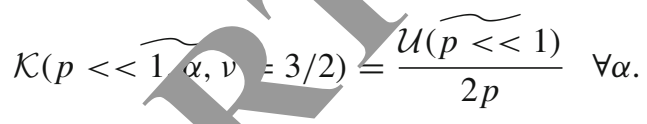

Moraver, in ,e superhorizon time scales $\left(t_{\mathbf{L}}>>1\right)$ of region t, Aplitude of the normalised power spectrum ( for $\alpha$ vacua ) for non entangled state (in the long wave length limit) can be expressed as:

$$
\begin{aligned}
\mathcal{P} & \left.p<<1, \alpha, t_{\mathbf{L}}>>1\right) \\
= & \frac{p^{3}}{2 \pi^{2}}\left(\cosh t_{\mathbf{L}}\right)^{2 v-3} \exp (-2 \alpha) H^{2} \mathcal{K}(p \widetilde{<<1}, \alpha, v) \\
= & \left(2 \cosh t_{\mathbf{L}}\right)^{2 v-3}\left(\frac{H}{2 \pi}\right)^{2} p^{2} \\
& \times \exp (-2 \alpha)\left(\frac{\Gamma(v)}{\Gamma\left(\frac{3}{2}\right)}\right)^{2} \mathcal{U}(\widetilde{p<<} 1)
\end{aligned}
$$

Also, for the massless case $(v=3 / 2)$, this reduces to:

$$
\begin{aligned}
\mathcal{P} & \left(p<<1, \alpha, t_{\mathbf{L}}>>1\right) \\
& =\frac{p^{3}}{2 \pi^{2}} \exp (-2 \alpha) H^{2} \mathcal{K}(p<\widetilde{1, \alpha, \nu}=3 / 2) \\
& =\left(\frac{H}{2 \pi}\right)^{2} p^{2} \exp (-2 \alpha) \mathcal{U}(\widetilde{p<<}) .
\end{aligned}
$$

The result for Bunch Davies vacuum is obtained by fixing $\alpha=0$ in above equation and is expressed as:

$$
\begin{aligned}
& \mathcal{P}_{\mathbf{B D}}\left(p<<1, t_{\mathbf{L}}>>1\right) \\
& =\frac{p^{3}}{2 \pi^{2}}\left(\cosh t_{\mathbf{L}}\right)^{2 v-3} H^{2} \mathcal{K}(p<\widetilde{<1, \alpha}=0, v) \\
& =\left(2 \cosh t_{\mathbf{L}}\right)^{2 v-3}\left(\frac{H}{2 \pi}\right)^{2} p^{2}\left(\frac{\Gamma(v)}{\Gamma\left(\frac{3}{2}\right)}\right)^{2} \mathcal{U}(\widetilde{p<<} 1)
\end{aligned}
$$




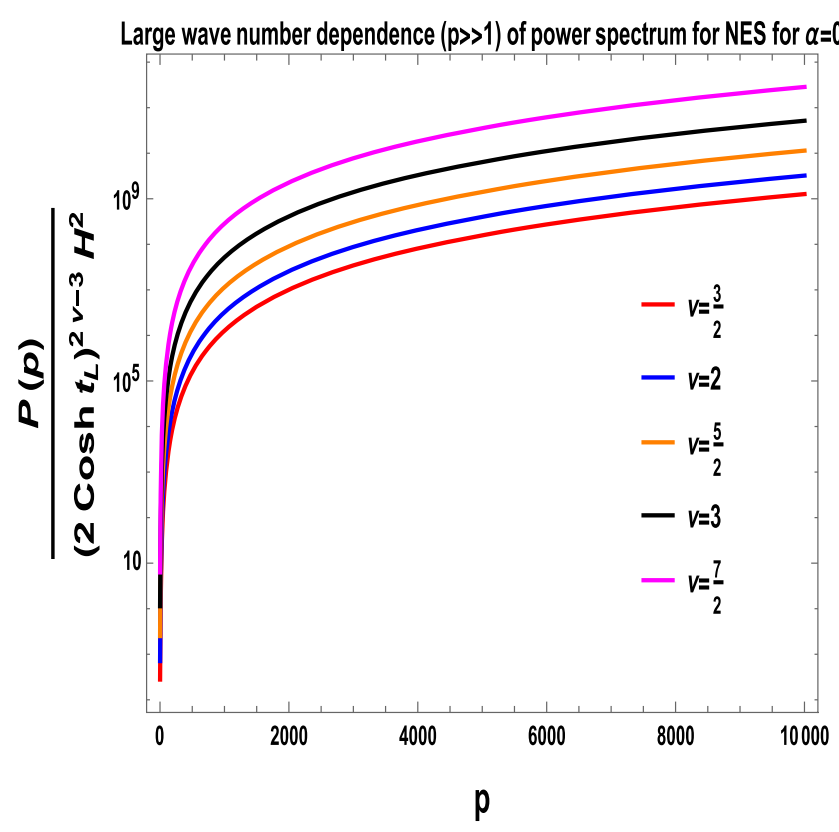

(a) Large wave number dependence of NES power spectrum for $\alpha=0$.



(b) Là wave number dependence of NES power spectrum for $=0.1$.



(c) Mass parameter dependence of NES power spectrum for $p>>1$.

Fig. 12 Fea of NES power spectrum in large wave number region

whi for the massless case $(v=3 / 2)$ reduces to:

$$
\begin{aligned}
& \mathcal{P}_{\mathbf{B D}}\left(p<<1, t_{\mathbf{L}}>>1\right) \\
& =\frac{p^{3}}{2 \pi^{2}} H^{2} \mathcal{K}(p<<1, \widehat{\alpha=0}, v=3 / 2) \\
& =\left(\frac{H}{2 \pi}\right)^{2} p^{2} \mathcal{U}(\widetilde{p<<}) .
\end{aligned}
$$

In Fig. 13a, b, we have shown the behaviour of the power spectrum of the mean square vacuum fluctuation in NES formalism in the small wave number regime for $\alpha=0$ and $\alpha=0.1$ with fixed values of the mass parameter $v=3 / 2,2,5 / 2,3,7 / 2$ respectively. Note that in both the cases we find almost similar behaviour. Also, in Fig. 13c we have shown the behaviour of the power spectrum with respect to the mass parameter $v$ with fixed values of $\alpha=$ 


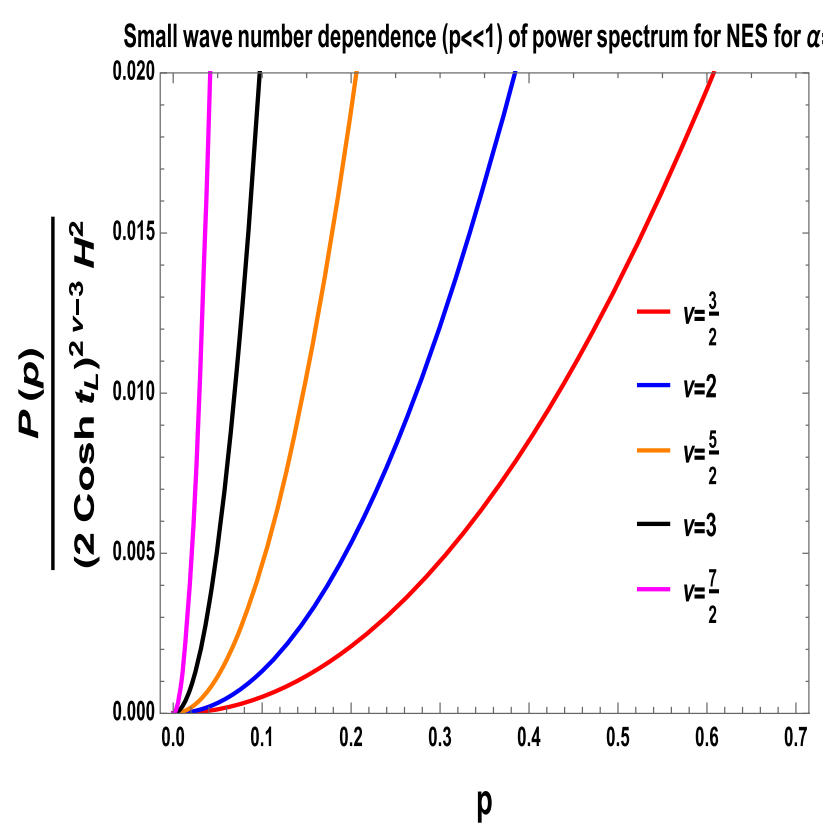

(a) Small wave number dependence of NES power spectrum for $\alpha=0$

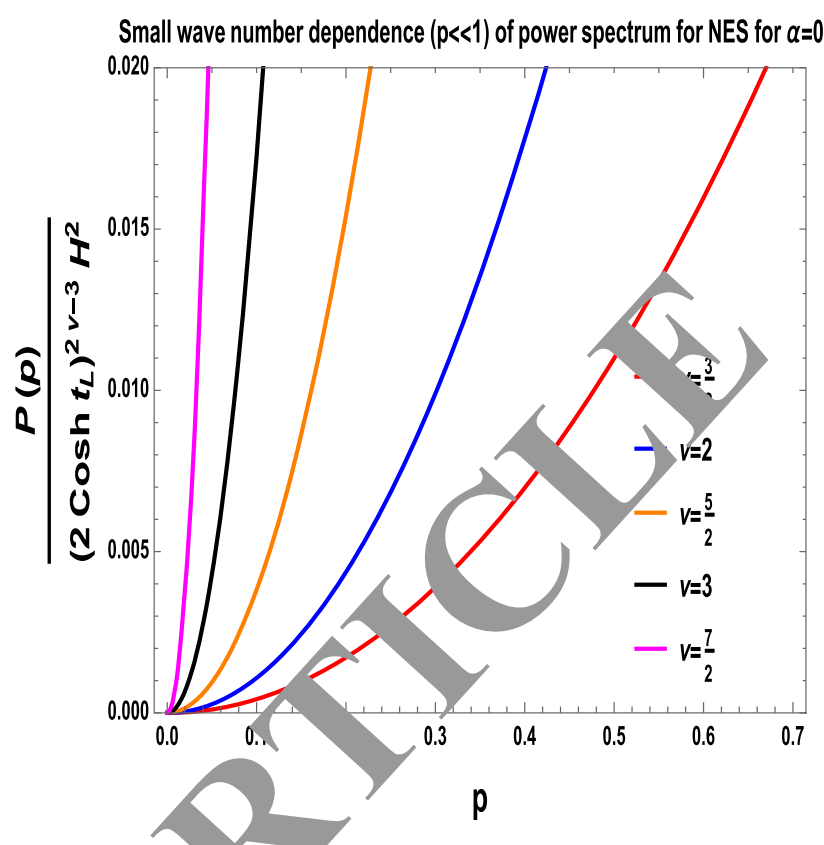

(b) Sn wave number dependence of NES power spectrum for $y=0.1$.

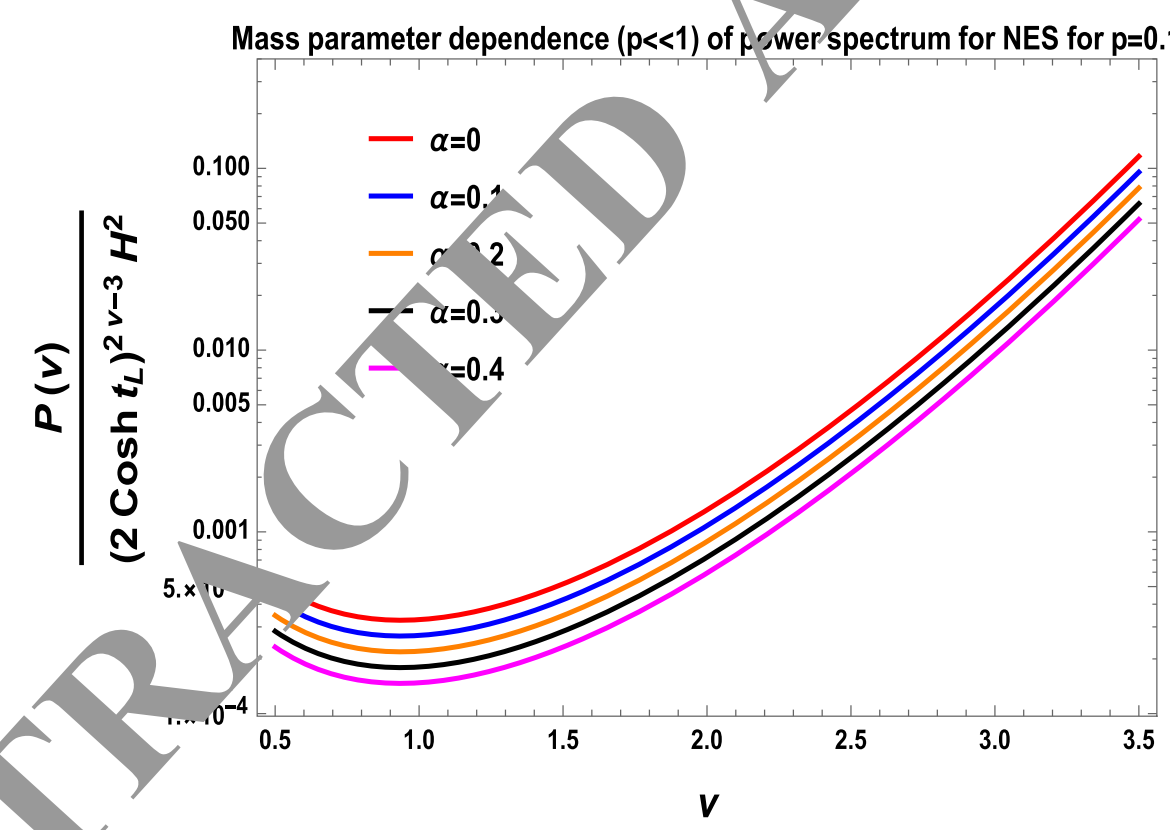

(c) Mass parameter dependence of NES power spectrum in $p<<1$.

Fig. 13 Feà of JES power spectrum in small wave number region

$0,0.7 .2,0.3,0.4$. In this case we again observe two distinct re ${ }_{j}$ ions of mass parameter dependence.

We have explicitly presented the comparison among FOE, RDM and NES formalism for $\alpha$ vacua in Table 1. The same table is valid for Bunch Davis vacuum when $\alpha=0$. We have quoted the differences, among the findings from these formalism, for the primordial power spectrum from mean square vacuum fluctuation at large and small scales.

\section{Summary}

To summarize, in this work, we have addressed the following issues:

- We have explicitly studied the power spectrum of mean squared vacuum fluctuation for axion field using the concept of quantum entanglement in de Sitter space. The 
Table 1 Comparison between FOE, RDM and NES formalism for $\alpha$ vacua

\begin{tabular}{|c|c|c|c|}
\hline Feuatures & FOE & RDM & NES \\
\hline Wave function & $\begin{array}{l}\text { Here we solve the wave function in } \\
\mathbf{L} \text { region of dS space }\end{array}$ & $\begin{array}{l}\text { Here we solve the wave function in } \\
\mathbf{L} \text { and } \mathbf{R} \text { region of dS space }\end{array}$ & $\begin{array}{l}\text { Here we only solve the wave } \\
\text { function in } \mathbf{L} \text { region of dS space }\end{array}$ \\
\hline Quantum state & $\begin{array}{l}\text { Here we deal with entangled } \\
\text { quantum state }\end{array}$ & $\begin{array}{l}\text { Here we deal with mixed quantum } \\
\text { state }\end{array}$ & $\begin{array}{l}\text { Here we deal with non-entangled } \\
\text { quantum state }\end{array}$ \\
\hline $\begin{array}{l}\text { Quantum number } \\
\text { dependence }\end{array}$ & $\begin{array}{l}\text { Power spectrum is only dependent } \\
\text { on } \mathbf{S O}(\mathbf{1 , 3}) \text { quantum number } \mathrm{p} \\
\text { and independent on } 1, \mathrm{~m}\end{array}$ & $\begin{array}{l}\text { Power spectrum is only dependent } \\
\text { on } \mathbf{S O}(\mathbf{1 , 3}) \text { quantum number } \mathrm{p} \\
\text { and independent on } 1, \mathrm{~m}\end{array}$ & $\begin{array}{l}\text { Power spectrum is only dependent } \\
\text { on } \mathbf{S O}(\mathbf{1 , 3}) \mathrm{qu} \text {. } \\
\text { and independent } \\
\mathrm{m}\end{array}$ \\
\hline Time scale for computation & $\begin{array}{l}\text { Analysis is performed on } \\
\text { superhorizon time scale }\end{array}$ & $\begin{array}{l}\text { Analysis is performed on } \\
\text { superhorizon time scale }\end{array}$ & $\begin{array}{l}\text { perforined on } \\
\text { n tim/scale }\end{array}$ \\
\hline $\begin{array}{l}\text { Power spectrum spectrum at } \\
\text { large wave number }\end{array}$ & $\begin{array}{l}\text { Leading order term is } \\
\left(\frac{H}{2 \pi}\right)^{2} \exp (-2 \alpha) \text { and the next } \\
\text { order effects are different from } \\
\text { RDM and NES for massless } \\
\text { axion }(v=3 / 2)\end{array}$ & $\begin{array}{l}\text { Leading order term is } \\
\left(\frac{H}{2 \pi}\right)^{2} \exp (-2 \alpha) \text { and the next } \\
\text { order effects are different from } \\
\text { FOE and NES for massless axic } \\
(v=3 / 2)\end{array}$ & $\begin{array}{l}\left.\frac{d i n g}{\pi}\right)^{2} \text { erde } p(-2 \alpha) \text { and the next } \\
\text { der ef ects are different from } \\
\text { ion }(\nu=3 / 2)\end{array}$ \\
\hline $\begin{array}{l}\text { Power spectrum at small at } \\
\text { small wave number }\end{array}$ & $\begin{array}{l}\text { Leading order term is } \\
\left(\frac{H}{2 \pi}\right)^{2} p^{3} \exp (-2 \alpha) \text { and the } \\
\text { next order effects are different } \\
\text { from RDM and NES for massless } \\
\text { axion }(v=3 / 2)\end{array}$ & $\begin{array}{l}\text { Leading order term is } \\
\frac{H^{2}}{8 \pi} \exp (-2 \alpha) \text { and the } \\
\text { effects are differe } 1 \mathrm{t} \\
\text { and NES for } \mathrm{m} \text { less } \\
(\nu=3 / 2)\end{array}$ & $\begin{array}{l}\text { Leading order term is } \\
\left(\frac{H}{2 \pi}\right)^{2} p^{2} \exp (-2 \alpha) \text { and the } \\
\text { next order effects are different } \\
\text { from FOE and RDM for massless } \\
\text { axion }(v=3 / 2)\end{array}$ \\
\hline
\end{tabular}

effective action for the axion field, used here, has its origin from Type IIB String theory compactified to four dimensions. . For our analysis, we have chosen ty 0 initial vacuum states i.e. Bunch Davies and a gene. alised class of $\alpha$ vacua. The power spectrum of mean squared vacuum fluctuation is computed using curstinctive formalisms: (1) Field operator exp nsion ( (2) Reduced density matrix (RDM) an $r^{\prime}(\alpha$ Von entangled state (NES). In all three cases, th comp. ion has been done starting with two oper charts in hyperbolic manifold of de Sitter space consi $n g$ of ty o regions: $\mathbf{L}$ and $\mathbf{R}$. Though the starting point is s. e construction of these three formalisms ai cfarent from each other and have their own physical sirrificance. Each of the formalism has been discus. $d$ in teve of the papers and some details of approxin io them are presented in the appendix. Simplarities differences from each other are present $a$ table.

- In case $f$ FOE 1 alism we solve for the wave function $\mathrm{j}$ the region $\boldsymbol{L}$ and using this solution we compute the ge. al exp ession for the mean square vacuum fluci. on at is quantum correction in terms of two point cor 1ntin function. The result is evaluated at all momenscales. We considered two limiting approximation in the naracteristic momentum scales, i.e. large wave number (small wave length in which the corresponding scale is smaller than the curvature radius of the de Sitter hyperbolic open chart) regime and small wave number (long wave length in which the corresponding scale is larger than the curvature radius of the de Sitter hyperbolic open chart) regime. We have observed distinctive features in the power spectrum of of mean squared vacuum fluctuation in these two different regimes. In the large wave number (small wave length) regime we found that the leading order result for the power spectrum is consistent with the known result for observed cosmological correlation function in the super horizon time scale. The correction to the leading order result that we computed for the power spectrum can be interpreted as the sub-leading effect in the observed cosmological power spectrum. This is a strong information from the perspective of cosmological observation since such effects, possibly due to quantum entanglement of states, can play a big role to break the degeneracy of the observed cosmological power spectrum in the small wave length regime. On the other hand, in the long wave length regime we found that the power spectrum follows completely different momentum dependence in the super horizon time scale. Since in this regime and in this time scale, at present, we lack adequate observational data on power spectrum we are unable to comment on our result with observation. But our result for the power spectrum in long wave length limit and super horizon time scale can be used as a theoretical probe to study the physical implications and its observational cosmological consequences in near future. Our result also implies that the mean square vacuum fluctuation for axion field, in super horizon time scale, gets enhanced in long wave length regime and freezes in the small wave length regime. We also observe that for a massive axion, the power spectrum is nearly scale invariant in all momentum scales. On the other hand, for massless axion we observe exact scale invariance only in large wave number (small wave 
length) regime and for the Bunch Davies initial quantum state. For generalised $\alpha$ initial state, we find slight modification in the corresponding power spectrum of the mean square vacuum fluctuation. The modification factor is proportional to $\exp (-2 \alpha)$ which is valid for all values of the parameter $\alpha$. It also implies that for large value of the parameter $\alpha$ we get additional exponential suppression for the power spectrum. This information can be used to distinguish between the role of Bunch Davies vacuum $(\alpha=0)$ and any $\alpha$ vacua quantum initial state during analysis of observational data.

- In RDM formalism, the wave function for the axion field is solved in $\mathbf{L}$ and $\mathbf{R}$ regions of the de Sitter open chart. This solution has been used to compute the mean square vacuum fluctuation and its quantum correction for both Bunch Davies and $\alpha$ vacuum state. Corresponding results are evaluated at all momentum scales by partially tracing out all the information from the region $\mathbf{R}$. Like in the case of FOE, we considered the small and large wavelength approximations in the characteristic momentum scales and found distinct features in the corresponding power spectrum. In the small wave length regime again the leading order result, in super horizon time scales matched with known result (same as FOE). However, the sub-leading order result for the power spectrum is diff ent from the result obtained from FOE formalism whic distinguishes the two approaches. Moreover, in the long wave length regime the power spectrum has $\mathrm{CO}_{1} 1_{-\mathrm{wly}}$ different momentum dependence compare to $\mathrm{FO}_{\mathrm{L}}$ malism. We also notice that the enhan en t of mean square vacuum fluctuation for axion eld, in $\%$ wave length regime, is different (slowe) in nature compared to FOE formalism but the freezir in shor wavelength regime is of same nature. The obser on scale invariance of power spectrum in th alism remains similar to that in FOE formalism.

- In the last formalisn 1.0 IES, ti $\%$ wave function of axion field is solved in th of the de Sitter hyperbolic open chart. With the $1 \mathrm{n}$ of this solution, $t$ we computed the $\mathrm{r}$ ea quare vacuum fluctuation using Bunch Davies nd $\alpha$ va $m$ state configuration. The correspon' 'ng result is evaluated at all momentum scales. Like the $\mathrm{p}$ us $\mathrm{t}$ ro cases, here also we reverted to two ii. ing a oximations i.e. large wave number (small wa noth ) regime and small wave number (long wave th) regime. We again observed distinctive behaviour in $\mathrm{t}$, e power spectrum in these two different regimes. In the large wave number (small wave length) regime, the leading order result for power spectrum matches with the known result for observed cosmological correlation function just as the cases of FOE and RDM formalism. However, the sub-leading order result s completely different FOE as well as RDM formalism. Thus, it is the sub-leading terms which distinguish these formalisms from each other and they can be confronted with future observational data. On the other hand, in the small wave number (long wave length) regime, even the leading order result for the power spectrum differs, in momentum dependence, compared to the result obtained from FOE and RDM formalism. Also the nature of shancement of the mean square vacuum fluctuation $\mathrm{T} E$ for nalism is found to be different from that in FOE . $\mathrm{R} D \mathrm{M}$ formalism but the nature of freezin and the obs_rvation on scale invariance of power spectru emai.is same in all the three cases.

- For completeness, we dis uss the ictual reason for the results obtained for he p ar pectra from quantum entangled state as ppe $\mathrm{ng}$ in FOE formalism and the mixed state wh is used $y$ construct the RDM formalism. To do so, wo onsider two subsystems, $L$ and $R$ using whin ne can onstruct the quantum mechanical state $y$ or o mion field as $|\Psi\rangle$ axion. In our computation, these bsystems are defined in the region $\mathbf{L}$ and $\mathbf{R}$ re atively 1,1 the de Sitter hyperbolic open chart. Now using state vector of axion field we can define the density matrix as:

$\rho_{\text {axion }}=\left|\Psi_{\text {axion }}\right\rangle\left\langle\Psi_{\text {axion }}\right|$,

in both the subsystems, $L$ and $R$ for FOE and RDM formalism and only the system $L$ for NES formalism. Using this density matrix we can express the expectation value (for the total system) of a quantum mechanical operator

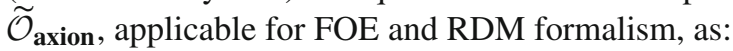

$$
\begin{aligned}
\operatorname{Tr} & \left(\rho_{\text {axion }} \widetilde{\mathcal{O}}_{\text {axion }}\right) \\
& =\sum_{\mathbf{L}} \sum_{\mathbf{R}}\left\langle\mathbf{L}, \mathbf{R} \mid \Psi_{\text {axion }}\right\rangle\left\langle\Psi_{\text {axion }}\left|\widetilde{\mathcal{O}}_{\text {axion }}\right| \mathbf{L}, \mathbf{R}\right\rangle \\
& \equiv\left\langle\Psi_{\text {axion }}\left|\widetilde{\mathcal{O}}_{\text {axion }}\right| \Psi_{\text {axion }}\right\rangle \\
& \equiv\left\langle\widetilde{\mathcal{O}}_{\text {axion }}\right\rangle .
\end{aligned}
$$

This is an important observation as it is related to the measurement and quantification of any physical cosmological observable in the quantum regime. But in the case of NES formalism one can rewrite Eq. (4.2) as:

$$
\begin{aligned}
\operatorname{Tr} & \left(\rho_{\text {axion }} \widetilde{\mathcal{O}}_{\text {axion }}\right) \\
= & \sum_{\mathbf{L}} \sum_{\mathbf{R}}\left\langle\mathbf{L}, \mathbf{R} \mid \Psi_{\text {axion }}\right\rangle\left\langle\Psi_{\text {axion }}\left|\widetilde{\mathcal{O}}_{\text {axion }}\right| \mathbf{L}, \mathbf{R}\right\rangle \\
= & \sum_{\mathbf{L}} \sum_{\mathbf{R}} \sum_{\mathbf{L}^{\prime}} \sum_{\mathbf{R}^{\prime}}\left\langle\mathbf{L}, \mathbf{R} \mid \Psi_{\text {axion }}\right\rangle \\
& \times\left\langle\Psi_{\text {axion }} \mid \mathbf{L}^{\prime}, \mathbf{R}^{\prime}\right\rangle\left\langle\mathbf{L}^{\prime}, \mathbf{R}^{\prime}\left|\widetilde{\mathcal{O}}_{\text {axion }}^{\mathbf{L}}\right| \mathbf{L}, \mathbf{R}\right\rangle \\
= & \sum_{\mathbf{L}} \sum_{\mathbf{R}} \sum_{\mathbf{L}^{\prime}} \sum_{\mathbf{R}^{\prime}}\left\langle\mathbf{L}, \mathbf{R} \mid \Psi_{\text {axion }}\right\rangle
\end{aligned}
$$




$$
\begin{aligned}
& \times\left\langle\Psi_{\text {axion }} \mid \mathbf{L}^{\prime}, \mathbf{R}^{\prime}\right\rangle\left\langle\mathbf{L}^{\prime}\left|\widetilde{\mathcal{O}}_{\text {axion }}^{\mathbf{L}}\right| \mathbf{L}\right\rangle \delta_{\mathbf{R R}^{\prime}} \\
= & \sum_{\mathbf{L}} \sum_{\mathbf{R}} \sum_{\mathbf{L}^{\prime}}\left\langle\mathbf{L}, \mathbf{R} \mid \Psi_{\text {axion }}\right\rangle \\
& \times\left\langle\Psi_{\text {axion }} \mid \mathbf{L}^{\prime}, \mathbf{R}^{\prime}\right\rangle\left\langle\mathbf{L}^{\prime}\left|\widetilde{\mathcal{O}}_{\text {axion }}^{\mathbf{L}}\right| \mathbf{L}\right\rangle \\
= & \operatorname{Tr}\left(\rho_{\text {axion }}^{\mathbf{L}} \widetilde{\mathcal{O}}_{\text {axion }}^{\mathbf{L}}\right),
\end{aligned}
$$

where the operator $\widetilde{\mathcal{O}}_{\text {axion }}^{\mathbf{L}}$ solely in the region $\mathbf{L}$ is defined by the following expression for NES formalism:

$$
\begin{aligned}
\left\langle\mathbf{L}^{\prime}, \mathbf{R}^{\prime}\left|\widetilde{\mathcal{O}}_{\text {axion }}^{\mathbf{L}}\right| \mathbf{L}, \mathbf{R}\right\rangle & =\left\langle\mathbf{L}^{\prime}\left|\widetilde{\mathcal{O}}_{\text {axion }}^{\mathbf{L}}\right| \mathbf{L}\right\rangle\left\langle\mathbf{R}^{\prime} \mid \mathbf{R}\right\rangle \\
& =\left\langle\mathbf{L}^{\prime}\left|\widetilde{\mathcal{O}}_{\text {axion }}^{\mathbf{L}}\right| \mathbf{L}\right\rangle \delta_{\mathbf{R R}^{\prime}}
\end{aligned}
$$

Also in NES formalism the density matrix $\rho_{\text {axion }}^{\mathbf{L}}$ for the region $\mathbf{L}$ is described by the following expression:

$$
\begin{aligned}
\rho_{\text {axion }}^{\mathbf{L}} & =\operatorname{Tr}_{\mathbf{R}} \rho_{\text {axion }} \\
& =\sum_{\mathbf{L}} \sum_{\mathbf{L}^{\prime}}|\mathbf{L}\rangle\left(\sum_{\mathbf{R}}\left\langle\mathbf{L}, \mathbf{R} \mid \Psi_{\text {axion }}\right\rangle\left\langle\Psi_{\text {axion }} \mid \mathbf{L}^{\prime}, \mathbf{R}^{\prime}\right\rangle\right)
\end{aligned}
$$

$\left\langle\mathbf{L}^{\prime}\right|$

$$
=\sum_{\mathbf{L}} \sum_{\mathbf{L}^{\prime}}|\mathbf{L}\rangle\left(\sum_{\mathbf{R}} \Psi_{\text {axion }}(\mathbf{L}, \mathbf{R}) \Psi_{\text {axion }}^{*}\left(\mathbf{L}^{\prime}, \mathbf{R}^{\prime}\right)\right)
$$

$\left\langle\mathbf{L}^{\prime}\right|$.

This implies that in NES formalism, the physica. is solely described by the information from the reg $\mathbf{J}$ and consequently the expectation value or ch operator satisfy the following condition:

$$
\begin{aligned}
\left\langle\widetilde{O}_{\text {axion }}\right\rangle & =\operatorname{Tr}\left(\rho_{\text {axion }} \widetilde{\mathcal{O}}_{\text {axion }}\right) \\
& =\operatorname{Tr}\left(\rho_{\text {axion }}^{\mathbf{L}} \widetilde{\mathcal{O}}_{\text {axion }}^{\mathbf{L}}\right)=
\end{aligned}
$$

The above analysis can help u 20 explain the differences between the power spe a of $n$ ean square vacuum fluctuation obtained fro $\quad \mathrm{F}^{\prime} \quad \mathrm{M}$ and NES formalism on large scale ( 0 small s. number or large wave length regime). It le. points towards the fact that in FOE and RDM fo malism. creation and annihilation operators for a ron field inciudes new set of creation and annihilation rato s coming from the Bogoliubov transfor11. on fru one quantum basis to the other. This means tha figld operator in the FOE formalism also involves . e extra creation and annihilation operators even if the computation is being performed on a particularly specified temporal slice defined in the region $\mathbf{L}$ of the Hilbert space. On the other hand, after applying the partial trace over the degrees of freedom from the region $\mathbf{R}$, the mixed quantum state, using which we formulate the RDM formalism, is prepared by the creation and annihilation operators in the region $\mathbf{L}$ of the Hilbert space. Thus, in RDM formalism, the field operator is only defined in the region $\mathbf{L}$ and not in the region $\mathbf{R}$ of the Hilbert space. This implies that the field operator defined before partially tracing over the degrees of freedom from region $\mathbf{R}$ for FOE formalism is different from the field operator in region $\mathbf{L}$ used in RDM formalism since for this case we have performed the partial trace over the egrees of freedom in region $\mathbf{R}$. Thus, any general qua 'm nechanical operator defined in the framework of FOE ot same as that of RDM formalism.

Before we conclude, we point ov hat apart from the quantification of the mean square vac am fluctuation in the formalisms we discuss there, wh have also computed the entanglement ent py o on Neumann measure and the Renyi entr py ur previous work [24,25].

Acknowledgements SC w / like to thank Quantum Gravity and Unified Theor, anc heoretical Cosmology Group, Max Planck Institute for Gra $\%$ on , Albert Einstein Institute for providing the Post-Doctoral $k$ arch Fellowship. SC take this opportunity to thank sincere $^{1 \times}$ to Jean- $\mathrm{L}$ Lehners, Shiraz Minwalla and Varun Sahni for their col no nort and inspiration. SC also thank the organisers of Advancel S ring School 2017, ST ${ }^{4} 2017$ and Kavli Asian Winter School on Strings, Particles and Cosmology 2018, Summer School on Cosmolgy 2018, 1CTP, Trieste, 15 th Marcel Grossman Meeting, Rome for viding the local hospitality during the work. SC also thank ICTP, Ti ste, La Sapienza University, Rome, DTP, TIFR, Mumbai, ICTS, $\mathrm{TJ} / \mathrm{R}$, Bengaluru, IOP, CMI, SINP and IACS for providing the academic visit during the work. SP acknowledges the J. C. Bose National Fellowship for support of his research. Last but not the least, we would like to acknowledge our debt to the people of India for their generous and steady support for research in natural sciences, especially for theoretical high energy physics, string theory and cosmology.

Data Availability Statement This manuscript has no associated data or the data will not be deposited. [Authors' comment: My paper not contain any data so that there is no point of depositing it. It is completely a theory paper.]

Open Access This article is licensed under a Creative Commons Attribution 4.0 International License, which permits use, sharing, adaptation, distribution and reproduction in any medium or format, as long as you give appropriate credit to the original author(s) and the source, provide a link to the Creative Commons licence, and indicate if changes were made. The images or other third party material in this article are included in the article's Creative Commons licence, unless indicated otherwise in a credit line to the material. If material is not included in the article's Creative Commons licence and your intended use is not permitted by statutory regulation or exceeds the permitted use, you will need to obtain permission directly from the copyright holder. To view a copy of this licence, visit http://creativecomm ons.org/licenses/by/4.0/.

Funded by $\mathrm{SCOAP}^{3}$.

\section{A Quantum correction to the power spectrum in FOE formalism}

At the superhorizon time scales $\left(t_{\mathbf{L}}>>1\right)$ of region $\mathbf{L}$ one can write the amplitude of the FOE power spectrum as: 


$$
\begin{aligned}
& \sum_{\sigma= \pm 1}\left|\widetilde{\chi^{\sigma}}\right|^{2}=\sum_{\sigma= \pm 1}\left(\widetilde{\chi^{\sigma}}\right)^{\dagger} \widetilde{\chi^{\sigma}} \\
& \stackrel{t_{\mathbf{L}}>1}{\longrightarrow} \widetilde{\mathcal{M ( p , v )}}\left(\cosh t_{\mathbf{L}}\right)^{2 v-1}
\end{aligned}
$$

where the time independent function $\widetilde{\mathcal{M}(p, v)}$ is defined as:

$$
\begin{aligned}
\widetilde{\mathcal{M}(p, v)}= & \frac{2^{2 v-1}(\Gamma(v))^{2}}{\pi} \times \sum_{\sigma= \pm 1}\left[\frac{\left(\left|\mathcal{A}_{\mathbf{L}}^{\sigma}\right|^{2}+\left|\mathcal{B}_{\mathbf{L}}^{\sigma}\right|^{2}\right)}{\left|\Gamma\left(v+i p+\frac{1}{2}\right)\right|^{2}}\right. \\
& +\frac{\mathcal{A}_{\mathbf{L}}^{\sigma} \mathcal{B}_{\mathbf{L}}^{\sigma *}}{\left(\Gamma\left(v-i p+\frac{1}{2}\right)\right)^{2}}+\frac{\mathcal{A}_{\mathbf{L}}^{\sigma *} \mathcal{B}_{\mathbf{L}}^{\sigma}}{\left(\Gamma\left(v+i p+\frac{1}{2}\right)\right)^{2}} \\
& +\sum_{n=0}^{\infty}\left\{\frac{\left(\mathcal{A}_{\mathbf{L}}^{\sigma} \mathcal{A}_{\mathbf{L},(n)}^{\sigma *}+\mathcal{B}_{\mathbf{L}}^{\sigma} \mathcal{B}_{\mathbf{L},(n)}^{\sigma *}\right)}{\Gamma\left(v-i p+\frac{1}{2}\right) \Gamma\left(v+i p_{n}+\frac{1}{2}\right)}\right. \\
& +\frac{\left(\mathcal{A}_{\mathbf{L}}^{\sigma} \mathcal{B}_{\mathbf{L},(n)}^{\sigma *}+\mathcal{A}_{\mathbf{L},(n)}^{\sigma} \mathcal{B}_{\mathbf{L}}^{\sigma *}\right)}{\Gamma\left(v-i p+\frac{1}{2}\right) \Gamma\left(v-i p_{n}+\frac{1}{2}\right)} \\
& \left.+\frac{\left(\mathcal{A}_{\mathbf{L},(n)}^{\sigma *} \mathcal{B}_{\mathbf{L}}^{\sigma}+\mathcal{A}_{\mathbf{L}}^{\sigma *} \mathcal{B}_{\mathbf{L},(n)}^{\sigma}\right)}{\Gamma\left(v+i p_{n}+\frac{1}{2}\right) \Gamma\left(v+i p+\frac{1}{2}\right)}\right\} \\
& +\sum_{n=0}^{\infty} \sum_{m=0}^{\infty}\left\{\frac{\left(\mathcal{A}_{\mathbf{L},(n)}^{\sigma} \mathcal{A}_{\mathbf{L},(m)}^{\sigma *}+\mathcal{B}_{\mathbf{L},(n)}^{\sigma} \mathcal{B}_{\mathbf{L},(m)}^{\sigma *}\right)}{\left.\mathcal{A}_{(v, i n)}^{\sigma}-i p_{n}+\frac{1}{2}\right) \Gamma\left(v+i p_{m}+\frac{1}{2}\right)}\right. \\
& +\frac{\mathcal{B}_{\mathbf{L},(m)}^{\sigma *}}{\Gamma\left(v-i p_{n}+\frac{1}{2}\right) \Gamma\left(v-i p_{m}+\frac{1}{2}\right)} \\
& +\frac{\mathcal{A}_{\mathbf{L},(n)}^{\sigma *} \mathcal{B}_{\mathbf{L},(m)}^{\sigma}}{\Gamma\left(v+i p_{n}+\frac{1}{2}\right) \Gamma\left(v+i p_{m}+\frac{1}{2}\right)}
\end{aligned}
$$

A.1 For large wave number

Further to know the exact wave number mpendence of the amplitude of the normalized pown from Bunch Davies vacuum we need to ${ }^{1}$ now the behaviour of the power spectrum at very short vave ngths $\left., p, p_{n}>>1\right)$. After taking this limit it is eynec. That une power spectrum of axion match with the $r$ ult obtan $d$ for spatially flat universe. In general for an rbit. value of the mass parameter $v$, we get the followi approxit ated contributions in the short wavelength 1 it $\left(\eta, p_{\lambda}>>1\right)$, which are explicitly appearing in the expr ion $f$ or the amplitude of the normalized power spectr i from sunch Davies vacuum:

$\sum_{\sigma= \pm 1} \frac{\left|\mathcal{A}_{\mathbf{L}}^{\sigma}\right|^{2}}{\left|\Gamma\left(v+i p+\frac{1}{2}\right)\right|^{2}} \stackrel{p>>1}{\approx} \frac{\pi e^{-\pi p}}{2 p^{4}|\Gamma(i p)|^{2}}$,

$\sum_{\sigma= \pm 1} \frac{\left|\mathcal{B}_{\mathbf{L}}^{\sigma}\right|^{2}}{\left|\Gamma\left(v+i p+\frac{1}{2}\right)\right|^{2}} \stackrel{p>>1}{\approx} \frac{\pi e^{-5 \pi p}}{2 p^{4}|\Gamma(i p)|^{2}}$,

$$
\sum_{\sigma= \pm 1} \frac{\mathcal{A}_{\mathbf{L}}^{\sigma} \mathcal{B}_{\mathbf{L}}^{\sigma *}}{\left(\Gamma\left(v-i p+\frac{1}{2}\right)\right)^{2}} \stackrel{p \gg>1}{\approx} \frac{\pi e^{-3 \pi p}}{2 p^{4}|\Gamma(i p)|^{2}},
$$

$$
\sum_{\sigma= \pm 1} \frac{\mathcal{A}_{\mathbf{L}}^{\sigma *} \mathcal{B}_{\mathbf{L}}^{\sigma}}{\left(\Gamma\left(v+i p+\frac{1}{2}\right)\right)^{2}} \stackrel{p \gg 1}{\approx} \frac{\pi e^{-3 \pi p}}{2 p^{4}|\Gamma(i p)|^{2}}
$$

$$
\sum_{\sigma= \pm 1} \frac{\mathcal{A}_{\mathbf{L}}^{\sigma} \mathcal{A}_{\mathbf{L},(n)}^{\sigma *}}{\Gamma\left(v-i p+\frac{1}{2}\right) \Gamma\left(v+i p_{n}+\frac{1}{2}\right)}
$$

$$
\begin{gathered}
p, p_{n>>1} \frac{\pi e^{-\pi\left(p+p_{n}\right) / 2}}{2 p^{2} p_{n}^{2}|\Gamma(i p)|\left|\Gamma\left(i p_{n}\right)\right|} \\
\sum_{\sigma= \pm 1} \frac{\mathcal{B}_{\mathbf{L}}^{\sigma} \mathcal{B}_{\mathbf{L},(n)}^{\sigma *}}{\Gamma\left(v-i p+\frac{1}{2}\right) \Gamma\left(v+i p_{n}+{ }^{\prime}\right)}
\end{gathered}
$$$$
\stackrel{p, p_{n}>>1}{\approx} \frac{\pi e^{-5 \pi\left(p+p_{n}\right) / 2}}{\left.2 p^{2} p_{n}^{2}|\Gamma(i p)||\Gamma| i p_{n}\right) \mid}
$$
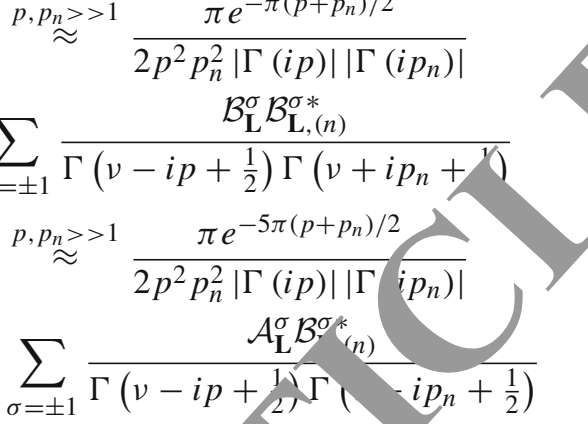

$\stackrel{p, p_{n}>>}{\approx}$

$$
\left.\pi e^{-}+5 p_{n}\right) / 2
$$

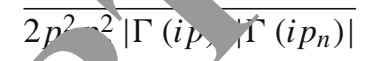

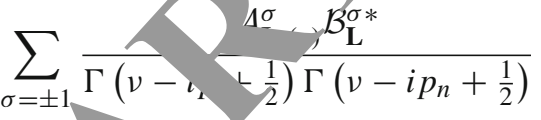

$$
\stackrel{p, p_{n}}{\approx} \sqrt{2 p^{2} p_{n}^{2}|\Gamma(i p)|\left|\Gamma\left(i p_{n}\right)\right|}
$$

$$
\bigcup_{ \pm 1} \overline{\Gamma\left(v+i p+\frac{1}{2}\right) \Gamma\left(v+i p_{n}+\frac{1}{2}\right)}
$$$$
\stackrel{p, p_{n}>>1}{\approx} \frac{\pi e^{-\pi\left(5 p+p_{n}\right) / 2}}{2 p^{2} p_{n}^{2}|\Gamma(i p)|\left|\Gamma\left(i p_{n}\right)\right|}
$$

$$
\sum_{\sigma= \pm 1} \frac{\mathcal{A}_{\mathbf{L}}^{\sigma *} \mathcal{B}_{\mathbf{L},(n)}^{\sigma}}{\Gamma\left(v+i p+\frac{1}{2}\right) \Gamma\left(v+i p_{n}+\frac{1}{2}\right)}
$$

$$
p, \stackrel{p, p_{n}>>1}{\approx} \frac{\pi e^{-\pi\left(p+5 p_{n}\right) / 2}}{2 p^{2} p_{n}^{2}|\Gamma(i p)|\left|\Gamma\left(i p_{n}\right)\right|}
$$

$$
\begin{gathered}
\sum_{\sigma= \pm 1} \frac{\mathcal{A}_{\mathbf{L},(n)}^{\sigma} \mathcal{A}_{\mathbf{L},(m)}^{\sigma *}}{\Gamma\left(v-i p_{n}+\frac{1}{2}\right) \Gamma\left(v+i p_{m}+\frac{1}{2}\right)} \\
\stackrel{p_{n}, p_{m}>>1}{\approx} \frac{\pi e^{-\pi\left(p_{n}+p_{m}\right) / 2}}{2 p_{n}^{2} p_{m}^{2}\left|\Gamma\left(i p_{m}\right)\right|\left|\Gamma\left(i p_{n}\right)\right|}
\end{gathered}
$$$$
\sum_{\sigma= \pm 1} \frac{\mathcal{B}_{\mathbf{L},(n)}^{\sigma} \mathcal{B}_{\mathbf{L},(m)}^{\sigma *}}{\Gamma\left(v-i p_{n}+\frac{1}{2}\right) \Gamma\left(v+i p_{m}+\frac{1}{2}\right)}
$$

$$
\begin{aligned}
& \stackrel{p_{n}, p_{m}>>1}{\approx} \frac{\pi e^{-5 \pi\left(p_{n}+p_{m}\right) / 2}}{2 p_{n}^{2} p_{m}^{2}\left|\Gamma\left(i p_{m}\right)\right|\left|\Gamma\left(i p_{n}\right)\right|} \\
& \sum_{\sigma= \pm 1} \frac{\mathcal{A}_{\mathbf{L},(n)}^{\sigma} \mathcal{B}_{\mathbf{L},(m)}^{\sigma *}}{\Gamma\left(v-i p_{n}+\frac{1}{2}\right) \Gamma\left(v-i p_{m}+\frac{1}{2}\right)}
\end{aligned}
$$$$
\stackrel{p_{n}, p_{m}>>1}{\approx} \frac{\pi e^{-3 \pi\left(p_{n}+p_{m}\right) / 2}}{2 p_{n}^{2} p_{m}^{2}\left|\Gamma\left(i p_{m}\right)\right|\left|\Gamma\left(i p_{n}\right)\right|}
$$$$
\sum_{\sigma= \pm 1} \frac{\mathcal{A}_{\mathbf{L},(n)}^{\sigma *} \mathcal{B}_{\mathbf{L},(m)}^{\sigma}}{\Gamma\left(v+i p_{n}+\frac{1}{2}\right) \Gamma\left(v+i p_{m}+\frac{1}{2}\right)}
$$ 


$$
p_{n}, \underset{m}{\approx}>>1 \frac{\pi e^{-3 \pi\left(p_{n}+p_{m}\right) / 2}}{2 p_{n}^{2} p_{m}^{2}\left|\Gamma\left(i p_{m}\right)\right|\left|\Gamma\left(i p_{n}\right)\right|}
$$

Further, we apply Stirling's formula to approximate Gamma functions for large wavenumbers $p, p_{n}>>1$ to simplify the expression for the power spectrum:

$$
\begin{aligned}
& \Gamma(i p) \sim \sqrt{2 \pi}(i p)^{i p-\frac{1}{2}} e^{-i p}\left(1+\frac{1}{12 i p}-\frac{1}{288 p^{2}}+\cdots\right), \\
& \Gamma\left(i p_{n}\right) \sim \sqrt{2 \pi}\left(i p_{n}\right)^{i p_{n}-\frac{1}{2}} e^{-i p_{n}} \\
&\left(1+\frac{1}{12 i p_{n}}-\frac{1}{288 p_{n}^{2}}+\cdots\right) .
\end{aligned}
$$

Consequently, we get the following simplified expressions in large wavenumber $\left(p, p_{n}>>1\right)$ limit:

$$
\begin{aligned}
& \sum_{\sigma= \pm 1} \frac{\left|\mathcal{A}_{\mathbf{L}}^{\sigma}\right|^{2}}{\left|\Gamma\left(v+i p+\frac{1}{2}\right)\right|^{2}} \sim \frac{1}{2 p^{3}\left(1+\frac{1}{82944 p^{4}}\right)} \\
& \sum_{\sigma= \pm 1} \frac{\left|\mathcal{B}_{\mathbf{L}}^{\sigma}\right|^{2}}{\left|\Gamma\left(v+i p+\frac{1}{2}\right)\right|^{2}} \sim \frac{e^{-4 \pi p}}{2 p^{3}\left(1+\frac{1}{82944 p^{4}}\right)} \\
& \sum_{\sigma= \pm 1} \frac{\mathcal{A}_{\mathbf{L}}^{\sigma} \mathcal{B}_{\mathbf{L}}^{\sigma *}}{\left(\Gamma\left(v-i p+\frac{1}{2}\right)\right)^{2}} \sim \frac{e^{-2 \pi p}}{2 p^{3}\left(1+\frac{1}{82944 p^{4}}\right)}, \\
& \sum_{\sigma= \pm 1} \frac{\mathcal{A}_{\mathbf{L}}^{\sigma *} \mathcal{B}_{\mathbf{L}}^{\sigma}}{\left(\Gamma\left(v+i p+\frac{1}{2}\right)\right)^{2}} \sim \frac{e^{-2 \pi p}}{2 p^{3}\left(1+\frac{1}{8294 \cdot p^{4}}\right)} \\
& \sum_{\sigma= \pm 1} \frac{\mathcal{A}_{\mathbf{L}}^{\sigma} \mathcal{A}_{\mathbf{L},(n)}^{\sigma *}}{\Gamma\left(v-i p+\frac{1}{2}\right) \Gamma\left(v+i p_{n}+\frac{1}{2}\right.} \\
& \sim \frac{1}{2 p^{3 / 2} p_{n}^{3 / 2} \sqrt{1+\frac{1}{82944 p^{4}}} \sqrt{1+\sqrt{82944 p_{n}^{4}}}}
\end{aligned}
$$

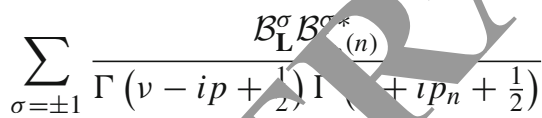

$$
\begin{aligned}
& \sim \frac{-2 \pi\left(p+\eta_{n}\right)}{2 p^{3 / 2} p_{n}^{3 / 2} \sqrt{1+\frac{1}{\sigma 2944 p^{4}}} \sqrt{1+\frac{1}{82944 p_{n}^{4}}}} \\
& \sum_{\sigma= \pm 1} \frac{{ }_{\mathbf{L}}^{\sigma} \mathcal{B}_{\mathbf{L},(n)}^{\sigma *}}{\left(v-y+\frac{1}{2}\right) \Gamma\left(v-i p_{n}+\frac{1}{2}\right)} \\
& 2 p^{3 / 2} p_{n}^{3 / 2} \sqrt{1+\frac{1}{82944 p^{4}}} \sqrt{1+\frac{1}{82944 p_{n}^{4}}} \\
& \sum_{\sigma= \pm 1} \frac{\mathcal{A}_{\mathbf{L},(n)}^{\sigma} \mathcal{B}_{\mathbf{L}}^{\sigma *}}{\Gamma\left(v-i p+\frac{1}{2}\right) \Gamma\left(v-i p_{n}+\frac{1}{2}\right)} \\
& \sim \frac{e^{-2 \pi p}}{2 p^{3 / 2} p_{n}^{3 / 2} \sqrt{1+\frac{1}{82944 p^{4}}} \sqrt{1+\frac{1}{82944 p_{n}^{4}}}}
\end{aligned}
$$

$$
\begin{gathered}
\sum_{\sigma= \pm 1} \frac{\mathcal{A}_{\mathbf{L},(n)}^{\sigma *} \mathcal{B}_{\mathbf{L}}^{\sigma}}{\Gamma\left(v+i p+\frac{1}{2}\right) \Gamma\left(v+i p_{n}+\frac{1}{2}\right)} \\
\sim \frac{e^{-2 \pi p}}{2 p^{3 / 2} p_{n}^{3 / 2} \sqrt{1+\frac{1}{82944 p^{4}}} \sqrt{1+\frac{1}{82944 p_{n}^{4}}}}
\end{gathered}
$$

$$
\begin{gathered}
\sum_{\sigma= \pm 1} \frac{\mathcal{A}_{\mathbf{L}}^{\sigma *} \mathcal{B}_{\mathbf{L},(n)}^{\sigma}}{\Gamma\left(v+i p+\frac{1}{2}\right) \Gamma\left(v+i p_{n}+\frac{1}{2}\right)} \\
\sim \frac{e^{-2 \pi p_{n}}}{2 p^{3 / 2} p_{n}^{3 / 2} \sqrt{1+\frac{1}{82944 p^{4}}} \sqrt{1 千+} \frac{1}{82}}
\end{gathered}
$$

$$
\begin{aligned}
& \sum_{\sigma= \pm 1} \frac{\mathcal{A}_{\mathbf{L},(n)}^{\sigma} \mathcal{A}_{\mathbf{L},(m)}^{\sigma *}}{\left.\Gamma\left(v-i p_{n}+\frac{1}{2}\right) \Gamma / v+\eta_{\eta}+\frac{1}{2}\right)} \\
& \sim \frac{1}{2 p_{m}^{3 / 2} p_{n}^{3 / 2} \sqrt{\pi}} \frac{1}{\frac{1}{944 p_{m}^{4}}} \sqrt{1+\frac{1}{82944 p_{n}^{4}}}
\end{aligned}
$$

$$
\sum_{\sigma= \pm 1} \sum_{i p} \frac{\mathcal{B}_{\mathbf{L},(n)}^{\sigma} \mathcal{D}_{\mathbf{L},(m)}}{\Gamma\left(v+i p_{m}+\frac{1}{2}\right)}
$$

$$
\sum_{2,1 m} \frac{\mathcal{L}^{-2 \pi\left(p_{n}+p_{m}\right)}}{\Gamma\left(v-i p_{n}+\frac{1}{2}\right) \Gamma\left(v-i p_{m}+\frac{1}{2}\right)}
$$

$$
\begin{gathered}
\sim \frac{e^{-\pi\left(p_{n}+p_{m}\right)}}{2 p_{m}^{3 / 2} p_{n}^{3 / 2} \sqrt{1+\frac{1}{82944 p_{m}^{4}}} \sqrt{1+\frac{1}{82944 p_{n}^{4}}}} \\
\sum_{\sigma= \pm 1} \frac{\mathcal{A}_{\mathbf{L},(n)}^{\sigma *} \mathcal{B}_{\mathbf{L},(m)}^{\sigma}}{\Gamma\left(v+i p_{n}+\frac{1}{2}\right) \Gamma\left(v+i p_{m}+\frac{1}{2}\right)} \\
\sim \frac{e^{-\pi\left(p_{n}+p_{m}\right)}}{2 p_{m}^{3 / 2} p_{n}^{3 / 2} \sqrt{1+\frac{1}{82944 p_{m}^{4}}} \sqrt{1+\frac{1}{82944 p_{n}^{4}}}}
\end{gathered}
$$

As a result, in the short wave length approximation the time independent function $\mathcal{M}(\widetilde{p>>} 1, v)$ for any arbitrary mass parameter $v$ can be expressed as:

$\mathcal{M}(\widetilde{p>>} 1, v)=\frac{2^{2(\nu-1)}(\Gamma(\nu))^{2}}{p^{3} \pi} \mathcal{G}(\widetilde{p>>} 1)$,

where we define a new function $\mathcal{G}(\widetilde{p>>}$ ) in the short wave length limit as given by:

$$
\begin{aligned}
\widetilde{\mathcal{G}(p)}= & \frac{1}{\left(1+\frac{1}{82944 p^{4}}\right)}\left[\left(1+e^{-2 \pi p}\right)^{2}+\sum_{n=0}^{\infty}\left(\frac{p}{p_{n}}\right)^{\frac{3}{2}}\right. \\
& \times \frac{\sqrt{1+\frac{1}{82944 p^{4}}}}{\sqrt{1+\frac{1}{82944 p_{n}^{4}}}}\left(1+2\left(e^{-2 \pi p}+e^{-2 \pi p_{n}}\right)+e^{-2 \pi\left(p+p_{n}\right)}\right) \\
& +\sum_{n=0}^{\infty} \sum_{m=0}^{\infty} \frac{p^{3}}{\left(p_{n} p_{m}\right)^{3 / 2}}
\end{aligned}
$$




$$
\left.\times \frac{\left(1+\frac{1}{82944 p^{4}}\right)}{\sqrt{1+\frac{1}{82944 p_{n}^{4}}} \sqrt{1+\frac{1}{82944 p_{m}^{4}}}}\left(1+e^{-\pi\left(p_{m}+p_{n}\right)}\right)^{2}\right],
$$

\section{A.2 For small wave number}

Similarly to know the exact wavenumber dependence of the amplitude of the normalised power spectrum from Bunch Davies vacuum in the long wavelength limit we need to know the behaviour of the power spectrum for $p, p_{n}<<1$. In this limit it is expected that the power spectrum of axion should match with the result obtained for spatially flat universe. In general for an arbitrary value of the mass parameter $v$, we get the following approximated contributions in the long wavelength limit $\left(p, p_{n}<<1\right)$, which are explicitly appearing in the expression for the amplitude of the normalised power spectrum from Bunch Davies vacuum:

$$
\begin{aligned}
& \sum_{\sigma= \pm 1} \frac{\left|\mathcal{A}_{\mathbf{L}}^{\sigma}\right|^{2}}{\left|\Gamma\left(v+i p+\frac{1}{2}\right)\right|^{2}} \stackrel{p<1}{\approx} \frac{\pi}{4\left|\Gamma\left(v+\frac{1}{2}\right)\right|^{2}}, \\
& \sum_{\sigma= \pm 1} \frac{\left|\mathcal{B}_{\mathbf{L}}^{\sigma}\right|^{2}}{\left|\Gamma\left(v+i p+\frac{1}{2}\right)\right|^{2}} \stackrel{p<1}{\approx} \frac{\pi}{4\left|\Gamma\left(v+\frac{1}{2}\right)\right|^{2}}, \\
& \sum_{\sigma= \pm 1} \frac{\mathcal{A}_{\mathbf{L}}^{\sigma} \mathcal{B}_{\mathbf{L}}^{\sigma *}}{\left(\Gamma\left(v-i p+\frac{1}{2}\right)\right)^{2}} \stackrel{p<<1}{\approx} \frac{\pi}{4\left(\Gamma\left(v+\frac{1}{2}\right)\right)^{2}}, \\
& \sum_{\sigma= \pm 1} \frac{\mathcal{A}_{\mathbf{L}}^{\sigma *} \mathcal{B}_{\mathbf{L}}^{\sigma}}{\left(\Gamma\left(v+i p+\frac{1}{2}\right)\right)^{2}} \stackrel{p<<1}{\approx} \frac{\pi}{4\left(\Gamma\left(y+\frac{1}{2}\right)\right)^{2}} \\
& \sum_{\sigma= \pm 1} \frac{\mathcal{A}_{\mathbf{L}}^{\sigma} \mathcal{A}_{\mathbf{L},(n)}^{\sigma *}}{\Gamma\left(v-i p+\frac{1}{2}\right) \Gamma\left(v+i p_{n}+\frac{1}{2}\right)} \\
& \stackrel{p, p_{n}<<1}{\approx} \frac{\pi e^{-\pi\left(p+p_{n}\right)}}{2\left(\Gamma\left(v+\frac{1}{2}\right)\right.} \\
& \sum_{\sigma= \pm 1} \frac{\mathcal{B}_{\tau}^{\sigma} \mathcal{D}_{\mathbf{L}}}{\Gamma(v-i p} \frac{1}{\left.\frac{1}{2}\right) \Gamma(v} \frac{\left.1 p_{n}+\frac{1}{2}\right)}{\Gamma(1)} \\
& p, p_{n}<<1 \frac{\left.\pi e^{-\pi(}\right)}{\left.2 \vee \Gamma\left(x+\frac{1}{2}\right)\right)^{2}} \\
& \sum_{1} \frac{\mathcal{A}_{\mathbf{L}}^{\sigma} \mathcal{B}_{\mathbf{L},(n)}^{\sigma *}}{\left(1, i p+\frac{1}{2}\right) \Gamma\left(v-i p_{n}+\frac{1}{2}\right)} \\
& p_{p} \approx \frac{\pi e^{-\pi\left(p+p_{n}\right)}}{2\left(\Gamma\left(v+\frac{1}{2}\right)\right)^{2}} \\
& \sum_{\sigma= \pm 1} \frac{\mathcal{A}_{\mathbf{L},(n)}^{\sigma} \mathcal{B}_{\mathbf{L}}^{\sigma *}}{\Gamma\left(v-i p+\frac{1}{2}\right) \Gamma\left(v-i p_{n}+\frac{1}{2}\right)} \\
& \stackrel{p, p_{n}<<1}{\approx} \frac{\pi e^{-\pi\left(p+p_{n}\right)}}{2\left(\Gamma\left(v+\frac{1}{2}\right)\right)^{2}}
\end{aligned}
$$

$$
\begin{gathered}
\sum_{\sigma= \pm 1} \frac{\mathcal{A}_{\mathbf{L},(n)}^{\sigma *} \mathcal{B}_{\mathbf{L}}^{\sigma}}{\Gamma\left(v+i p+\frac{1}{2}\right) \Gamma\left(v+i p_{n}+\frac{1}{2}\right)} \\
\stackrel{p, p_{n}<<1}{\approx} \frac{\pi e^{-\pi\left(p+p_{n}\right)}}{2\left(\Gamma\left(v+\frac{1}{2}\right)\right)^{2}}
\end{gathered}
$$

$$
\sum_{\sigma= \pm 1} \frac{\mathcal{A}_{\mathbf{L}}^{\sigma *} \mathcal{B}_{\mathbf{L},(n)}^{\sigma}}{\Gamma\left(v+i p+\frac{1}{2}\right) \Gamma\left(v+i p_{n}+\frac{1}{2}\right)}
$$$$
\stackrel{p, p_{n}<<1}{\approx} \frac{\pi e^{-\pi\left(p+p_{n}\right)}}{2\left(\Gamma\left(v+\frac{1}{2}\right)\right)^{2}}
$$$$
\sum_{\sigma= \pm 1} \frac{\mathcal{A}_{\mathbf{L},(n)}^{\sigma} \mathcal{A}_{\mathbf{L},(m)}^{\sigma *}}{\Gamma\left(v-i p_{n}+\frac{1}{2}\right) \Gamma\left(v-i p_{m}+\frac{1}{2}\right)}
$$

$$
p_{n}, p_{m}<<1 \frac{\pi e^{-\pi\left(p_{n}+\pi\right.}}{2\left(\Gamma\left(1-\frac{1}{2}\right)\right)^{2}}
$$$$
\sum_{\sigma= \pm 1} \frac{\beta_{\mathbf{L},(n)}^{\sigma}{ }^{\sigma *}(v)}{\Gamma\left(v-i p_{i}+\frac{1}{2}\right) \Gamma\left(v+i p_{m}+\frac{1}{2}\right)}
$$

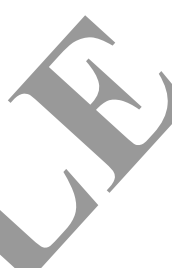

$$
\stackrel{p_{n}, p_{m}<<1}{\approx} \frac{-\pi\left(p_{n}+p_{m}\right)}{2\left(\Gamma\left(v+\frac{1}{2}\right)\right)^{2}}
$$$$
\sum_{\tau= \pm 1} \frac{\mathcal{A}_{\mathbf{L},(n)}^{\sigma} \mathcal{B}_{\mathbf{L},(m)}^{\sigma *}}{\Gamma\left(\mathcal{L}-i p_{n}+\frac{1}{2}\right) \Gamma\left(v-i p_{m}+\frac{1}{2}\right)}
$$

$$
\stackrel{n, p_{m}}{\approx}<<\frac{\pi e^{-\pi\left(p_{n}+p_{m}\right)}}{2\left(\Gamma\left(v+\frac{1}{2}\right)\right)^{2}}
$$$$
\sum_{\sigma= \pm 1} \frac{\mathcal{A}_{\mathbf{L},(n)}^{\sigma *} \mathcal{B}_{\mathbf{L},(m)}^{\sigma}}{\Gamma\left(v+i p_{n}+\frac{1}{2}\right) \Gamma\left(v+i p_{m}+\frac{1}{2}\right)}
$$

$$
p_{n}, \stackrel{p_{m}}{\approx}<<1 \frac{\pi e^{-\pi\left(p_{n}+p_{m}\right)}}{2\left(\Gamma\left(v+\frac{1}{2}\right)\right)^{2}} .
$$

As a result, the time independent function $\mathcal{M}(\widetilde{p<<}, v)$ for any arbitrary mass parameter $v$ can be expressed as:

$\mathcal{M}(\widetilde{p<<1}, v)=\frac{2^{2(\nu-1)}(\Gamma(v))^{2}}{\pi} \mathcal{G}(\widetilde{p<<1})$,

where we define a new function $\mathcal{G}(\widetilde{p<<})$ in the long wave length limit as given by:

$$
\begin{gathered}
\mathcal{G}(\widetilde{p<<})=\frac{\pi}{\left|\Gamma\left(v+\frac{1}{2}\right)\right|^{2}}\left[1+\frac{\left|\Gamma\left(v+\frac{1}{2}\right)\right|^{2}}{\left(\Gamma\left(v+\frac{1}{2}\right)\right)^{2}}\right. \\
\left.\left\{1+3 e^{-\pi p} \sum_{n=0}^{\infty} e^{-\pi p_{n}}+2 \sum_{n=0}^{\infty} \sum_{m=0}^{\infty} e^{-\pi\left(p_{n}+p_{m}\right)}\right\}\right] .
\end{gathered}
$$




\section{B Quantum correction to the power spectrum in RDM formalism}

At the super horizon time scales $\left(t_{\mathbf{L}}>>1\right)$ of region $\mathbf{L}$ one can write the amplitude of the RDM power spectrum as:

$$
\begin{aligned}
& \left|\tilde{\psi}_{\mathbf{T}}^{\mathbf{L}}\right|^{2}=\left(\tilde{\psi}_{\mathbf{T}}^{\mathbf{L}}\right)^{\dagger} \tilde{\psi}_{\mathbf{T}}^{\mathbf{L}} \\
& \stackrel{t_{\mathbf{L}}>>1}{\longrightarrow} \widetilde{\mathcal{Q} p, \alpha, v)}\left(\cosh t_{\mathbf{L}}\right)^{2 v-1}
\end{aligned}
$$

where the time independent function $\widetilde{\mathcal{Q}(p, \alpha, v)}$ for generalised $\alpha$ vacua is defined as:

$$
\begin{aligned}
& \widetilde{\mathcal{Q}(p, \alpha, v)}=\frac{2^{2 v-1}(\Gamma(v))^{2}}{\pi} \\
& \times\left[\frac{\left(\left|\mathcal{E}_{\mathbf{L}}\right|^{2}+\left|\mathcal{F}_{\mathbf{L}}\right|^{2}\right)}{\left|\Gamma\left(v+i p+\frac{1}{2}\right)\right|^{2}}\right. \\
& +\frac{\mathcal{E}_{\mathbf{L}} \mathcal{F}_{\mathbf{L}}^{*}}{\left(\Gamma\left(v-i p+\frac{1}{2}\right)\right)^{2}}+\frac{\mathcal{E}_{\mathbf{L}}^{*} \mathcal{F}_{\mathbf{L}}}{\left(\Gamma\left(v+i p+\frac{1}{2}\right)\right)^{2}} \\
& +\sum_{n=0}^{\infty}\left\{\frac{\left(\mathcal{E}_{\mathbf{L}} \mathcal{E}_{\mathbf{L},(n)}^{*}+\mathcal{F}_{\mathbf{L}} \mathcal{F}_{\mathbf{L},(n)}^{*}\right)}{\Gamma\left(v-i p+\frac{1}{2}\right) \Gamma\left(v+i p_{n}+\frac{1}{2}\right)}\right. \\
& +\frac{\left(\mathcal{E}_{\mathbf{L}} \mathcal{F}_{\mathbf{L},(n)}^{*}+\mathcal{E}_{\mathbf{L},(n)} \mathcal{F}_{\mathbf{L}}^{*}\right)}{\Gamma\left(v-i p+\frac{1}{2}\right) \Gamma\left(v-i p_{n}+\frac{1}{2}\right)} \\
& +\frac{\left(\mathcal{E}_{\mathbf{L},(n)}^{*} \mathcal{F}_{\mathbf{L}}+\mathcal{E}_{\mathbf{L}}^{*} \mathcal{F}_{\mathbf{L},(n)}\right)}{\Gamma\left(v+i p+\frac{1}{2}\right) \Gamma\left(v+i p_{n}\right.}
\end{aligned}
$$

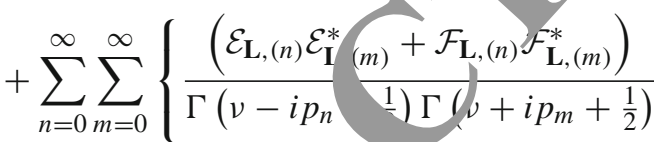

$$
\begin{aligned}
& +\frac{\mathcal{E}_{\mathbf{L},(n)}}{\Gamma\left(v-i p+\frac{1}{2}\right) \Gamma\left(v-i p_{m}+\frac{1}{2}\right)}
\end{aligned}
$$

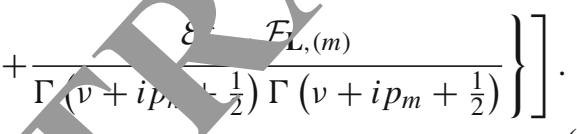

\section{B.1 For 1 rge wave number}

Fur to kn the exact wave number dependence of the 2. lit,$\quad f$ the normalised power spectrum from generalis vacua we need to know the behaviour of the power spectru,$\Lambda$ at very short wavelengths $\left(p, p_{n}>>1\right)$. After taking this limit it is expected that the power spectrum of axion should match with the result obtained for spatially flat universe. In general for an arbitrary value of the mass parameter $v$, we get the following approximated contributions in the short wavelength limit $\left(p, p_{n}>>1\right)$, which are explicitly appearing in the expression for the amplitude of the nor- malised power spectrum from generalised $\alpha$ vacua:

$\frac{\left|\mathcal{E}_{\mathbf{L}}\right|^{2}}{\left|\Gamma\left(v+i p+\frac{1}{2}\right)\right|^{2}} \stackrel{p>>1}{\approx} \frac{\pi e^{-\pi p}}{2 p^{4}|\Gamma(i p)|^{2}}$,

$\frac{\left|\mathcal{F}_{\mathbf{L}}\right|^{2}}{\left|\Gamma\left(v+i p+\frac{1}{2}\right)\right|^{2}} \stackrel{p>>1}{\approx} \frac{\pi e^{-5 \pi p}}{2 p^{4}|\Gamma(i p)|^{2}}$,

$\frac{\mathcal{E}_{\mathbf{L}} \mathcal{F}_{\mathbf{L}}^{*}}{\left(\Gamma\left(v-i p+\frac{1}{2}\right)\right)^{2}} \stackrel{p>1}{\approx} \frac{\pi e^{-3 \pi p}}{\left.2 p^{4} \mid \Gamma \text { i } p p\right) \mid}$

$$
\frac{\mathcal{E}_{\mathbf{L}}^{*} \mathcal{F}_{\mathbf{L}}}{\left(\Gamma\left(v+i p+\frac{1}{2}\right)\right)^{2}} \stackrel{p>>1}{\approx} \frac{J}{2 p^{4}} \frac{e^{-3 \pi p}}{(i p))^{1}}
$$

$\frac{\mathcal{E}_{\mathbf{L}} \mathcal{E}_{\mathbf{L},(n)}^{*}}{\Gamma\left(v-i p+\frac{1}{2}\right) \Gamma\left(v-i p_{n}+\frac{1}{2}\right)}$

$$
\stackrel{p, p_{n}>>1}{\approx} \overbrace{v^{2} p}^{\frac{\left.e^{-\pi(p}\right)^{/ / 2}}{|\Gamma(i p)|\left|\Gamma\left(i p_{n}\right)\right|}}
$$$$
\mathcal{F}_{\mathbf{L}}{ }^{*}(n)
$$

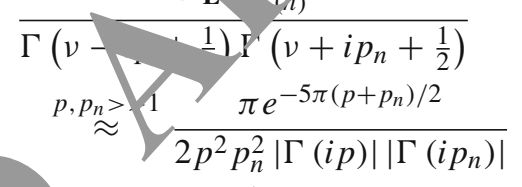

$$
\frac{\mathcal{E}_{\mathbf{L}} \mathcal{F}_{\mathbf{L},(n)}^{*}}{\Gamma\left(v-i p+\frac{1}{2}\right) \Gamma\left(v-i p_{n}+\frac{1}{2}\right)}
$$

$$
p, p_{n}>>1 \frac{\pi e^{-\pi\left(p+5 p_{n}\right) / 2}}{2 p^{2} p_{n}^{2}|\Gamma(i p)|\left|\Gamma\left(i p_{n}\right)\right|}
$$$$
\mathcal{E}_{\mathbf{L},(n)} \mathcal{F}_{\mathbf{L}}^{*}
$$

$$
\begin{gathered}
\frac{\mathcal{E}_{\mathbf{L},(n)} \mathcal{F}_{\mathbf{L}}^{*}}{\Gamma\left(v-i p+\frac{1}{2}\right) \Gamma\left(v-i p_{n}+\frac{1}{2}\right)} \\
\stackrel{p, p_{n}>>1}{\approx} \frac{\pi e^{-\pi\left(5 p+p_{n}\right) / 2}}{2 p^{2} p_{n}^{2}|\Gamma(i p)|\left|\Gamma\left(i p_{n}\right)\right|}
\end{gathered}
$$

$$
\begin{gathered}
\frac{\mathcal{E}_{\mathbf{L},(n)}^{*} \mathcal{F}_{\mathbf{L}}}{\Gamma\left(v+i p+\frac{1}{2}\right) \Gamma\left(v+i p_{n}+\frac{1}{2}\right)} \\
\stackrel{p, p_{n}>>1}{\approx} \frac{\pi e^{-\pi\left(5 p+p_{n}\right) / 2}}{2 p^{2} p_{n}^{2}|\Gamma(i p)|\left|\Gamma\left(i p_{n}\right)\right|}
\end{gathered}
$$$$
\frac{\mathcal{E}_{\mathbf{L}}^{*} \mathcal{F}_{\mathbf{L},(n)}}{\Gamma\left(v+i p+\frac{1}{2}\right) \Gamma\left(v+i p_{n}+\frac{1}{2}\right)}
$$

$$
\stackrel{p, p_{n}>>1}{\approx} \frac{\pi e^{-\pi\left(p+5 p_{n}\right) / 2}}{2 p^{2} p_{n}^{2}|\Gamma(i p)|\left|\Gamma\left(i p_{n}\right)\right|}
$$

$$
\mathcal{E}_{\mathbf{L},(n)} \mathcal{E}_{\mathbf{L},(m)}^{*}
$$

$$
\overline{\Gamma\left(v-i p_{n}+\frac{1}{2}\right) \Gamma\left(v+i p_{m}+\frac{1}{2}\right)}
$$

$$
p_{n}, p_{m}>>1 \frac{\pi e^{-\pi\left(p_{n}+p_{m}\right) / 2}}{2 p_{n}^{2} p_{m}^{2}\left|\Gamma\left(i p_{m}\right)\right|\left|\Gamma\left(i p_{n}\right)\right|}
$$

$$
\mathcal{F}_{\mathbf{L},(n)} \mathcal{F}_{\mathbf{L},(m)}^{*}
$$

$\overline{\Gamma\left(v-i p_{n}+\frac{1}{2}\right) \Gamma\left(v+i p_{m}+\frac{1}{2}\right)}$

$$
\stackrel{p_{n}, p_{m}>>1}{\approx} \frac{\pi e^{-5 \pi\left(p_{n}+p_{m}\right) / 2}}{2 p_{n}^{2} p_{m}^{2}\left|\Gamma\left(i p_{m}\right)\right|\left|\Gamma\left(i p_{n}\right)\right|}
$$




$$
\begin{aligned}
& \frac{\mathcal{E}_{\mathbf{L},(n)} \mathcal{F}_{\mathbf{L},(m)}^{*}}{\Gamma\left(v-i p_{n}+\frac{1}{2}\right) \Gamma\left(v-i p_{m}+\frac{1}{2}\right)} \\
& p_{n}, \underset{m}{\approx}>>1 \frac{\pi e^{-3 \pi\left(p_{n}+p_{m}\right) / 2}}{2 p_{n}^{2} p_{m}^{2}\left|\Gamma\left(i p_{m}\right)\right|\left|\Gamma\left(i p_{n}\right)\right|} \\
& \mathcal{E}_{\mathbf{L},(n)}^{\sigma *} \mathcal{F}_{\mathbf{L},(m)} \\
& \overline{\Gamma\left(v+i p_{n}+\frac{1}{2}\right) \Gamma\left(v+i p_{m}+\frac{1}{2}\right)} \\
& p_{n}, p_{m}>>1 \frac{\pi e^{-3 \pi\left(p_{n}+p_{m}\right) / 2}}{2 p_{n}^{2} p_{m}^{2}\left|\Gamma\left(i p_{m}\right)\right|\left|\Gamma\left(i p_{n}\right)\right|}
\end{aligned}
$$

Further, we apply Stirling's formula to approximate Gamma functions for large wavenumbers $p, p_{n}>>1$ to simplify the expression for the power spectrum:

$$
\begin{aligned}
\Gamma(i p) \sim & \sqrt{2 \pi}(i p)^{i p-\frac{1}{2}} e^{-i p} \\
& \times\left(1+\frac{1}{12 i p}-\frac{1}{288 p^{2}}+\cdots\right), \\
\Gamma\left(i p_{n}\right) \sim & \sqrt{2 \pi}\left(i p_{n}\right)^{i p_{n}-\frac{1}{2}} e^{-i p_{n}} \\
& \times\left(1+\frac{1}{12 i p_{n}}-\frac{1}{288 p_{n}^{2}}+\cdots\right) .
\end{aligned}
$$

Consequently, we get the following simplified expressio is for large wavenumber $p, p_{n}>>1$ limit in the case of gen. eralised $\alpha$ vacua:

$$
\begin{aligned}
& \frac{\left|\mathcal{E}_{\mathbf{L}}\right|^{2}}{\left|\Gamma\left(v+i p+\frac{1}{2}\right)\right|^{2}} \sim \frac{1}{2 p^{3}\left(1+\frac{1}{82944 p^{4}}\right)} \\
& \left.\frac{\left|\mathcal{F}_{\mathbf{L}}\right|^{2}}{\left|\Gamma\left(v+i p+\frac{1}{2}\right)\right|^{2}} \sim \frac{e^{-4 \pi p}}{2 p^{3}\left(1+\frac{1}{82944} p\right.}\right) \\
& \frac{\mathcal{E}_{\mathbf{L}} \mathcal{F}_{\mathbf{L}}^{*}}{\left(\Gamma\left(v-i p+\frac{1}{2}\right)\right)^{2}} \sim \frac{e^{-}}{2 r^{2(} 1+\frac{1}{\left.8 \cdot \frac{944 p^{4}}{}\right)},},
\end{aligned}
$$

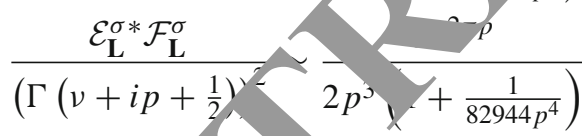

$$
\begin{aligned}
& \mathcal{E}_{\mathbf{L}} \mathcal{E}_{\mathbf{L},(n)}^{*} \\
& \overline{\Gamma(v-j+1) \Gamma\left(v+i p_{n}+\frac{1}{2}\right)} \\
& 2 \frac{1}{3 / 2 p_{n}^{3},-\frac{1}{\sqrt{1+\frac{1}{82944 p^{4}}}} \sqrt{1+\frac{1}{82944 p_{n}^{4}}}} \\
& \frac{\mathcal{F}_{\mathbf{L}} \mathcal{F}_{\mathbf{L},(n)}^{*}}{\Gamma\left(v-i p+\frac{1}{2}\right) \Gamma\left(v+i p_{n}+\frac{1}{2}\right)} \\
& \sim \frac{e^{-2 \pi\left(p+p_{n}\right)}}{2 p^{3 / 2} p_{n}^{3 / 2} \sqrt{1+\frac{1}{82944 p^{4}}} \sqrt{1+\frac{1}{82944 p_{n}^{4}}}} \\
& \mathcal{E}_{\mathbf{L}} \mathcal{F}_{\mathbf{L},(n)}^{*} \\
& \overline{\Gamma\left(v-i p+\frac{1}{2}\right) \Gamma\left(v-i p_{n}+\frac{1}{2}\right)}
\end{aligned}
$$

$$
\sim \frac{e^{-2 \pi p_{n}}}{2 p^{3 / 2} p_{n}^{3 / 2} \sqrt{1+\frac{1}{82944 p^{4}}} \sqrt{1+\frac{1}{82944 p_{n}^{4}}}}
$$$$
\frac{\mathcal{E}_{\mathbf{L},(n)} \mathcal{F}_{\mathbf{L}}^{*}}{\Gamma\left(v-i p+\frac{1}{2}\right) \Gamma\left(v-i p_{n}+\frac{1}{2}\right)}
$$$$
\sim \frac{e^{-2 \pi p}}{2 p^{3 / 2} p_{n}^{3 / 2} \sqrt{1+\frac{1}{82944 p^{4}}} \sqrt{1+\frac{1}{82944 p}}}
$$$$
\mathcal{E}_{\mathbf{L},(n)}^{*} \mathcal{F}_{\mathbf{L}}
$$$$
\overline{\Gamma\left(v+i p+\frac{1}{2}\right) \Gamma\left(v+i p_{n}+\frac{1}{2}\right)}
$$$$
\sim \frac{e^{-2 \pi p}}{2 p^{3 / 2} p_{n}^{3 / 2} \sqrt{1+\frac{1}{829 \cdot 4 p^{4}}}} \frac{}{\sqrt{1+\frac{1}{82} \frac{1}{44 p_{n}^{4}}}}=
$$$$
\frac{\mathcal{E}_{\mathbf{L}}^{*} \mathcal{F}_{\mathbf{L},(n)}}{\left.\Gamma\left(v+i p+\frac{1}{2}\right) \Gamma\left(+i p_{n}+\right)_{\mathcal{L}}\right)}
$$$$
\sim \frac{e}{2 p^{3 / 2} \rho_{n}^{3 / 2} \sqrt{1+\frac{1}{2944 p^{4}}} \sqrt{1+\frac{1}{82944 p_{n}^{4}}}}
$$

$$
\begin{aligned}
& \Gamma\left(v-\frac{1}{2}\right) \Gamma \overline{\left(v+i p_{m}+\frac{1}{2}\right)} \\
& \sim \frac{1}{2 p_{m}^{3} / 2 p_{n}^{3 / 2} \sqrt{1+\frac{1}{82944 p_{m}^{4}}} \sqrt{1+\frac{1}{82944 p_{n}^{4}}}}
\end{aligned}
$$

$$
\begin{gathered}
\frac{\mathcal{F}_{\mathbf{L},(n)} \mathcal{F}_{\mathbf{L},(m)}^{*}}{\left(v-i p_{n}+\frac{1}{2}\right) \Gamma\left(v+i p_{m}+\frac{1}{2}\right)} \\
\sim \frac{e^{-2 \pi\left(p_{n}+p_{m}\right)}}{2 p_{m}^{3 / 2} p_{n}^{3 / 2} \sqrt{1+\frac{1}{82944 p_{m}^{4}}} \sqrt{1+\frac{1}{82944 p_{n}^{4}}}} \\
\frac{\mathcal{E}_{\mathbf{L},(n)} \mathcal{F}_{\mathbf{L},(m)}^{*}}{\Gamma\left(v-i p_{n}+\frac{1}{2}\right) \Gamma\left(v-i p_{m}+\frac{1}{2}\right)} \\
\sim \frac{e^{-\pi\left(p_{n}+p_{m}\right)}}{2 p_{m}^{3 / 2} p_{n}^{3 / 2} \sqrt{1+\frac{1}{82944 p_{m}^{4}}} \sqrt{1+\frac{1}{82944 p_{n}^{4}}}} \\
\frac{\mathcal{E}_{\mathbf{L},(n)}^{*} \mathcal{F}_{\mathbf{L},(m)}}{\Gamma\left(v+i p_{n}+\frac{1}{2}\right) \Gamma\left(v+i p_{m}+\frac{1}{2}\right)} \\
\sim \frac{e^{-\pi\left(p_{n}+p_{m}\right)}}{2 p_{m}^{3 / 2} p_{n}^{3 / 2} \sqrt{1+\frac{1}{82944 p_{m}^{4}}} \sqrt{1+\frac{1}{82944 p_{n}^{4}}}}
\end{gathered}
$$

As a result, in the short wave length approximation the time independent function $\mathcal{Q}(p>>1, \alpha, v)$ for any arbitrary mass parameter $v$ can be expressed for generalised $\alpha$ vacua as:

$$
\begin{aligned}
\mathcal{Q}(p>>1, \alpha, \nu) & =\frac{2^{2(\nu-1)}(\Gamma(\nu))^{2}}{p^{3} \pi} \mathcal{G}(\widetilde{p>>} 1) \\
& =\widetilde{\mathcal{M}(p, v)} \quad \forall \alpha,
\end{aligned}
$$


where we have already defined the function $\mathcal{G}(\widetilde{p>>} 1)$ in the earlier section of the Appendix.

\section{B.2 For small wave number}

Similarly to know the exact wave number dependence of the amplitude of the normalised power spectrum from generalised $\alpha$ vacua in the long wave length approximation we need to know the behaviour of the power spectrum at $p, p_{n}<<1$. After taking this limit it is expected that the power spectrum of axion should match with the result obtained for spatially flat universe. In general for an arbitrary value of the mass parameter $v$, we get the following approximated contributions in the in the long wave length approximation, which are explicitly appearing in the expression for the amplitude of the normalised power spectrum from generalised $\alpha$ vacua:

$$
\begin{aligned}
& \frac{\left|\mathcal{E}_{\mathbf{L}}\right|^{2}}{\left|\Gamma\left(v+i p+\frac{1}{2}\right)\right|^{2}} \stackrel{p<<1}{\approx} \frac{\pi p}{2|\cos \pi v|\left|\Gamma\left(v+\frac{1}{2}\right)\right|^{2}} \\
& \times \frac{\left|1-\gamma_{p}^{(\alpha)} \tilde{m}_{\mathbf{L R}}\right|^{2}}{\left|1-\gamma_{p}^{(\alpha)} \tilde{m}_{\mathbf{L R}}\right|^{2}-\left|\tilde{m}_{\mathbf{R R}}\right|^{2}} \\
& \frac{\left|\mathcal{F}_{\mathbf{L}}\right|^{2}}{\left|\Gamma\left(\nu+i p+\frac{1}{2}\right)\right|^{2}} \stackrel{p<<1}{\approx} \frac{\pi p}{2|\cos \pi \nu|\left|\Gamma\left(\nu+\frac{1}{2}\right)\right|^{2}} \\
& \times \frac{\left|\tilde{m}_{\mathbf{R R}}\right|^{2}}{\left|1-\gamma_{p}^{(\alpha)} \tilde{m}_{\mathbf{L R}}\right|^{2}-\left|\tilde{m}_{\mathbf{R R}}\right|^{2}} \\
& \frac{\mathcal{E}_{\mathbf{L}} \mathcal{F}_{\mathbf{L}}^{*}}{\left(\Gamma\left(\nu-i p+\frac{1}{2}\right)\right)^{2}} \stackrel{p<<1}{\approx} \frac{\pi p}{2|\cos \pi \nu|\left(\Gamma\left(\nu+\frac{1}{2}\right)\right)^{2}} \\
& \times \frac{\left(1-\gamma_{p}^{(\alpha)} \tilde{m}_{\mathbf{L R}}\right) \tilde{m}_{\mathbf{R R}}^{*}}{\left|1-\gamma_{p}^{(\alpha)} \tilde{m}_{\mathbf{L R}}\right|^{2}-\left|\tilde{m}_{\mathbf{R R}}\right|^{2}} \\
& \frac{\mathcal{E}_{\mathbf{L}}^{*} \mathcal{F}_{\mathbf{L}}}{\left(\Gamma\left(\nu+i p+\frac{1}{2}\right)\right)^{2}} \stackrel{p<<1}{\approx} \frac{\pi p}{\left.2|\cos \pi \nu|\left(v^{\prime}+\frac{1}{2}\right)\right)^{-}} \\
& \times \frac{\left(1-\gamma_{p}^{(\alpha)} \tilde{m}_{\mathbf{L R}}\right)^{*} \tilde{m}_{\mathbf{R R}}}{\left|1-\gamma_{p}^{(\alpha)} \tilde{m}_{\mathbf{L R}}\right|^{2}-\left|\tilde{r}_{\mathbf{R}}\right|^{2}} \\
& \frac{\mathcal{E}_{\mathbf{L}} \mathcal{E}_{\mathbf{L},(n)}^{*}}{\Gamma\left(v-i p+\frac{1}{2}\right) \Gamma(\lambda)} \stackrel{p, p_{n}<<1}{\approx} \frac{\pi \sqrt{p p_{n}}}{2|\cos \pi \nu|\left(\Gamma\left(\nu+\frac{1}{2}\right)\right)^{2}}
\end{aligned}
$$

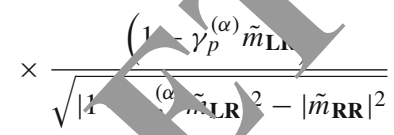

$$
\begin{aligned}
& \frac{\left(1-{ }_{p, n}^{(\alpha)} \tilde{m}_{\mathbf{L R}, n}\right)^{*}}{\left(1-\left.\Gamma_{p, n}^{(\alpha)} \tilde{m}_{\mathbf{L R}, n}\right|^{2}-\left|\tilde{m}_{\mathbf{R R}, n}\right|^{2}\right.} \\
& \frac{\mathcal{F}_{\mathbf{L}} \mathcal{F}_{\mathbf{L},(n)}^{*}}{\Gamma\left(v-i p+\frac{1}{2}\right) \Gamma\left(v+i p_{n}+\frac{1}{2}\right)} \stackrel{p, p_{n}<<1}{\approx} \frac{\pi \sqrt{p p_{n}}}{2|\cos \pi v|\left(\Gamma\left(v+\frac{1}{2}\right)\right)^{2}} \\
& \times \frac{\tilde{m}_{\mathbf{R R}}}{\sqrt{\left|1-\gamma_{p}^{(\alpha)} \tilde{m}_{\mathbf{L R}}\right|^{2}-\left|\tilde{m}_{\mathbf{R R}}\right|^{2}}} \\
& \times \frac{\tilde{m}_{\mathbf{R R}, n}^{*}}{\sqrt{\left|1-\Gamma_{p, n}^{(\alpha)} \tilde{m}_{\mathbf{L R}, n}\right|^{2}-\left|\tilde{m}_{\mathbf{R R}, n}\right|^{2}}}
\end{aligned}
$$

$$
\begin{aligned}
& \frac{\mathcal{E}_{\mathbf{L}} \mathcal{F}_{\mathbf{L},(n)}^{*}}{\Gamma\left(v-i p+\frac{1}{2}\right) \Gamma\left(v-i p_{n}+\frac{1}{2}\right)} \stackrel{p, p_{n}<<1}{\approx} \frac{\pi \sqrt{p p_{n}}}{2|\cos \pi v|\left(\Gamma\left(v+\frac{1}{2}\right)\right)^{2}} \\
& \times \frac{\left(1-\gamma_{p}^{(\alpha)} \tilde{m}_{\mathbf{L R}}\right)}{\sqrt{\left|1-\gamma_{p}^{(\alpha)} \tilde{m}_{\mathbf{L R}}\right|^{2}-\left|\tilde{m}_{\mathbf{R R}}\right|^{2}}} \\
& \times \frac{\tilde{m}_{\mathbf{R R}, n}^{*}}{\sqrt{\left|1-\Gamma_{p, n}^{(\alpha)} \tilde{m}_{\mathbf{L R}, n}\right|^{2}-\left|\tilde{m}_{\mathbf{R R}, n}\right|^{2}}}
\end{aligned}
$$

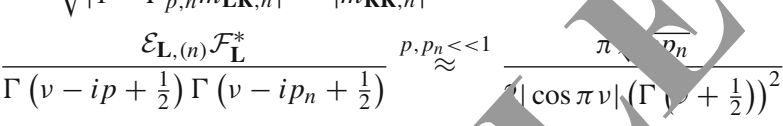

$$
\begin{aligned}
& \times \frac{\left(1-\Gamma_{p, n}^{(\alpha)} \tilde{m}_{\mathbf{L R}, n}\right)}{\sqrt{\left|1-\Gamma_{p, n}^{(\alpha)} \tilde{m}_{\mathbf{L R}, n}\right|^{2}-\mid \tilde{m}_{\mathbf{R R},}}}= \\
& \times \frac{\tilde{m}_{\mathbf{R R}}^{*}}{\sqrt{\left|1-\gamma_{p}^{(\alpha)} \tilde{m}_{\mathbf{L R}}\right|^{2}\left\langle\tilde{m}_{\mathbf{R R}}\right|}} \\
& \mathcal{E}_{\mathbf{L},(n)}^{*} \mathcal{F}_{\mathbf{T}} \underset{p, p_{n}<<1}{\approx} \pi \sqrt{p p_{n}} \\
& \overline{\Gamma\left(v+i p+\frac{1}{2}\right) \Gamma\left(v+i p_{n}\right.} \overline{2|\cos \pi v|\left(\Gamma\left(v+\frac{1}{2}\right)\right)^{2}} \\
& \times \frac{\Gamma}{\sqrt{\sqrt{1-\Gamma_{p, n}^{(\alpha)} m_{\mathbf{2}},\left.n\right|^{2}-\left|\tilde{m}_{\mathbf{R R}, n}\right|^{2}}}} \\
& \times \frac{{ }_{\sqrt{\mid 1}}-\left.\overbrace{p}^{(\alpha)} \tilde{m}_{\mathbf{L R}}\right|^{2}-\left|\tilde{m}_{\mathbf{R R}}\right|^{2}}{\tilde{l}_{\mathbf{R R}}}
\end{aligned}
$$$$
\frac{\mathcal{E}_{\mathbf{L}}^{*} \mathcal{F}_{\mathbf{L},(n)}}{\left.1+i p+\frac{1}{2}\right) \Gamma\left(v+i p_{n}+\frac{1}{2}\right)} \stackrel{p, p_{n}<<1}{\approx} \frac{\pi \sqrt{p p_{n}}}{2|\cos \pi v|\left(\Gamma\left(v+\frac{1}{2}\right)\right)^{2}}
$$$$
\begin{aligned}
& \times \frac{\left(1-\gamma_{p}^{(\alpha)} \tilde{m}_{\mathbf{L R}}\right)^{*}}{\sqrt{\left|1-\gamma_{p}^{(\alpha)} \tilde{m}_{\mathbf{L R}}\right|^{2}-\left|\tilde{m}_{\mathbf{R R}}\right|^{2}}} \\
& \times \frac{\tilde{m}_{\mathbf{R R}, n}}{\sqrt{\left|1-\Gamma_{p, n}^{(\alpha)} \tilde{m}_{\mathbf{L R}, n}\right|^{2}-\left|\tilde{m}_{\mathbf{R R}, n}\right|^{2}}}
\end{aligned}
$$$$
\frac{\mathcal{E}_{\mathbf{L},(n)} \mathcal{E}_{\mathbf{L},(m)}^{*}}{\Gamma\left(v-i p_{n}+\frac{1}{2}\right) \Gamma\left(v+i p_{m}+\frac{1}{2}\right)} \stackrel{p_{n}, p_{m}<<1}{\approx} \frac{\pi \sqrt{p_{n} p_{m}}}{2|\cos \pi v|\left(\Gamma\left(v+\frac{1}{2}\right)\right)^{2}}
$$$$
\times \frac{\left(1-\Gamma_{p, n}^{(\alpha)} \tilde{m}_{\mathbf{L R}, n}\right)}{\sqrt{\left|1-\Gamma_{p, n}^{(\alpha)} \tilde{m}_{\mathbf{L R}, n}\right|^{2}-\left|\tilde{m}_{\mathbf{R R}, n}\right|^{2}}}
$$$$
\times \frac{\left(1-\Gamma_{p, m}^{(\alpha)} \tilde{m}_{\mathbf{L R}, m}\right)^{*}}{\sqrt{\left|1-\Gamma_{p, m}^{(\alpha)} \tilde{m}_{\mathbf{L R}, m}\right|^{2}-\left|\tilde{m}_{\mathbf{R R}, m}\right|^{2}}}
$$

$$
\begin{aligned}
& \frac{\mathcal{F}_{\mathbf{L},(n)} \mathcal{F}_{\mathbf{L},(m)}^{*}}{\Gamma\left(v-i p_{n}+\frac{1}{2}\right) \Gamma\left(v+i p_{m}+\frac{1}{2}\right)} \stackrel{p_{n}, p_{m}<<1}{\approx} \frac{\pi \sqrt{p_{n} p_{m}}}{2|\cos \pi v|\left(\Gamma\left(v+\frac{1}{2}\right)\right)^{2}} \\
& \times \frac{\tilde{m}_{\mathbf{R R}, n}}{\sqrt{\left|1-\Gamma_{p, n}^{(\alpha)} \tilde{m}_{\mathbf{L R}, n}\right|^{2}-\left|\tilde{m}_{\mathbf{R R}, n}\right|^{2}}}
\end{aligned}
$$$$
\times \frac{\tilde{m}_{\mathbf{R R}, m}^{*}}{\sqrt{\left|1-\Gamma_{p, m}^{(\alpha)} \tilde{m}_{\mathbf{L R}, m}\right|^{2}-\left|\tilde{m}_{\mathbf{R R}, m}\right|^{2}}}
$$$$
\frac{\mathcal{E}_{\mathbf{L},(n)} \mathcal{F}_{\mathbf{L},(m)}^{*}}{\Gamma\left(v-i p_{n}+\frac{1}{2}\right) \Gamma\left(v-i p_{m}+\frac{1}{2}\right)} \stackrel{p_{n}, p_{m}<<1}{\approx} \frac{\pi \sqrt{p_{n} p_{m}}}{2|\cos \pi v|\left(\Gamma\left(v+\frac{1}{2}\right)\right)^{2}}
$$$$
\times \frac{\left(1-\Gamma_{p, n}^{(\alpha)} \tilde{m}_{\mathbf{L R}, n}\right)}{\sqrt{\left|1-\Gamma_{p, n}^{(\alpha)} \tilde{m}_{\mathbf{L R}, n}\right|^{2}-\left|\tilde{m}_{\mathbf{R R}, n}\right|^{2}}}
$$ 


$$
\begin{aligned}
& \times \frac{\tilde{m}_{\mathbf{R R}, m}^{*}}{\sqrt{\left|1-\Gamma_{p, m}^{(\alpha)} \tilde{m}_{\mathbf{L R}, m}\right|^{2}-\left|\tilde{m}_{\mathbf{R R}, m}\right|^{2}}} \\
& \frac{\mathcal{E}_{\mathbf{L}, n)}^{*} \mathcal{F}_{\mathbf{L},(m)}}{\Gamma\left(v+i p_{n}+\frac{1}{2}\right) \Gamma\left(v+i p_{m}+\frac{1}{2}\right)} \stackrel{p_{n}, p_{m}<<1}{\sim} \frac{\pi \sqrt{p_{n} p_{m}}}{2|\cos \pi \nu|\left(\Gamma\left(v+\frac{1}{2}\right)\right)^{2}} \\
& \times \frac{\left(1-\Gamma_{p, n}^{(\alpha)} \tilde{m}_{\mathbf{L R}, n}\right)^{*}}{\sqrt{\left|1-\Gamma_{p, n}^{(\alpha)} \tilde{m}_{\mathbf{L R}, n}\right|^{2}-\left|\tilde{m}_{\mathbf{R R}, n}\right|^{2}}} \\
& \times \frac{\tilde{m}_{\mathbf{R R}, m}}{\sqrt{\left|1-\Gamma_{p, m}^{(\alpha)} \tilde{m}_{\mathbf{L R}}\right|^{2}-\left|\tilde{m}_{\mathbf{R R}, m}\right|^{2}}}
\end{aligned}
$$

where all the entries of the right hand side of the above expressions for $p, p_{n}<<1$ are explicitly computed earlier in this paper.

As a result, the time independent function $\mathcal{Q}(p \widetilde{<<1}, \alpha, v)$ for the mass parameter $v \neq q / 2$ (where $q$ is any half integer) can be expressed for generalised $\alpha$ vacua as:

$$
\begin{aligned}
\mathcal{Q} & (p \widetilde{<<1}, \alpha, v) \\
& =\frac{2^{2(\nu-1)}(\Gamma(v))^{2}}{p^{3} \pi} \mathcal{G}(\widetilde{p<<} 1) \quad \forall \alpha,
\end{aligned}
$$

where the function $\mathcal{G}(\widetilde{p<<})$ is defined for $v \neq 3 / 2$ as:

$$
\begin{aligned}
& \mathcal{G}(\widetilde{p<<1)} \\
& =\frac{\pi p}{2|\cos \pi \nu|\left|\Gamma\left(v+\frac{1}{2}\right)\right|^{2}} \frac{\left|1-\gamma_{p}^{(\alpha)} \tilde{m}_{\mathbf{L R}}\right|^{2}}{\left|1-\gamma_{p}^{(\alpha)} \tilde{m}_{\mathbf{L R}}\right|^{2}-\left|\tilde{m}_{\mathbf{R R}}\right|^{2}} \\
& \times\left\{1+\frac{\left|\tilde{m}_{\mathbf{R R}}\right|^{2}+\left(1-\gamma_{p}^{(\alpha)} \tilde{m}_{\mathbf{L R}}\right)^{*} \tilde{m}_{\mathbf{R R}}+\left(1-\gamma\left(\alpha \tilde{m}_{\mathbf{L}}\right.\right.}{\left|1-\gamma_{p}^{(\alpha)} \tilde{m}_{\mathbf{L R}}\right|^{2}}\right\} \\
& \left.+\sum_{n=0}^{\infty} \sqrt{\frac{p_{n}}{p} \frac{\left|1-\gamma_{p}^{(\alpha)} \tilde{m}_{\mathbf{L R}}\right|^{2}-\left|\tilde{m}_{\mathbf{R R}}\right|^{2}}{\left|1-\Gamma_{p, n}^{(\alpha)} \tilde{m}_{\mathbf{L R}, n}\right|^{2}-\left|\tilde{m}_{\mathbf{R}, n}\right|^{2}}} \mid 1-1\right)\left.\right|_{\left.\mathbf{R}\right|^{2}} \\
& {\left[\left(1-\gamma_{p}^{(\alpha)} \tilde{m}_{\mathbf{L R}}\right)\left(1-\Gamma_{p, n}^{(\alpha)} \tilde{m}_{\mathbf{L R}, n}\right)^{*}+m_{\mathbf{K}} \gamma_{, n}\right.} \\
& \left.+\left(1-\gamma_{p}^{(\alpha)} \tilde{m}_{\mathbf{L R}}\right) \tilde{m}_{\mathbf{R R}, n}^{*} \Gamma_{p, n}^{(\alpha)} \tilde{m}_{\mathbf{1}} \mathbf{2}, n\right) \tilde{m}_{\mathbf{R R}}^{*} \\
& \left.+\left(1-\gamma_{p}^{(\alpha)} \tilde{m}_{\mathbf{L R}}\right)^{*} \tilde{m}_{\mathbf{R} \mathbf{R}, n}\left(1-p_{p, n} \tilde{m}_{\mathbf{L R}, n}\right)^{*} \tilde{m}_{\mathbf{R R}}\right] \\
& +\sum_{n=0}^{\infty} \sum_{m=0}^{\infty} \\
& \sqrt{\frac{p_{n, m}}{p^{2}}\left(\left.\Gamma_{n, h}^{(\alpha)} \tilde{m}_{\mathbf{L R}, n}\right|^{2}-\left|\tilde{m}_{\mathbf{R R}, n}\right|^{2}\right)\left(\left|1-\Gamma_{p, m}^{(\alpha)} \tilde{m}_{\mathbf{L R}, m}\right|^{2}-\left|\tilde{m}_{\mathbf{R R}, m}\right|^{2}\right)} \\
& \left(1-\gamma_{p, m}^{(\alpha)} \tilde{m}_{\mathbf{L R}, m}\right)^{*}+\tilde{m}_{\mathbf{R R}, n} \tilde{m}_{\mathbf{R R}, m}^{*} \\
& +\left(1-\Gamma_{p, n}^{(\alpha)} \tilde{m}_{\mathbf{L R}, n}\right) \tilde{m}_{\mathbf{R R}, m}^{*}+\left(1-\Gamma_{p, n}^{(\alpha)} \tilde{m}_{\mathbf{L R}, n}\right) \tilde{m}_{\mathbf{R R}, m}^{*} \\
& \left.\left.+\left(1-\Gamma_{p, n}^{(\alpha)} \tilde{m}_{\mathbf{L R}, \mathbf{n}}\right)^{*} \tilde{m}_{\mathbf{R R}, m}+\left(1-\Gamma_{p, n}^{(\alpha)} \tilde{m}_{\mathbf{L R}, n}\right)^{*} \tilde{m}_{\mathbf{R R}, m}\right]\right\}
\end{aligned}
$$

On the other hand, if we set $v=q / 2$ (including the massless case for $v=3 / 2$ ) in the previous expressions obtained for general $v$ then due to the presence of the overall factor $1 /|\cos \pi \nu|$ the final expression for the power spectrum in small wave number limit diverges. This is very obvious from the obtained expressions but one can be able to avoid such unwanted divergent contributions very easily. To serve this purpose let us rewrite all the expressions for $p, p_{n}<<1$ with $v=q / 2$ that we have mentioned earlier.

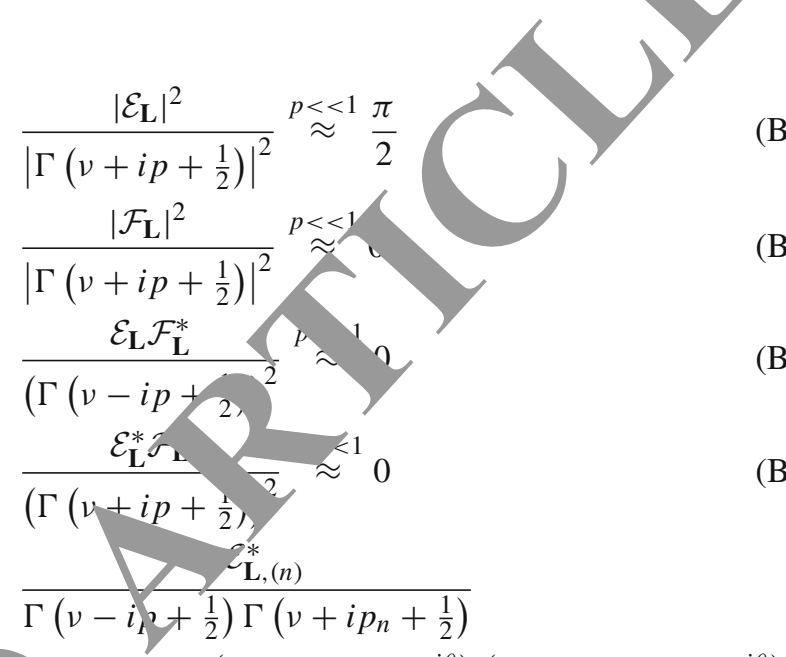

$\stackrel{p, p_{n}<<1}{\approx} \frac{\pi}{2} \frac{\left(1 \pm \pi p e^{-p \pi} e^{i \theta}\right)}{\left|1 \pm \pi p e^{-p \pi} e^{i \theta}\right|} \frac{\left(1 \pm \pi p_{n} e^{-p_{n} \pi} e^{-i \theta}\right)}{\mid 1 \pm \pi p_{n} e^{-p_{n} \pi} e^{i \theta \mid}}$

$$
\begin{aligned}
& \frac{\mathcal{F}_{\mathbf{L}} \mathcal{F}_{\mathbf{L},(n)}^{*}}{\Gamma\left(v-i p+\frac{1}{2}\right) \Gamma\left(v+i p_{n}+\frac{1}{2}\right)} \stackrel{p, p_{n}<<1}{\approx} 0 \\
& \frac{\mathcal{E}_{\mathbf{L}} \mathcal{F}_{\mathbf{L},(n)}^{*}}{\Gamma\left(v-i p+\frac{1}{2}\right) \Gamma\left(v-i p_{n}+\frac{1}{2}\right)} \stackrel{p, p_{n}<<1}{\approx} 0 \\
& \frac{\mathcal{E}_{\mathbf{L},(n)} \mathcal{F}_{\mathbf{L}}^{*}}{\Gamma\left(v-i p+\frac{1}{2}\right) \Gamma\left(v-i p_{n}+\frac{1}{2}\right)} \stackrel{p, p_{n}<<1}{\approx} 0 \\
& \frac{\mathcal{E}_{\mathbf{L},(n)}^{*} \mathcal{F}_{\mathbf{L}}}{\Gamma\left(v+i p+\frac{1}{2}\right) \Gamma\left(v+i p_{n}+\frac{1}{2}\right)} \stackrel{p, p_{n}<<1}{\approx} 0 \\
& \frac{\mathcal{E}_{\mathbf{L}}^{*} \mathcal{F}_{\mathbf{L},(n)}}{\Gamma\left(v+i p+\frac{1}{2}\right) \Gamma\left(v+i p_{n}+\frac{1}{2}\right)} \stackrel{p, p_{n}<<1}{\approx} 0 \\
& \frac{\mathcal{E}_{\mathbf{L},(n)} \mathcal{E}_{\mathbf{L},(m)}^{*}}{\Gamma\left(v-i p_{n}+\frac{1}{2}\right) \Gamma\left(v+i p_{m}+\frac{1}{2}\right)} \\
& \stackrel{p_{n}, p_{m}}{\approx}<<1 \frac{\pi}{2} \frac{\left(1 \pm \pi p_{n} e^{-p_{n} \pi} e^{i \theta}\right)}{\left|1 \pm \pi p_{n} e^{-p_{n} \pi} e^{i \theta}\right|} \frac{\left(1 \pm \pi p_{m} e^{-p_{m} \pi} e^{i \theta}\right)}{\mid 1 \pm \pi p_{m} e^{-p_{m} \pi} e^{i \theta \mid}}
\end{aligned}
$$

$\frac{\mathcal{F}_{\mathbf{L},(n)} \mathcal{F}_{\mathbf{L},(m)}^{*}}{\Gamma\left(v-i p_{n}+\frac{1}{2}\right) \Gamma\left(v+i p_{m}+\frac{1}{2}\right)} \stackrel{p_{n}, p_{m}<<1}{\approx} 0$

$\frac{\mathcal{E}_{\mathbf{L},(n)} \mathcal{F}_{\mathbf{L},(m)}^{*}}{\Gamma\left(v-i p_{n}+\frac{1}{2}\right) \Gamma\left(v-i p_{m}+\frac{1}{2}\right)} \stackrel{p_{n}, p_{m}<<1}{\approx} 0$

$\frac{\mathcal{E}_{\mathbf{L},(n)}^{\sigma *} \mathcal{F}_{\mathbf{L},(m)}}{\Gamma\left(v+i p_{n}+\frac{1}{2}\right) \Gamma\left(v+i p_{m}+\frac{1}{2}\right)} \stackrel{p_{n}, p_{m}<<1}{\approx} 0$ 
Also for the massless case $(v=3 / 2)$ the time independent function $\mathcal{Q}(p<\widetilde{1, \alpha, v}=3 / 2)$ can be further simplified as:

$$
\begin{aligned}
\mathcal{Q} & (p<\widetilde{1, \alpha, \nu}=3 / 2) \\
& =\frac{\mathcal{G}(p<\widetilde{<1, \nu}=3 / 2)}{2 p^{3}} \forall \alpha,
\end{aligned}
$$

where the function $\mathcal{G}(\widetilde{p<<})$ is defined for $v=3 / 2$ as:

$$
\begin{aligned}
\mathcal{G}(p & <\widetilde{<1, v}=3 / 2) \\
= & \frac{\pi}{2}\left\{1+\frac{\left(1 \pm e^{i \theta} \pi p e^{-p \pi}\right)}{\left|1 \pm e^{i \theta} \pi p e^{-p \pi}\right|} \sum_{n=0}^{\infty} \frac{\left(1 \pm e^{-i \theta} \pi p_{n} e^{-p_{n} \pi}\right)}{\left|1 \pm e^{i \theta} \pi p_{n} e^{-p_{n} \pi}\right|}\right. \\
& \left.+\sum_{n=0}^{\infty} \sum_{m=0}^{\infty} \sqrt{\frac{\left(1 \pm e^{i \theta} \pi p_{n} e^{-p_{n} \pi}\right)}{\left|1 \pm e^{i \theta} \pi p_{n} e^{-p_{n} \pi}\right|} \frac{\left(1 \pm e^{-i \theta} \pi p_{m} e^{-p_{m} \pi}\right)}{\left|1 \pm e^{i \theta} \pi p_{m} e^{-p_{m} \pi}\right|}}\right\}
\end{aligned}
$$

\section{Quantum correction to the power spectrum in NES formalism}

At the superhorizon time scales $\left(t_{\mathbf{L}}>>1\right)$ of region $\mathbf{L}$ amplitude of the NES power spectrum can be expressed as:

$$
\left|\tilde{\phi}^{\mathbf{L}}\right|^{2} \underset{t_{\mathbf{L}}>>1}{\longrightarrow} \widetilde{\mathcal{K}}(\widetilde{p, \alpha, \nu})\left(\cosh t_{\mathbf{L}}\right)^{2 v-1}
$$

where the time independent function $\mathcal{K}(0,0, v)$ generalised $\alpha$ vacua is defined as:

$$
\begin{aligned}
& \widetilde{\mathcal{K}(p, \alpha, v)} \\
& =\frac{2^{2 v-1}(\Gamma(v))^{2}}{\pi} \times\left[\frac{\mid \Gamma\left(1-T^{2}\right.}{2 p\left|\Gamma\left(v+i \mid{ }^{2}+\frac{1}{2}\right)\right|^{2}}\right. \\
& +\sum_{n=0}^{\infty} \\
& \times \frac{\mid \Gamma(1-i p)}{\infty}\left(\frac{\overline{\bar{p}}}{\infty} \mid \frac{\left.1+i p_{n}\right)_{1}+|\Gamma(1+i p)|\left|\Gamma\left(1-i p_{n}\right)\right|}{\left.-i p+\frac{1}{2}\right) \Gamma\left(v+i p_{n}+\frac{1}{2}\right)}\right.
\end{aligned}
$$

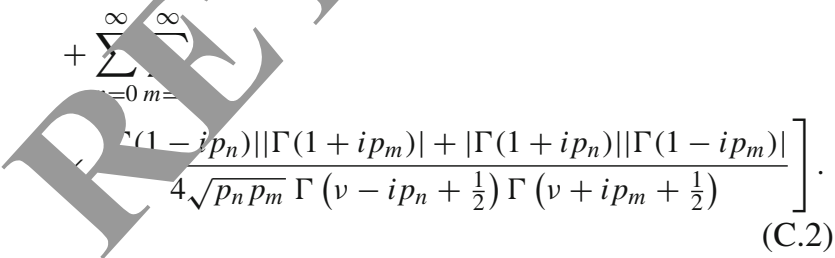

\section{C.1 For large wave number}

Further, to know the exact wave number dependence of the amplitude of the normalised power spectrum from generalised $\alpha$ vacua we need to know the behaviour of the power spectrum at very short wavelengths $\left(p, p_{n}>>1\right)$. After taking this limit it is expected that the power spectrum of axion in the non entangled case should match with the result obtained for spatially flat universe. In general for an arbitrary value of the mass parameter $v$, we get the following approximated contributions in the short wavelength limit $\left(p, p_{n}>>1\right)$, which are explicitly appearing in the expressic nt tor the amplitude of the normalised power spectrum f $\mathrm{g}$ erc ised $\alpha$ vacua:

$$
\begin{aligned}
& \frac{|\Gamma(1+i p)|^{2}}{2 p\left|\Gamma\left(v+i p+\frac{1}{2}\right)\right|^{2}} \stackrel{p>>1}{\approx} \frac{1}{2 p^{3}}, \\
& \left.\frac{|\Gamma(1-i p)|\left|\Gamma\left(1+i p_{n}\right)\right|}{4 \sqrt{p p_{n}} \Gamma\left(v-i p^{2}\right.}+\frac{\mid \mathrm{I}}{1}+i p\right) \mid \frac{\left|\Gamma\left(1-i p_{n}\right)\right|}{\left.i p_{n}+\frac{1}{2}\right)} \\
& p, \stackrel{p, p_{n}>>1}{\approx} \frac{1}{2\left(p p_{n}\right)^{\frac{3}{2}}} \\
& \frac{\mid \Gamma\left(1-i p_{n}\right)}{4 \sqrt{\rho} n_{n}} \frac{\left.+i p_{m}\right)_{1}+\left|\Gamma\left(1+i p_{n}\right)\right|\left|\Gamma\left(1-i p_{m}\right)\right|}{\left.i p_{n}+\frac{1}{2}\right) \Gamma\left(v+i p_{m}+\frac{1}{2}\right)} \\
& p_{n}, p_{m}>>1-\frac{Y}{\left(p_{n} p_{m}\right)^{\frac{3}{2}}} \text {. } \\
& +\frac{\mid i p)\left|\Gamma\left(1-i p_{n}\right)\right|}{\left.i p_{n}+\frac{1}{2}\right)}
\end{aligned}
$$

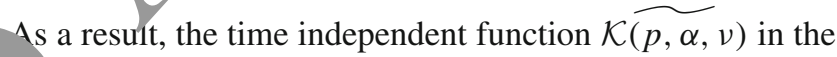
rt wave length limit for any arbitrary mass parameter $v$ ca 1 be expressed for generalised $\alpha$ vacua as:

$\mathcal{K}(p \widetilde{>}, \alpha, \nu)=\frac{2^{2(\nu-1)}(\Gamma(v))^{2}}{p^{3} \pi} \mathcal{U}(\widetilde{p>>} 1) \quad \forall \alpha$,

where the function $\mathcal{U}(\widetilde{p>>} 1)$ is defined as:

$$
\begin{aligned}
& \mathcal{U}(\widetilde{p>>} 1) \\
& \quad=\left[1+\sum_{n=0}^{\infty}\left(\frac{p}{p_{n}}\right)^{\frac{3}{2}}+\sum_{n=0}^{\infty} \sum_{m=0}^{\infty} \frac{p^{3}}{\left(p_{n} p_{m}\right)^{\frac{3}{2}}}\right] .
\end{aligned}
$$

Here for very large wave number $p, p_{n}>>1$ one can write, $\widetilde{\mathcal{U}(p)} \sim 1+\cdots$, where all $\cdots$ are small correction terms. This also implies to the nice fact that for large wave number limit for any values of the parameter $\alpha$ the time independent function $\mathcal{U}(p, \alpha, v)$ computed for generalised $\alpha$ vacua is exactly matches with the result obtained for Bunch Davies vacua in the earlier section at the leading order in $\widetilde{\mathcal{M}(p, v)}$.

Also for the massless case $(v=3 / 2)$ the time independent function $\mathcal{K}(p, \widetilde{\alpha, v=3 / 2})$ in the short wave length limit can be further simplified as:

$\mathcal{K}(p>>\widetilde{1, \alpha, v}=3 / 2)=\frac{\mathcal{U}(\widetilde{p>>} 1)}{2 p^{3}} \quad \forall \alpha$ 


\section{C.2 For small wave number}

Similarly to see the behaviour of the power spectrum in the long wavelength region in the super horizon time scale $\left(t_{\mathbf{L}}>>1\right)$ we take the limit $p<<1$ and further expand the expression for the power spectrum in $p$. In general for an arbitrary value of the mass parameter $v$, we get the following approximated contributions in the long wavelength limit $\left(p, p_{n}<<1\right)$, which are explicitly appearing in the expression for the amplitude of the normalised power spectrum from generalised $\alpha$ vacua:

$$
\begin{aligned}
& \frac{|\Gamma(1+i p)|^{2}}{2 p\left|\Gamma\left(v+i p+\frac{1}{2}\right)\right|^{2}} \stackrel{p<<1}{\approx} \frac{1}{2 p\left|\Gamma\left(v+\frac{1}{2}\right)\right|^{2}}, \\
& \frac{|\Gamma(1-i p)|\left|\Gamma\left(1+i p_{n}\right)\right|+|\Gamma(1+i p)|\left|\Gamma\left(1-i p_{n}\right)\right|}{4 \sqrt{p p_{n}} \Gamma\left(v-i p+\frac{1}{2}\right) \Gamma\left(v+i p_{n}+\frac{1}{2}\right)} \\
& \stackrel{p, p_{n}<<1}{\approx} \frac{1}{2 \sqrt{p p_{n}}\left(\Gamma\left(v+\frac{1}{2}\right)\right)^{2}} \\
& \frac{\left|\Gamma\left(1-i p_{n}\right)\right|\left|\Gamma\left(1+i p_{m}\right)\right|+\left|\Gamma\left(1+i p_{n}\right)\right|\left|\Gamma\left(1-i p_{m}\right)\right|}{4 \sqrt{p_{n} p_{m}} \Gamma\left(v-i p_{n}+\frac{1}{2}\right) \Gamma\left(v+i p_{m}+\frac{1}{2}\right)} \\
& \stackrel{1}{p_{n}, p_{m}<<1} \frac{1}{2 \sqrt{p_{n} p_{m}}\left(\Gamma\left(v+\frac{1}{2}\right)\right)^{2}} .
\end{aligned}
$$

As a result, in the long wave length limit the time independe it function $\mathcal{K} \widetilde{(p, \alpha, v)}$ for any arbitrary mass parameter $v$ ca be expressed for generalised $\alpha$ vacua as:

$$
\mathcal{K}(p \widetilde{<<1}, \alpha, v)=\frac{2^{2(\nu-1)}(\Gamma(v))^{2}}{p \pi} \mathcal{U}(\widetilde{p<}, \forall \alpha,
$$

where the function $\mathcal{U}(\widetilde{p<<} 1)$ is de $d$ in the long wave length limit as:

$$
\begin{aligned}
\mathcal{U}(\widetilde{p<<1}) \\
\left.\quad=\left[1+\left(\frac{\mid \Gamma\left(v+\frac{1}{2}\right)}{\Gamma\left(\nu+\frac{1}{2}\right)}\right) \sum_{=0}^{\infty} \sqrt{\frac{p}{p_{n}}}+\sum_{n=0}^{\infty} \sum_{m=0}^{\infty} \frac{p}{\sqrt{p_{n} p_{m}}}\right\}\right] .
\end{aligned}
$$

Also for 1 assless case $(v=3 / 2)$ the time independent function $\mathcal{K}, \alpha_{0}=3 / 2$ ) can be further simplified as:

$\mathcal{K}(p) \mathcal{1}, \alpha, v=3 / 2)=\frac{\mathcal{U}(\widetilde{p<<})}{2 p} \quad \forall \alpha$.

\section{References}

1. R. Horodecki, P. Horodecki, M. Horodecki, K. Horodecki, Quantum entanglement. Rev. Mod. Phys. 81, 865 (2009). arXiv:quant-ph/0702225
2. E. Martin-Martinez, N.C. Menicucci, Cosmological quantum entanglement. Class. Quant. Grav. 29, 224003 (2012). arXiv: 1204.4918 [gr-qc]

3. Y. Nambu, Entanglement of quantum fluctuations in the inflationary universe. Phys. Rev. D 78, 044023 (2008). arXiv:0805.1471 [gr-qc]

4. J.S. Bell, On the Einstein-Podolsky-Rosen paradox. Physics 1, 195 (1964)

5. S.R. Coleman, F. De Luccia, Gravitational effec s on and of vacuum decay. Phys. Rev. D 21, 3305 (1980)

6. J. Garriga, S. Kanno, M. Sasaki, J. Soda, A. Vin in Observer dependence of bubble nucleation and chwinger pa. production. JCAP 1212, 006 (2012). arXiv:1208. $\quad$ Thep-tl

7. J. Garriga, S. Kanno, T. Tanaka, est frai f bubble nucleation. JCAP 1306, 034 (2013). arXiy 1304.6681 [h $\rho$-th]

8. M.B. Fröb, J. Garriga, S. Kal o, M. Sasaki, J. Soda, T. Tanaka, A. Vilenkin, Schwinger $f$ fect Sitt r space. JCAP 1404, 009 (2014). arXiv:1401.4137 -th]

9. W. Fischler, P.H. N ayen, J.h draza, W. Tangarife, Holographic Schwinger effect Sitter si ace. Phys. Rev. D 91(8), 086015 (2015). arXiv:1.11.1, Thep-th]

10. N. Lafloren Quantur ntanglement in condensed matter systems. Ph Re . 646, 1 (2016). arXiv:1512.03388 [cond-mat.strel]

11. S. Ryu, T. vanagi, Holographic derivation of entanglement en from ,dS/CFT. Phys. Rev. Lett. 96, 181602 (2006). arX van 2603001

12. T. Ta ayanagi, Entanglement entropy from a holographic viewpoint. Cass. Quant. Grav. 29, 153001 (2012). arXiv:1204.2450 [gr-qc]

S. Ryu, T. Takayanagi, Holographic derivation of entanglement entropy from AdS/CFT. Phys. Rev. Lett. 96, 181602 (2006). arXiv:hep-th/0603001

14. S. Ryu, T. Takayanagi, Aspects of holographic entanglement entropy. JHEP 0608, 045 (2006). arXiv:hep-th/0605073

15. T. Nishioka, S. Ryu, T. Takayanagi, Holographic entanglement entropy: an overview. J. Phys. A 42, 504008 (2009). arXiv:0905.0932 [hep-th]

16. M. Rangamani, T. Takayanagi, Holographic entanglement entropy. Lect. Notes Phys. 931, (2017). arXiv:1609.01287 [hep-th]

17. V.E. Hubeny, M. Rangamani, T. Takayanagi, A Covariant holographic entanglement entropy proposal. JHEP 0707, 062 (2007). arXiv:0705.0016 [hep-th]

18. J. Maldacena, G.L. Pimentel, Entanglement entropy in de Sitter space. JHEP 1302, 038 (2013). arXiv: 1210.7244 [hep-th]

19. S. Kanno, J. Murugan, J.P. Shock, J. Soda, Entanglement entropy of $\alpha$-vacua in de Sitter space. JHEP 1407, 072 (2014). arXiv: 1404.6815 [hep-th]

20. B. Allen, Vacuum states in de Sitter space. Phys. Rev. D 32, 3136 (1985)

21. K. Goldstein, D.A. Lowe, A note on alpha vacua and interacting field theory in de Sitter space. Nucl. Phys. B 669, 325 (2003). arXiv:hep-th/0302050

22. J. de Boer, V. Jejjala, D. Minic, Alpha-states in de Sitter space. Phys. Rev. D 71, 044013 (2005). arXiv:hep-th/0406217

23. R. Brunetti, K. Fredenhagen, S. Hollands, A Remark on alpha vacua for quantum field theories on de Sitter space. JHEP 0505, 063 (2005). arXiv:hep-th/0503022

24. S. Choudhury, S. Panda, Entangled de Sitter from stringy axionic Bell pair I: an analysis using Bunch-Davies vacuum. Eur. Phys. J. C 78(1), 52 (2018). arXiv:1708.02265 [hep-th]

25. S. Choudhury, S. Panda, Quantum entanglement in de Sitter space from stringy axion: an analysis using $\alpha$ vacua. arXiv:1712.08299 [hep-th]

26. J. Maldacena, A model with cosmological Bell inequalities. Fortsch. Phys. 64, 10 (2016). arXiv:1508.01082 [hep-th] 
27. S. Choudhury, S. Panda, R. Singh, Bell violation in the Sky. Eur. Phys. J. C 77(2), 60 (2017). arXiv:1607.00237 [hep-th]

28. S. Choudhury, S. Panda, R. Singh, Bell violation in primordial cosmology. Universe 3(1), 13 (2017). arXiv:1612.09445 [hep-th]

29. S. Kanno, J. Soda, Infinite violation of Bell inequalities in inflation. arXiv:1705.06199 [hep-th]

30. S. Panda, Y. Sumitomo, S.P. Trivedi, Axions as quintessence in string theory. Phys. Rev. D 83, 083506 (2011). arXiv:1011.5877 [hep-th]

31. L. McAllister, E. Silverstein, A. Westphal, Gravity waves and linear inflation from axion monodromy. Phys. Rev. D 82, 046003 (2010). arXiv:0808.0706 [hep-th]

32. E. Silverstein, A. Westphal, Monodromy in the CMB: gravity waves and string inflation. Phys. Rev. D 78, 106003 (2008). arXiv:0803.3085 [hep-th]

33. L. McAllister, E. Silverstein, A. Westphal, T. Wrase, The powers of monodromy. JHEP 1409, 123 (2014). arXiv:1405.3652 [hep-th]

34. S. Kanno, Impact of quantum entanglement on spectrum of cosmological fluctuations. JCAP 1407, 029 (2014). arXiv: 1405.7793 [hep-th]

35. S. Choudhury, S. Panda, COSMOS- $e$-GTachyon from string theory. Eur. Phys. J. C 76(5), 278 (2016). arXiv:1511.05734 [hep-th]

36. S. Choudhury, COSMOS- $e^{\prime}$-soft Higgsotic attractors. Eur. Phys. J. C 77(7), 469 (2017). arXiv:1703.01750 [hep-th]

37. S. Choudhury, S. Pal, Primordial non-Gaussian features from DBI Galileon inflation. Eur. Phys. J. C 75(6), 241 (2015). arXiv: 1210.4478 [hep-th]

38. S. Choudhury, S. Pal, DBI Galileon inflation in background SUGRA. Nucl. Phys. B 874, 85 (2013). arXiv:1208.4433 [hep-th]

39. S. Choudhury, S. Pal, Fourth level MSSM inflation from new flat directions. JCAP 1204, 018 (2012). arXiv:1111.3441 [hep-ph]

40. S. Choudhury, S. Pal, Brane inflation in background supergraviı Phys. Rev. D 85, 043529 (2012). arXiv:1102.4206 [hep-tt/_

41. S. Choudhury, Can Effective Field Theory of inflat $n$ gener ate large tensor-to-scalar ratio within Randall-Sundru ingle braneworld? Nucl. Phys. B 894, 29 (2015). arXiv · 406.761 th]

42. S. Choudhury, B.K. Pal, B. Basu, P. Bandvopadhy Quantum gravity effect in torsion driven inflation and CP violat $n$. JHEP 1510, 194 (2015). arXiv:1409.6036 [he -th]

43. S. Choudhury, Reconstructing inflation paradig n within effective field theory framework. Phys. Da Iniv 11, 16 (2016). arXiv:1508.00269 [astro-ph.CO]

44. S. Choudhury, A. Mazumdar, A a a bound on tensor-toscalar ratio and the scale of inflation vucl. Phys. B 882, 386 (2014). arXiv:1306.4496 [hep- $\mathrm{ph}]$

45. S. Choudhury, A. M. nd ordial blackholes and gravitational waves for infle n-point model of inflation. Phys. Lett. B 733, 270 (20 arXiv: 1 . $/ .5119$ [astro-ph.CO]

46. S. Choudhu , A. zumdar, Reconstructing inflationary potential from BIC 2 2 and ru. 8 of tensor modes. arXiv:1403.5549 [hepth]

47. S. Ca th .y, A Mazumdar, E. Pukartas, Constraining $\mathcal{N}=$ 1 superg. ty $j_{A}$ flationary framework with non-minimal Kähler op tors. J LP 1404, 077 (2014). arXiv:1402.1227 [hep-th]

4 S. r sury, Constraining $\mathcal{N}=1$ supergravity inflation with -minımal Kaehler operators using $\delta \mathrm{N}$ formalism. JHEP 1404, 10. 2014). [arXiv:1402.1251 [hep-th]]

49. S. Choudhury, A. Mazumdar, S. Pal, Low \& High scale MSSM inflation, gravitational waves and constraints from Planck. JCAP 1307, 041 (2013). arXiv:1305.6398 [hep-ph]
50. J. Maharana, S. Mukherji, S. Panda, Notes on axion, inflation and graceful exit in stringy cosmology. Mod. Phys. Lett. A 12, 447 (1997). arXiv:hep-th/9701115

51. A. Mazumdar, S. Panda, A. Perez-Lorenzana, Assisted inflation via tachyon condensation. Nucl. Phys. B 614, 101 (2001). arXiv:hep-ph/0107058

52. D. Choudhury, D. Ghoshal, D.P. Jatkar, S. Panda, Hybrid inflation and brane-anti-brane system. JCAP 03/ 009 (2003). arXiv:hep-th/0305104

53. D. Choudhury, D. Ghoshal, D.P. Jatkar, S. Pat onthe cosmological relevance of the tachyon. Phys. Lett. B 221 (2002). arXiv:hep-th/0204204

54. P. Chingangbam, S. Panda, A. Desham $\mathrm{v}$ va, Nor minimally coupled tachyonic inflation in warper outing oround. JHEP 0502, 052 (2005). arXiv:hep-th/0411 10

55. A. Deshamukhya, S. Panda, rm tachy nnic inflation in warped background. Int. J. Mod. P'ys. $\quad 2092$ (2009). arXiv:0901.0471 [hep-th]

56. P. Vargas Moniz, S. Tanda, J. d. Higher order corrections to Heterotic M-theory in. ion. Class Quant. Grav. 26, 245003 (2009). arXiv:0907.07 ^ [ast h.CO]

57. A. Ali, A hamukh, S. Panda, M. Sami, Inflation with improver D3-b ane potential and the fine tunings associated with the mode.... J.C 71, 1672 (2011). arXiv:1010.1407 [hepth]

58. A. Pattacharjye, A. Deshamukhya, S. Panda, A note on low eneigy ive theory of chromo-natural inflation in the light of B1 2EP2 results. Mod. Phys. Lett. A 30(11), 1550040 (2015). arXiv: 406.5858 [astro-ph.CO]

S. Panda, M. Sami, S. Tsujikawa, Inflation and dark energy arising from geometrical tachyons. Phys. Rev. D 73, 023515 (2006). arXiv:hep-th/0510112

S. Panda, M. Sami, S. Tsujikawa, J. Ward, Inflation from D3-brane motion in the background of D5-branes. Phys. Rev. D 73, 083512 (2006). arXiv:hep-th/0601037

61. S. Panda, M. Sami, S. Tsujikawa, Prospects of inflation in delicate D-brane cosmology. Phys. Rev. D 76, 103512 (2007). arXiv:0707.2848 [hep-th]

62. D. Baumann, TASI lectures on Inflation (2009). arXiv:0907.5424 [hep-th]

63. D. Baumann, A. Dymarsky, I.R. Klebanov, L. McAllister, Towards an explicit model of D-brane inflation. JCAP 0801, 024 (2008). arXiv:0706.0360 [hep-th]

64. D. Baumann, L. McAllister, Advances in inflation in string theory. Ann. Rev. Nucl. Part. Sci. 59, 67 (2009). arXiv:0901.0265 [hep-th]

65. V. Assassi, D. Baumann, D. Green, Symmetries and loops in inflation. JHEP 1302, 151 (2013). arXiv:1210.7792 [hep-th]

66. D. Baumann, L. McAllister, inflation and string theory. arXiv:1404.2601 [hep-th]

67. D. Baumann, A. Dymarsky, S. Kachru, I.R. Klebanov, L. McAllister, Holographic systematics of D-brane inflation. JHEP 0903, 093 (2009). arXiv:0808.2811 [hep-th]

68. H.V. Peiris, D. Baumann, B. Friedman, A. Cooray, Phenomenology of D-brane inflation with general speed of sound. Phys. Rev. D 76, 103517 (2007). arXiv:0706.1240 [astro-ph]

69. P. Svrcek, E. Witten, Axions in string theory. JHEP 0606, 051 (2006). arXiv:hep-th/0605206 